


\section{Anticipatory Freight Scheduling in Synchromodal Transport}

Arturo E. Pérez Rivera 
Graduation committee:

Chairman and Secretary: Prof. dr. T.A.J. Toonen

Promotor:

Prof. dr. J. van Hillegersberg

Co-promotor:

Dr. ir. M.R.K. Mes

Members:

Prof. dr. R.J. Boucherie

Universiteit Twente

Prof. dr. M.E. Iacob

Universiteit Twente

Prof. dr. D.C. Mattfeld

Technische Universität

Braunschweig

Prof. dr. ir. L.A. Tavasszy

Technische Universiteit

Delft

Prof. dr. S. Voß

Universität Hamburg

Prof. dr. W.H.M. Zijm

Universiteit Twente

Prof. dr. R. Zuidwijk

Erasmus Universiteit

Rotterdam

Ph.D. thesis, University of Twente, Digital Society Institute,

Department of Industrial Engineering and Business Information Systems

This thesis is part of the Ph.D. thesis series of the Beta Research School for Operations Management and Logistics (onderzoeksschool-beta.nl) in which the following universities cooperate: Eindhoven University of Technology, Maastricht University, University of Twente, VU Amsterdam, Wageningen University and Research, and KU Leuven. The research contained in this thesis has been partially funded by the Dutch Institute for Advanced Logistics (DINALOG), under the project SynchromodalIT.

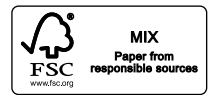

Printed by Ipskamp Printing, Enschede, The Netherlands.

Cover photograph credits: iStock.com/Opla. Aerial view of the APM container terminal and different transport modes on the Maasvlakte in Rotterdam, The Netherlands.

Cover design: Martijn R.K. Mes and Arturo E. Pérez Rivera.

(C) A.E. Pérez Rivera, 2018, Enschede, The Netherlands.

All rights reserved. No part of this publication may be reproduced without the prior written permission of the author.

ISBN: $\quad 978-90-365-4571-6$

ISSN: $\quad$ 2589-7721 (DSI Ph.D. Thesis Series No. 18-003)

DOI: $\quad 10.3990 / 1.9789036545716$ 


\title{
ANTICIPATORY FREIGHT SCHEDULING IN SYNCHROMODAL TRANSPORT
}

\section{DISSERTATION}

\author{
to obtain \\ the degree of doctor at the University of Twente, \\ on the authority of the rector magnificus, \\ Prof. dr. T.T.M. Palstra, \\ on account of the decision of the graduation committee, \\ to be publicly defended \\ on Friday $29^{\text {th }}$ of June 2018 at 14:45 hrs.
}

by

\section{Arturo Eduardo Pérez Rivera}

born on the $2^{\text {nd }}$ of March 1988

in San Pedro Sula, Honduras. 
This doctoral dissertation is approved by the Promotor,

Prof. dr. J. van Hillegersberg

and the Co-promotor,

Dr. ir. M.R.K. Mes 
A mi madre, Roselia Arelí, y a mi padre, Justo Edelmín. 


\section{Contents}

Preamble

Contents $\quad$ ix

1 Introduction 1

1.1 Synchromodality . . . . . . . . . . . . . . . . . 2

1.2 Theoretical Motivation . . . . . . . . . . . . . . . . . . 8

1.3 Research Design . . . . . . . . . . . . . . . . . . . . . . . . . . . . . . . . 10

1.4 Thesis Outline .......................... 14

2 Long-haul Round-trip Transport: Solution Design 19

2.1 Introduction . . . . . . . . . . . . . . . . . . . 19

2.2 Literature Review . . . . . . . . . . . . . . . . . . . . . . . . . . 21

2.3 Mathematical Model . . . . . . . . . . . . . . . . . . . 23

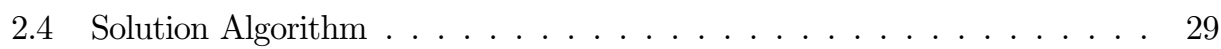

2.5 Experimental Setup . . . . . . . . . . . . . . . . . . . . . . . . . . . . . 33

2.6 Numerical Results . . . . . . . . . . . . . . . . . . . . 36

2.7 Discussion . . . . . . . . . . . . . . . . . . . . . . . 41

2.8 Conclusions . . . . . . . . . . . . . . . . . . . . . . . . . . . . . . . . . . .

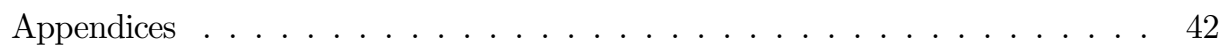

3 Long-haul Round-trip Transport: Solution Analysis 47

3.1 Introduction . . . . . . . . . . . . . . . . . . . . . . 47

3.2 Experimental Setup . . . . . . . . . . . . . . . . . . . . . . 48

3.3 Numerical Results . . . . . . . . . . . . . . . . . . . . . . 51

3.4 Single-trip Interlude . . . . . . . . . . . . . . . . . . . . . . . 52

3.5 Discussion . . . . . . . . . . . . . . . . . . . . 55

3.6 Conclusions . . . . . . . . . . . . . . . . . 57 
4 Long-haul Multi-transfer Transport $\quad 59$

4.1 Introduction . . . . . . . . . . . . . . . . . . . 59

4.2 Problem Description . . . . . . . . . . . . . . . . . . 61

4.3 Literature Review . . . . . . . . . . . . . . . . . . . . . . . 62

4.4 Mathematical Model . . . . . . . . . . . . . . . . . . . . 64

4.5 Solution Algorithm . . . . . . . . . . . . . . . . . . 71

4.6 Numerical Experiments . . . . . . . . . . . . . . . . . . . . . 82

4.7 Discussion . . . . . . . . . . . . . . . . . . . . . . 93

4.8 Conclusions . . . . . . . . . . . . . . . . . . . 94

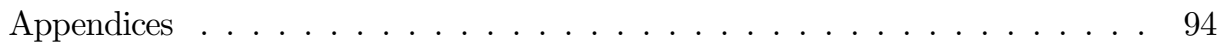

5 Multi-terminal Drayage Transport 101

5.1 Introduction . . . . . . . . . . . . . . . . . . . . . . . 101

5.2 Literature Review . . . . . . . . . . . . . . . . . . . . . . . . . . . . . . . . . . . . . . . . . . . .

5.3 Problem Description . . . . . . . . . . . . . . . . . . . . 103

5.4 Mathematical Model . . . . . . . . . . . . . . . . . . . . 105

5.5 Solution Algorithm . . . . . . . . . . . . . . . . . . 110

5.6 Numerical Experiments . . . . . . . . . . . . . . . . . . . 112

5.7 Discussion . . . . . . . . . . . . . . . . . . . 116

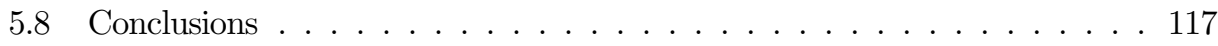

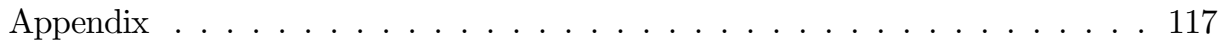

6 Integrated Long-haul and Drayage Transport 121

6.1 Introduction . . . . . . . . . . . . . . . . . . . 121

6.2 Literature Review . . . . . . . . . . . . . . . . . . . . . . . 123

6.3 Problem Description and Formulation . . . . . . . . . . . . . . . . . . 124

6.4 Solution Approach . . . . . . . . . . . . . . . . . . . 135

6.5 Numerical Experiments . . . . . . . . . . . . . . . . . . . . . 139

6.6 Discussion . . . . . . . . . . . . . . . . . . . . . . . . . . . . . . . . . . . . . . . . . . . . . . .

6.7 Conclusions . . . . . . . . . . . . . . . . . . . . . . . . . . . . . . . . . . . . . . . . . . . . .

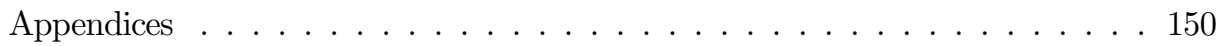

7 Raising Awareness About Anticipatory Scheduling 157

7.1 Introduction . . . . . . . . . . . . . . . . . 157

7.2 Background . . . . . . . . . . . . . . . . . . 158

7.3 Game Design . . . . . . . . . . . . . . . . . . . . . . . . . . . . . . . . . . . . . . . . . . . . . . . . .

7.4 Game Test . . . . . . . . . . . . . . . . . . . . . 173

7.5 Conclusions . . . . . . . . . . . . . . . . . . 180

8 Conclusions and Prospects 183

8.1 Conclusions . . . . . . . . . . . . . . . . . . 183

8.2 Outlook for Research and Implementation . . . . . . . . . . . . . . . . 190

8.3 Closing Thoughts . . . . . . . . . . . . . . . . . 194 
$\begin{array}{ll}\text { Bibliography } & 195\end{array}$

$\begin{array}{ll}\text { Acronyms } & 207\end{array}$

$\begin{array}{lr}\text { Summary } & 209\end{array}$

$\begin{array}{lr}\text { Samenvatting } & 213\end{array}$

$\begin{array}{lr}\text { Resumen } & 217\end{array}$

$\begin{array}{ll}\text { Scientific Output } & 221\end{array}$

$\begin{array}{ll}\text { Acknowledgements / Agradecimientos } & 225\end{array}$

$\begin{array}{lr}\text { About the Author } & 229\end{array}$ 


\section{Introduction}

The expansion of freight transport in the European Union (EU) has been proportional to its economical development but also to its increased congestion and pollution. From 1995 to 2014, the amount of tonne-kilometers of freight transport in the EU increased around 24\% and the Gross Domestic Product (GDP) increased around 35\% [29]. During the same time, the emissions of greenhouse gases resulting from freight transport increased around $11 \%$ [29]. The largest portion of this increment was due to road transport of freight. The road transport mode, which was estimated in 2017 [31] to yield 139.8 grams of $\mathrm{CO}_{2}$ per tonne-kilometer, increased its amount of tonne-kilometers with 33.9\% [29]. In contrast, rail freight transport, which was estimated to be the most environmental friendly transport mode in 2017 [31] with 15.6 grams of $\mathrm{CO}_{2}$ per tonne-kilometer , only grew $5.8 \%$ during the same period of time [29]. Although rail transport has more restrictions than road transport (e.g., infrastructure, governmental regulations, etc.), using it more can mitigate the negative environmental impact of freight transport by road. Furthermore, using rail and other high-capacity transport modes can increase the sustainability of freight transport and thus support the related economical development.

The need for efficient freight transport with minimum environmental impact has been recognized by various stakeholders in the logistics industry. These stakeholders have set goals and initiated actions to achieve such sustainable freight transport. For instance, the European Commission set the goal of a $30 \%$ shift of road freight to other modes such as rail or barge, and a fully-functional EU-wide intermodal network, by the year 2030 [28]. From 2011, when the goal was introduced, to 2014, the share of the road mode in freight transport has decreased from $75.1 \%$ to $74.9 \%$ [33]. Although this reduction is small, a foreseeable and larger reduction is expected due to the nine European Rail Freight Corridors that became operational in the end of 2015 [30]. In a similar way to the EU, the governmental Top Sector Logistics in The Netherlands (Topsector Logistiek in Dutch) set goals to increase the overall load factor of transport equipment by $20 \%$ from its level in 2011 [102], and to reduce 35 million kilometers of road freight transport by 2020 [104]. So far, initiatives by the Top Sector 
Logistics such as 'Lean \& Green Synchromodal' have achieved a reduction of 1.5 million kilometers of road transport [105]. Such initiatives involve governmental organizations, port authorities, Logistic Service Providers (LSPs), shippers, academic knowledge centers, and other stakeholders, to produce innovations that make freight transport efficient and sustainable. One of these initiatives, which we study in this thesis, is synchromodality.

Synchromodality is a management paradigm for freight transport based on a multi-modal network that involves the dynamic choice of transport mode for freight orders, at different parts of the orders' route, to bring them from their origin to their destination within the agreed limits [104]. The choice is dynamic in the sense that an order's transport plan (i.e., route and combination of modes) is not fixed at the beginning of transport but constructed at various decision moments during the transport process, considering the latest information about the transport network, and optimizing network-wide performance indicators such as costs and $\mathrm{CO}_{2}$ emissions. Due to the flexibility in execution of freight transport in synchromodality, more and better consolidation opportunities arise over the network and over time [109]. However, to identify and seize these opportunities, changes to the planning and control of traditional multi-modal freight transport are required. In this thesis, we focus on the changes required for the operational planning and control of synchromodal transport, although we note that changes are required at all management levels. Particularly, we study changes in the choice of mode and route for freight orders at different points of the transport process, considering dynamic decisions that use the latest information about the transport network, and with the objective of optimizing performance over the network and over time.

In this introductory chapter, we explain our approach to study the changes to traditional multi-modal transport planning that synchromodal transport requires. The chapter is organized as follows. In Section 1.1, we further describe synchromodality, its requirements and its implications. In addition, we define the scope of our study with respect to planning synchromodal transport. In Section 1.2, we briefly describe how the characteristics of synchromodality give rise to new research opportunities. In Section 1.3, we define our research objectives and research questions. Furthermore, we define the steps towards achieving our research objective and answering our research questions. Finally, in Section 1.4, we outline the structure of the thesis and present an overview of its contents.

\subsection{Synchromodality}

The intensification of freight moves and its negative environmental impact have driven stakeholders in the logistics industry to re-think the ways in which freight transport is organized. A new way of organizing freight transport that has been proposed in The Netherlands is synchromodality [108]. According to the Dutch Topsector Logistiek [103], synchromodality is multi-modal transport where the LSPs synchronously employ the available modes of transport and intermodal terminals, to bring freights to their destination at the agreed conditions such as time-windows, costs, emissions, etc. In synchromodal transport, freights are booked with less restrictions on the transport mode and ideally "mode-free". Although synchromodal transport is closely related to intermodal and co-modal transport, it differs in three aspects: (i) it is not limited to the same loading unit/container, (ii) it is not 
limited to a single group of stakeholders, and (iii) it emphasizes the flexibility to change routes for freight at any time [92]. The flexibility in transport mode and route results in opportunities to improve efficiency and sustainability. According to van Riessen, Negenborn, and Dekker [108], the possible outcome of this increased flexibility is twofold. First, the increased flexibility should result in more bundling possibilities and larger use of the available rail and inland shipping capacity. The increased utilization of rail and inland shipping will in turn reduce costs and emissions. Second, the increased flexibility should result in increased reliability, e.g., better on-time performance, since uncertainties and disturbances are handled easier.

Although the positive outcomes of synchromodality are enabled by the lowered restrictions on the mode and route of transport, there are various other requirements to realize them. The successful implementation of synchromodal transport has infrastructure, policy, and technology requirements [87]. The infrastructure requirements refer to a multi-modal transport network with sufficient amount of services or transport modes, and sufficient connectivity between them (e.g., transfer terminals and speed of transfers). Although these requirements also hold for other forms of multi-modal transport, the emphasis in synchromodal transport is on the possibility to change transport plans (e.g., mode and route) multiple times within a freight's transport process. The policy requirements refer to industry guidelines and governmental regulations. For example, restrictions of customers for a particular mode of transport, or route, must be low or ideally non-existing. Furthermore, LSPs must have a network-wide perspective about performance and about operations rather than the common unilateral view. Governmental regulations should promote, or guide, customers and LSPs towards such behavior. The technology requirements refer to the interoperability among operations in the various parts of the transport process and the overview, and control, of network-wide circumstances and performance. Interoperability deals with the swift and reliable flow of information among operations that enables multiple transport plan changes. The overview and control of network-wide circumstances and performance involves not only setting a larger objective, but also including the dynamic and stochastic nature of some of the network parameters into the planning of a freight's transport.

In this thesis, we consider that infrastructure and policy requirements for synchromodality are satisfied, and that interoperability among operations in the transport process is present. We limit our research to the last requirement: the overview and control of network-wide circumstances and performance. Specifically, we study the day-to-day planning of freight transport in a synchromodal network. Since this type of planning involves the assignment of freights to modes, routes, terminals, and services in the transport network through time, we refer to it as scheduling in the remainder of this thesis. Even though the scheduling of freight in a synchromodal network takes place at an operational level, its relation to time means that both immediate and future consequences on the performance of the entire network have to be considered, as we explain next.

Immediate consequences of a scheduling decision are those that are known directly after making the decision and with complete certainty. For example, consider an LSP transporting freights from the hinterland to various terminals in a deep-sea port, on a daily basis, using one barge each day. After assigning (and loading) some freights to today's barge service, the scheduler knows the immediate consequences of the decision made: terminals where 
the barge must stop to unload those freights and the costs for their handling and transport. Future consequences of a scheduling decision, on the other hand, are those that are not known directly after making the decision, or that are not known with complete certainty. Following the previous example, consider the freights that were not assigned to today's barge service. The scheduler can assign some them, for instance, to the barge departing in two days from today. Future consequences are the potential terminal visits (and costs) from the barge departing in two days. These future consequences are deemed as potential since the scheduler has the possibility of changing plans tomorrow, perhaps when new freight might arrive that present a better consolidation opportunity for that day. Since some future consequences of scheduling decisions are subject to variability in demand (e.g., number of freights, destination of freights, etc.) and variability in supply (e.g., capacity of a barge due to water levels, traveling times, etc.), their results can only be estimated with some degree of uncertainty.

For achieving the positive outcomes of synchromodal transport, the scheduling of freight must consider both the immediate and future consequences of decisions on the network-wide performance. We refer to decisions that simultaneously take into account immediate consequences and look-ahead for future consequences as anticipatory scheduling decisions. In the following section, we exemplify the need of anticipatory scheduling to achieve the positive outcomes of synchromodal transport and the implications that anticipatory scheduling decisions might have over a multi-modal transport process.

\subsubsection{Anticipatory Scheduling in Synchromodality}

The objective of anticipatory scheduling in synchromodality is to optimize the immediate and future performance of multi-modal transport processes. This multi-period view on performance requires that schedulers analyze the evolution of the network when making decisions about those processes. To exemplify such a view and analysis, consider the following example of a container transport process. Every day, a high-capacity mode transports containers from a single origin to far-away destinations within a region, as shown in Figure 1.1. Think, for example, of a barge transporting containers from the hinterland to various terminals in a deep-sea port. In addition, there is an alternative transport mode (e.g., truck) that can take a container at a higher price than the high-capacity mode. Each day, new containers with different destinations arrive. Containers are not known before they arrive, but there is probabilistic information on their arrival. All containers must be transported to their destination at the lowest costs possible.

Consider a time horizon of three days. In Figure 1.2 and Figure 1.3, we exemplify two ways of scheduling: (i) "myopic" and (ii) anticipatory, respectively. In these figures, the containers scheduled for transport each day are indicated with a check-mark. The myopic schedule optimizes the immediate performance, e.g., it maximizes the utilization of the high-capacity mode and minimizes the route length to deliver all containers each day. The anticipatory schedule, on the contrary, postpones the transport of some containers to minimize the route to deliver the chosen containers, with a possibly lower utilization than the myopic schedule for the same day. Although simplistic, the example shows different optimization views and their consequences. 


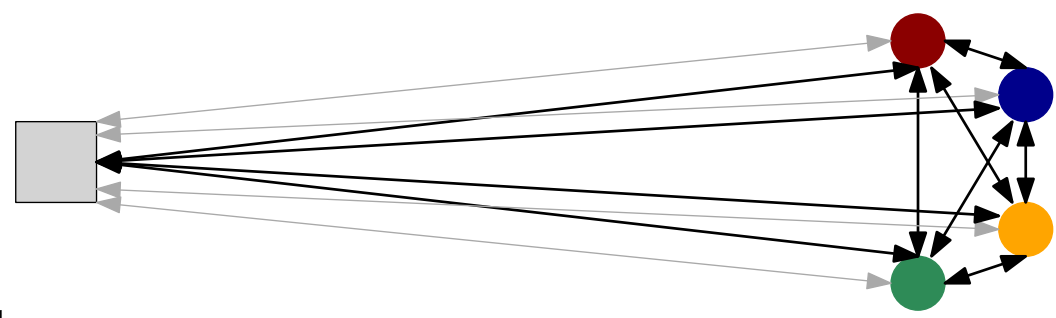

Legend:

Origin $\bigcirc \bigcirc$ Destinations $\longrightarrow$ Alternative mode $\longleftrightarrow$ High-capacity mode

Figure 1.1: Example of a transport process in synchromodality

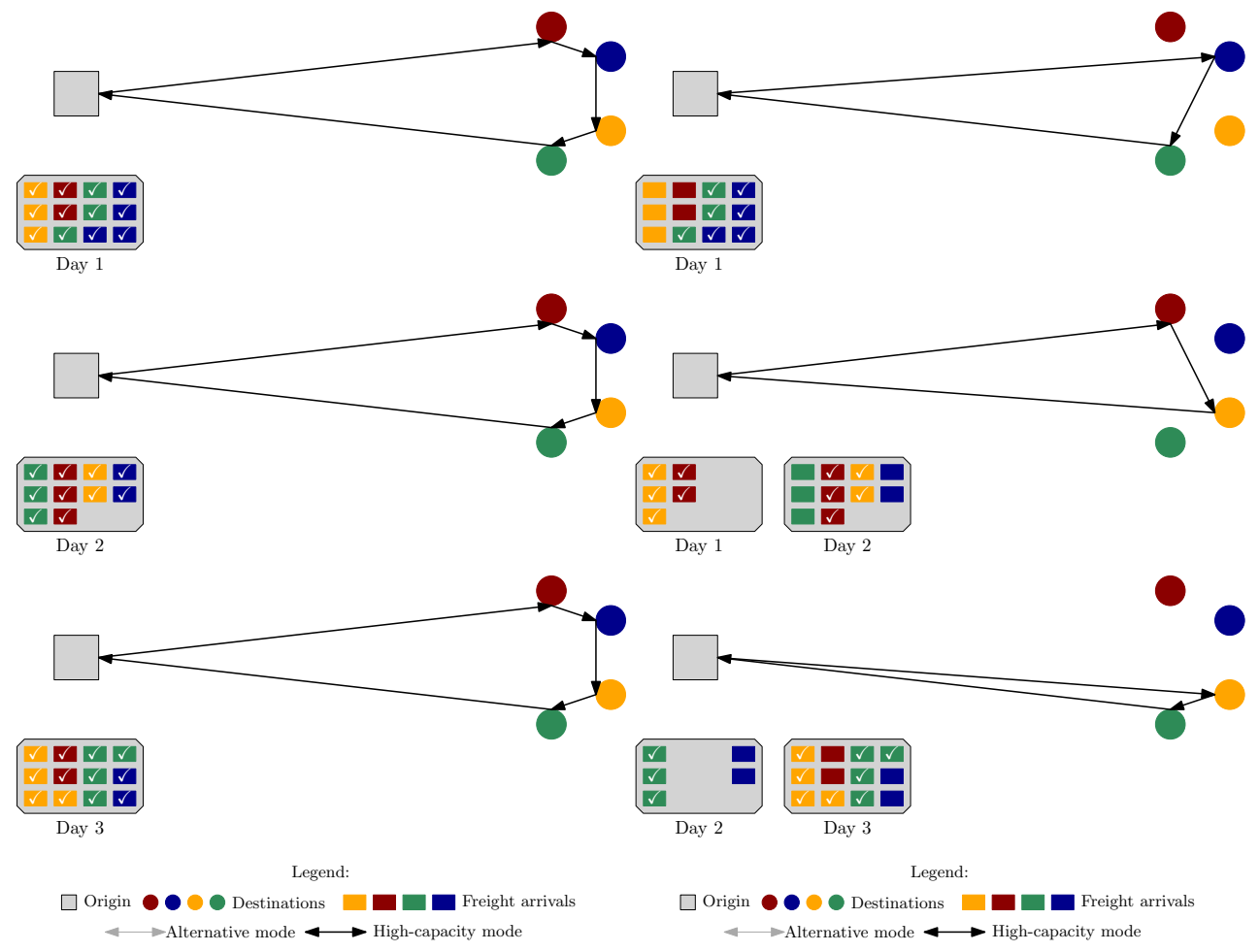

Figure 1.2: Example of a myopic schedule

Figure 1.3: Example of an anticipatory schedule

In the anticipatory schedule of the previous example, postponing the transport of containers also postpones the costs. These postponed costs, however, may be lower than the the same day costs of a myopic schedule if better consolidation opportunities arise (e.g., new containers going to the same terminals). Naturally, forecasting which containers will 
arrive and estimating which postponements may result in lower costs in the future is not straightforward. For this reason, anticipatory scheduling brings more challenges than myopic scheduling. Besides deciding which freights to deliver and how to route the high-capacity mode each day, there is the challenge of how to evaluate the expected situation of tomorrow (since freights are not known beforehand) and how to balance the performance obtained today with the one expected tomorrow.

Even though the example above is a simplification of a real problem, it reveals that the aspects to consider for anticipatory scheduling decisions are diverse and intricate. The relations among services in the network, freight demand, and decisions, become even more difficult to analyze when considering more aspects in synchromodality, such as transfer terminals, time-windows, service schedules, traveling times, etc. Nevertheless, and as the example shows, anticipating on future performance is favorable when there is information about the stochastic nature of these relations (e.g., arrival of containers). Thanks to the advancements in information technologies, such as increased use of sensors in transport modes and infrastructure, open data sources, and predictive analytics, the measurement, study, and control of such relations is increasingly attainable. This information trend has opened new research opportunities in the area of scheduling multi-modal freight transport [92, 56], such as the concept of synchromodality, and the overall management of logistic processes. In the following section, we briefly elaborate on how synchromodality and anticipatory scheduling relate to this trend, from a governmental and from an industrial example.

\subsubsection{The Future of Logistic Processes}

Synchromodality involves a network-wide view and integrated control of transport processes enabled by information technologies. Such characteristics are not exclusive to the synchromodality concept. In fact, many concepts about the future of logistic processes, such as Hyperconnected City Logistics [20], Logistics 4.0 [44], and the Physical Internet [64], involve the aforementioned characteristics. Although these concepts differ in some details, they all have in common the physical and information integration of multiple logistics services, with the goal of improving their efficiency and sustainability. Therefore, the anticipatory scheduling aspects described in the previous section are applicable, to some extent, to these concepts about the future of logistics processes. In this section, we describe some of these concepts, based on government and industry perspectives, and their relation to anticipatory scheduling in synchromodality.

The first concept is the so-called Physical Internet (PI). In analogy to the digital Internet where information is transferred over a vast network, in the PI there is an open and fully connected network of logistics services (e.g., assemble, transport, store, etc.) and logistics service users (e.g., shippers, carriers, manufacturers), where physical objects are seamlessly transferred among the connected parties through standardized encapsulation and information protocols [64]. The EU Technology Platform ALICE [32] recognizes that synchromodal services are a necessary step to realize the PI in 2050, as seen in Figure 1.4. In the synchromodal services envisioned the PI, anticipatory scheduling relates to the control protocols for the transport and transfer of physical objects through the integrated network. Such a scheduling approach can be used for a dynamic consolidation of freight based on 
the latest information about demand and supply capacity of transport processes.

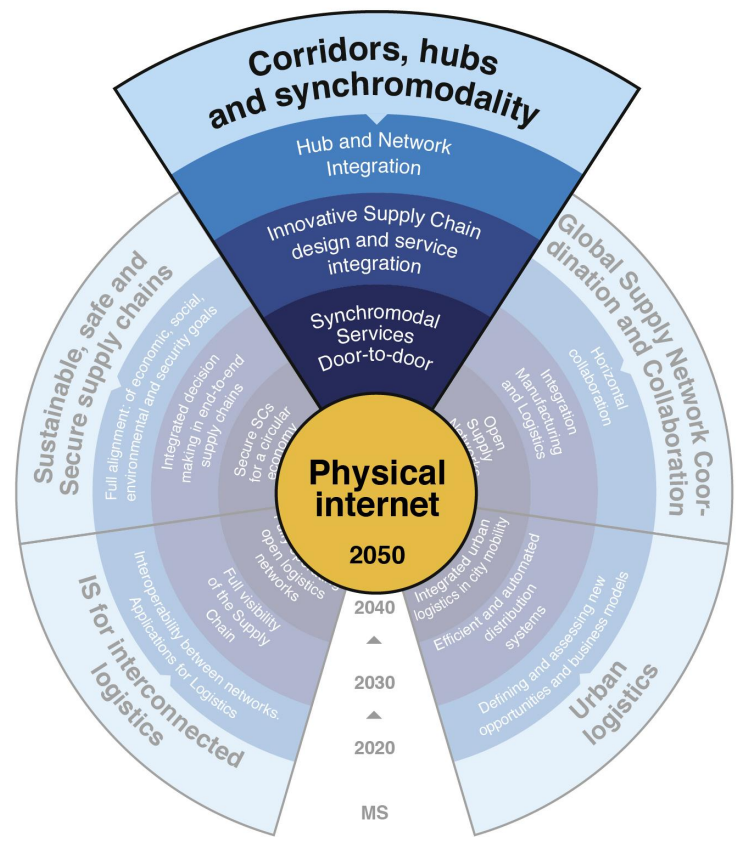

Figure 1.4: The Physical Internet according to the EU Platform ALICE [32]

In industry, the logistics service provider DHL recognizes various trends where anticipatory scheduling of synchromodal transport is relevant. In its Trend Radar 2016 [24], the three trends with the largest connection to anticipatory scheduling are: (i) anticipatory logistics, (ii) logistics marketplaces, and (iii) supergrid logistics. These three trends are pushed by society and businesses, and are foreseen to have a high impact on the way logistics industry is organized, as seen in Figure 1.5. In the anticipatory logistics trend, predictive algorithms based on an increasing amount of data about demand and transport services can enable LSPs to improve their efficiency and service quality. In the logistics marketplace trend, a public and online overview of services and their characteristics (e.g., rates, schedules, durations) functions as a market for shippers to satisfy their freight demand for individual orders. This will result in flexible transport tailored to the shipper needs at specific moments when freight arrives. In the most futuristic trend, the supergrid logistics, orchestrators of logistics services will connect production, storage, and transport stakeholders electronically. In these three trends, anticipatory synchromodal scheduling can be used to, for example, evaluate future performance of different combinations of logistic services, taken from the digital logistics marketplace, and select the best ones considering the latest circumstances. 


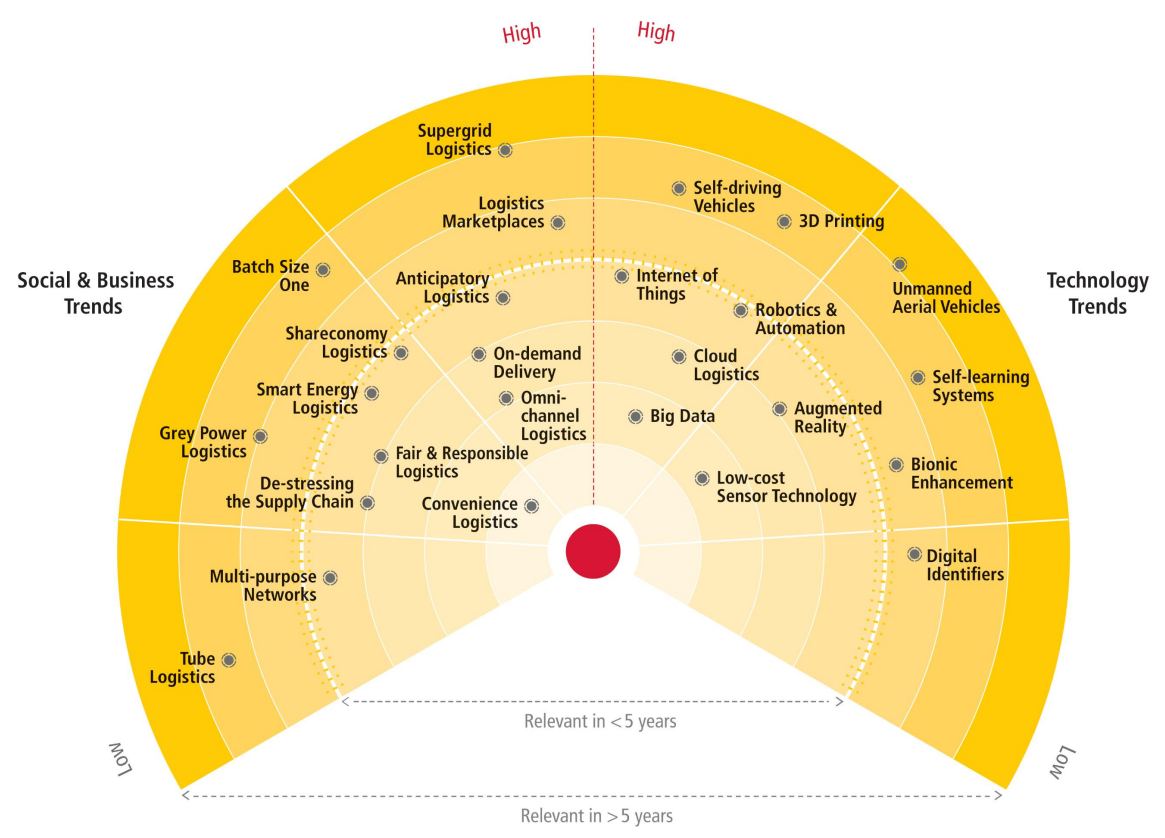

Figure 1.5: DHL Trend Radar 2016 [24]

In the aforementioned envisioned logistics, the digital, interconnected, and flexible networks will nurture the deployment of the synchromodality paradigm and the anticipatory scheduling within. The capability to implement such a logistics management paradigm is becoming increasingly closer thanks to the rapid development of information technologies. However, the complexity of the day-to-day decisions and the range of their impact is also increasing with the availability of information and wider perspective on performance. Traditional methods for scheduling multi-modal transport may fall short. In the following section, we briefly describe the characteristics of methods for scheduling multi-modal freight transport from the scientific literature, and their relation to synchromodality. We analyze aspects that can be useful for anticipatory scheduling in synchromodality and shortcomings that need improvement. These aspects and shortcomings are used to motivate our research design and research questions.

\subsection{Theoretical Motivation}

Anticipatory scheduling of freight in synchromodal transport requires the analysis of the current and near-future network conditions, the construction and evaluation of alternative decisions, and the appropriate timing for making the best decisions. The discipline that studies such analysis and optimization of decision problems is Operations Research (OR). 
The field of OR focuses on the mathematical modeling of problems to analyze them, and the design of algorithms to solve the models. From a mathematical modeling perspective, synchromodal transport is an extension of intermodal and multi-modal transport [92]. Although some parts of a synchromodal transport process can already be handled by current models for multi-modal transport (e.g., routing, dispatching, and assignment models), some aspects are lacking or need further improvement. In this section, we briefly discuss those aspects and exemplify the gaps of scheduling models for multi-modal transport with respect to synchromodality. We mainly focus on models studied in the last decade, although it is important to note that the OR community has actively investigated models for multi-modal transport and sequentially built knowledge since the early 1990s [19, 116]. The high-level discussion in this section is the motivation for our project, research design and research questions presented later on in Section 1.3. We leave out of this section an in-depth discussion of the technical aspects of the models, as well as their solution algorithms, and re-take it later on in this thesis, specifically in Sections 2.2, 4.3, 5.2, and 6.2, where we also present additional literature relevant to each part of the thesis. For an overview of research about planning and scheduling multi-modal transport, we refer to the reviews of SteadieSeifi et al. [92] and Mar Agamez-Arias and Moyano-Fuentes [56].

The models which are most closely related to anticipatory scheduling in synchromodality are those used for tactical and operational planning problems in multi-modal transport. The objective of tactical planning problems in multi-modal transport has been to optimize the use of the given infrastructure by choosing services, assigning orders, and determining itineraries [92]. The tactical problem that closely relates to synchromodality is Dynamic Service Network Design (DSND). Decision problems in DSND involve the choice of transport services and transhipment for freight, over a multi-period horizon, where at least one problem characteristic varies over time [92]. Although DSND research relates to some of the aspects of synchromodality, such as the time-space evolution of the network, two of the shortcomings in most DSND studies with respect to synchromodality are that: (i) they do not incorporate all time aspects such as time-windows and information about pre-announced orders [19], (ii) they do not incorporate multiple-modes or the flexibility to change them at any time, and (iii) they typically assume deterministic demand [92]. Although there are exceptions to these shortcomings, models seem to focus on one exception at a time and leave out the rest. For example, studies that model some time and flexibility aspects, such as Andersen, Crainic, and Christiansen [2] and Moccia et al. [63], or that consider information that becomes known over time, such as Li, Negenborn, and Schutter [51] and Nabais et al. [65], do not explicitly incorporate probability distributions to capture uncertainty in demand. Models that incorporate uncertainty in the form of random variables, such as Lium, Crainic, and Wallace [52], yield one initial plan that is robust to all realizations of the random variables as opposed to a dynamic (re-)planning policy. The few models that incorporate randomness, such as Bai et al. [4] and Lo, An, and Lin [53], and that include both planning and re-planning in a two-stage approach, usually consider a single transport mode.

The objective of operational planning problems in multi-modal transport has mostly been to cope with the real-time adjustments necessary to react to any kind of disturbance in the tactical solution [92]. In contrast to tactical planning problems, operational problems have 
been studied significantly less [92]. Furthermore, research concerning dynamic and stochastic freight transport planning has been studied extensively for a single mode [75, 78]. For example, models that consider random demand and random traveling times, such as [100, 99], usually consider a single type of resource or a "fleet" type. The required extension is to consider heterogeneous or substitutable resources, as shown in [101]. Considering that synchronization of operations might fail if uncertainty is ignored [92], this is a gap that must be filled for synchromodality to succeed. Another problem encountered in operational planning models is their focus on a single-objective rather than a multi-objective goal, especially when recognized objectives may be conflicting with each other [92]. For example, in the area of drayage operations, which on itself has received limited research attention [13], the major focus has been on "one plan" per day and the minimization of trucking costs instead of the optimization of networkwide performance [27]. Finally, only a limited number of papers have modeled the real-time aspects and the timing of the decision process and have further integrated different levels of planning, which may provide more flexible and sustainable solutions for the logistics industry [92].

The examples above show that aspects of synchromodality such as choosing services, assigning orders, and stochastic demand have been studied separately in the scientific literature. Within DSND, there has been little consideration about large stochastic multiperiod problems $[52,92,115]$. Within dynamic and stochastic freight transport, research about multiple modes has been studied less in comparison to a single mode [7, 75]; and decision making has been more single than multi-objective oriented [27, 92]. Furthermore, reviews of multi-modal freight transport planning point out that research on the integration of different planning problems is necessary for the adoption of mode-free booking and network-wide view (i.e., synchromodality) by LSPs $[92,56]$. Altogether, the state-of-the-art scientific literature provides a base for synchromodality and gives guidelines regarding the challenges that deserve further research attention. These research guidelines and the gaps just described motivate our research design, which we present in the following section.

\subsection{Research Design}

Our research is embedded within the project SynchromodalIT funded by the Dutch Institute for Advanced Logistics (DINALOG). In this project, there is a consortium of academic, IT, and LSP partners that are involved in technical and practical issues of synchromodal transport planning in The Netherlands. The project combines practical experience from industry with scientific knowledge from academia to investigate how synchromodal IT systems can improve the efficiency and sustainability of freight transport in a multi-modal network. The project aims to design synchromodal logistic network models, integrated service platforms, and planning and scheduling policies through the combination of operations research techniques and information technologies. It is divided into three research lines, each with a $\mathrm{PhD}$ project. The first line is about the real-time and big data aspects of synchromodal transport and is concerned with the static and dynamic aspects of data management as well as the analysis and predictions about the state of the transport network. The second line is about the planning of synchromodal transport and is concerned with the design of quantitative models that characterize relations between demand patterns, real-time 
decisions, and logistical costs as well as planning algorithms that support decision-making. The third line is about IT architectures for businesses in synchromodal transport and is concerned with the integration of information and services through a service platform.

Our work corresponds to the second research line of the SynchromodalIT project, the planning of synchromodal transport, specifically the anticipatory scheduling of freight transport processes. Although IT developments are increasingly enabling the analysis of potential network conditions (e.g., reducing uncertainty about future circumstances), as well as a seamless network-wide control of transport processes, the state-of-the-art scheduling methods for multi-modal transport still need further development for making anticipatory decisions. As mentioned before, traditional scheduling approaches focus on a single and instantaneous objective in a static network. This focus must change into a multi-objective, anticipatory, and dynamic perspective. To improve the scheduling of freight transport in multi-modal networks towards synchromodality, we define the main research objective of this thesis as follows.

We aim to develop mathematical models and heuristic algorithms that support anticipatory freight scheduling in synchromodal transport, to evaluate the output of our models and algorithms in terms of logistical costs, and to gain insights into the benefits of anticipatory decision making under different synchromodal transport networks.

The similarities between multi-modal and synchromodal transport permit some of the scheduling methods of the former to be used as a starting point for synchromodal scheduling. The differences, however, require more than just extending the existing scheduling methods. The need for enhancements on such methods accentuate when considering that performance in synchromodality is measured on a network-wide, dynamic, and multi-period manner. To design anticipatory scheduling methods in synchromodal transport and investigate their benefits in logistical performance, we define the following research questions and approach.

\subsubsection{Research Questions}

Our research goal relates to the improvement of multi-modal transport through scheduling methods for synchromodality. In this thesis, a scheduling method refers to the combination of a mathematical model and a heuristic algorithm whose outcome is a decision about the freights and transport services at a given point in time. To develop and evaluate such scheduling methods, as well as to gain insight into their use, we define the following Research Questions (RQs):

RQ 1. What are the limitations of current scheduling methods for multi-modal transport with respect to synchromodality and what improvements are necessary?

We study the academic literature about scheduling methods for different parts of multimodal transport. We examine their advantages and disadvantages with respect to the core characteristics of synchromodality, and determine what improvements are necessary for the scheduling of synchromodal transport. 
RQ 2. How can anticipatory scheduling methods be designed for different parts of a synchromodal transport network?

Parts of a multi-modal transport network vary in type and breadth of scheduling decisions. Due to synchromodality, new efficiency opportunities may occur in different parts. We investigate such opportunities and study how anticipatory scheduling methods can achieve them. We engage on the limitations of the optimization models from academic literature for those parts, and study how to overcome them.

RQ 3. How can the anticipatory scheduling methods of $R Q 2$ be modified to handle large size instances efficiently?

Optimization models have computational limitations for large problem instances. For such instances, heuristics are used. Due to the dynamic and prolonged nature of synchromodality, and the difference in execution time and planning stages of different transport processes in a multi-modal network, different heuristic mechanisms may be required for anticipatory scheduling methods concerning different parts of the network. We investigate how anticipation of future network conditions can be incorporated in heuristics for the decision of postponing, executing, interchanging, or stopping, the transport of a freight.

RQ 4. How do anticipatory scheduling methods perform under different network settings and how do they compare to other benchmarks?

The realization of uncertainties in the freight transport process may result in gains or losses, even when anticipatory decisions were made. We explore the influence of different synchromodal network settings such as demand patterns, time-windows, and service capacity in the performance of anticipatory scheduling decisions. Furthermore, we compare the performance of our scheduling methods against benchmarks from practice and literature to gain insights into the strengths and weaknesses of our methods.

RQ 5. How can serious gaming be used to facilitate the adoption of anticipatory scheduling methods for synchromodal transport in practice?

Serious gaming is a tool commonly used in the active training of personnel for a new task too complex or expensive to train in practice. We explore how to use this tool to raise awareness on the vital workings of our scheduling methods, i.e., anticipation of future freight and dynamic changes in a freight's plan, and to facilitate their understanding and adoption by practitioners.

To answer these research questions within reasonable time, we break down a multi-modal transport network into several parts and make a number of assumptions that limit our scope. Furthermore, we use a research methodology based on techniques from operations research, discrete event simulation, and serious gaming. We now explain our research approach in more detail. 


\subsubsection{Research Approach}

To study the various aspects of synchromodality, we divide a multi-modal transport network into three separate parts and one combined, as shown in Figure 1.6. Studying the network using three separate parts has two advantages. The first advantage is that we can isolate some of the synchromodality characteristics that only affect specific segments of the network and study their influence on scheduling decisions. Consider, for example, the long-haul transport of freight performed by a daily train. The decision studied could be whether to consolidate or postpone the transport of freight. In another example, consider drayage transport (i.e., first- and last-mile of freight) performed by trucks. The decision studied could be how to route the trucks and assign a terminal for freight that will continue on the long-haul. The second advantage is that the division allow us to use existing literature on each part, as well as the experience and data from our industry partners in the project that focus on different parts of the network. Thus, our designs can be motivated by real-life cases, built upon state-of-the-art methods, and studied using representative scenarios motivated by industry. Besides these advantages, the division of a transport network shown in Figure 1.6 is also motivated by a consortium partner that transports containers, on a daily basis, from the Eastern part of the country to the port of Rotterdam, and back. This LSP has uses barges, trains, and trucks distributed among four intermodal terminals.

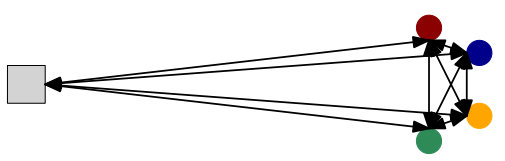

Part 1. Long-haul round-trip transport.

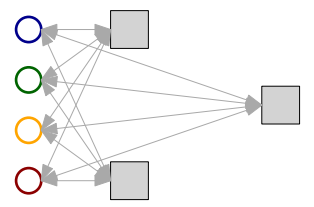

Part 3. Multi-terminal drayage transport.

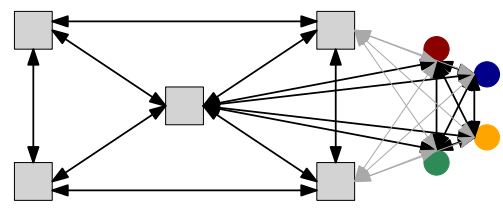

Part 2. Long-haul multi-transfer transport.

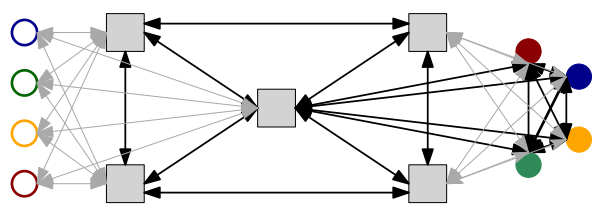

Part 4. Integrated long-haul and drayage transport.

Legend: $\square$ Terminals $\mathrm{O} \bigcirc \mathrm{O}$ Origins

Destinations

$\longrightarrow$ Alternative mode

$\longleftrightarrow$ High-capacity modes

Figure 1.6: Parts of a synchromodal transport network studied separately and combined.

Even though the parts of the network studied differ in the extent of their transport processes and the constraints on the scheduling decisions, we assume two scheduling characteristics hold for all parts. First, we consider that a single decision maker has the overview and control of all processes and transport services in the corresponding part of the synchromodal network, even though the decision maker might not own these. Second, we consider that the capacity of all processes and services is expressed in the same discrete unit, such as the Twenty-foot Equivalent Unit (TEU). We refer to this unit as a freight. 
We further discuss these assumptions and all scheduling characteristics for each part of the network in their corresponding chapters, which we introduce in Section 1.4.

Our methodology for answering RQs 1 through 3 is based on Operations Research (OR) techniques used for mathematical modeling and analysis and optimization of industrial processes. Some of the OR modeling techniques we use include integer linear programming to model transport networks and Markov decision processes to model decision making under uncertainty. Some of the OR algorithmic techniques include local-search heuristics and approximate dynamic programming to solve large scale models. For answering RQ 4, we use simulation. This technique allows us to compare our scheduling methods against benchmark methods from theory and from practice in a controlled and reproducible way. For RQ 5, we use serious gaming to convey the workings and benefits of our scheduling methods into practice in a pedagogical and entertaining way. In the following section, we present an outline of the remaining chapters of this thesis and their relation to our research questions and approach.

\subsection{Thesis Outline}

In this section, we outline the contents of the six research chapters of this thesis. The correspondence between the four parts of the synchromodal transport network and the chapters is shown in Figure 1.7. The relation between the research questions and chapters, as well as their related publications, is shown in Figure 1.8. Note that, due to our division of the network, some of the research questions are answered in multiple chapters. In the following, we briefly describe the contents, the research questions addressed, and the methodology used in each chapter.

In Chapter 2, we describe the problem of scheduling transport of freight on single-trips and round-trips of one high-capacity mode with the availability of a low-capacity alternative mode. This problem is similar to the one exemplified in Section 1.1.1. We consider a multi-period cost minimization setting where there is uncertainty in the freights that arrive each period, and their characteristics (e.g., time-windows, destination, etc.). We study how to model the underlying stochastic finite horizon optimization problem as a Markov Decision Process (MDP) and how to design a heuristic algorithm based on Approximate Dynamic Programming (ADP) to solve large problem instances. We provide insights about the design of basis functions in ADP and their evaluation.

In Chapter 3, we study the use of the scheduling method developed in Chapter 2, i.e., the MDP model and ADP algorithm, under different synchromodal networks settings in a series of simulation experiments. These settings describe different LSPs, as well as different situations in day-to-day workings within a single LSP. We provide managerial insights into the gains of using our approach under different initial conditions and demand patterns, as well as a discussion on the practical applicability of our scheduling method. 


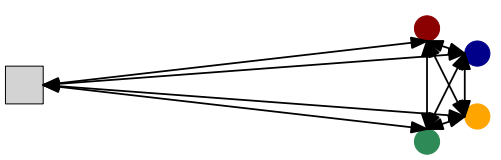

Chapters 2, 3, and 7: Scheduling long-haul transport of freights to different locations within one region, in a highcapacity mode traveling single-period round-trips.

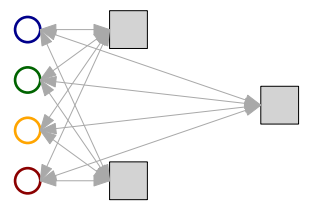

Chapter 5: Scheduling pre- and end-haulage transport of freights in trucks, considering terminal assignment for the pre-haulage freights (i.e., start of the long-haul).

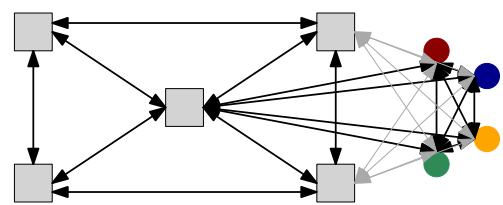

Chapter 4: Scheduling long-haul transport of freights in multiple high-capacity modes traveling multi-period routes with possible transfers.

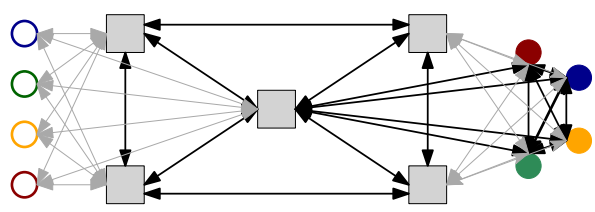

Chapter 6: Integrated scheduling of drayage operations (i.e., pre- and end-haulage transport) and long-haul transport for freights in a synchromodal network.

Legend: $\square$ Terminals $\bigcirc \bigcirc \bigcirc \bigcirc$ Origins $\bigcirc \bigcirc \bigcirc$ Destination

$\longrightarrow$ Alternative mode High-capacity modes

Figure 1.7: Parts of the synchromodal transport network analyzed in the different chapters.

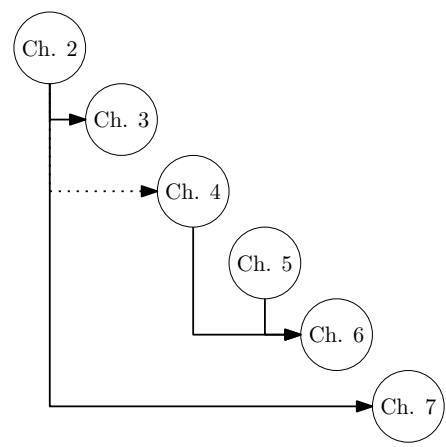

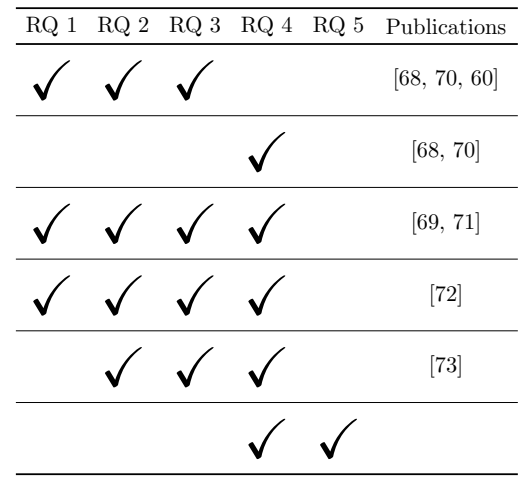

Figure 1.8: Relation between the chapters and research questions of this thesis. The publications this thesis is based on are [69, 70, 71, 61, 72, 73, 74].

In Chapter 4, we describe the problem of scheduling freight in a multi-modal network with more than one high-capacity path (e.g., multiple services and multiple transfers) between origin and destination. We consider a multi-period horizon in which services and transfers may last for more than one period and where there is uncertainty in the freights that arrive each period and their characteristics. We consider the maximization of a generic reward function where rewards and costs are distributed through time. In a similar way to Chapter 2, we study how to model the complex space-time evolution of the stochastic optimization problem using MDP and Mixed- 
Integer Linear Programming (MILP) and how to design a heuristic algorithm that handles the evolution of decisions and rewards using ADP and Bayesian exploration. We provide design insights into the parametrization of ADP and the balance of the exploration vs. exploitation dilemma. We examine the performance of our scheduling method under different synchromodal transport networks using simulation experiments and provide practical insights. Furthermore, we discuss the challenges resulting from a more complex part of the network in comparison to the simpler part from Chapter 2 .

In Chapter 5, we describe the problem of scheduling drayage transport, also known as pre- and end-haulage transport. In synchromodality, the pre-haulage of freight does not necessarily have a fixed terminal, the end-haulage can be to a terminal or another customer, and both pre- and end-haulage can be re-scheduled as long as customers and terminal time-windows allow it. We consider a rich set of drayage characteristics and the minimization of vehicle routing and terminal assignment costs. We study how to model this deterministic optimization problem using MILP and how to design a Math-Heuristic ( $\mathrm{MH})$ that allows re-scheduling and that takes advantage of the flexibility in anticipation to future parts of the transport chain. We analyze the performance of our scheduling method under different network settings using simulation experiments and provide practical insights into the static and dynamic use of our approach.

In Chapter 6, we describe the problem of scheduling drayage operations and longhaul transport in a multi-service and multi-transfer network in an integrated way. We combine the problem characteristics of Chapters 4 and 5 into an integrated stochastic optimization problem. We study how to combine the MDP model and ADP algorithm of Chapter 4 with the MILP model and MH of Chapter 5 using an integrated simulation approach for dynamic and anticipatory scheduling over the entire network. We outline design challenges of integrating the scheduling of different parts of the network and study mechanisms to overcome them using simulation experiments. We analyze the performance of our integrated scheduling method under various network settings using simulation experiments and provide practical insights into the savings in networkwide logistical costs and its consequences for drayage operations and long-haul transport.

In Chapter 7, we describe how serious gaming can be used to raise awareness about the trade-off in the stochastic optimization problem from Chapter 2 and to educate on how anticipatory scheduling can optimize this problem. We design a single-player web-based game that simulates an LSP facing the problem in Chapter 2 and incorporate the solution algorithms developed therein. We provide insights into the use of serious gaming to facilitate the understanding and adoption of anticipatory scheduling ideas. 
In Chapter 8, we summarize the main findings of our research and the contributions to the academic literature. Furthermore, we recap the practical insights for the use and implementation of our anticipatory scheduling methods. We finalize by delineating directions for further research and reflecting upon the anticipatory scheduling of freight in synchromodal transport. 


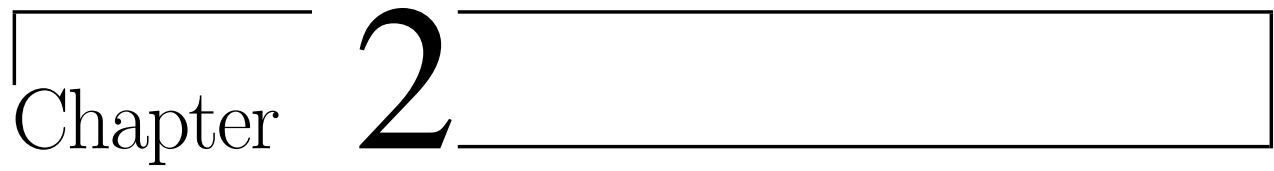

\section{Long-haul Round-trip Transport: Solution Design}

In this chapter, we consider the scheduling problem that LSPs face when transporting freights within their time-window using periodic long-haul round-trips. A long-haul round-trip consists of two parts: (i) delivery of freights from a single origin to different locations within a far away region using a high-capacity mode, and (ii) pick-up of freights from locations within the same region, which are not necessarily the same as the delivery locations, and subsequent transport back to the same origin in the same high-capacity mode. Although freights become known gradually over time, we assume there is probabilistic knowledge about their arrival and their characteristics at each period of the horizon. The goal of the LSP is to minimize the costs resulting from the locations visited by the high-capacity mode in the round-trip and the use of an alternative transport mode, over the time horizon. We model this optimization problem using Markov Decision Process (MDP) theory and design a heuristic solution using Approximate Dynamic Programming (ADP). We show different design challenges that arise when applying ADP to our problem and how these challenges can be overcome to provide accurate approximations to the optimal solution of the MDP model. This chapter is based on parts of our publications in $[69,71,61]$.

\subsection{Introduction}

The scheduling problem we address in this chapter is motivated by a Dutch LSP that transports containers from the Eastern part of the country to the Port of Rotterdam, and vice versa, in daily round-trips using a barge. Each day, this barge transports containers from a single inland terminal to different deep-sea terminals within the port. While delivering containers, the barge picks up containers from the same, and other terminals, and transports 
them back to the inland terminal where it started. Alternatively, the LSP has trucks to transport containers. The challenge consists on how to schedule the transport of new containers that arrive for both parts of the round-trip (i.e., using today's or tomorrow's barge or trucks), to achieve the best network performance over time.

Ideally, the barge would visit as few terminals in the port as possible and trucks would be seldom used. However, the variability in the amount and the type of containers that arrive each day makes the ideal situation hard to achieve. Each day, the LSP must choose which containers to consolidate in the barge of that day and which ones to postpone, in order for its operations to be close to optimal over time. For example, postponing the transport of a container to, or from, a given terminal today can reduce the number of terminals visited today without increasing the number of terminals to be visited tomorrow. Also, transporting a container that has a long time-window today can reduce the number of terminals that need to be visited tomorrow. A good balance of consolidation and postponement in each round-trip results in a good performance over time.

Legend:

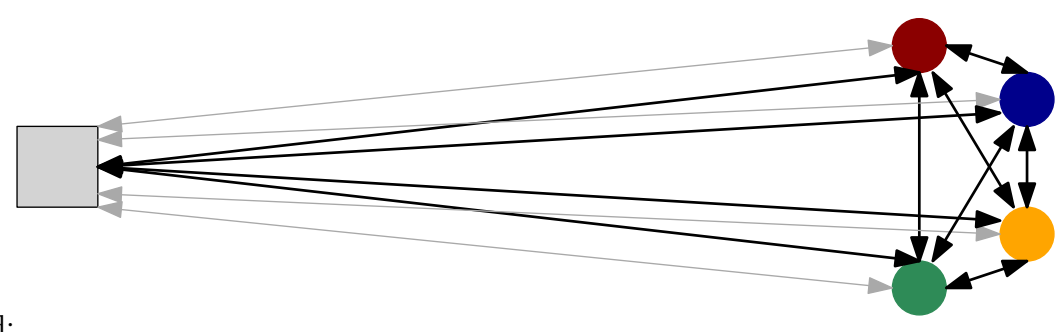

Origin $\bigcirc$ Destinations $\longrightarrow$ Alternative mode High-capacity mode

Figure 2.1: Example network for a round-trip

In general terms, we study the problem of scheduling the transport of freights in long-haul round-trips, periodically. In every period, one round-trip is performed, and in each round trip, freights are transported (i) from a single origin to multiple locations within a far away region, and (ii) from locations in that region back to the origin, using a high-capacity mode. The region is far away from the origin, but locations within the region are close to each other, as exemplified in Figure 2.1. As a result, the long-haul, or largest part of the trip where freights are consolidated, is the same in every round-trip. Differences in costs between two periods arise due to the locations visited in the round-trip corresponding to each period and the use of the alternative transport mode (e.g., trucks). The alternative mode is more expensive than the high-capacity mode per freight. New freights, with different characteristics, arrive each period. Each freight has a given destination, a release-day, and a due-day. Although the number of freights, and their characteristics, vary from period to period, there is information about their probability distribution. The objective of the decisions is to reduce the total costs over a multi-period horizon while transporting all freights.

Scheduling decisions in long-haul round-trips that minimize the costs over a multi-period 
horizon are complex for three reasons. First, the freights that arrive in each period are uncertain. The uncertainty is not only on the number of freights that arrive, but also on their characteristics. Second, the time-window of each freight restricts the periods in which it can be consolidated and to which it can be postponed. Third, the cost advantage of consolidating as many freights as possible in the high-capacity mode can be conflicting with the objective of reducing costs over a multi-period horizon. Our goal in this chapter and the next is twofold: (i) to design an anticipatory scheduling method that properly handles the aforementioned complexities, and (ii) to provide insight into the effects of various problem characteristics on the anticipatory freight selection decisions. The first goal is worked upon in this chapter, whereas the second goal is worked upon in the next chapter. Specifically, we work upon modeling the stochastic and time dependent nature of the optimization problem and designing a heuristic solution approach that is close to optimal in this chapter.

The remainder of this chapter is structured as follows. In Section 2.2, we briefly review the relevant literature and specify our contribution to it. In Section 2.3, we introduce the notation and formulate the problem as an MDP. In Section 2.4, we present our ADP solution approach. In Section 2.5 we present our experimental setup and various ADP designs. In Section 2.6, we evaluate our designs for the ADP algorithm and provide a comparison with the optimal values. In Section 2.7, we reflect upon the limitations of our approach. Finally, we conclude this chapter in Section 2.8 with theoretical insights about modeling the problem and designing a good solution algorithm.

\subsection{Literature Review}

Our problem is related to the vast literature on freight consolidation in multi-modal networks. In this brief review of it, we focus on two problem classes: (i) problems concerning assignment of freights to modes in a multi-modal network, and (ii) problems concerning anticipatory and dynamic selection of transport loads. In the first class, we summarize the key points and shortcomings of models and solution approaches proposed for Dynamic Service Network Design (DSND). In the second class, we provide examples on how the dynamic and stochastic nature of demand has been captured in routing and transport problems, and what kind of solutions have been proposed. For an extensive review on research about the first problem class, we refer the reader to Crainic and Kim [19] and SteadieSeifi et al. [92]; and for the second class, to Pillac et al. [75] and Powell, Bouzaiene-Ayari, and Simao [78].

Decision problems in DSND involve the choice of transport services for freight, over a multi-period horizon, where at least one problem characteristic varies over time [92]. However, two of the shortcomings in most DSND studies are that: (i) they do not incorporate time dependencies such as time-windows and information about pre-announced orders [19], and (ii) they assume deterministic demand [92]. Furthermore, it seems that studies that tackle these shortcomings do so one at a time. For example, studies that model time dependencies, such as Andersen, Crainic, and Christiansen [2], and consolidation opportunities, such as Moccia et al. [63], assume deterministic demand. Recently, optimization studies that model multiple time dependencies in multi-modal networks, such as Li, Negenborn, and Schutter [51] and Nabais et al. [65], use approaches based on receding horizons and model predictive 
control to take advantage of information that becomes known over time. Although these two studies do not explicitly incorporate probability distributions to capture uncertainty, they establish the benefits of including dynamic information in optimization models. Research that models uncertainty in the demand, such as Hoff et al. [43], is usually developed for a single mode. Furthermore, models that incorporate random variables, such as Lium, Crainic, and Wallace [52], yield one initial plan that is robust to all realizations of the random variables. Only a few of these models, such as Bai et al. [4] and Lo, An, and Lin [53] include both planning and re-planning of a single transport mode, in a two-stage approach.

One of the reasons why the shortcomings above have been tackled one at a time lies in the solution approaches used. Graph theory and meta-heuristics, which have been often proposed to solve DSND problems [92, 115], are less suitable for dealing with time-dependencies and stochastic demands. To deal with time-dependencies, mathematical programming techniques such as cycle-based variables [1], branch-and-price [3], digraphs formulations [63], and decompositions [36] have been used. However, these techniques are computationally expensive. Consequently, meta-heuristics, such as those based on Tabu Search [16, 112], have been used for larger problems [92]. Integrating stochasticity in these techniques and heuristics requires additional designs, such as stochastic scenarios [43], or two-stage stochastic approaches $[4,53]$. Increasingly, the potential gains of integrating stochasticity have been recognized in practice [52] and in theory [119].

In contrast to the first problem class, the second class concerning dynamic and stochastic freight transport has been studied extensively for single mode routing [75, 78]. Although our problem contains multiple modes, research done in this second class provides valuable insights. For example, knowing orders one or two days in advance has been shown to improve the performance of trucking companies [118]. To model stochastic demand that is revealed dynamically over time, two strategies have been commonly applied: (i) sampling strategies, and (ii) stochastic modeling [75]. Both strategies yield solutions that anticipate on the realization of the stochastic variables and that perform better than non-anticipatory approaches. An example that combines all of the insights above is Amazon's anticipatory package shipping [91]. This patent describes the combination of information about existing and historical orders with forecasts of future orders, to transport pre-assembled packages to intermediate warehouses and even to trucks for the so-called "same day" deliveries. In our case, anticipatory decisions include two alternatives, either consolidating or postponing freight for better performance in the future.

The previous paragraph exemplifies the trend of using dynamic and probabilistic information in logistics and transport decisions. The need for further research that includes probabilistic knowledge in the planning of dynamic transport problems has been outlined [15]. However, using such information, under the sampling and stochastic modeling strategies, comes with various difficulties. Sampling methods come with some form of bias and are heuristic in nature, such as the Indifference Zone Selection approach used by Ghiani et al. [38]. Stochastic modeling requires analytical models of the evolution of the system and its variability, which are usually non-scalable to real-life instances, such as the MDP model used by Novoa and Storer [67]. To overcome the difficulties of each strategy, several techniques have been proposed [75]. To reduce the bias of sampling methods, Multiple Scenario Approaches 
with algorithms based on consensus, expectation, or regret of the probabilistic knowledge, have shown significant benefits [6]. To reduce the dimensionality issues of stochastic modeling, ADP based on roll-out procedures and value function approximation has been used $[67,86]$.

Summarizing, research about multi-modal and stochastic freight transport has had different perspectives. Within DSND, there has been little research about large stochastic multi-period problems $[52,92,115]$. Within dynamic and stochastic freight transport, research about multiple modes and round-trips has been studied less in comparison to a single mode $[7,75]$. This chapter deals with multi-period stochastic cost minimization through anticipatory decisions to schedule freight in high-capacity mode for long-haul round-trips considering an alternative mode. For these reasons, we believe our work has three contributions to the existing literature. First, we propose an MDP model that includes stochastic freight demand and its characteristics for a multi-modal network, handles complex time-dependencies, and measures performance over a multi-period horizon. Second, we propose an ADP algorithm to solve the model for large problem instances. Third, we provide methodological insights on the design process of an ADP algorithm for our problem.

\subsection{Mathematical Model}

In this section, we formulate a model of the optimization problem described in Section 2.1 using MDP theory. First, we introduce the notation for the problem characteristics. Next, we formulate the stages, states, decisions, transitions, and the optimality equations of the MDP model. Finally, we discuss the dimensionality issues of this model.

\subsubsection{Notation}

We consider a multi-period horizon $\mathcal{T}=\left\{0,1,2, \ldots, T^{\max }-1\right\}$. At each period $t \in \mathcal{T}$, one high-capacity mode performs a round-trip, traveling from a single origin to a group of locations $\mathcal{D}^{\prime} \subseteq \mathcal{D}$ within a region $\mathcal{D}$, and back. Freights transported by this vehicle are categorized in two types: (i) delivery and (ii) pickup freights. Delivery freights are those transported from the origin to a location $d \in \mathcal{D}$ and pickup freights are those transported from a location $d \in \mathcal{D}$ back to the origin. Since only one round-trip is planned each period, a total of $T^{\max }$ consecutive round-trips are considered. Each period, the planner selects which of the released freights, of both types, to consolidate in that round-trip. For simplicity, we refer to a period as a "day", and to a delivery or pickup location as a "destination".

Each freight must be transported within its own time-window. Time-windows are characterized by a release-day $r \in \mathcal{R}=\left\{0,1,2, \ldots, R^{\max }\right\}$ and a time-window length of $k \in \mathcal{K}=\left\{0,1,2, \ldots, K^{\max }\right\}$ days. For modeling purposes, the release-day is relative to the current-day and the time-window length is relative to the release-day. For example, a freight that has $r=1$ and $k=0$ today will be released tomorrow and must also be transported tomorrow. Note that $r$ is the number of days in advance that the LSP knows about a freight before it can be transported. Also note that $k$ is the number of days within which the LSP must transport a freight, once it has been released. 
Although freights are known only after they arrive, the LSP has probabilistic knowledge about them in the form of eight probability distributions. In between two consecutive days, $f \in \mathcal{F}$ delivery freights and $g \in \mathcal{G}$ pickup freights arrive with probability $p_{f}^{\mathrm{F}}$ and $p_{g}^{\mathrm{G}}$, respectively. A freight has destination $d \in \mathcal{D}$ with probability $p_{d}^{\mathrm{D}, \mathrm{F}}$ in case of delivery, and $p_{d}^{\mathrm{D}, \mathrm{G}}$ in case of pickup. A freight has release-day $r \in \mathcal{R}$ with probability $p_{r}^{\mathrm{R}, \mathrm{F}}$ in case of delivery, and $p_{r}^{\mathrm{R}, \mathrm{G}}$ in case of pickup. A freight has time-window length $k \in \mathcal{K}$ with probability $p_{k}^{\mathrm{K}, \mathrm{F}}$ in case of delivery, and $p_{k}^{\mathrm{K}, \mathrm{G}}$ in case of pickup.

In each period, two transport modes are available. First, there is one high-capacity mode doing the round-trip, with capacity of $Q$ delivery freights and $Q$ pickup freights. The costs $C_{\mathcal{D}^{\prime}}$ of this vehicle depend on the group of destinations $\mathcal{D}^{\prime} \subseteq \mathcal{D}$ that it visits . In addition to $C_{\mathcal{D}^{\prime}}$, there is a cost $B_{d}$ per freight with destination $d$ consolidated in the high-capacity mode. Second, we assume there is an unlimited number of alternative modes (e.g., trucks) for urgent freights, i.e., released freights whose due-day is immediate $(r=k=0)$, at a cost of $A_{d}$ per freight to or from destination $d$. The restriction of using alternative vehicles only for urgent freights does not impact the decision making process since we assume that there are no holding costs and that transport costs do not change over time. We introduce this restriction to reduce the size of the decision space, and thus the computational complexity of the model. We do not consider holding (i.e., inventory costs) since our focus is on the long-haul round-trip decisions, not on the pre- and end-haulage operations, and the time-window lengths are a tighter restriction on the postponement decisions than the physical space.

\subsubsection{Formulation}

Each day corresponds to a stage in the MDP. Thus, stages are discrete, consecutive, and denoted by $t$. At each stage $t$, there are $F_{t, d, r, k}$ delivery freights and $G_{t, d, r, k}$ pickup freights with destination $d$, release-day $r$, and time-window length $k$. The state of the system $S_{t}$ consists of all freight variables at stage $t$, as seen in (2.1). We denote the state space of the system by $\mathcal{S}$, i.e., $S_{t} \in \mathcal{S}$.

$$
S_{t}=\left[\left(F_{t, d, r, k}, G_{t, d, r, k}\right)\right]_{\forall d \in \mathcal{D}, r \in \mathcal{R}, k \in \mathcal{K}}
$$

At each stage $t$, the scheduling decision consists of which delivery and pickup freights from $S_{t}$ to consolidate in the high-capacity mode. This decision is restricted by the release-day of freights and by the capacity of this mode. We use the non-negative integer variables $x_{t, d, k}^{\mathrm{F}}$ and $x_{t, d, k}^{\mathrm{G}}$ to represent the number of released freights with destination $d$ and time-window length $k$ consolidated, for delivery and pickup freights respectively. The decision $x_{t}$ consists of all decision variables at stage $t$ as seen in (2.2a), subject to constraints (2.2b) to (2.2f), which define the feasible decision space $\mathcal{X}_{t}$.

$$
\begin{gathered}
x_{t}=\left[\left(x_{t, d, k}^{\mathrm{F}}, x_{t, d, k}^{\mathrm{G}}\right)\right]_{\forall d \in \mathcal{D}, k \in \mathcal{K}} \\
\text { s.t. } \\
0 \leq x_{t, d, k}^{\mathrm{F}} \leq F_{t, d, 0, k}, \forall d \in \mathcal{D}, k \in \mathcal{K}
\end{gathered}
$$




$$
\begin{gathered}
0 \leq x_{t, d, k}^{\mathrm{G}} \leq G_{t, d, 0, k}, \forall d \in \mathcal{D}, k \in \mathcal{K} \\
\sum_{d \in \mathcal{D}} \sum_{k \in \mathcal{K}} x_{t, d, k}^{\mathrm{F}} \leq Q, \\
\sum_{d \in \mathcal{D} k \in \mathcal{K}} \sum_{t, d, k} \leq Q, \\
x_{t, d, k}^{\mathrm{F}}, x_{t, d, k}^{\mathrm{G}} \in \mathbb{Z}^{+} \cup\{0\}
\end{gathered}
$$

The costs of a decision depend on the destinations visited with the high-capacity mode and the use of the alternative mode. We define $y_{t, d} \in\{0,1\}$ as the binary variable that gets a value of 1 if destination $d$ is visited by the high-capacity mode at stage $t$ and 0 otherwise. We define $z_{t, d}$ as the variable representing the number of freights to and from destination $d$ that were transported with the alternative mode. These variables depend on the state and decision variables, as seen in $(2.3 \mathrm{~b})$ and $(2.3 \mathrm{c})$. Using these variables, the costs at stage $t$ can be defined as a function of $x_{t}$ and $S_{t}$, as shown in (2.3).

$$
\begin{aligned}
& C\left(S_{t}, x_{t}\right)=\sum_{\mathcal{D}^{\prime} \subseteq \mathcal{D}}\left(C_{\mathcal{D}^{\prime}} \cdot \prod_{d^{\prime} \in \mathcal{D}^{\prime}} y_{t, d^{\prime}} \cdot \prod_{d^{\prime \prime} \in \mathcal{D} \backslash \mathcal{D}^{\prime}}\left(1-y_{t, d^{\prime \prime}}\right)\right) \\
& +\sum_{d \in \mathcal{D}} \sum_{k \in \mathcal{K}}\left(B_{d} \cdot\left(x_{t, d, k}^{\mathrm{F}}+x_{t, d, k}^{\mathrm{G}}\right)\right)+\sum_{d \in \mathcal{D}}\left(A_{d} \cdot z_{t, d}\right) \\
& \text { where } \\
& y_{t, d}=\left\{\begin{array}{ll}
1, & \text { if } \sum_{k \in \mathcal{K}}\left(x_{t, d, k}^{\mathrm{F}}+x_{t, d, k}^{\mathrm{G}}\right)>0 \\
0, & \text { otherwise }
\end{array}, \forall d \in \mathcal{D}\right. \\
& z_{t, d}=F_{t, d, 0,0}-x_{t, d, 0}^{\mathrm{F}}+G_{t, d, 0,0}-x_{t, d, 0}^{\mathrm{G}}, \forall d \in \mathcal{D}
\end{aligned}
$$

The objective is to minimize the costs over the entire planning horizon, i.e., the sum of (2.3) over all $t \in \mathcal{T}$. However, there is uncertainty in the arrival of freights within this horizon, and thus in the states. Consequently, the formal objective is to minimize the expected costs over the horizon. Since for every possible state there is an optimal decision, and we do not know which states we will encounter at each stage, the solution to the objective has to be a group of decisions rather than a single one. We define a policy $\pi \in \Pi$ as a function that maps each state $S_{t} \in \mathcal{S}$ to a decision $x_{t}^{\pi} \in \mathcal{X}_{t}$, for every $t \in \mathcal{T}$. The goal is to find the policy $\pi^{*} \in \Pi$ that minimizes the expected costs over the planning horizon, given an initial state $S_{0}$, i.e., initial conditions, as seen in (2.4).

$$
\min _{\pi \in \Pi} \mathbb{E}\left\{\sum_{t \in \mathcal{T}} C\left(S_{t}, x_{t}^{\pi}\right) \mid S_{0}\right\}
$$

Using Bellman's principle of optimality, the optimal costs can be computed through a set of recursive equations. These recursive equations are expressed in terms of the current-stage 
and the expected next-stage costs, as seen in (2.5). Before solving these equations, we need to define how the system evolves over time, or in other words, the transition from $S_{t}$ to $S_{t+1}$.

$$
V_{t}\left(S_{t}\right)=\min _{x_{t} \in \mathcal{X}_{t}}\left(C\left(S_{t}, x_{t}\right)+\mathbb{E}\left\{V_{t+1}\left(S_{t+1}\right)\right\}\right), \forall S_{t} \in \mathcal{S}
$$

The transition from $S_{t}$ to $S_{t+1}$ is influenced by $x_{t}$ and by the freights that arrive after this decision. Remind that arriving freights, and their characteristics, are stochastic and characterized by eight probability distributions. To model this arrival process, we introduce the variables $\widetilde{F}_{t, d, r, k}$ and $\widetilde{G}_{t, d, r, k}$ to represent the delivery and pickup freights, respectively, that arrived between stages $t-1$ and $t$ having destination $d$, release-day $r$, and time-window length $k$. Note that we defined these variables with respect to stages $t-1$ and $t$, such that at $t$ all information is known. The exogenous information $W_{t}$ at stage $t$ consists of all the "new information", represented by $\widetilde{F}_{t, d, r, k}$ and $\widetilde{G}_{t, d, r, k}$, as seen in (2.6).

$$
W_{t}=\left[\left(\widetilde{F}_{t, d, r, k}, \widetilde{G}_{t, d, r, k}\right)\right]_{\forall d \in \mathcal{D}, r \in \mathcal{R}, k \in \mathcal{K}}
$$

A state $S_{t}$ at stage $t$ occurs as the result of the state of the previous stage $S_{t-1}$, the decision of the previous stage $x_{t-1}$, and the exogenous information $W_{t}$ that became known between the stages. Remind that, to model the time-windows of freights, release-days $r$ are indexed relative to $t$ and time-window lengths $k$ are indexed relative to $r$. This means that, if a freight has not been released (i.e., $r>0$ ), its due-day is $r+k$. Naturally, when a freight is released, its due-day is equal to its time-window length, and after that period, it must decrease by one every day that passes until the freight is transported. All of these factors, and index relations, are used to capture the transition of the system. We represent them using the transition function $S^{\mathrm{M}}$ shown in (2.7), which works as follows.

The transition of delivery (i.e, $F_{t, d, r, k}$ ) and pickup (i.e, $G_{t, d, r, k}$ ) freights with destination $d$, release-day $r$, and time-window length $k$, from $S_{t-1}$ to $S_{t}$, is defined having four considerations. First, freights that are released at day $t$ and have a time-window length $k<K^{\text {max }}$, are the result of freights from the previous day $t-1$ that were already released, had a time-window length $k+1$, and were not consolidated in the previous round-trip; in addition to freights from the previous day $t-1$ that had a next day release and the same time-window length $k$, and the freights that arrived between the previous and the current-day with releaseday 0 and time-window length $k$, as seen in (2.7b) and (2.7c). Second, freights that are already released at day $t$ and have a time-window length $k=K^{\max }$ are the result of freights from the previous day $t-1$ that had a next day release and the same time-window length $K^{\mathrm{max}}$, in addition to the freights that arrived between the previous and the current-day with the same characteristics, as seen in (2.7d) and (2.7e). Third, freights that are not released at day $t$, do not have the maximum release-day, i.e., $0<r<R^{\max }$, and have time-window length $k$, are the result of freights from the previous day $t-1$ that had a release-day $r+1$ and a time-window length $k$, in addition to the freights that arrived between the previous and the current-day with the same characteristics, as shown in (2.7f) and (2.7g). Fourth, freights that are not released at day $t$, have the maximum release-day $R^{\max }$, and have time-window length $k$, are the result only of the freights that arrived between the previous and the 
current-day with release-day $R^{\max }$ and time-window length $k$, as seen in (2.7h) and (2.7i).

$$
\begin{aligned}
& S_{t}=S^{\mathrm{M}}\left(S_{t-1}, x_{t-1}, W_{t}\right), \forall t \in \mathcal{T} \mid t>0 \\
& \text { where } \\
& F_{t, d, 0, k}=F_{t-1, d, 0, k+1}-x_{t-1, d, k+1}^{\mathrm{F}}+F_{t-1, d, 1, k}+\widetilde{F}_{t, d, 0, k}, \quad \forall d \in \mathcal{D}, k \in \mathcal{K} \backslash K^{\max } \\
& G_{t, d, 0, k}=G_{t-1, d, 0, k+1}-x_{t-1, d, k+1}^{\mathrm{G}}+G_{t-1, d, 1, k}+\widetilde{G}_{t, d, 0, k} \quad \forall d \in \mathcal{D}, k \in \mathcal{K} \backslash K^{\max } \\
& F_{t, d, 0, K^{\max }}=F_{t-1, d, 1, K^{\max }}+\widetilde{F}_{t, d, 0, K^{\max }}, \quad \forall d \in \mathcal{D} \\
& G_{t, d, 0, K^{\max }}=G_{t-1, d, 1, K^{\max }}+\widetilde{G}_{t, d, 0, K^{\max }}, \quad \forall d \in \mathcal{D} \\
& F_{t, d, r, k}=F_{t-1, d, r+1, k}+\widetilde{F}_{t, d, r, k}, \quad \forall d \in \mathcal{D}, r \in \mathcal{R} \backslash\left\{0, R^{\max }\right\}, k \in \mathcal{K} \\
& G_{t, d, r, k}=G_{t-1, d, r+1, k}+\widetilde{G}_{t, d, r, k}, \quad \forall d \in \mathcal{D}, r \in \mathcal{R} \backslash\left\{0, R^{\max }\right\}, k \in \mathcal{K} \\
& F_{t, d, R^{\max }, k}=\widetilde{F}_{t, d, R^{\max }, k}, \quad \forall d \in \mathcal{D}, k \in \mathcal{K} \\
& G_{t, d, R^{\max }, k}=\widetilde{G}_{t, d, R^{\max , k}}, \quad \forall d \in \mathcal{D}, k \in \mathcal{K}
\end{aligned}
$$

Using the transition function $S^{\mathrm{M}}$, we can rewrite (2.5) in terms of the $W_{t+1}$ as shown in (2.8). In (2.8), the only stochastic variable at stage $t$ is $W_{t}$. Note that the amount of realizations of the exogenous information $W_{t}$ is discrete and finite. We denote the set of all these realizations with $\Omega$, i.e., $W_{t} \in \Omega, \forall t \in \mathcal{T}$. For each realization $\omega \in \Omega$, there is an associated probability $p_{\omega}^{\Omega}$. Using these probabilities, the expectation in (2.8) can be rewritten, as seen in (2.9).

$$
\begin{gathered}
V_{t}\left(S_{t}\right)=\min _{x_{t} \in \mathcal{X}_{t}}\left(C\left(S_{t}, x_{t}\right)+\mathbb{E}\left\{V_{t+1}\left(S^{\mathrm{M}}\left(S_{t}, x_{t}, W_{t+1}\right)\right)\right\}\right), \forall S_{t} \in \mathcal{S} \\
V_{t}\left(S_{t}\right)=\min _{x_{t} \in \mathcal{X}_{t}}\left(C\left(S_{t}, x_{t}\right)+\sum_{\omega \in \Omega}\left(p_{\omega}^{\Omega} \cdot V_{t+1}\left(S^{\mathrm{M}}\left(S_{t}, x_{t}, \omega\right)\right)\right)\right), \forall S_{t} \in \mathcal{S}
\end{gathered}
$$

The probability $p_{\omega}^{\Omega}$ depends in three aspects of the realization $\omega \in \Omega$. First, it depends on the total number of delivery and pickup freights arriving, denoted by $f$ and $g$ respectively. Second, it depends on the probability that $\widetilde{F}_{d, r, k}^{\omega}$ delivery freights and $\widetilde{G}_{d, r, k}^{\omega}$ pickup freights will have destination $d$, release-day $r$ and time-window length $k$. Third, it depends on a multinomial coefficient $\beta$ [82] that counts the ways of assigning the total number of arriving delivery freights $f$ and pickup freights $g$ to each variable $\widetilde{F}_{d, r, k}^{\omega}$ and $\widetilde{G}_{d, r, k}^{\omega}$, respectively. This coefficient is necessary since the order in which freights arrive does not matter and freight characteristics are allowed to "repeat". With these three aspects, the probability $p_{\omega}^{\Omega}$ can be computed using (2.10).

$$
\begin{gathered}
p_{\omega}^{\Omega}=\beta \cdot p_{f}^{\mathrm{F}} p_{g}^{\mathrm{G}} \cdot \prod_{d \in \mathcal{D}, r \in \mathcal{R}, k \in \mathcal{K}}\left(\left(p_{d}^{\mathrm{D}, \mathrm{F}} p_{r}^{\mathrm{R}, \mathrm{F}} p_{k}^{\mathrm{K}, \mathrm{F}}\right)^{\widetilde{F}_{d, r, k}^{\omega}}\left(p_{d}^{\mathrm{D}, \mathrm{G}} p_{r}^{\mathrm{R}, \mathrm{G}} p_{k}^{\mathrm{K}, \mathrm{G}}\right)^{\widetilde{G}_{d, r, k}^{\omega}}\right) \\
\omega=\left[\left(\widetilde{F}_{d, r, k}^{\omega}, \widetilde{G}_{d, r, k}^{\omega}\right)\right]_{\forall d \in \mathcal{D}, r \in \mathcal{R}, k \in \mathcal{K}}
\end{gathered}
$$




$$
\begin{gathered}
f=\sum_{d \in \mathcal{D}, r \in \mathcal{R}, k \in \mathcal{K}} \widetilde{F}_{d, r, k}^{\omega} \quad, \quad g=\sum_{d \in \mathcal{D}, r \in \mathcal{R}, k \in \mathcal{K}} \widetilde{G}_{d, r, k}^{\omega} \\
\beta=\frac{f !}{\prod_{d \in \mathcal{D}, r \in \mathcal{R}, k \in \mathcal{K}}\left(\widetilde{F}_{d, r, k}^{\omega} !\right)} \cdot \frac{g !}{\prod_{d \in \mathcal{D}, r \in \mathcal{R}, k \in \mathcal{K}}\left(\widetilde{G}_{d, r, k}^{\omega} !\right)}
\end{gathered}
$$

In the MDP model above, the formulation for the states, decisions, exogenous information, transition, and costs of the round-trip problem can be separated into a delivery and a pickup part considering freights that are described with the same names (i.e., variables and parameters that contain an $F$ and a $G$ as a superscript, respectively). If we consider only the delivery part (although considering the pickup part would be the same since the two parts are formulation-wise identical to each other), the result is a single-trip rather than a round-trip. In the case of the LSP that motivated this problem, a single-trip would correspond to an external barge that is hired for half of the trip, meaning that once it delivers (or picks) the freight, the LSP is no longer responsible for the scheduling. We come back to this simplified problem in Section 2.6.

The optimal costs and the optimal policy for the long-haul round-trip (and single-trip) scheduling problem can be found solving (2.9), for instance, using dynamic programming and the probabilities from (2.10). However, the computational effort increases faster than exponential with increasing size of the problem instance. In the following, we elaborate on the dimensionality issues of our model.

\subsubsection{Dimensionality Issues}

The optimality equations in (2.9) suffer from what Powell [76] calls the "three curses of dimensionality". The first curse comes from the set of all possible realizations of the exogenous information $\Omega$. For each possible decision $x_{t} \in \mathcal{X}$, the calculation of the expectation requires the next-stage value of $|\Omega|$ states. The second issue comes from evaluating all possible decisions. At each state, finding the decision that minimizes the sum of the current-day and expected future costs involves the evaluation of all possible combinations of the freights that are released. The third, and most difficult of all dimensionality issues, comes from the set of all possible states $\mathcal{S}$. In our model, the number of possible states increases faster than exponential with increasing domain of destinations, release-days, and time-window lengths. In addition, the number of release-days $|\mathcal{R}|$ determines the extent to which freights can "accumulate". To provide the reader with a measurable idea on these issues, we elaborate on the first curse of dimensionality in Appendix 2-A. Due to these dimensionality issues, an exact solution to (2.9) using backward dynamic programming, for example, is only feasible in small problem instances. Nevertheless, the model provides the foundation for our ADP approach, which can be applied to realistic instance sizes. 


\subsection{Solution Algorithm}

We propose a solution algorithm based on Approximate Dynamic Programming (ADP). ADP is a framework that contains several methods for tackling the curses of dimensionality in an MDP. The general idea of ADP is to modify Bellman's equations with a series of components and algorithmic manipulations in order to approximate their solution, and thus the optimal policy. In this section, we elaborate on the components and algorithmic manipulations we use, as shown in Algorithm 1. First, we introduce the concepts of post-decision state and forward dynamic programming, which tackle the first and third dimensionality issue mentioned in Section 2.3.3. Second, we introduce the concept of basis functions as an approximation of the value of the post-decision states. Finally, we describe a way of tackling the second dimensionality issue of finding the optimal decision for a single stage.

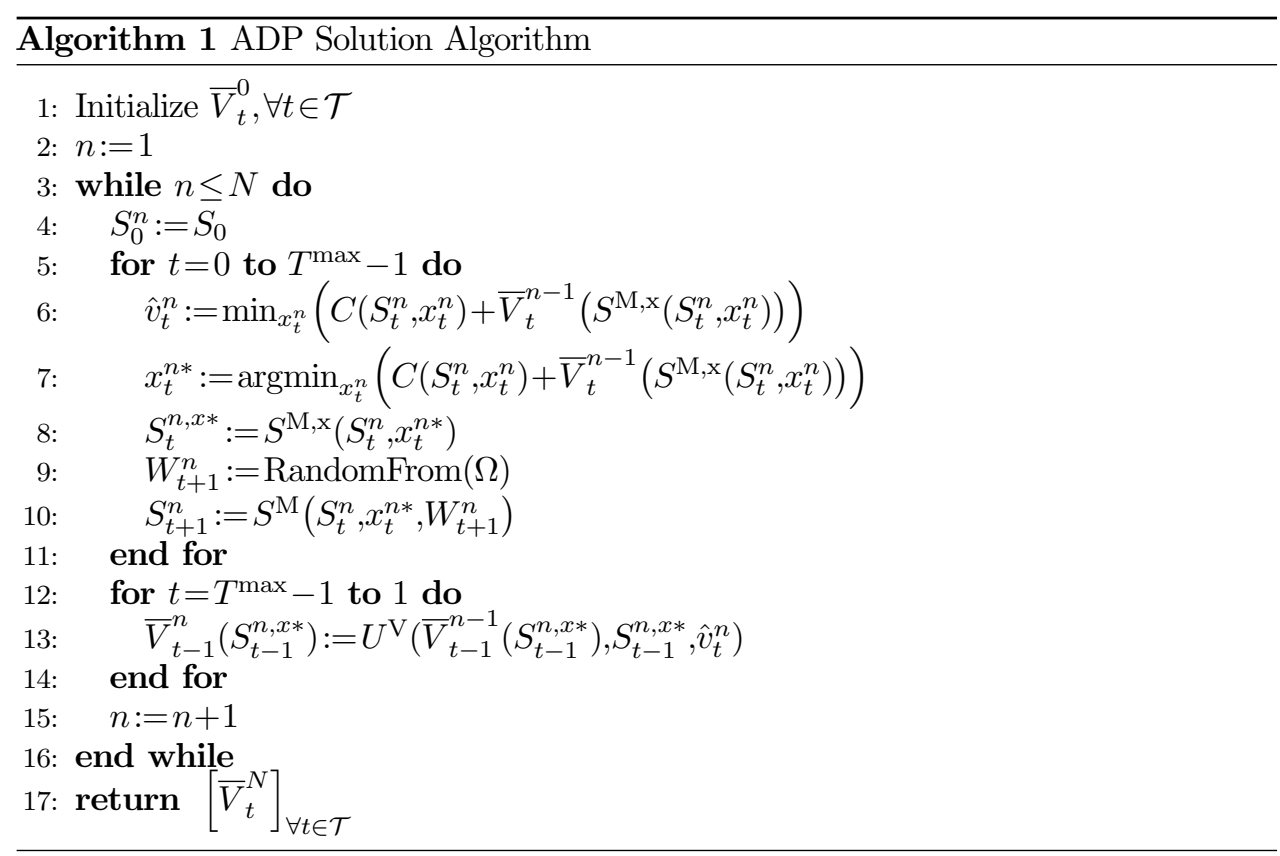

\subsubsection{Post-decision State and Forward Dynamic Programming}

To tackle the large set of realizations of the exogenous information $\Omega$, we introduce two new components into the model: (i) a post-decision state $S_{t}^{n, x}$, and (ii) an approximated next-stage cost $\bar{V}_{t}^{n}\left(S_{t}^{n, x}\right)$. The post-decision state is the state of the system directly after a decision $x_{t}^{n}$ has been made but before the exogenous information $W_{t}^{n}$ becomes known, at iteration $n=1,2, \ldots, N$ of the algorithm. The approximated next-stage cost $\bar{V}_{t}^{n}\left(S_{t}^{n, x}\right)$ serves 
as an estimated measurement for the next-stage costs (i.e., $\left.\bar{V}_{t}^{n}\left(S_{t}^{n, x}\right) \approx \mathbb{E}\left\{V_{t+1}\left(S_{t+1}\right)\right\}\right)$. We elaborate on this measurement later on. For now, we focus on the post-decision state. In a similar way to the freight variables of a state, the post-decision freight variables $F_{t, d, r, k}^{n, x}$ and $G_{t, d, r, k}^{n, x}$ form the post-decision state $S_{t}^{n, x}$, as seen in (2.11). Note that these components are all indexed with a superscript $n$, which denotes the iteration $n$ they correspond to.

$$
S_{t}^{n, x}=\left[\left(F_{t, d, r, k}^{n, x}, G_{t, d, r, k}^{n, x}\right)\right]_{\forall d \in \mathcal{D}, r \in \mathcal{R}, k \in \mathcal{K}}
$$

To define a post-decision state $S_{t}^{n, x}$, we define a function $S^{\mathrm{M}, \mathrm{x}}$ that relates the postdecision freight variables $S_{t}^{n, x}$ with the state $S_{t}^{n}$ and decision $x_{t}^{n}$, as shown in (2.12). The workings of this function are similar to the transition function $S^{\mathrm{M}}$ defined in (2.7), leaving out the exogenous information $W_{t+1}^{n}$.

$$
\begin{gathered}
S_{t}^{n, x}=S^{\mathrm{M}, \mathrm{x}}\left(S_{t}^{n}, x_{t}^{n}\right), \forall t \in \mathcal{T} \\
\text { where } \\
F_{t, d, 0, k}^{n, x}=F_{t, d, 0, k+1}^{n}-x_{t, d, k+1}^{n, \mathrm{~F}}+F_{t, d, 1, k}^{n}, \quad \forall d \in \mathcal{D}, k \in \mathcal{K} \backslash K^{\max } \\
G_{t, d, 0, k}^{n, x}=G_{t, d, 0, k+1}^{n}-x_{t, d, k+1}^{n, \mathrm{G}}+G_{t, d, 1, k}^{n}, \quad \forall d \in \mathcal{D}, k \in \mathcal{K} \backslash K^{\max } \\
F_{t, d, 0, K^{\max }}^{n, x} F_{t, d, 1, K^{\max }}^{n}, \quad \forall d \in \mathcal{D} \\
G_{t, d, 0, K^{\max }}^{n, x}=G_{t, d, 1, K^{\max }}^{n}, \quad \forall d \in \mathcal{D} \\
F_{t, d, r, k}^{n, x}=F_{t, d, r+1, k}^{n}, \quad \forall d \in \mathcal{D}, r \in \mathcal{R} \backslash\left\{1, R^{\max }\right\}, k \in \mathcal{K} \\
G_{t, d, r, k}^{n, x}=G_{t, d, r+1, k}^{n}, \quad \forall d \in \mathcal{D}, r \in \mathcal{R} \backslash\left\{1, R^{\max }\right\}, k \in \mathcal{K}
\end{gathered}
$$

To tackle the large state space $\mathcal{S}$, we use the algorithmic manipulation of "forward dynamic programming". In contrast to backward dynamic programming, forward dynamic programming starts at the first stage and, at each stage, solves an "optimality" equation for only one state, as seen in (2.13). This equation follows the same reasoning as the Bellman's equation from (2.5), with two differences: (i) the next-stage costs are approximated, and (ii) each feasible decision $x_{t}^{n}$ has only one corresponding post-decision state.

$$
\begin{aligned}
\hat{v}_{t}^{n} & =\min _{x_{t}^{n} \in \mathcal{X}_{t}}\left(C\left(S_{t}^{n}, x_{t}^{n}\right)+\bar{V}_{t}^{n-1}\left(S_{t}^{n, x}\right)\right) \\
& =\min _{x_{t}^{n} \in \mathcal{X}_{t}}\left(C\left(S_{t}^{n}, x_{t}^{n}\right)+\bar{V}_{t}^{n-1}\left(S^{M, x}\left(S_{t}^{n}, x_{t}^{n}\right)\right)\right)
\end{aligned}
$$

To advance in time, a random realization $W_{t+1}^{n}$ from the set of exogenous information $\Omega$ is obtained via a Monte Carlo simulation. Subsequently, the transition function $S^{\mathrm{M}}$ of the MDP model, defined in (2.7), is used. Although the simulation step introduces variability in the algorithm, it also represents the stochastic nature of the problem and can be used to improve the approximation iteratively. After processing the entire horizon, the approximated next-stage cost $\bar{V}_{t}^{n}\left(S_{t}^{n, x}\right)$ is updated retrospectively. In the following section, we explain how the approximation works and how it is updated in every iteration. 


\subsubsection{Basis Functions and Non-stationary Least-Squares Update Function}

The approximated next-stage $\operatorname{cost} \bar{V}_{t}^{n}\left(S_{t}^{n, x}\right)$ represents the future costs after stage $t$, estimated at iteration $n$. To build a good approximation, we use the frequently used approximation strategy of "basis functions". Basis functions are quantitative characteristics, or features, of a post-decision state, which explain, to some extent, the next-stage costs. In our problem, features such as the number of urgent freights, the number of released freights that are not urgent, and the number of freights that have not been released for transport, can explain part of the value of a post-decision state. Basis functions are then designed to quantify the impact of the features they represent on the value function. In the remainder of this chapter and the next one, we refer to basis function and feature indistinctly. We denote a basis function as $\phi_{a}\left(S_{t}^{n, x}\right)$, where $a$ belongs to the set of features $\mathcal{A}$. The approximated next-stage cost of a post-decision stage $\bar{V}_{t}^{n}\left(S_{t}^{n, x}\right)$ can be expressed as a weighted sum of all basis functions, as shown in (2.14), where $\theta_{a, t}^{n} \in \mathbb{R}$ is the weight of each basis function $a \in \mathcal{A}$ at stage $t$ and iteration $n$. Note that (2.14) is a linear approximation, as it is linear in its parameters. The basis functions themselves can be nonlinear [77]. Using matrix notation, we define $\phi\left(S_{t}^{x, n}\right)=\left[\phi_{1, t}\left(S_{t}^{x, n}\right), \ldots, \phi_{\left|\mathcal{A}_{t}\right|, t}\left(S_{t}^{x, n}\right)\right]^{T}$ as the column vector of basis functions, and $\theta_{t}^{n}=\left[\theta_{1, t}^{n}, \ldots, \theta_{|\mathcal{A}|, t}^{n}\right]^{T}$ as the column vector of weights at iteration $n$ and stage $t$. We will use these vectors to describe the updating procedure later on.

$$
\bar{V}_{t}^{n}\left(S_{t}^{x, n}\right)=\sum_{a \in \mathcal{A}} \theta_{a, t}^{n} \phi_{a}\left(S_{t}^{x, n}\right)=\phi\left(S_{t}^{x, n}\right)^{T} \theta_{t}^{n}
$$

Naturally, with each iteration there are more observations of the stochastic costs of the problem. Since in every iteration and every stage, a Monte Carlo simulation of the exogenous information is performed and a decision is made, the costs of the newly seen state can be used to improve our knowledge of the next-stage costs. However, due to the time nature of the post-decision state, costs have to be updated retrospectively. We define a function $U^{\mathrm{V}}$ to denote the process that updates the approximated costs $\bar{V}_{t-1}^{n}\left(S_{t-1}^{n, x}\right)$ at iteration $n$, using (i) the approximated costs, from the previous iteration, of the post-decision state of the current iteration and of the previous stage $\bar{V}_{t-1}^{n-1}\left(S_{t-1}^{n, x}\right)$, (ii) the post-decision state of the current iteration and the previous stage itself $\left(S_{t-1}^{n, x}\right)$, and (iii) the solution to the forward optimality equation $\hat{v}_{t}^{n}$ corresponding to the current iteration and of the current stage, as seen in (2.15).

$$
\bar{V}_{t-1}^{n}\left(S_{t-1}^{n, x}\right)=U^{\mathrm{V}}\left(\bar{V}_{t-1}^{n-1}\left(S_{t-1}^{n, x}\right), S_{t-1}^{n, x}, \hat{v}_{t}^{n}\right)
$$

The logic behind the retrospective update is as follows. At stage $t$ of iteration $n$, the system has moved from $S_{t-1}^{n}$ to $S_{t}^{n}$. The optimal costs $\hat{v}_{t}^{n}$ of $S_{t}^{n}$ are the "realized" next-stage costs of the previous-stage post-decision state that the algorithm estimated in the previous iteration. In other words, the approximated next-stage cost that was calculated at the $t-1$, using the previous iteration $n-1$ estimate, has now been observed at $t$ in iteration $n$.

The use of basis functions and weights for approximating the value function $\bar{V}_{t}^{n}\left(S_{t}^{x, n}\right)$ is comparable to the use of regression models for fitting data to a (linear) function. In 
that sense, the independent variables of the regression model would be the features of the post-decision state and the dependent variable would be the value of the post-decision state. However, in contrast to regression models, the data in our ADP approach is generated iteratively inside an algorithm and not all at once. Therefore, the updating process $U^{\mathrm{V}}$ for the approximating function in (2.15) cannot be based only on solving systems of equations as in traditional regression models.

Several methods are available to "fine-tune" the weights $\theta_{a}^{n}$ for each feature $a \in \mathcal{A}$ after each iteration. An effective approach is the recursive least squares method, which is a technique to compute the solution to a linear least squares problem [77]. This method updates each weight based on the observed error $\left(\bar{V}_{t-1}^{n-1}\left(S_{t-1}^{n, x}\right)-\hat{v}_{t}^{n}\right)$ and the value of its corresponding basis function $\phi_{a}\left(S_{t}^{n, x}\right)$. Two types of recursive least squares methods are available. The least squares method for nonstationary data provides the opportunity to put increased weight on more recent observations, whereas the least squares method for stationary data puts equal weight on each observation. For the purpose of learning the weights within an ADP algorithm, the recursive least squares method for nonstationary data is more appropriate. The method for updating the value function approximations with the recursive least squares method for nonstationary data is explained in detail in [77]. Nevertheless, the equations used in this method are given in (2.16).

$$
\begin{gathered}
\theta_{a}^{n}=\theta_{a}^{n-1}-H_{n} \phi_{a}\left(S_{t}^{x, n}\right)\left(\bar{V}_{t-1}^{n-1}\left(S_{t-1}^{x, n}\right)-\widehat{v}_{t}^{n}\right) \\
\text { where } \\
H^{n}=\frac{1}{\gamma^{n}} B^{n-1} \\
B^{n}=\frac{1}{\alpha^{n}}\left(B^{n-1}-\frac{1}{\gamma^{n}}\left(B^{n-1} \phi\left(S_{t}^{x, n}\right)\left(\phi\left(S_{t}^{x, n}\right)\right)^{T} B^{n-1}\right)\right) \\
\gamma^{n}=\alpha^{n}+\phi\left(S_{t}^{x, n}\right)^{T} B^{n-1} \phi\left(S_{t}^{x, n}\right) .
\end{gathered}
$$

The weights $\theta_{a}^{n}$, for all $a \in \mathcal{A}$, are updated each iteration $n$ as shown in (2.16). In (2.16), $H^{n}$ and $B^{n}$ are $|\mathcal{A}| \times|\mathcal{A}|$ optimization matrices. $B^{n}$ is initialized by using $B^{0}=\epsilon I$, where $I$ is the identity matrix and $\epsilon$ is a small constant. This initialization works well when the number of observations is large [77]. The parameter $\alpha^{n}$ determines the weight on prior observations of the value. Setting $\alpha^{n}$ equal to 1 for each $n$ would set equal weight on each observation, and implies that the least squares method for stationary data is being used. Setting $\alpha^{n}$ to values between 0 and 1 decreases the weight on prior observations (lower $\alpha^{n}$ means lower weight on). For our problem, $\alpha^{n}=1-0.5 / n$ is a function that works well in our experiments. Solving (2.16) takes places in a "double-pass" fashion in lines 12 to 14, which further reduces the approximation bias. For more information on the double-pass logic, see Powell [77].

Finally, the decision space $\mathcal{X}_{t}$ is relatively small for the problem settings we test in this chapter. Our goal in this chapter is to analyze the ADP workings on small problem instances that we can solve exactly, i.e., get the optimal solution from the MDP model. Nevertheless, for larger problem instances, as the ones considered in Chapter 3, enumerating all possible 
decisions can be computationally difficult. To tackle this second dimensionality issue of finding the optimal decision for a single stage, we formulate a Mixed-Integer Linear Program (MILP) for the single-stage decision problem, as seen in Appendix 2-B. In the following section, we analyze the performance of the ADP approach compared to the optimal solution of the MDP model, for small test instances.

\subsection{Experimental Setup}

In this section, we describe the experimental settings we use to study the accuracy of our ADP approach (i.e., optimality gap) and to study the design and evaluation challenges that arise when choosing the best set of features for ADP. Due to the dimensionality issues of the MDP model, solving the Bellman equations in (2.9) using backwards dynamic programming is only possible for small instances of the problem. We create six small-sized instances: $I_{1}^{S}$ through $\mathrm{I}_{6}^{S}$, which are considerably smaller than the network of the Dutch LSP, in terms of number of freights and destinations, but have similar relations of probability distributions. The probability distributions for these six instances are shown in Table 2.1. Furthermore, we consider a time horizon of a working week $T^{\max }=5$ and a vehicle capacity of $Q=2$ for all six instances. The ranges for the costs are the same for all six instances, and are defined as follows: $C_{\mathcal{D}^{\prime}}=[250,1000], B_{d}=[50,100]$, and $A_{d}=[500,1000]$. Remind that $C_{\mathcal{D}^{\prime}}$ is the cost of visiting a subset of destinations with the high-capacity mode (i.e., transport cost), $B_{d}$ is the cost per freight consolidated in the high-capacity mode, to a given destination (i.e., handling, loading, unloading cost), and $A_{d}$ is the cost per freight transported using the alternative mode, to a given destination (i.e., transport and handling cost). This cost setup is based upon three cost considerations. First, there are costs only if the high-capacity mode departs or the alternative transport mode is used. Second, the high-capacity mode costs depend predominantly on the subset of destinations visited. This means that the costs for transporting an additional container to (or from) a destination already scheduled to be visited are small compared to visiting an additional destination. Third, using the alternative mode, for the same number of freights that a high-capacity mode can carry, is more expensive. This reflects the economies of scale achieved through consolidation in high-capacity modes.

The reasoning behind varying the probability distributions in Instances $\mathrm{I}_{1}^{S}$ to $\mathrm{I}_{6}^{S}$ is to test the optimality gap in different network characteristics. For example, $\mathrm{I}_{1}^{S}$ represents a "balanced" network, where the variability in delivery freight is the same as the one in pickup freights, whereas $I_{2}^{S}$ represents an "unbalanced" network where this variability differs. Furthermore, Instances $\mathrm{I}_{3}^{S}$ and $\mathrm{I}_{4}^{S}$ represent balanced networks with the freights having a short time-window and a long time-window, respectively. The shorter the time-window, the less possibilities to postpone freights. Finally, $\mathrm{I}_{5}^{S}$ and $\mathrm{I}_{6}^{S}$ follow the same idea of testing opposite time-window distributions, but for unbalanced networks.

Designing a set of features of a post-decision state that properly captures future costs within an ADP algorithm is both a science and an art. With scientific approaches such as factor analysis and regression analysis, one can conjecture how "good" a feature is. However, designing features requires creativity about the causes of costs. We build three different sets of features based on a common "job" description used in transport problems: 
Chapter 2. Long-haul Round-trip Transport: Solution Design

Table 2.1: Input parameters of the numerical experiments

\begin{tabular}{|c|c|c|c|c|c|c|}
\hline \multirow{2}{*}{ Parameter } & \multicolumn{6}{|c|}{ Instance } \\
\hline & $\mathrm{I}_{1}^{S}$ & $\mathrm{I}_{2}^{S}$ & $\mathrm{I}_{3}^{S}$ & $\mathrm{I}_{4}^{S}$ & $\mathrm{I}_{5}^{S}$ & $\mathrm{I}_{6}^{S}$ \\
\hline $\mathcal{D}$ & $\{1,2,3\}$ & $\{1,2,3\}$ & $\{1,2,3\}$ & $\{1,2,3\}$ & $\{1,2,3\}$ & $\{1,2,3\}$ \\
\hline $\begin{array}{l}p_{d}^{\mathrm{D}, \mathrm{F}} \\
p_{d}^{\mathrm{D}, \mathrm{G}}\end{array}$ & $\begin{array}{l}\left\{\frac{1}{10}, \frac{8}{10}, \frac{1}{10}\right\} \\
\left\{\frac{1}{10}, \frac{8}{10}, \frac{1}{10}\right\}\end{array}$ & $\begin{array}{c}\left\{\frac{1}{3}, \frac{1}{3}, \frac{1}{3}\right\} \\
\left\{\frac{1}{10}, \frac{8}{10}, \frac{1}{10}\right\}\end{array}$ & $\begin{array}{l}\left\{\frac{1}{10}, \frac{8}{10}, \frac{1}{10}\right\} \\
\left\{\frac{1}{10}, \frac{8}{10}, \frac{1}{10}\right\}\end{array}$ & $\begin{array}{l}\left\{\frac{1}{10}, \frac{8}{10}, \frac{1}{10}\right\} \\
\left\{\frac{1}{10}, \frac{8}{10}, \frac{1}{10}\right\}\end{array}$ & $\begin{array}{c}\left\{\frac{1}{3}, \frac{1}{3}, \frac{1}{3}\right\} \\
\left\{\frac{1}{10}, \frac{8}{10}, \frac{1}{10}\right\}\end{array}$ & $\begin{array}{c}\left\{\frac{1}{3}, \frac{1}{3}, \frac{1}{3}\right\} \\
\left\{\frac{1}{10}, \frac{8}{10}, \frac{1}{10}\right\}\end{array}$ \\
\hline $\mathcal{R}$ & $\{0\}$ & $\{0\}$ & $\{0\}$ & $\{0\}$ & $\{0\}$ & $\{0\}$ \\
\hline$p_{r}^{\mathrm{R}, \mathrm{F}}$ & $\{1\}$ & $\{1\}$ & $\{1\}$ & $\{1\}$ & $\{1\}$ & $\{1\}$ \\
\hline$p_{r}^{\mathrm{R}, \mathrm{G}}$ & $\{1\}$ & $\{1\}$ & $\{1\}$ & $\{1\}$ & $\{1\}$ & $\{1\}$ \\
\hline $\mathcal{K}$ & $\{0,1,2\}$ & $\{0,1,2\}$ & $\{0,1,2\}$ & $\{0,1,2\}$ & $\{0,1,2\}$ & $\{0,1,2\}$ \\
\hline $\begin{array}{l}p_{k}^{\mathrm{K}, \mathrm{F}} \\
p_{k}^{\mathrm{K}, \mathrm{G}}\end{array}$ & $\begin{array}{l}\left\{\frac{2}{10}, \frac{3}{10}, \frac{5}{10}\right\} \\
\left\{\frac{2}{10}, \frac{3}{10}, \frac{5}{10}\right\}\end{array}$ & $\begin{array}{c}\left\{\frac{1}{3}, \frac{1}{3}, \frac{1}{3}\right\} \\
\left\{\frac{2}{10}, \frac{3}{10}, \frac{5}{10}\right\}\end{array}$ & $\begin{array}{l}\left\{\frac{8}{10}, \frac{1}{10}, \frac{1}{10}\right\} \\
\left\{\frac{8}{10}, \frac{1}{10}, \frac{1}{10}\right\}\end{array}$ & $\begin{array}{l}\left\{\frac{1}{10}, \frac{1}{10}, \frac{8}{10}\right\} \\
\left\{\frac{1}{10}, \frac{1}{10}, \frac{8}{10}\right\}\end{array}$ & $\begin{array}{l}\left\{\frac{8}{10}, \frac{1}{10}, \frac{1}{10}\right\} \\
\left\{\frac{8}{10}, \frac{1}{10}, \frac{1}{10}\right\}\end{array}$ & $\begin{array}{l}\left\{\frac{1}{10}, \frac{1}{10}, \frac{8}{10}\right\} \\
\left\{\frac{1}{10}, \frac{1}{10}, \frac{8}{10}\right\}\end{array}$ \\
\hline $\mathcal{F}=\mathcal{G}$ & $\{1\}$ & $\{1\}$ & $\{1\}$ & $\{1\}$ & $\{1\}$ & $\{1\}$ \\
\hline$p_{f}^{\mathrm{F}}$ & $\{1\}$ & $\{1\}$ & $\{1\}$ & $\{1\}$ & $\{1\}$ & $\{1\}$ \\
\hline$p_{f}^{G}$ & $\{1\}$ & $\{1\}$ & $\{1\}$ & $\{1\}$ & $\{1\}$ & $\{1\}$ \\
\hline
\end{tabular}

MustGo, MayGo, and Future jobs. In our case, MustGo freights are those released freights whose due-day is immediate. MayGo freights are those released freights whose due-day is not immediate. Future freights are those that have not yet been released. We use the MustGo, MayGo and Future adjectives in destinations as well, with an analogous meaning. In Table 2.2 we show the three sets of features, which we name Features 1, 2, and 3. All feature types in this table are related to the freights of a post-decision state. The symbol "•" denotes a set containing the corresponding feature type. All feature types are numerical, and either indicate (i.e., 1 if yes, 0 if no), count $(1,2, \ldots)$, number (add), or multiply (i.e., product between two numbers) the different type of freights and destinations. Between parentheses we show the number of basis functions of each feature type. For example, there is one post-decision state variable per destination, per time-window length, both for the delivery and the pickup, resulting in a total of $3^{*} 3^{*} 2=18$ post-decision state variables.

To measure the optimality gap of the ADP approach in combination with each set of features, in each small instance, we use a two-step methodology. First, we apply the ADP algorithm to each of the 19,321 states of the instance. In our experiments, 500 iterations are enough for the algorithm to converge to a constant estimate of the costs of each initial state, within a reasonable computational time. A larger number of iterations would not guarantee a better performance since the state space is too large to measure it in its entirety within reasonable time, and the performance is also influenced by the chosen approximation 
Table 2.2: Three sets of features for the long-haul round-trip costs

\begin{tabular}{|c|c|c|c|}
\hline Feature type & Features 1 & Features 2 & Features 3 \\
\hline All post-decision state variables (18) & • & • & - \\
\hline All post-decision state variables squared (18) & • & - & - \\
\hline Count of MustGo destinations (1) & • & $\bullet$ & • \\
\hline Number of MustGo freights (1) & - & - & • \\
\hline Product of MustGo destinations and MustGo freights (1) & $\bullet$ & - & - \\
\hline Count of MayGo destinations (1) & • & - & • \\
\hline Number of MayGo freights (1) & $\bullet$ & $\bullet$ & $\bullet$ \\
\hline Product of MayGo destinations and MayGo freights (1) & $\bullet$ & - & - \\
\hline Count of Future destinations (1) & $\bullet$ & $\bullet$ & • \\
\hline Number of Future freights (1) & $\bullet$ & $\bullet$ & $\bullet$ \\
\hline Product of Future destinations and Future freights (1) & • & - & - \\
\hline Indicator MustGo freights per destination (3) & - & - & - \\
\hline Indicator MayGo freights per destination (3) & - & - & - \\
\hline Indicator Future freights per destination (3) & - & - & - \\
\hline Number of all freights (1) & • & • & • \\
\hline Constant (1) & $\bullet$ & • & • \\
\hline
\end{tabular}

function (i.e., set of features) and the updating mechanism. Second, for each state, we simulate the use of the policy resulting from the ADP algorithm on 500 replications of the time horizon. We store the average costs of this simulation, for each state, and calculate the percentage difference between these average performance costs and the optimal expected costs. Finally, the optimality gap is measured as the average percentage difference over all states, for each set of features and each small instance.

Besides the experiments just described, we carry out experiments with a simpler version of the long-haul round-trip scheduling problem: the single-trip problem introduced at the end of Section 2.3.2. A single-trip, as opposed to a round-trip, considers only the delivery freights that need to be transported from the single origin to the destinations. We follow the same methodology described in the previous paragraph to allow us to compare the design challenges of ADP between the single-trip and round-trip versions of our problem. Furthermore, we take a closer look at two states of Instance $\mathrm{I}_{1}^{S}$ of the single-trip to show the different design challenges arising when applying the ADP algorithm with basis functions to different initial states. The two states of Instance $\mathrm{I}_{1}^{S}$ are tested with an increase in arriving freights: either one or two freights arrive each period (i.e., $\mathcal{F}=\{1,2\}$ ), with probability $p_{f}^{F}=(0.8,0.2)$ for $f \in \mathcal{F}$. The first state, referred to as "State 1" has only one freight for destination 2 with a time-window length of 2 (i.e. $F_{0,2,0,2}=1$ ). The second state, referred to as "State 2", has a total of six freights: one urgent freight for destination 2 and 3 , three freights for destination 2 with time-window length 1 , and one freight for destination 2 with time-window length 2 (i.e., $F_{0,2,0,0}=F_{0,3,0,0}=1, F_{0,2,0,1}=3, F_{0,2,0,2}=1$ ). 


\subsection{Numerical Results}

In this section, we present the results of our experiments. ADP performance refers to the result of the simulation of the policy dictated by the feature weights learned by the ADP algorithm. We test the performance of the Features from Table 2.2, over the entire state space. Furthermore, for each instance we solve the MDP model and obtain the optimal costs for all states. We show the optimality gap as a percentage and the Mean Square Error (MSE), which is a measure for the spread of the error among all states of an instance, in Table 2.3. In Table 2.4, we show the average costs for each instance, over all states, for the MDP model (i.e., optimal costs) and for the three Features, and their computational times, in seconds on a personal computer. The time for the VFAs corresponds to the average time it takes the ADP algorithm to run the 500 iterations and learn the weights in (2.14). Remind that ADP starts with an initial state but generalizes the future costs for all possible states within the horizon. In the following, we discuss these results in detail.

Table 2.3: Optimality gap of the Features in instances $I_{1}^{S}$ to $I_{6}^{S}$

\begin{tabular}{lcccccc}
\hline \multirow{2}{*}{ Instance } & \multicolumn{2}{c}{ Features 1 } & \multicolumn{2}{c}{ Features 2 } & \multicolumn{2}{c}{ Features 3 } \\
& Avg. & MSE & Avg. & MSE & Avg. & MSE \\
\hline $\mathrm{I}_{1}^{S}$ & $4.7 \%$ & 34203 & $4.1 \%$ & 27253 & $4.0 \%$ & 25213 \\
$\mathrm{I}_{2}^{S}$ & $6.2 \%$ & 57065 & $6.2 \%$ & 54110 & $5.9 \%$ & 51032 \\
$\mathrm{I}_{3}^{S}$ & $2.1 \%$ & 16196 & $1.9 \%$ & 14409 & $2.0 \%$ & 14824 \\
$\mathrm{I}_{4}^{S}$ & $3.3 \%$ & 23622 & $3.7 \%$ & 24878 & $3.5 \%$ & 24148 \\
$\mathrm{I}_{5}^{S}$ & $1.5 \%$ & 11895 & $1.5 \%$ & 10000 & $1.3 \%$ & 9405 \\
$\mathrm{I}_{6}^{S}$ & $7.1 \%$ & 66958 & $7.0 \%$ & 67880 & $6.5 \%$ & 60010 \\
\hline
\end{tabular}

Table 2.4: Costs and times of the MDP model and the Features in instances $I_{1}^{S}$ to $I_{6}^{S}$

\begin{tabular}{lcccccccc}
\hline \multirow{2}{*}{ Instance } & \multicolumn{2}{c}{ MDP } & \multicolumn{2}{c}{ Features 1 } & \multicolumn{2}{c}{ Features 2 } & \multicolumn{2}{c}{ Features 3 } \\
& Avg. & Time & Avg. & Time & Avg. & Time & Avg. & Time \\
\hline $\mathrm{I}_{1}^{S}$ & 3245 & 823.1 & 3396 & 3.1 & 3376 & 4.7 & 3372 & 2.5 \\
$\mathrm{I}_{2}^{S}$ & 3447 & 820.3 & 3657 & 3.0 & 3657 & 4.7 & 3649 & 2.4 \\
$\mathrm{I}_{3}^{S}$ & 4228 & 795.7 & 4317 & 3.0 & 4309 & 4.8 & 4312 & 2.4 \\
$\mathrm{I}_{4}^{S}$ & 2921 & 843.2 & 3017 & 2.4 & 3028 & 4.7 & 3024 & 1.9 \\
$\mathrm{I}_{5}^{S}$ & 4405 & 850.1 & 4469 & 2.1 & 4469 & 4.6 & 4462 & 1.6 \\
$\mathrm{I}_{6}^{S}$ & 2975 & 843.2 & 3183 & 2.4 & 3179 & 4.9 & 3166 & 1.7 \\
\hline
\end{tabular}

We observe that there are no significant differences among the average optimality gaps of the Features. Features 1 ranges between $1.5 \%$ and $7.1 \%$ away from optimal, Features 2 between $1.5 \%$ and $7.0 \%$, and Features 3 between $1.3 \%$ and $6.5 \%$. With respect to the spread of the error within an instance, differences among the Features are more noticeable. For 
example, in Instance $I_{2}^{S}$, both Features 1 and Features 2 have the same average optimality gap, but Features 2 has a lower MSE indicating a smaller spread in error across states of the same instance. We reflect upon the difference in optimality gap among states of the same instance in Section 2.7. Finally, the most noticeable difference among the Features arise in computational time. Although all Features are significantly faster than the MDP (3s against 830s on average), Features 3 is the fastest and Features 2 the slowest. Note that 830 s is still reasonable for daily decision making, however, for realistic instances the MDP cannot be solved within a day.

Focusing on differences among instances, we observe that when freights have longer time-windows, the optimality gap of the Features is lower than when they have shorter time-windows. This result indicates that the more freights can be postponed in a network, the better our ADP algorithm can approximate the optimal policy. The lowest optimality gap is achieved in Instance $\mathrm{I}_{5}^{S}$ (1.4\% on average). This result indicates that a network that has many and complex possibilities for postponing freights, such as the "unbalanced" Instance $\mathrm{I}_{5}^{S}$ with long time-windows, has more potential for our algorithm to identify the optimal policy. We also observe that in terms of the optimality gap and MSE, Instance $\mathrm{I}_{6}^{S}$ was the most difficult for all Features. We elaborate on the difficulties of the ADP performance under different problem settings (instances) in Section 2.7.

All the previous experiments where done by simulating the use of the weights resulting from the ADP algorithm with each set of features, for of all states in the state space. However, statistical inference can be used to conjecture about the potential of different sets of features. For example, due to the linear relation between basis functions and estimated future costs (in the value function approximation of the ADP algorithm), the coefficient of determination $R^{2}$ of a linear regression is a measure that could be used to infer which is the best set of features. Computing this measure is faster than simulating the use of each set of features for all states. However, its results can mislead the designer on what performance to expect from a given set of features. We use $R^{2}$ to show the difficulties in establishing the potential of basis functions (i.e., sets of features of a state) in the design of the ADP algorithm.

To exemplify the use of $R^{2}$, we use the six instances defined in Table 2.1 with the cost $B_{d}=0$ and show six additional instances denoted by $\mathrm{I}^{S}$-st in Table 2.5. These instances correspond to the single-trip problem of Instances $\mathrm{I}_{1}^{S}$ to $\mathrm{I}_{6}^{S}$ (hence the -st suffix). The results of the optimality gap experiments, and the linear regression, are shown in Table 2.5. We observe that all single-trip instances have a higher $R^{2}$ than the round-trip ones, but half of them have worse optimality gap. This result shows that, although applying a statistical test to a simplified version of the problem can be easier computational-wise, it can also lead to a different choice of "best" set of features. Furthermore, we observe that a higher coefficient of determination does not necessarily mean a lower optimality gap, especially in the round-trip instances. This result indicates that more basis functions to capture costs does not necessarily lead to a better approximation of the optimal policy.

In addition, we can analyze the sensitivity of ADP to the cost setting $B_{d}>0$ and $B_{d}=0$ by comparing Table 2.3 and Table 2.5. We observe that all instances in Table 2.3 have a lower optimality gap and MSE than in Table 2.5. In other words, ADP is closer to optimal in the cost setting $B_{d}>0$. A reason for this is the fact that there are larger differences in costs among similar states, making it easier for ADP to identify which post-decision states are 
Table 2.5: Performance of the different Features in instances $I_{1}^{S}$ to $I_{6}^{S}$ with $B_{d}=0$

\begin{tabular}{lccccccccc}
\hline & & $R^{2}$ & & \multicolumn{3}{c}{ Optimality Gap Entire State Space } \\
Instance & Features 1 & Features 2 & Features 3 & \multicolumn{2}{c}{ Features 1 } & \multicolumn{2}{c}{ Features 2 } & \multicolumn{2}{c}{ Features 3 } \\
& & & & Avg. & MSE & Avg. & MSE & Avg. & MSE \\
\hline $\mathrm{I}_{1}^{S}$ & 0.632 & 0.686 & 0.554 & $5.6 \%$ & 24304 & $5.9 \%$ & 26306 & $5.6 \%$ & 23405 \\
$\mathrm{I}_{1}^{S}$-st & 0.890 & 0.892 & 0.890 & $2.1 \%$ & 7820 & $2.1 \%$ & 7503 & $2.1 \%$ & 7373 \\
\hline $\mathrm{I}_{2}^{S}$ & 0.636 & 0.684 & 0.555 & $6.6 \%$ & 35144 & $7.7 \%$ & 44680 & $6.8 \%$ & 35260 \\
$\mathrm{I}_{2}^{S}$-st & 0.895 & 0.895 & 0.895 & $10.1 \%$ & 147860 & $10.9 \%$ & 172340 & $11.0 \%$ & 175301 \\
\hline $\mathrm{I}_{3}^{S}$ & 0.661 & 0.709 & 0.589 & $3.3 \%$ & 19368 & $3.4 \%$ & 19924 & $3.3 \%$ & 19258 \\
$\mathrm{I}_{3}^{S}$-st & 0.942 & 0.919 & 0.916 & $4.6 \%$ & 58803 & $4.5 \%$ & 58250 & $4.5 \%$ & 62550 \\
\hline $\mathrm{I}_{4}^{S}$ & 0.637 & 0.698 & 0.563 & $5.3 \%$ & 21043 & $5.5 \%$ & 20765 & $5.5 \%$ & 20407 \\
$\mathrm{I}_{4}^{S}$-st & 0.922 & 0.877 & 0.875 & $3.5 \%$ & 19902 & $2.3 \%$ & 5810 & $2.3 \%$ & 5869 \\
\hline $\mathrm{I}_{5}^{S}$ & 0.640 & 0.683 & 0.558 & $2.8 \%$ & 15030 & $2.8 \%$ & 14735 & $3.0 \%$ & 16018 \\
$\mathrm{I}_{5}^{S}$-st & 0.944 & 0.915 & 0.915 & $3.5 \%$ & 38799 & $3.3 \%$ & 34985 & $3.3 \%$ & 34547 \\
\hline $\mathrm{I}_{6}^{S}$ & 0.639 & 0.691 & 0.561 & $9.9 \%$ & 59733 & $11.4 \%$ & 78208 & $10.4 \%$ & 67544 \\
$\mathrm{I}_{6}^{S}$-st & 0.922 & 0.876 & 0.874 & $4.8 \%$ & 30960 & $4.4 \%$ & 28329 & $4.1 \%$ & 26624 \\
\hline
\end{tabular}

more valuable to go to. We elaborate more on the challenges of ADP design, in Section 2.7.

The two aforementioned evaluations (i.e., $R^{2}$ and optimality gap) of the various sets of features consider all states of the MDP. A third approach to evaluate the performance of an ADP design and decide which basis functions to use, which is applicable to large instances, is to pick some initial state (or multiple initial states) and compare (i) the values learned by the ADP algorithm and (ii) the resulting performance of the policy resulting from these values. In Chapter 3, we design a categorization of states to thoroughly and systematically evaluate the performance of our ADP approach. In this chapter, we limit ourselves to an evaluation based on two states for the single-trip problem introduced in the experimental setup, and show the results in Figures 2.2 and 2.3. For all sets of features, the ADP algorithm runs for 250 iterations. After each iteration $n$, we fix the learned policy (i.e., weights of the basis functions) and simulate its use for 100 iterations. We repeat this procedure ten times, and plot the average costs for the VFA at iteration $n$.

In Figures 2.2 and 2.3, we show two additional ADP designs: a look-up table approach and an $\epsilon$-greedy approach. In a look-up table approach, as the name indicates, ADP updates the value (costs) of each state that is observed within the algorithm rather than updating the weights of a parametrized function, such as in the case of the features approach. On the one hand, this approach avoids the challenge on designing features. On the other hand, the increasing number of states makes it impossible to use in large problem instances. We include it in our tests for exemplifying potential benefits and pitfalls of this common look-up approach. In an $\epsilon$-greedy approach, ADP makes random decisions, during the stages and iterations of the learning phase, with a certain probability $(\epsilon=0.05$ in our case). This so-called "exploration" approach can be useful during the learning phase of ADP in problems with many local-optima. We include it in our tests for exemplifying additional mechanisms to improve the learned values of ADP. We elaborate on the $\epsilon$-greedy approach in more 
detail in Chapter 4, where exploration appears to be crucial for a good performance of ADP.
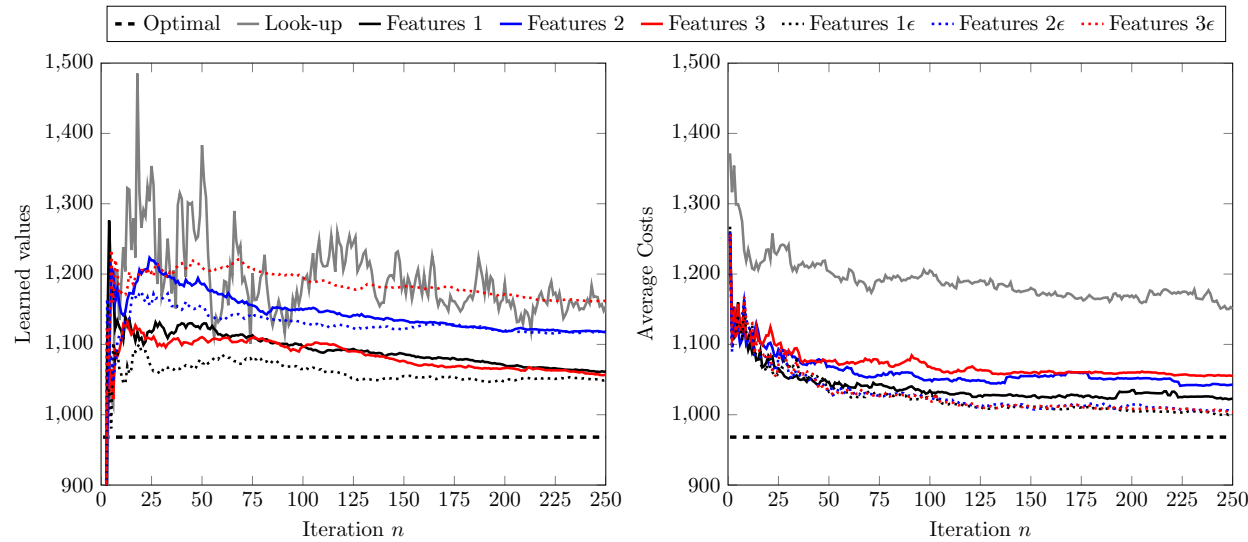

Figure 2.2: Learned values (left) and average cost performance (right) of the ADP algorithm for the different sets of features for State 1 of the single-trip problem.
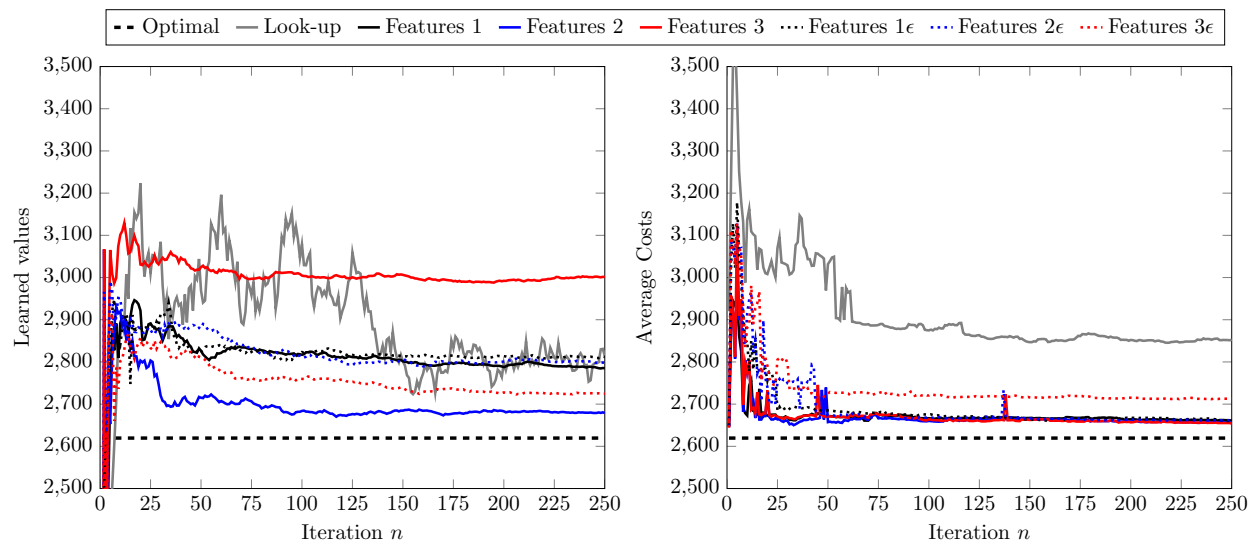

Figure 2.3: Learned values (left) and average cost performance (right) of the ADP algorithm for the different sets of features for State 2 of the single-trip problem.

For State 1 in Figure 2.2, we observe some differences among the learned values and the average costs of the resulting policy of the three Features. These differences can lead to choosing a different winning set of features. On the one hand, the differences among the learned values of the three sets of features indicate that Features $1 \epsilon$ is the best. On the other hand, there are no clear differences among the average costs of the three Features with $\epsilon$, indicating that the three sets perform equally well when using the $\epsilon$-greedy approach 
(and all better than all no- $\epsilon$ Features). Furthermore, we observe that the $\epsilon$-greedy approach improves the learned values in Features 1 and Features 2, but not in Features 3. In the case of the average costs, the $\epsilon$-greedy approach improves all sets of features in a way that their performance is almost the same. In both the learned values and the average costs of the resulting policy, Look-up performs the worse.

The results for State 1 can lead to the conclusion that a proper tuning of the exploration/exploitation tradeoff (e.g., via the $\epsilon$-greedy) can have a larger impact on the performance than the set of features chosen. However, an explanation on why this is the case for this state has to do with the state and the sets of features themselves. State 1 is an almost empty state (i.e., only one freight), which means most basis functions of the three sets we test return zero. Remind that the updating algorithm can only determine how significant the weight of a basis function is as long as it observes it. When only one basis function is observed, and this basis function behaves similarly in all sets of features, the updating algorithm will assign similar weights and thus the resulting policies will be approximately the same.

For State 2 in Figure 2.3, we observe significant differences among the learned values of the Features, but not among the average costs of the resulting policies. Clearly, Features 2 and Features $3 \epsilon$ have the two best learned values, and Features 3 the worst. However, when looking at the average costs, the policy from all three Features (without the $\epsilon$-greedy approach) seem to achieve the same costs, between $1 \%$ and $2 \%$ away from the optimal costs. Moreover, the second-best learning set of features, Features $3 \epsilon$, is now performing secondworst of all sets of features tested. This indicates that having a good learned value of a state does not necessarily result in a good performance. Although Look-up has better learned values in State 2 than in State 1, it still has the worst average costs of all ADP designs tested.

In Figure 2.4, we show the average difference between the average costs of the simulation of the resulting policy and the optimum expected costs of each state, for all states in Instance $\mathrm{I}_{1}^{S}$-st. Overall, we observe that the relative ranking of the three sets of features is good, i.e., states with a low expected cost in the MDP solution also have low simulated costs of the ADP policy learned. We observe that differences in the three sets of features arise mostly in the bottom half of the values, i.e., below the average cost across states. The average difference across all states, between the optimum costs and ADP's average costs is $2.67 \%$ for Features 1, 2.45\% for Features 2, and 2.36\% for Features 3. The Look-up approach is $7.50 \%$ away from optimum on average, making it worse than any of the three sets of features tested.

Overall, there are several ways to evaluate the performance of different sets of features. Naturally, to select the "best" set, several design characteristics must be considered, among which the optimality gap, error spread, and computational time. We select Features 3 as the best one for our ADP approach for two reasons: (i) it only contains linear features, which make it directly applicable to be used in the MILP for the single-stage decision, and (ii) it contains the least number of features, improving the computational time of the updating function within the ADP algorithm. We will use this third set of features for the following chapter. For now, we briefly discuss design and evaluation challenges in ADP with basis functions. 


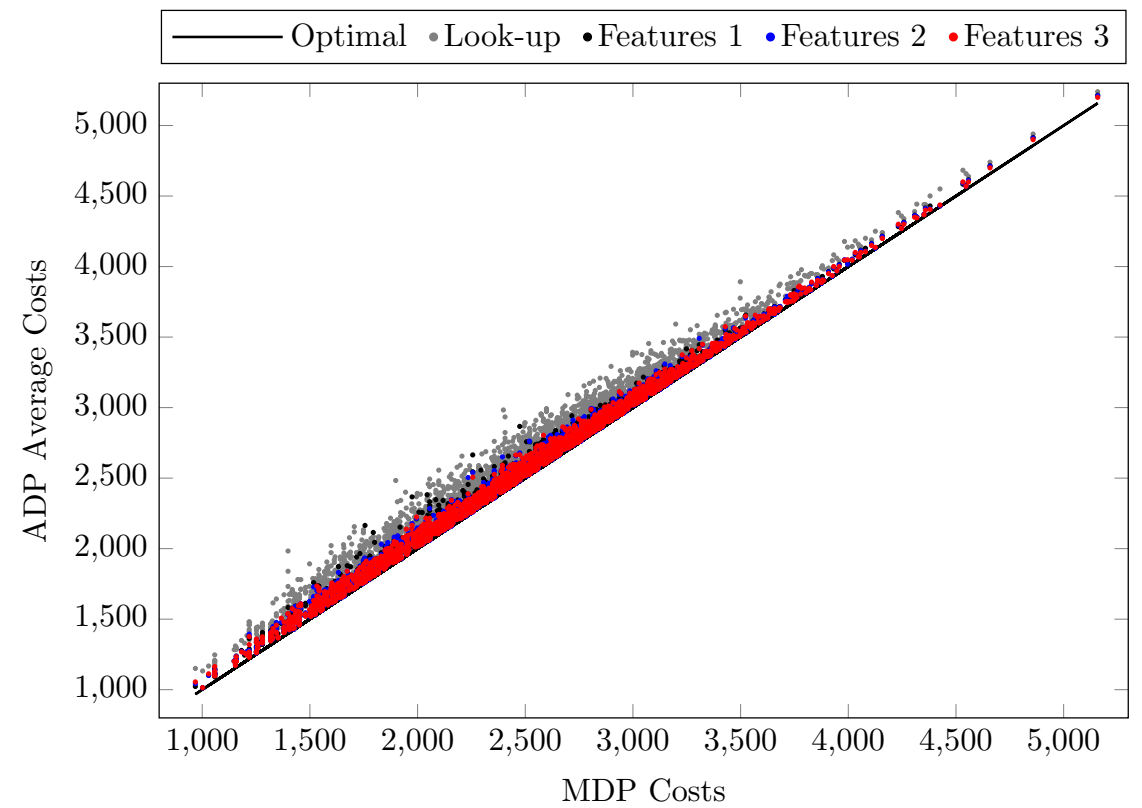

Figure 2.4: Graphical comparison of optimal values against average costs of all states in Instance $I_{1}^{S}$-st.

\subsection{Discussion}

There are several challenges in constructing a close-to-optimal ADP algorithm. When looking at our results, we can conclude that deciding on which set of features to use requires careful design and testing. Next to the challenge of designing "features" of a state, there is a challenge in estimating their accuracy. The coefficient of determination $R^{2}$ is one of many statistical measures one could use to conjecture the performance of a design in a fast way. However, as shown in our experiments, the results of such a statistical measure are not generalizable to judge the performance of a set of features for different network settings. Another option for estimating a design's accuracy, which is computationally more intensive, is to test the designs using simulation of small problem instances that can be solved to optimality. Although this method allows for a deeper analysis of the differences among ADP designs with respect to the optimal solution, it is possible that not all significant interactions among the stochastic variables are present in such instances. Without those relations in the accuracy tests, results can differ in larger instances, which are common in practice.

Furthermore, we observed that the quality of the chosen set of features was heavily problem-state dependent. An explanation on this situation has to do with two characteristics 
of how the basis functions approximate the future costs. First, all weights of the basis functions, which determine the output policy of the ADP algorithm, can only be updated (i.e., improved) as long as the basis functions are non-zero. In our last two-state analysis, we observe that the performance of all sets of features is significantly better in State 2, which contains many basis functions with a non-zero value, than in State 1, which contains mostly basis functions with a value of zero. Second, values of basis functions that fluctuate a lot might harm the approximation in early iterations. The error used to update can be too large when the value of a basis function is large and its corresponding weight is poorly estimated. In State 2, the difference between the best and worst set of features is larger than in State 1. In this example problem, 1.2 freights arrive on average per day (with at most two freights). This means that State 1 is a state one can expect on average whereas State 2 is an exceptionally busy state. Additionally, with the short horizon considered in this problem, the initial conditions (state) can have a large impact on the optimal costs. Thus, problem and state characteristics must be considered when using the basis functions approach.

\subsection{Conclusions}

In this chapter, we developed an MDP model for the anticipatory scheduling of freight in long-haul round-trips within a synchromodal transport network, and designed an ADP algorithm to solve it. We analyzed the optimality gap of our ADP approach, under various problem settings, and provided methodological insights on developing a close-to-optimal ADP algorithm. Two observations stand out. First, we showed that a careful analysis is required to evaluate the accuracy of different $\mathrm{ADP}$ designs to approximate the optimal solution of the MDP model they are designed for. Specifically, we showed that additional mechanisms besides statistical inference and simulation are needed. Second, we showed that ADP performs better in some states and problem instances than others. These differences in performance show that problem characteristics must be considered when designing a solution algorithm. In the following chapter, we continue the analysis of our ADP approach using larger problem instances with various uncertainty profiles.

\section{Appendices}

\section{2-A Number of Realizations of the Exogenous Information}

Each realization $\omega \in \Omega$ is basically a combination of the values that the exogenous information variables $\widetilde{F}_{t, d, r, k}$ and $\widetilde{G}_{t, d, r, k}$ can have. Since both $\widetilde{F}_{t, d, r, k}$ and $\widetilde{G}_{t, d, r, k}$ can have a value greater than one due to multiple freights having the same characteristics, the number of possible realizations of exogenous information $|\Omega|$ depends not only on the number of different characteristics, but also on the number of freights that can have the same 
characteristics, as seen in (2.17).

$$
\begin{aligned}
|\Omega| & =\sum_{n=0}^{|\mathcal{F}|}\left(\begin{array}{c}
|\mathcal{D}| \cdot|\mathcal{R}| \cdot|\mathcal{K}|+n-1 \\
n
\end{array}\right) \cdot \sum_{n=0}^{|\mathcal{G}|}\left(\begin{array}{c}
|\mathcal{D}| \cdot|\mathcal{R}| \cdot|\mathcal{K}|+n-1 \\
n
\end{array}\right) \\
& =\sum_{n=0}^{|\mathcal{F}|} \frac{(|\mathcal{D}| \cdot|\mathcal{R}| \cdot|\mathcal{K}|+n-1) !}{n !(|\mathcal{D}| \cdot|\mathcal{R}| \cdot|\mathcal{K}|-1) !} \cdot \sum_{n=0}^{|\mathcal{G}|} \frac{(|\mathcal{D}| \cdot|\mathcal{R}| \cdot|\mathcal{K}|+n-1) !}{n !(|\mathcal{D}| \cdot|\mathcal{R}| \cdot|\mathcal{K}|-1) !}
\end{aligned}
$$

In a similar way to $\Omega$, the state space $\mathcal{S}$ grows faster than exponential with an increasing number of possible freight characteristics. The set of possible decisions $\mathcal{X}$ grows fast as well, but the optimal action can be obtained (for realistic problems and in reasonable time) through an Integer Linear Program (ILP), as we show later on. Due to these dimensionality issues, an exact solution is only feasible in small problem instances.

\section{2-B Single-stage Decision Problem}

The single-stage decision problem can be formulated as a MILP, as seen in (2.18a-2.18w).

$$
\begin{gathered}
\min C\left(S_{t}^{n}, x_{t}^{n}\right)=\sum_{\mathcal{D}^{\prime} \subseteq \mathcal{D}}\left(C_{\mathcal{D}^{\prime}} \cdot w_{t, \mathcal{D}^{\prime}}\right)+\sum_{d \in \mathcal{D}}\left(A_{d} \cdot z_{t, d}\right) \\
+\sum_{d \in \mathcal{D}} \sum_{k \in \mathcal{K}}\left(B_{d} \cdot\left(x_{t, d, k}^{F}+x_{t, d, k}^{G}\right)\right)+\sum_{a \in \mathcal{A}}\left(\theta_{a} \cdot \phi_{a}\left(S_{t}^{n, x}\right)\right) \\
\text { s.t. } \\
\sum_{d \in \mathcal{D} k \in \mathcal{K}} \sum_{t, d, k} \leq Q \\
\sum_{d \in \mathcal{D} k \in \mathcal{K}} \sum_{t, d, k} \leq Q \\
\sum_{t, d, 0}^{F}+x_{t, d, 0}^{G}+z_{t, d}=F_{t, d, 0,0}^{n}+G_{t, d, 0,0}^{n}, \forall d \in \mathcal{D} \\
\sum_{k \in \mathcal{K}} x_{t, d, k}^{F}-\sum_{k \in \mathcal{K}}\left(F_{t, d, 0, k}\right) \cdot y_{d} \leq 0, \forall d \in \mathcal{D} \\
\sum_{k \in \mathcal{K}} x_{t, d, k}^{G}-\sum_{k \in \mathcal{K}}\left(G_{t, d, 0, k}\right) \cdot y_{d} \leq 0, \forall d \in \mathcal{D} \\
x_{t, d, k+1}^{F}+F_{t+1, d, 0, k}^{n, x}=F_{t, d, 0, k+1}^{n}+F_{t, d, 1, k}^{n}, \forall d \in \mathcal{D}, k \in \mathcal{K} \mid k<K^{\max } \\
x_{t, d, k+1}^{G}+G_{t+1, d, 0, k}^{n, x}=G_{t, d, 0, k+1}^{n}+G_{t, d, 1, k}^{n}, \forall d \in \mathcal{D}, k \in \mathcal{K} \mid k<K^{\max } \\
F_{t+1, d, 0, K^{\max }}^{n, x}=F_{t, d, 1, K^{\max }}^{n} \forall d \in \mathcal{D} \\
G_{t+1, d, 0, K^{\max }}^{n, x}=G_{t, d, 1, K^{\max } \forall d \in \mathcal{D}}^{n} \forall \\
F_{t+1, d, r, k}^{n, x}=F_{t, d, r+1, k}^{n} \forall d \in \mathcal{D}, k \in \mathcal{K}, r \in \mathcal{R} \mid r<R^{\text {max }} \\
G_{t+1, d, r, k}^{n, x}=G_{t, d, r+1, k}^{n} \forall d \in \mathcal{D}, k \in \mathcal{K}, r \in \mathcal{R} \mid r<R^{\text {max }}
\end{gathered}
$$




$$
\begin{gathered}
w_{t, \mathcal{D}^{\prime}}-y_{t, d^{\prime}} \leq 0, \forall \mathcal{D}^{\prime} \subseteq \mathcal{D}, d^{\prime} \in \mathcal{D}^{\prime} \\
w_{t, \mathcal{D}^{\prime}}+y_{t, d^{\prime}} \leq 1, \forall \mathcal{D}^{\prime} \subseteq \mathcal{D}, d^{\prime} \in \mathcal{D} \backslash \mathcal{D}^{\prime} \\
w_{t, \mathcal{D}^{\prime}}-\sum_{d^{\prime} \in \mathcal{D}^{\prime}} y_{t, d^{\prime}}+\sum_{d^{\prime \prime} \in \mathcal{D}^{\prime} \backslash \mathcal{D}} y_{t, d^{\prime \prime}} \geq 1-\left|\mathcal{D}^{\prime}\right|, \forall \mathcal{D}^{\prime} \subseteq \mathcal{D} \\
\sum_{\mathcal{D}^{\prime} \subseteq \mathcal{D}} w_{t, \mathcal{D}^{\prime}}=1 \\
x_{t, d, k}^{F} \in \mathbb{Z} \cap\left[0, F_{t, d, 0, k}^{n}\right], \forall d \in \mathcal{D}, k \in \mathcal{K} \\
x_{t, d, k}^{G} \in \mathbb{Z} \cap\left[0, F_{t, d, 0, k}^{n}\right], \forall d \in \mathcal{D}, k \in \mathcal{K} \\
y_{t, d} \in\{0,1\}, \forall d \in \mathcal{D} \\
z_{t, d} \in\left[0, F_{t, d, 0,0}^{n}+G_{t, d, 0,0}^{n}\right], \forall d \in \mathcal{D} \\
w_{t, \mathcal{D}^{\prime}} \in[0,1], \forall \mathcal{D}^{\prime} \subseteq \mathcal{D} \\
F_{t+1, d, 0, k}^{n, x} \in\left[0, F_{t, d, 0, k+1}^{n}+F_{t, d, 1, k}^{n}\right], \forall d \in \mathcal{D}, k \in \mathcal{K} \mid k<K^{\max } \\
G_{t+1, d, 0, k}^{n, x} \in\left[0, G_{t, d, 0, k+1}^{n}+G_{t, d, 1, k}^{n}\right], \forall d \in \mathcal{D}, k \in \mathcal{K} \mid k<K^{\max }
\end{gathered}
$$

The objective in (2.18a) is to minimize the sum of (i) a linearized version of the currentday costs of a decision, as shown in (2.3), and (ii) the approximated next-stage cost. Constraints $(2.18 \mathrm{~b})$ to $(2.18 \mathrm{f})$ define the feasible decision space and the auxiliary variables. Constraints $(2.18 \mathrm{~g})$ to $(2.18 \mathrm{l})$ define the post-decision freight variables. Constraints $(2.18 \mathrm{~m})$ to $(2.18 \mathrm{p})$ linearize costs through the use of a binary variable $w_{t, \mathcal{D}^{\prime}}$ that gets a value of 1 if the subset of destinations $\mathcal{D}^{\prime}$ is visited with the long-haul vehicle and 0 otherwise. Constraints $(2.18 \mathrm{q})$ to $(2.18 \mathrm{w})$ define the domain of all variables in the MILP. Note that not all post-decision freight variables are included in the model, only the ones that are affected by the decision. Furthermore, the basis functions $\phi_{a}\left(S_{t}^{n, x}\right)$ for all $a \in \mathcal{A}$ are assumed to be linear in the decision variables of the MILP model. For examples on how to incorporate basis functions in the MILP, see 2-C.

\section{2-C Basis Functions in the MILP}

To model the various sets of features seen in Table 2.2 of the main text, we introduce two additional variables into the MILP shown in $(2.18 \mathrm{a})$ through $(2.18 \mathrm{w})$. The binary variable $u_{t, d}$ gets a value of 1 if destination $d$ has any MustGo freight, and 0 otherwise. The binary variable $v_{t, d}$ gets a value of 1 if destination $d$ has any MayGo freight, and 0 otherwise. To define these variables within the MILP, we introduce constraints $(2.19 \mathrm{~b})$ through $(2.19 \mathrm{~g})$. To account for the future costs (i.e., weights from the ADP algorithm), we need to construct weights for the MILP decision variables depending on the set of features $\mathcal{A}$ used, as shown in (2.19a). For example, in VFA 2, we have the feature "Indicator of MustGo freights per destination", whereas in VFA 3 we do not have this feature. In both VFA 2 and VFA 3 we have the feature "Count of MustGo destinations". When using VFA 2, the weight $\theta_{d}^{u, \mathcal{A}}$ in the MILP will be the sum of weights corresponding to the feature $a$ "Indicator of MustGo freights per destination" 
and the feature $a^{\prime}$ "Count of MustGo destinations" (i.e., $\theta_{d}^{u, \mathcal{A}}=\theta_{a}+\theta_{a^{\prime}}$ ). When using VFA 3 , the weight $\theta_{d}^{u, \mathcal{A}}=\theta_{a^{\prime}}$, since this set of features only has the feature $a^{\prime}$ "Count of MustGo destinations", related to the variable $u_{t, d}$. At the end of $(2.19 \mathrm{a})$, we add the constant $\delta_{t}^{n}$, which takes into account the total costs of features that are not dependent on the MILP variables, but only on the state itself (i.e., "Number of future freights", "Constant", etc.).

$$
\begin{aligned}
& \sum_{a \in \mathcal{A}}\left(\theta_{a} \cdot \phi_{a}\left(S_{t}^{n, x}\right)\right)=\left[\theta_{d, k}^{F, \mathcal{A}} \cdot F_{t+1, d, 0, k}^{n, x}\right]_{\forall d \in \mathcal{D}, k \in \mathcal{K}}+\left[\theta_{d, k}^{G, \mathcal{A}} \cdot G_{t+1, d, 0, k}^{n, x}\right]_{\forall d \in \mathcal{D}, k \in \mathcal{K}} \\
& +\left[\theta_{d}^{u, \mathcal{A}} \cdot u_{t, d}\right]_{\forall d \in \mathcal{D}}+\left[\theta_{d}^{v, \mathcal{A}} \cdot v_{t, d}\right]_{\forall d \in \mathcal{D}} \\
& +\delta_{t}^{n} \\
& F_{t+1, d, 0,0}^{n, x}-\left(F_{t, d, 0,1}^{n}+F_{t, d, 1,0}^{n}\right) \cdot u_{t, d} \leq 0, \forall d \in \mathcal{D} \\
& G_{t+1, d, 0,0}^{n, x}-\left(G_{t, d, 0,1}^{n}+G_{t, d, 1,0}^{n}\right) \cdot u_{t, d} \leq 0, \forall d \in \mathcal{D} \\
& \sum_{k \in \mathcal{K} \mid k>0} F_{t+1, d, 0, k}^{n, x}-\left(\sum_{k \in \mathcal{K} \backslash K^{\max }}\left(F_{t, d, 0, k+1}^{n}\right)+\sum_{k \in \mathcal{K}}\left(F_{t, d, 1, k+1}^{n}\right)\right) \cdot v_{t, d} \leq 0 \\
& \forall d \in \mathcal{D} \\
& \sum_{k \in \mathcal{K} \mid k>0} G_{t+1, d, 0, k}^{n, x}-\left(\sum_{k \in \mathcal{K} \backslash K^{\max }}\left(G_{t, d, 0, k+1}^{n}\right)+\sum_{k \in \mathcal{K}}\left(G_{t, d, 1, k+1}^{n}\right)\right) \cdot v_{t, d} \leq 0 \\
& \forall d \in \mathcal{D} \\
& u_{t, d} \in\{0,1\}, \forall d \in \mathcal{D} \\
& v_{t, d} \in\{0,1\}, \forall d \in \mathcal{D}
\end{aligned}
$$



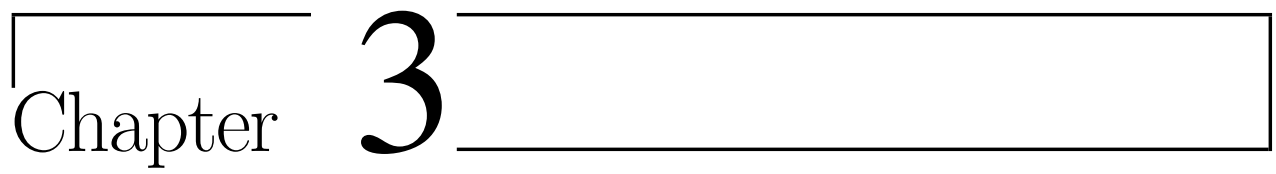

\section{Long-haul Round-trip Transport: Solution Analysis}

In this chapter, we analyze the practical relevance of our approach to solve the long-haul round-trip scheduling problem presented in the previous chapter. In addition, we also illustrate the practical relevance of our approach in the simpler single-trip problem described and exemplified in the previous chapters, and describe its relation to the round-trip problem. Furthermore, we provide managerial insights for several round-trip settings about the potential cost reductions of our approach compared to single-period or myopic optimization. This chapter is based on parts of our publications in $[69,71]$ and assumes the reader is familiar with Chapter 2, of this thesis.

\subsection{Introduction}

As introduced earlier, the scheduling problem we study in this chapter is motivated by a Dutch LSP that transports containers between the Eastern part of the country to the Port of Rotterdam in daily round-trips using a barge. Similar scheduling problems can be found in the so-called point-to-point [54], line [54], and corridor [13] network topologies of intermodal transport under the synchromodal characteristics. Naturally, each network topology and problem instance has different settings that influence the scheduling and the attainable performance. Some have tight capacities compared to the demand, some others have more uncertainty on the destinations that the high-capacity mode has to visit given the freight demand. In this chapter, we analyze the performance of our ADP approach for larger instances compared to those of Chapter 2, under different problem settings. These larger instances cannot longer be solved to optimality in reasonable time, and hence we compare to a benchmark heuristic.

Our goal in this chapter is to provide insight into the effect of various problem charac- 
teristics on the anticipatory freight selection decisions using the ADP approach defined in Chapter 2. For this reason, this chapter does not include a section with a mathematical model or solution algorithm to the problem. It begins with the experimental setup in Section 3.2 and continues with the numerical results in Section 3.3. Section 3.4 contains an interlude with experiments for a simplified version of the problem: single-trip transport. The simplified version of the problem, which was explained in the previous chapter, is used again in Chapter 7. In Section 3.5, the strengths and weaknesses of the ADP approach over the several uncertainty profiles are discussed. Finally, we conclude in Section 3.6 with the main insights of the analysis in this chapter and directions for further research.

\subsection{Experimental Setup}

In the following analysis, we compare our ADP approach with Features 3 described in Table 2.2 of Chapter 2 against a benchmark heuristic. The benchmark heuristic consists of the policy to optimize the single-period costs while maximizing the use of the high-capacity mode capacity. It consists of two steps. First, all urgent freights are assigned to the high-capacity mode or the alternative mode such that minimum costs are achieved that day. Once the urgent freights are assigned, the heuristic tries to fill in the high-capacity vehicle with freights that do not increase the costs (e.g., freights to the same destinations of the selected ones). In a similar way to the experimental setup in Chapter 2 (Section 2.5), we assess the differences between in different instances representing different networks. The parameters in these instances have similar values and probability distributions as the Dutch LSP characteristics. For sensitivity analysis, we modify the probability distributions per instance to represent different LSPs or modes traveling to different parts of the network by a single LSP. The probability distributions for these large instances, named $\mathrm{I}_{1}^{L}$ to $\mathrm{I}_{6}^{L}$, are shown in Table 3.1. The time horizon is $T^{\max }=5$ and the vehicle capacity is $Q=10$ for all six instances. Although 10 freights seem small compared to the average capacity of a barge of 48 containers, we note that freights can represent orders with multiple containers for a barge (in the case of the Dutch LSP, freight orders usually have at least 4 containers which must be transported together). The ranges for the costs are the same for all six instances, and are defined as follows: $C_{\mathcal{D}^{\prime}}=[250,2150], B_{d}=[50,150]$, and $A_{d}=[300,800]$. The cost setup is based upon the same three considerations as in Section 2.5.

Instances $I_{1}^{L}$ to $I_{6}^{L}$ differ with respect to the input probability distributions. The rationale behind this difference is to test three characteristics of a network: (i) the "balance" of delivery and pickup freight destinations, (ii) the number of pre-announced orders, i.e., freights whose release-day is in the future, and (iii) the "urgency" of freights, whether they must be carried the same day they are announced or they can be carried some days later. In this rationale, $\mathrm{I}_{1}^{L}$ represents a totally balanced network, while $\mathrm{I}_{2}^{L}$ represents a totally unbalanced network. Instances $I_{3}^{L}$ through $\mathrm{I}_{6}^{L}$ represent balanced networks with different time-window characteristics. Freights are mostly released immediately in $\mathrm{I}_{3}^{L}$, whereas in $\mathrm{I}_{4}^{L}$ they are mostly released in the future. $\mathrm{In} \mathrm{I}_{5}^{L}$, freights are mostly urgent, i.e., immediate release and due-day, whereas in $\mathrm{I}_{6}^{L}$ freights have future release and due-day.

We use a two-step methodology to measure differences in performance between the policy 
Table 3.1: Input parameters of the numerical experiments

\begin{tabular}{|c|c|c|c|c|c|c|}
\hline \multirow{2}{*}{ Parameter } & \multicolumn{6}{|c|}{ Instance } \\
\hline & $\mathrm{I}_{1}^{L}$ & $\mathrm{I}_{2}^{L}$ & $\mathrm{I}_{3}^{L}$ & $\mathrm{I}_{4}^{L}$ & $\mathrm{I}_{5}^{L}$ & $\mathrm{I}_{6}^{L}$ \\
\hline $\mathcal{D}$ & $\{1,2, \ldots, 12\}$ & $\{1,2, \ldots, 12\}$ & $\{1,2, \ldots, 12\}$ & $\{1,2, \ldots, 12\}$ & $\{1,2, \ldots, 12\}$ & $\{1,2, \ldots, 12\}$ \\
\hline $\begin{array}{l}p_{d}^{\mathrm{D}, \mathrm{F}} \\
p_{d}^{\mathrm{D}, \mathrm{G}}\end{array}$ & $\begin{array}{l}\approx \frac{6^{d}}{d !} e^{-6} \\
\approx \frac{6^{d}}{d !} e^{-6}\end{array}$ & $\begin{array}{c}\approx \frac{6^{d}}{d !} e^{-6} \\
\frac{1}{12}, \forall d \in \mathcal{D}\end{array}$ & $\begin{array}{l}\approx \frac{6^{d}}{d !} e^{-6} \\
\approx \frac{6^{d}}{d !} e^{-6}\end{array}$ & $\begin{array}{l}\approx \frac{6^{d}}{d !} e^{-6} \\
\approx \frac{6^{d}}{d !} e^{-6}\end{array}$ & $\begin{array}{l}\approx \frac{6^{d}}{d !} e^{-6} \\
\approx \frac{6^{d}}{d !} e^{-6}\end{array}$ & $\begin{array}{l}\approx \frac{6^{d}}{d !} e^{-6} \\
\approx \frac{6^{d}}{d !} e^{-6}\end{array}$ \\
\hline $\mathcal{R}$ & $\{0,1,2\}$ & $\{0,1,2\}$ & $\{0,1,2\}$ & $\{0,1,2\}$ & $\{0,1,2\}$ & $\{0,1,2\}$ \\
\hline $\begin{array}{l}p_{r}^{\mathrm{R}, \mathrm{F}} \\
p_{r}^{\mathrm{R}, \mathrm{G}}\end{array}$ & $\left\{\begin{array}{l}\left.\frac{3}{10}, \frac{3}{10}, \frac{4}{10}\right\} \\
\left.\frac{3}{10}, \frac{3}{10}, \frac{4}{10}\right\}\end{array}\right\}$ & $\left\{\begin{array}{l}\frac{3}{10}, \frac{3}{10}, \frac{4}{10} \\
\left.\frac{3}{10}, \frac{3}{10}, \frac{4}{10}\right\}\end{array}\right\}$ & $\left\{\begin{array}{l}\frac{8}{10}, \frac{1}{10}, \frac{1}{10} \\
\frac{8}{10}, \frac{1}{10}, \frac{1}{10}\end{array}\right\}$ & $\left\{\begin{array}{l}\frac{1}{10}, \frac{1}{10}, \frac{8}{10} \\
\frac{1}{10}, \frac{1}{10}, \frac{8}{10}\end{array}\right\}$ & $\left\{\begin{array}{l}\frac{8}{10}, \frac{1}{10}, \frac{1}{10} \\
\frac{8}{10}, \frac{1}{10}, \frac{1}{10}\end{array}\right\}$ & $\left\{\begin{array}{l}\frac{1}{10}, \frac{1}{10}, \frac{8}{10} \\
\frac{1}{10}, \frac{1}{10}, \frac{8}{10}\end{array}\right\}$ \\
\hline $\mathcal{K}$ & $\{0,1,2\}$ & $\{0,1,2\}$ & $\{0,1,2\}$ & $\{0,1,2\}$ & $\{0,1,2\}$ & $\{0,1,2\}$ \\
\hline $\begin{array}{l}p_{k}^{\mathrm{K}, \mathrm{F}} \\
p_{k}^{\mathrm{K}, \mathrm{G}}\end{array}$ & $\begin{array}{l}\left\{\frac{2}{10}, \frac{3}{10}, \frac{5}{10}\right\} \\
\left\{\frac{2}{10}, \frac{3}{10}, \frac{5}{10}\right\}\end{array}$ & $\begin{array}{l}\left\{\frac{2}{10}, \frac{3}{10}, \frac{5}{10}\right\} \\
\left\{\frac{2}{10}, \frac{3}{10}, \frac{5}{10}\right\}\end{array}$ & $\begin{array}{l}\left\{\frac{2}{10}, \frac{3}{10}, \frac{5}{10}\right\} \\
\left\{\frac{2}{10}, \frac{3}{10}, \frac{5}{10}\right\}\end{array}$ & $\begin{array}{l}\left\{\frac{2}{10}, \frac{3}{10}, \frac{5}{10}\right\} \\
\left\{\frac{2}{10}, \frac{3}{10}, \frac{5}{10}\right\}\end{array}$ & $\begin{array}{l}\left\{\frac{8}{10}, \frac{1}{10}, \frac{1}{10}\right\} \\
\left\{\frac{8}{10}, \frac{1}{10}, \frac{1}{10}\right\}\end{array}$ & $\begin{array}{l}\left\{\frac{1}{10}, \frac{1}{10}, \frac{8}{10}\right\} \\
\left\{\frac{1}{10}, \frac{1}{10}, \frac{8}{10}\right\}\end{array}$ \\
\hline $\mathcal{F}=\mathcal{G}$ & $\{1,2, \ldots, 10\}$ & $\{1,2, \ldots, 10\}$ & $\{1,2, \ldots, 10\}$ & $\{1,2, \ldots, 10\}$ & $\{1,2, \ldots, 10\}$ & $\{1,2, \ldots, 10\}$ \\
\hline$p_{f}^{\mathrm{F}}$ & $\approx \frac{4^{f}}{f !} e^{-4}$ & $\approx \frac{4^{f}}{f !} e^{-4}$ & $\approx \frac{4^{f}}{f !} e^{-4}$ & $\approx \frac{4^{f}}{f !} e^{-4}$ & $\approx \frac{4^{f}}{f !} e^{-4}$ & $\approx \frac{4^{f}}{f !} e^{-4}$ \\
\hline$p_{f}^{G}$ & $\approx \frac{4^{\dot{f}}}{f !} e^{-4}$ & $\approx \frac{2^{\dot{f}}}{f !} e^{-2}$ & $\approx \frac{4^{\dot{f}}}{f !} e^{-4}$ & $\approx \frac{4^{\dot{f}}}{f !} e^{-4}$ & $\approx \frac{4^{\dot{f}}}{f !} e^{-4}$ & $\approx \frac{4^{\dot{f}}}{f !} e^{-4}$ \\
\hline
\end{tabular}

resulting from the ADP algorithm and the benchmark heuristic. However, instead of doing the steps for all states as we did in the previous chapter, we do it for a subset of states. This subset is chosen based on a categorization of states according that follows two criteria: (i) number of released freights and (ii) number of destinations. Each criteria is divided into three levels, for a total of 9 categories, as we explain in the next section. The two steps to measure performance are then applied to one state per category, for each instance. The state is chosen randomly among those states close to the center of the cluster of states from the given category, in terms of the two aforementioned criteria for each category. In the first step, we apply the ADP algorithm to each of these states, using the Features 3 and the settings found in Section 2.5. In the second step, we simulate the use of the policy resulting from the ADP algorithm and compare this with the benchmark heuristic. We use common random numbers for the two simulations in order to allow a pairwise analysis of differences and rule out variability in the arrival of freight as the cause for differences in performance between the ADP policy and the benchmark heuristic.

\subsubsection{Categorization of States in Normal-sized Experiments}

Each instance of in Table 3.1 has much more than $7 \cdot 10^{27}$ states. For this reason, we cannot evaluate the performance for all initial states. However, choosing a subset of the 
entire state space has two complications: (i) the experimental results hold for the chosen sample of states but not necessarily to the entire state space and (ii) states have different characteristics that influence the performance of the ADP algorithm, as we saw in the previous chapter. To measure different regions of the state space in a representative way, we build a subset of states as follows. First, we generate a random sample of 10,000 states for each network $I_{1}^{L}$ to $I_{6}^{L}$, which are "commonly encountered" in such a network. To achieve a commonly encountered state, we simulate the network using the benchmark heuristic. We begin with a random initial state, and each day within the horizon of a week, we simulate the decisions and arrival of new freights. After the simulated week, we stop the simulation and store the state in which the system is at that moment. Naturally, the resulting sample contains various types of states representing various regions of the entire state space. To categorize these regions, we use two state-characteristics that are of great importance in practice: the number of released freights, and the number of different destinations of the released freights. We define three levels in each characteristic: Low, Medium, and High, and categorize the 10,000 states of each instance into nine different categories $\mathrm{C} 1-\mathrm{C} 9$, as seen in Figure 3.1. Since the distribution of state characteristics differ per instance, we define the boundary of the levels individually for each instance as seen in Table 3.2. These boundaries are computed with the objective of minimizing the difference between the category with the largest number of states and the one with the lowest number of states.

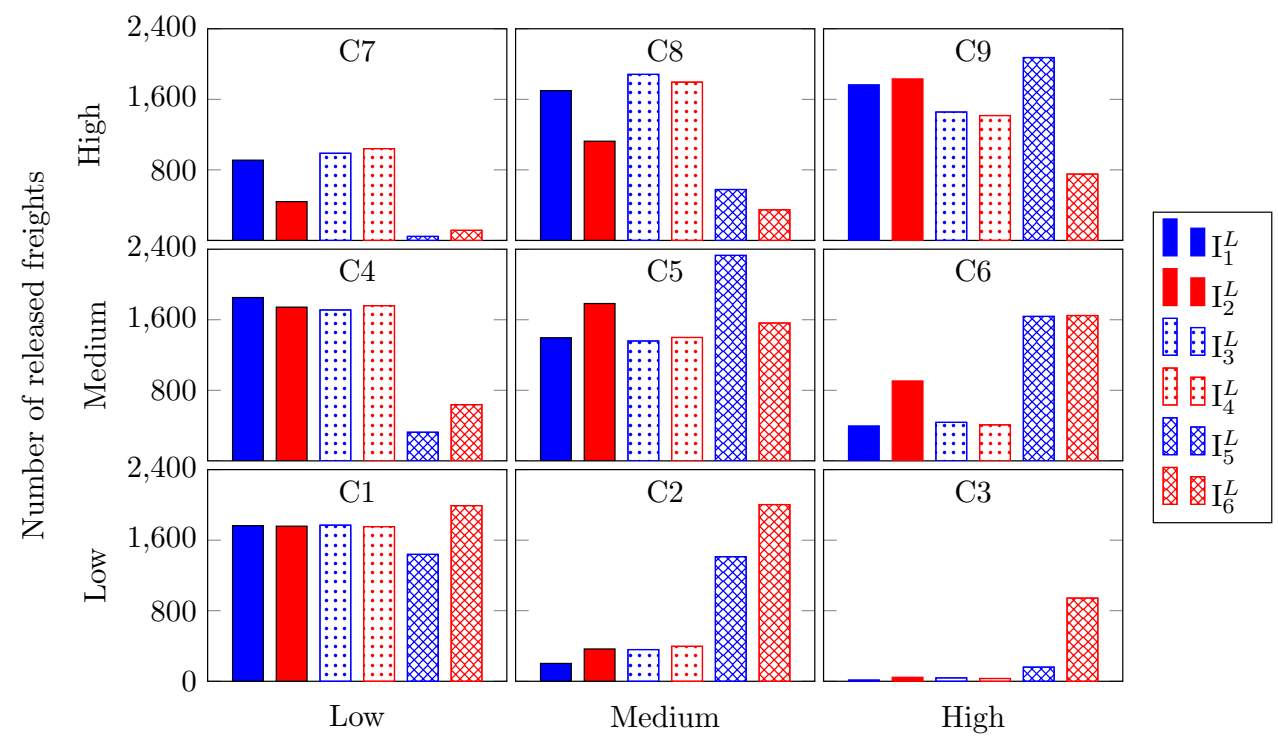

Number of destinations

Figure 3.1: Categorization of the 10,000 sampled states of the Instances $I_{1}^{L}$ to $I_{6}^{L}$ 
Table 3.2: Categorization of states

\begin{tabular}{|c|c|c|c|c|c|c|c|c|c|c|c|c|c|c|}
\hline \multirow{2}{*}{ Category } & \multirow{2}{*}{$\begin{array}{l}\text { Freights } \\
(\mathrm{F})\end{array}$} & \multirow{2}{*}{$\begin{array}{l}\text { Destinations } \\
\text { (D) }\end{array}$} & \multicolumn{2}{|c|}{$\mathrm{I}_{1}^{L}$} & \multicolumn{2}{|c|}{$\mathrm{I}_{2}^{L}$} & \multicolumn{2}{|c|}{$\mathrm{I}_{3}^{L}$} & \multicolumn{2}{|c|}{$\mathrm{I}_{4}^{L}$} & \multicolumn{2}{|c|}{$\mathrm{I}_{5}^{L}$} & \multicolumn{2}{|c|}{$\mathrm{I}_{6}^{L}$} \\
\hline & & & $\mathrm{F}$ & D & $\mathrm{F}$ & D & $\mathrm{F}$ & D & $\mathrm{F}$ & D & $\mathrm{F}$ & D & $\mathrm{F}$ & D \\
\hline $\mathrm{C} 1$ & Low & Low & {$[0,13)$} & {$[0,4)$} & {$[0,11)$} & {$[0,4)$} & {$[0,14)$} & {$[0,4)$} & {$[0,14)$} & {$[0,4)$} & {$[0,8)$} & {$[0,2)$} & {$[0,22)$} & {$[0,4)$} \\
\hline $\mathrm{C} 2$ & Low & Medium & {$[0,13)$} & {$[4,5)$} & {$[0,11)$} & {$[4,5)$} & {$[0,14)$} & {$[4,5)$} & {$[0,14)$} & {$[4,5)$} & {$[0,8)$} & {$[2,3)$} & {$[0,22)$} & {$[4,5)$} \\
\hline C3 & Low & High & {$[0,13)$} & {$[5,9)$} & {$[0,11)$} & {$[5,10)$} & {$[0,14)$} & {$[5,8)$} & {$[0,14)$} & {$[5,9)$} & {$[0,8)$} & {$[3,7)$} & {$[0,22)$} & {$[5,9)$} \\
\hline $\mathrm{C} 4$ & Medium & Low & {$[13,20)$} & {$[0,4)$} & {$[11,18)$} & {$[0,4)$} & {$[14,20)$} & {$[0,4)$} & {$[14,20)$} & {$[0,4)$} & {$[8,14)$} & {$[0,2)$} & {$[22,30)$} & {$[0,4)$} \\
\hline C5 & Medium & Medium & {$[13,20)$} & {$[4,5)$} & {$[11,18)$} & {$[4,5)$} & {$[14,20)$} & {$[4,5)$} & {$[14,20)$} & {$[4,5)$} & {$[8,14)$} & {$[2,3)$} & {$[22,30)$} & {$[4,5)$} \\
\hline C6 & Medium & High & {$[13,20)$} & {$[5,9)$} & {$[11,18)$} & {$[5,10)$} & {$[14,20)$} & {$[5,8)$} & {$[14,20)$} & {$[5,9)$} & {$[8,14)$} & {$[3,7)$} & {$[22,30)$} & {$[5,9)$} \\
\hline $\mathrm{C} 7$ & High & Low & {$[20,57)$} & {$[0,4)$} & {$[18,45)$} & {$[0,4)$} & {$[20,52)$} & {$[0,4)$} & {$[20,47)$} & {$[0,4)$} & {$[14,37)$} & {$[0,2)$} & {$[30,54)$} & {$[0,4)$} \\
\hline $\mathrm{C} 8$ & High & Medium & {$[20,57)$} & {$[4,5)$} & {$[18,45)$} & {$[4,5)$} & {$[20,52)$} & {$[4,5)$} & {$[20,47)$} & {$[4,5)$} & {$[14,37)$} & {$[2,3)$} & {$[30,54)$} & {$[4,5)$} \\
\hline C9 & High & High & {$[20,57)$} & {$[5,9)$} & {$[18,45)$} & {$[5,10)$} & {$[20,52)$} & {$[5,8)$} & {$[20,47)$} & {$[5,9)$} & {$[14,37)$} & {$[3,7)$} & {$[30,54)$} & {$[5,9)$} \\
\hline
\end{tabular}

\subsection{Numerical Results}

In this section, we show the performance of the ADP algorithm on the large instances $\mathrm{I}_{1}^{L}$ through $\mathrm{I}_{6}^{L}$. We measure performance in these experiments as the pairwise difference between the simulated costs of using the policy resulting from the ADP algorithm and the simulated costs of using the benchmark heuristic mentioned in Section 3.2. By pairwise difference we mean the use of common random numbers in the replications of the simulations, to rule out variability in the network as a cause for the differences. The differences, as a percentage, for each category of each instance, are shown in Table 3.3. A negative percentage in this table can be interpreted as how much lower costs the ADP approach achieves compared to the heuristic.

Table 3.3: Average cost difference between the ADP policy and the benchmark policy

\begin{tabular}{lccccccc}
\hline Category & $\mathrm{I}_{1}^{L}$ & $\mathrm{I}_{2}^{L}$ & $\mathrm{I}_{3}^{L}$ & $\mathrm{I}_{4}^{L}$ & $\mathrm{I}_{5}^{L}$ & $\mathrm{I}_{6}^{L}$ & Average \\
\hline C1 & $-5.9 \%$ & $-8.6 \%$ & $-9.4 \%$ & $-5.5 \%$ & $-0.6 \%$ & $-5.2 \%$ & $-5.9 \%$ \\
C2 & $-9.1 \%$ & $-12.3 \%$ & $-4.0 \%$ & $-2.7 \%$ & $-0.6 \%$ & $-11.0 \%$ & $-6.6 \%$ \\
C3 & $-1.9 \%$ & $-6.7 \%$ & $-8.2 \%$ & $-3.1 \%$ & $1.1 \%$ & $-7.2 \%$ & $-4.3 \%$ \\
C4 & $-14.9 \%$ & $-25.5 \%$ & $-5.2 \%$ & $-11.8 \%$ & $-1.5 \%$ & $-8.0 \%$ & $-11.2 \%$ \\
C5 & $-15.1 \%$ & $-1.5 \%$ & $-9.7 \%$ & $-25.9 \%$ & $-0.4 \%$ & $-9.7 \%$ & $-10.4 \%$ \\
C6 & $1.3 \%$ & $-4.5 \%$ & $-3.8 \%$ & $-10.6 \%$ & $-2.0 \%$ & $-7.8 \%$ & $-4.6 \%$ \\
C7 & $-4.4 \%$ & $-3.7 \%$ & $-24.2 \%$ & $-0.1 \%$ & $-11.0 \%$ & $-7.3 \%$ & $-8.4 \%$ \\
C8 & $-2.3 \%$ & $-16.7 \%$ & $-2.1 \%$ & $-7.1 \%$ & $-0.6 \%$ & $-3.3 \%$ & $-5.3 \%$ \\
C9 & $-0.9 \%$ & $2.3 \%$ & $-4.4 \%$ & $-11.0 \%$ & $4.7 \%$ & $-7.6 \%$ & $-2.8 \%$ \\
\hline Average & $-5.9 \%$ & $-8.6 \%$ & $-7.9 \%$ & $-8.6 \%$ & $-1.2 \%$ & $-7.5 \%$ & $-6.6 \%$ \\
\hline
\end{tabular}

On average, the ADP approach achieves $6.6 \%$ lower costs than the heuristic over all categories and instances, as seen in Table 3.3. To begin our analysis, we focus on the different categories (rows in Table 3.3). The savings range from $2.8 \%$ to $11.2 \%$. However, when we look into the performance of a category in the various instances, two observations stand out. First, in C4, the ADP approach achieves the largest cost reduction of all categories in all instances $\left(25.5 \%\right.$ in $\left.\mathrm{I}_{2}^{L}\right)$, as well as the largest average cost reduction among all instances (11.2\%). Second, in C9, the ADP approach achieves the worst performance 
of all categories in all instances (4.7\% higher costs than the heuristic in $\left.I_{5}^{L}\right)$, as well as the smallest average cost reduction among all instances $(2.8 \%)$. In these experiments we show that, although reductions are achieved in all categories on average over the test instances, a careful analysis must be done for some of the categories of the states the system can be in. We elaborate more on this difference in performance among categories in Section 3.5.

Focusing on the instances (columns in Table 3.3), we observe that, on average, cost reductions larger than $5.9 \%$ are achieved in all instances except $I_{5}^{L}$. Furthermore, we observe that in $\mathrm{I}_{5}^{L}$, the ADP approach generates higher costs than the heuristic in two categories (C3 and C9). In $\mathrm{I}_{1}^{L}$ and $\mathrm{I}_{2}^{L}$, the ADP policy generates higher costs than the heuristic in only one category ( $\mathrm{C} 6$ and $\mathrm{C} 9$, respectively). To study more into detail the pairwise differences, we calculate the $95 \%$ confidence intervals for all differences, as seen in Table 3.4. In most instances and categories, significant cost reductions are achieved. In addition to the two categories of $\mathrm{I}_{5}^{L}$ that had no savings on average (C3 and C9), we have two categories where the savings are not significant $(\mathrm{C} 1$ and $\mathrm{C} 2)$. We further elaborate on why the ADP policy under-performs in these cases, when compared to the others, in Section 3.5.

Table 3.4: Confidence intervals (at 95\%) of the difference between the benchmark policy and the ADP policy

\begin{tabular}{lcccccc}
\hline State & $\mathrm{I}_{1}^{L}$ & $\mathrm{I}_{2}^{L}$ & $\mathrm{I}_{3}^{L}$ & $\mathrm{I}_{4}^{L}$ & $\mathrm{I}_{5}^{L}$ & $\mathrm{I}_{6}^{L}$ \\
\hline C1 & {$[-7.0 \%,-4.8 \%]$} & {$[-9.6 \%,-7.5 \%]$} & {$[-10.3 \%,-8.4 \%]$} & {$[-6.1 \%,-4.9 \%]$} & {$[-1.3 \%, 0.0 \%]$} & {$[-5.9 \%,-4.5 \%]$} \\
C2 & {$[-9.7 \%,-8.4 \%]$} & {$[-13.1 \%,-11.6 \%]$} & {$[-4.8 \%,-3.3 \%]$} & {$[-3.6 \%,-1.8 \%]$} & {$[-1.2 \%, 0.1 \%]$} & {$[-11.6 \%,-10.4 \%]$} \\
C3 & {$[-2.7 \%,-1.2 \%]$} & {$[-7.2 \%,-6.1 \%]$} & {$[-9.1 \%,-7.4 \%]$} & {$[-3.8 \%,-2.4 \%]$} & {$[0.5 \%, 1.7 \%]$} & {$[-7.7 \%,,-6.7 \%]$} \\
C4 & {$[-16.0 \%,-13.8 \%]$} & {$[-26.5 \%,-24.6 \%]$} & {$[-6.2 \%,-4.1 \%]$} & {$[-12.5 \%,-11.2 \%]$} & {$[-2.2 \%,-0.7 \%]$} & {$[-8.4 \%,-7.6 \%]$} \\
C5 & {$[-15.9 \%,-14.3 \%]$} & {$[-2.0 \%,-0.9 \%]$} & {$[-10.5 \%,-8.8 \%]$} & {$[-26.5 \%,-25.3 \%]$} & {$[-1.0 \%, 0.1 \%]$} & {$[-10.3 \%,-9.2 \%]$} \\
C6 & {$[0.5 \%, 2.1 \%]$} & {$[-5.1 \%,-3.9 \%]$} & {$[-4.5 \%,-3.1 \%]$} & {$[-11.1 \%,-10.0 \%]$} & {$[-2.6 \%,-1.4 \%]$} & {$[-8.2 \%,-7.3 \%]$} \\
C7 & {$[-4.7 \%,-4.0 \%]$} & {$[-4.3 \%,-3.0 \%]$} & {$[-25.0 \%,-23.5 \%]$} & {$[-0.6 \%, 0.4 \%]$} & {$[-12.2 \%,-9.8 \%]$} & {$[-7.9 \%,-6.8 \%]$} \\
C8 & {$[-2.9 \%,-1.7 \%]$} & {$[-17.1 \%,-16.3 \%]$} & {$[-2.5 \%,-1.6 \%]$} & {$[-7.5 \%,-6.7 \%]$} & {$[-0.9 \%,-0.2 \%]$} & {$[-3.7 \% \%,-2.9 \%]$} \\
C9 & {$[-1.5 \%,-0.3 \%]$} & {$[1.8 \%, 2.8 \%]$} & {$[-5.4 \%,-3.5 \%]$} & {$[-11.4 \%,-10.7 \%]$} & {$[3.9 \%, 5.4 \%]$} & {$[-7.9 \%,-7.2 \%]$} \\
\hline
\end{tabular}

\subsection{Single-trip Interlude}

In this section, we compare the performance of our ADP algorithm against the benchmark heuristic in a simpler related problem: single-trip transport. As mentioned in Chapter 2 (Section 2.3.2), the single-trip problem considers only the delivery part, or in other words, the high-capacity mode departs from the origin and delivers freights to various destinations without returning to the origin. In the case of the LSP that motivated this problem, a single-trip would correspond to an external barge that is hired for half of the trip, meaning that once it delivers (or picks) the freight, the LSP is no longer responsible for the scheduling. We use a planning horizon of $T^{\max }=5$ and consider that there are no next-stage costs at the end of the horizon. We use two small and two large instances as seen in Table 3.5. The instances differ among themselves in the long-haul vehicle capacity, hence two values for $Q$ in Table 3.5. The first value of $Q$ in each instance type represents "normal" capacity, in comparison to the freights arriving per day, and the second one a "large" capacity. 
Table 3.5: Random variables in the numerical experiments

\begin{tabular}{lcc}
\hline Parameter & Small Instances & Large Instances \\
\hline $\mathcal{F}$ & $\{1,2\}$ & $\{1,2,3,4\}$ \\
$p_{f}^{\mathrm{F}}$ & $\{0.8,0.2\}$ & $\{0.25,0.25,0.25,0.25\}$ \\
$\mathcal{D}$ & $\{1,2,3\}$ & $\{1,2,3,4,5,6,7\}$ \\
$p_{d}^{\mathrm{D}, \mathrm{F}}$ & $\{0.1,0.8,0.1\}$ & $\{0.1,0.2,0.1,0.1,0.3,0.1,0.1\}$ \\
$\mathcal{R}$ & $\{0\}$ & $\{0,1,2\}$ \\
$p_{r}^{\mathrm{R}, \mathrm{F}}$ & $\{1\}$ & $\{0.3,0.3,0.4\}$ \\
$\mathcal{K}$ & $\{0,1,2\}$ & $\{0,1,2\}$ \\
$p_{k}^{\mathrm{K}, \mathrm{F}}$ & $\{0.2,0.3,0.5\}$ & {$[0.2,0.3,0.5\}$} \\
$C_{\mathcal{D}^{\prime}}$ & {$[250,1000]$} & {$[300,800]$} \\
$B_{d}$ & {$[500,1000]$} & $\{4,10\}$ \\
$Q$ & $\{2,5\}$ & \\
\hline
\end{tabular}

The parameters of the ADP algorithm are set as follows. The number of iterations is $N=2000$. We use the same set of features as in the round-trip, i.e., Features 3 . Feature weights are initialized with 1 for all features and all stages. The updating process $U^{V}$ is the same as for the round-trip case, i.e., the recursive least squares method for non-stationary data with double-pass procedure and iteration-dependent discount factor. For the comparison, we simulate the resulting policy of ADP and the benchmark heuristic for 2000 runs, using common random numbers for the comparison of arriving freights in the planning horizon.

For the small instances, we compare the optimal value of 20 initial states $S_{0}$ obtained with the Markov model against the value obtained with the simulation of the resulting policy of the ADP algorithm and the benchmark heuristic. The sample of states was chosen as follows. We looked at the solution value range of the Markov model and divided it into 20 segments from which a random state was chosen. This resulted in a sample of states containing freights with the three time-window lengths. Using these time-windows, we name the states from A to $\mathrm{T}$ as follows: State A has only freights with time-window length $k=2$ and State $\mathrm{T}$ has only freights with $k=0$ (i.e., urgent freights). The results for this comparison are shown in Table 3.6. For the large instances, we compare the performance of our ADP's policy against the heuristic. We test 12 random states fulfilling similar considerations as the sampled states of the small instances. The results are shown in Table 3.7.

In the small instance experiments shown in Table 3.6, we observe that the performance of our ADP's policy is, on average, 7.0\% away from optimal for the instance with $Q=2$ and $7.6 \%$ for the one with $Q=5$. The performance of the heuristic is $21.7 \%$ and $2.7 \%$ for the same instances, respectively. Furthermore, we observe a contrast in the performance between $\mathrm{ADP}$ and the heuristic. In the instance with $Q=2$, ADP outperforms the heuristic in 18 out of the 20 states. In the other instance, the exact opposite happens. With large capacity, the benchmark heuristic policy seems to be closer to optimal than the resulting policy of ADP. In the large instance experiments shown in Table 3.7, we observe that the performance of our ADP's policy is, on average, $8.3 \%$ better than the heuristic for the 
Chapter 3. Long-haul Round-trip Transport: Solution Analysis

Table 3.6: Policy performance for the small instances

\begin{tabular}{|c|c|c|c|c|c|c|c|c|c|c|}
\hline \multirow[b]{2}{*}{ State } & \multicolumn{5}{|c|}{$Q=2$} & \multicolumn{5}{|c|}{$Q=5$} \\
\hline & Markov M. & Heuristic & Diff. & $\mathrm{ADP}$ & Diff. & Markov M. & Heuristic & Diff. & ADP & Diff. \\
\hline Small A & 1182.3 & 1343.4 & $13.6 \%$ & 1330.0 & $12.5 \%$ & 1025.2 & 1052.5 & $2.7 \%$ & 1143.2 & $11.5 \%$ \\
\hline Small B & 1845.0 & 2887.8 & $56.5 \%$ & 1920.1 & $4.1 \%$ & 1110.5 & 1127.7 & $1.5 \%$ & 1224.1 & $10.2 \%$ \\
\hline Small C & 1351.9 & 2414.7 & $78.6 \%$ & 1465.6 & $8.4 \%$ & 940.3 & 960.9 & $2.2 \%$ & 1019.7 & $8.4 \%$ \\
\hline Small D & 2697.8 & 2773.6 & $2.8 \%$ & 3050.9 & $13.1 \%$ & 1475.2 & 1502.5 & $1.9 \%$ & 1593.2 & $8.0 \%$ \\
\hline Small E & 1508.0 & 1602.0 & $6.2 \%$ & 1597.3 & $5.9 \%$ & 1418.6 & 1433.1 & $1.0 \%$ & 1523.6 & $7.4 \%$ \\
\hline Small F & 2250.3 & 2990.0 & $32.9 \%$ & 3065.6 & $36.2 \%$ & 1692.1 & 1701.0 & $0.5 \%$ & 1791.1 & $5.9 \%$ \\
\hline Small G & 2908.0 & 3002.0 & $3.2 \%$ & 2997.3 & $3.1 \%$ & 1418.6 & 1433.1 & $1.0 \%$ & 1523.6 & $7.4 \%$ \\
\hline Small H & 2158.0 & 2252.0 & $4.4 \%$ & 2247.3 & $4.1 \%$ & 1068.6 & 1083.1 & $1.3 \%$ & 1173.6 & $9.8 \%$ \\
\hline Small I & 1058.0 & 1152.0 & $8.9 \%$ & 1147.3 & $8.4 \%$ & 968.6 & 983.1 & $1.5 \%$ & 1073.6 & $10.8 \%$ \\
\hline Small J & 1058.0 & 1152.0 & $8.9 \%$ & 1147.3 & $8.4 \%$ & 968.6 & 983.1 & $1.5 \%$ & 1073.6 & $10.8 \%$ \\
\hline Small K & 1758.0 & 2202.0 & $25.3 \%$ & 1847.3 & $5.1 \%$ & 1318.6 & 1333.1 & $1.1 \%$ & 1423.6 & $8.0 \%$ \\
\hline Small L & 1658.0 & 1802.0 & $8.7 \%$ & 1747.3 & $5.4 \%$ & 1568.6 & 1633.1 & $4.1 \%$ & 1673.6 & $6.7 \%$ \\
\hline Small M & 2008.0 & 2502.0 & $24.6 \%$ & 2097.3 & $4.4 \%$ & 1718.6 & 1733.1 & $0.8 \%$ & 1823.6 & $6.1 \%$ \\
\hline Small N & 2708.0 & 3202.0 & $18.2 \%$ & 2797.3 & $3.3 \%$ & 1718.6 & 1733.1 & $0.8 \%$ & 1823.6 & $6.1 \%$ \\
\hline Small O & 3408.0 & 3902.0 & $14.5 \%$ & 3497.3 & $2.6 \%$ & 1718.6 & 1733.1 & $0.8 \%$ & 1823.6 & $6.1 \%$ \\
\hline Small P & 2775.7 & 3122.7 & $12.5 \%$ & 2857.7 & $3.0 \%$ & 1718.6 & 1733.1 & $0.8 \%$ & 1823.6 & $6.1 \%$ \\
\hline Small Q & 2158.0 & 3152.0 & $46.1 \%$ & 2247.3 & $4.1 \%$ & 1618.6 & 1633.1 & $0.9 \%$ & 1723.6 & $6.5 \%$ \\
\hline Small R & 3158.0 & 4152.0 & $31.5 \%$ & 3247.3 & $2.8 \%$ & 1618.6 & 1633.1 & $0.9 \%$ & 1723.6 & $6.5 \%$ \\
\hline Small S & 3658.0 & 4652.0 & $27.2 \%$ & 3747.3 & $2.4 \%$ & 2118.6 & 2633.1 & $24.3 \%$ & 2223.6 & $5.0 \%$ \\
\hline Small T & 3508.0 & 3852.0 & $9.8 \%$ & 3597.3 & $2.5 \%$ & 2218.6 & 2333.1 & $5.2 \%$ & 2323.6 & $4.7 \%$ \\
\hline
\end{tabular}

Table 3.7: Policy performance for the large instances

\begin{tabular}{lcccccc}
\hline & \multicolumn{3}{c}{$Q=4$} & & $Q=10$ \\
\hline State & Heuristic & ADP & Difference & Heuristic & ADP & Difference \\
\hline Large A & 2962.9 & 2579.4 & $-12.9 \%$ & 1723.1 & 1743.0 & $1.2 \%$ \\
Large B & 9687.9 & 8729.4 & $-9.9 \%$ & 6448.1 & 5568.0 & $-13.6 \%$ \\
Large C & 5937.9 & 5579.4 & $-6.0 \%$ & 3223.1 & 2918.0 & $-9.5 \%$ \\
Large D & 1737.9 & 1754.4 & $1.0 \%$ & 1523.1 & 1543.0 & $1.3 \%$ \\
Large E & 2162.9 & 1804.4 & $-16.6 \%$ & 1523.1 & 1543.0 & $1.3 \%$ \\
Large F & 1362.9 & 1254.4 & $-8.0 \%$ & 848.1 & 868.0 & $2.3 \%$ \\
Large G & 1362.9 & 1254.4 & $-8.0 \%$ & 848.1 & 868.0 & $2.3 \%$ \\
Large H & 2187.9 & 2079.4 & $-5.0 \%$ & 1298.1 & 1318.0 & $1.5 \%$ \\
Large I & 3585.5 & 3550.0 & $-1.0 \%$ & 1766.3 & 1782.2 & $0.9 \%$ \\
Large J & 2537.9 & 2179.4 & $-14.1 \%$ & 1523.1 & 1543.0 & $1.3 \%$ \\
Large K & 3462.9 & 2979.4 & $-14.0 \%$ & 1123.1 & 1143.0 & $1.8 \%$ \\
Large L & 1778.1 & 1677.1 & $-5.7 \%$ & 1082.4 & 1101.2 & $1.7 \%$ \\
\hline
\end{tabular}

instance with $Q=4$ and $0.6 \%$ better for the one with $Q=10$. However, in the instance with $Q=10$, ADP outperforms the heuristic only in 2 out of the 12 states, while with $Q=4$, ADP outperforms the heuristic in all states.

Even though the tables above show performance differences among random, but carefully selected states, it is likely that in real-life problems, states and settings for which a look-ahead policy matters are more common than those for which it does not make a difference. For example, we observe that ADP brings an advantage in states with urgent freights and settings with restrictive capacities, which are common for LSPs. Furthermore, we observe that ADP performed better than the heuristic in normal capacity cases (i.e., where $Q$ equals the maximum number of arriving freights each day). However, in the large capacity 
cases (i.e., $Q$ is 2.5 times the smaller one), the heuristic seems to outperform the ADP. The intuitive reason for this is that, when there is too much capacity, postponing freights for future consolidation does not add any value. Freights "fit" at any decision moment, therefore it is reasonable that consolidating all freights that fit at the cheapest cost (i.e., exactly what the heuristic does) is, in this case, a near-optimal policy. Specifically in this small instance, the heuristic coincides with the optimal policy. When there is no effect of future costs in decisions, such as in the large capacity cases of our problem, look-ahead policies from ADP are simply not near-optimal [76]. Nevertheless, we observe that the value added by $\mathrm{ADP}$ with respect to the heuristic is substantial: on average, $10.3 \%$ better in the small instance with $Q=2$ and $8.3 \%$ better in the large instance with $Q=4$.

\subsection{Discussion}

Before applying our approach in practice, two aspects of our model should be brought to attention. First, all random variables in our model are defined as empirical distributions. These distributions can be derived from historical data. Furthermore, the "finite" nature of our model is in line with the constraints that hold in practice, such as the size of the container yard, maximum number of days a leased container can be used, etc. The finite time-window length means there is a maximum number of days that freight can be postponed. Second, the costs of a long-haul mode are modeled to depend on the subset of destinations visited rather than the route in which they are visited. The motivation for this choice comes from the destination characteristics of our problem: the Port of Rotterdam contains a limited number of container terminals. Thus, the optimal route for every combination of destinations can be calculated beforehand and used once the freights are selected. In addition, differences in waiting time, $\mathrm{CO}_{2}$ emissions, or other indicators of terminals, can be easily incorporated with the full definition of all subsets of destinations. In case there are many destinations in the region of the round-trip, these costs can be troubling to compute. To overcome this limitation in large networks, routing decisions should be incorporated into the problem. These decisions provide a new research opportunity on finding the balance between optimal routing and optimal postponing in large, stochastic, intermodal networks over a multi-period horizon.

As discussed in the previous chapter, it is possible that not all significant interactions among the stochastic variables are present in small instances used to design a "good" ADP solution for a problem. Without those interactions, results can differ in larger instances, which are common in practice. To illustrate that a smaller problem may not capture all relations of the larger problem, we apply the categorization procedure from Section 3.2.1 to Instance $\mathrm{I}_{2}^{S}$ from Chapter 2 and calculate descriptive statistics about the optimality gap of the ADP approach. We show these results in Table 3.8 and compare them with the performance of ADP in $\mathrm{I}_{2}^{L}$. These two instances have similar probability distributions but differ in size. The main conclusion from this comparison is that the differences in ADP performance among the different categories is much larger in the large instance $\mathrm{I}_{2}^{L}$ than in the small instance $\mathrm{I}_{2}^{S}$. Although this analysis is limited by our categorization of states, it supports the need for further research on the performance of $\mathrm{ADP}$ in different states of the same problem instance, for instance, to study how properties of states relate to the features. For example, a state with a low 
number of freights may require additional tuning of ADP parameters such as initial weights.

Table 3.8: Statistics about optimality gap in Instance $I_{2}^{S}$

\begin{tabular}{lllccccc}
\hline Category & Freights & Destinations & Min & Max & Average & $95^{\text {th }}$ Percentile & States \\
\hline C1 & Low & Low & $2.8 \%$ & $18.2 \%$ & $7.2 \%$ & $9.6 \%$ & 282 \\
C2 & Low & Medium & $2.5 \%$ & $39.8 \%$ & $7.5 \%$ & $15.5 \%$ & 2244 \\
C3 & Low & High & $1.9 \%$ & $31.5 \%$ & $7.5 \%$ & $17.9 \%$ & 1416 \\
C4 & Medium & Low & $2.8 \%$ & $26.4 \%$ & $6.5 \%$ & $10.2 \%$ & 150 \\
C5 & Medium & Medium & $2.0 \%$ & $36.2 \%$ & $6.9 \%$ & $16.0 \%$ & 2940 \\
C6 & Medium & High & $1.8 \%$ & $33.4 \%$ & $6.9 \%$ & $15.8 \%$ & 4008 \\
C7 & High & Low & $2.1 \%$ & $38.3 \%$ & $5.8 \%$ & $8.5 \%$ & 75 \\
C8 & High & Medium & $1.8 \%$ & $41.3 \%$ & $6.7 \%$ & $17.5 \%$ & 2550 \\
C9 & High & High & $1.5 \%$ & $36.5 \%$ & $6.4 \%$ & $15.6 \%$ & 5656 \\
\hline
\end{tabular}

The results from the previous section show that ADP performs better in some categories of states than others (see Table 3.3). States in these experiments represent initial conditions. As expected with the finite nature of our problem, some initial conditions, or categories of states, have a large impact on the performance of ADP over the entire horizon. In $\mathrm{C} 4$, which has a medium number of freights and a low number of destinations, the ADP approach performs the best. In C9, which has a large number of freights and a large number of destinations, the ADP approach performs the worst. These results are to be expected when considering the possible decisions in each of these two categories, with respect to the capacity of the high-capacity mode. In $\mathrm{C} 4$, which has a medium number of freights, it is possible to send a full barge, or a less than full barge. Naturally, these two actions differ in immediate costs, but also differ in future costs due to future consolidation possibilities. The $\mathrm{ADP}$ approach seems to capture better the future consolidation possibilities and therefore performs better over the entire horizon. In C9, which has a large number of freights, the alternative transport mode needs to be used frequently and postponement makes no sense. In this case, the daily costs will be large anyways, meaning that optimizing for each day individually as the heuristic does (i.e., no anticipation) coincides with the optimal policy. In other words, $\mathrm{ADP}$ and the heuristic have a similar performance. Investigating this, and other factors that influence the savings of ADP will lead to better designs. For example, we could use separate basis functions for different state categories of the same problem, thereby mixing a look-up table approach with the use of basis functions. Such differentiation of the state has been useful in single-mode stochastic transport problems [89].

In a broader view of performance, we also observed that the ADP approach performs well in 7 out of the 8 instances tested. Instances correspond to different problem settings, and could represent different LSPs. In $\mathrm{I}_{2}^{L}$ and $\mathrm{I}_{4}^{L}$, the ADP approach performs at least $8 \%$ better on average than the heuristic. $\mathrm{I}_{2}^{L}$ represents an "unbalanced" network, in terms of number of freights and destination probabilities, and $\mathrm{I}_{4}^{L}$ a network with many pre-announced orders. It seems that, when there are complex relations in the demand and when the planner knows about more orders in advance, the ADP approach particularly pays off. In $\mathrm{I}_{5}^{L}$, there is almost 
no difference between the ADP approach and the heuristic. $I_{5}^{L}$ represents an "urgent" network in which freights are mostly due on the day they are released, and they are mostly released the same day their information becomes known. In line with the observations from the last paragraph, in $\mathrm{I}_{5}^{L}$ there is less freedom on the decisions, and consequently lower benefits of using ADP in comparison to the heuristic. This analysis shows that we can establish up front whether ADP works better than the heuristic for a given problem setting. As Powell [76] points out, ADP performs well if the problem benefits from looking into the future.

A limitation of the single-period optimization benchmark used in this chapter may be its tendency to postpone the transport of freights. If there would be additional terminal constraints, such as operating schedules, fluctuating handling capacity, etc., the postponement decisions will be more restricted and the performance of ADP would get closer to that of the benchmark heuristic. In addition, postponement decisions may be less desirable when there are cost incentives to consolidate as much as possible. For example, in a problem setting where there high fixed (or setup) costs for any destination, with small differences among different destinations and low variable costs per freight, sending as many freights as possible is cost-efficient. In a way, this would be a single-period optimization method that "looks-ahead" by transporting freights that can be transported for very low costs today instead of tomorrow. To test for this, we performed a small sensitivity analysis using our large instances and a cost setup resembling the aforementioned situation (by setting $B_{d}=0$ ). In this analysis, the ADP approach achieves average savings of $3.1 \%$ instead of the $6.6 \%$ of the second phase experiments (Table 3.3). Although our sensitivity analysis is limited to a single cost parameter, it indicates how costs influence which decision method works best under stochastic multi-period problems. Further research is necessary with respect to both, the analysis of different cost structures, and the comparison over different look-ahead decision methods for various cost structures.

\subsection{Conclusions}

In this chapter, we studied the cost-reduction capabilities of our ADP approach compared to a benchmark heuristic under different problem settings. We showed that ADP results in significant savings compared to single-period optimization (between $5.9 \%$ and $8.6 \%$ on average on 7 out of the 8 instances tested). Moreover, we pointed out under which network settings ADP performs bests. As a managerial insight, companies with unbalanced demand and a large number of pre-announced orders can expect the largest savings when using ADP instead of single-period optimization.

Finally, we identified two areas for further research. The first area deals with differences in performance of our ADP approach across different initial states of the same problem instance. Investigating the causes of these differences can lead to improved ADP designs. The second area deals with the benefits that can be expected from the ADP approach in comparison to other benchmark heuristics. Research on which look-ahead approach is the most appropriate, considering additional criteria such as ease of implementation, can accelerate the adoption of look-ahead approaches in practice. Exploring these two areas will increase the value of decision methods that use probabilistic knowledge in transport and expand the knowledge on anticipatory decision-making in long-haul round-trips. 


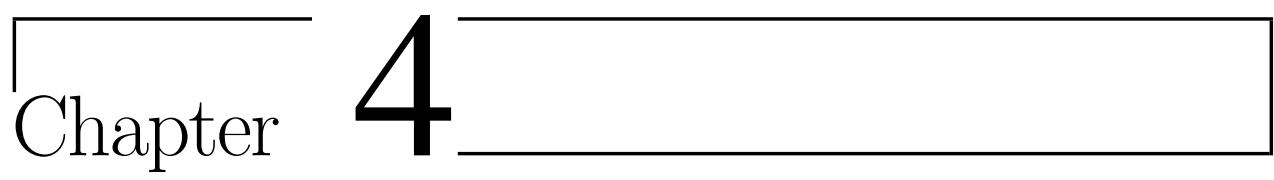

\section{Long-haul Multi-transfer Transport}

In this chapter, we consider the scheduling problem that LSPs face when transporting freights within their time-window in a long-haul network with multiple services and transfer terminals. Even though the LSP may have a transport plan from the origin to the destination of a freight, this plan can change at any decision moment given the actual circumstances. Even though freights are not known before they arrive, we assume there is probabilistic knowledge bout their arrival and their characteristics. The goal of the LSP is to maximize its profit over the entire network, considering that revenue and costs occur throughout time. In a similar way to Chapter 2, we model this problem as a Markov decision process and propose a heuristic solution based on Approximate Dynamic Programming (ADP). Due to the multi-period nature of the problem, the one-step look-ahead perspective of traditional ADP designs can make the heuristic flounder and end in a local-optimum. To tackle this, we study the inclusion of Bayesian exploration using the Value of Perfect Information (VPI) concept. In a series of numerical experiments, we show how VPI significantly improves a traditional ADP algorithm. Furthermore, we discuss our experience with merging VPI into ADP. This chapter is based on our publications in [70, 72].

\subsection{Introduction}

To move from multi-modal transport towards synchromodality, LSPs must employ the available services synchronously, to bring freights to their destination at agreed conditions such as time, costs, emissions, etc. [108]. In this chapter, we study how to schedule freight transport using the available services and transfers of a multi-modal network in synchromodality. A service refers to a transport mode with specific attributes such as 
schedule, duration, capacity, cost, etc. A transfer is the change from one service to another in a terminal. The flexibility of using any service and transfers for any freight allows LSPs to select, or to change, the next part of a freight's route as late as possible, with the possibility of including the latest information about the transport network. As a result, there are more consolidation options throughout the network and throughout time than in traditional multi-modal transport. However, identifying which of these consolidation opportunities are good is more challenging than in multi-modal transport.

To exemplify the consolidation opportunities and their complexity in synchromodal transport, consider a freight that has to be transported from 1 to 10 in Figure 4.1. This freight can be transported using 24 combinations of services across the seven terminals. In traditional multi-modal transport, freights usually have a pre-defined mode. If this would be the case for our freight, say barge only, then only 3 out of the 24 combinations would be feasible. Moreover, in multi-modal transport, freights usually require a fixed plan at the moment they are picked up at their origin. If this would be the case for our freight, say using the barge from 4 to 6 and subsequently from 6 to 8 , a last-minute change at 6 would not be possible. This change may be desirable, for instance, if there is an unforeseen delay between 6 and 8 , or when the train from 6 to 7 suddenly has cheaper space for transport upon arrival at 6 . Naturally, estimating whether a delay or cheaper space will occur at a future transfer in the network is challenging. However, looking ahead for these better consolidation opportunities can pay-off.

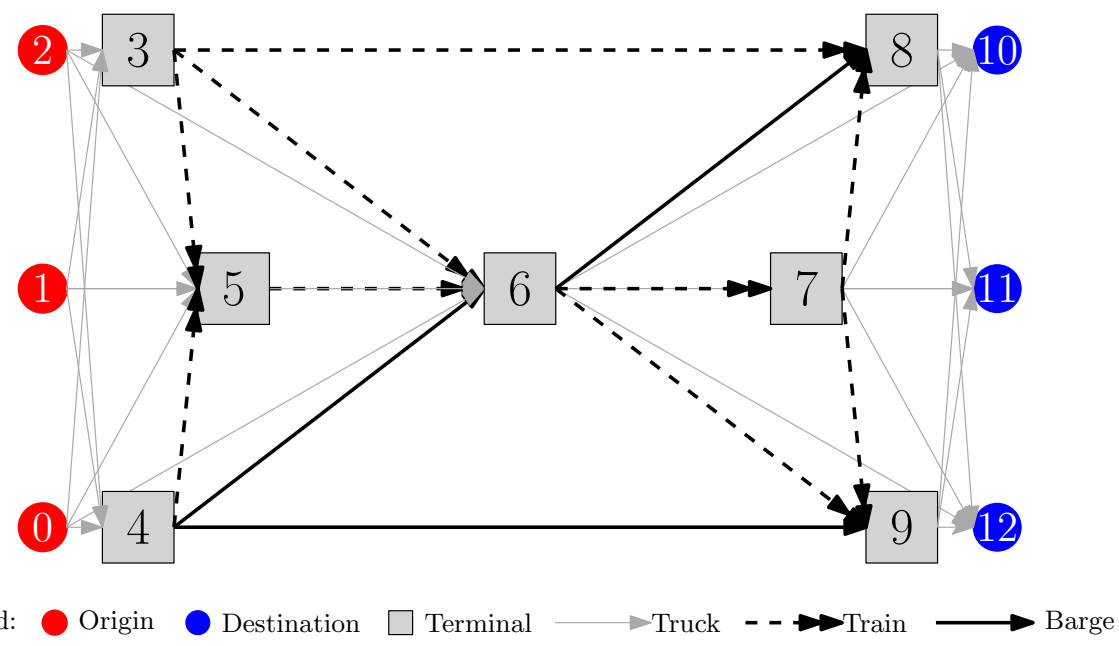

Figure 4.1: Example of a multi-modal network with seven terminals

In this chapter, we investigate scheduling methods that look ahead for consolidation opportunities in long-haul multi-transfer transport. The objective of our investigation is threefold: (i) to model the scheduling of freight in a long-haul multi-transfer transport network as a stochastic finite horizon optimization problem, (ii) to design a solution approach that handles 
the uncertainty and the time relations among parameters and variables in the scheduling problem, and (iii) to explore the use of our approach under various network configurations and freight demand patterns. The contribution of our work, following our objective, is threefold. First, we model the scheduling problem using a Markov Decision Process (MDP) model, which maximizes the expected reward over time. Second, we design a heuristic solution algorithm for the MDP model using the framework of Approximate Dynamic Programming (ADP) and incorporating Bayesian learning, using the concept Value of Perfect Information (VPI), to cope with the exploration versus exploitation tradeoff in ADP. Our algorithm iteratively estimates the impact of the scheduling options in immediate and posterior performance, and constructs a solution policy that anticipates on the stochastic freight demand and the network-wide performance over time. Third, we characterize how traditional ADP designs can make the algorithm flounder and end in a local-optimum and we analyze how VPI elements can help the algorithm to overcome those problems. More specifically, we describe, test, and show the benefits of various modifications to the VPI concept in ADP.

The remainder of this chapter is organized as follows. We begin by describing our problem, its characteristics and its challenges in Section 4.2. In Section 4.3, we examine relevant literature about scheduling synchromodal transport. We formulate an MDP model for our problem in Section 4.4. Subsequently, we present our approach to solve the model using ADP and VPI in Section 4.5. In Section 4.6, we test our approach under different network settings and discuss the results. In Section 4.7, we reflect upon the limitations of our approach. We finalize in Section 4.8 with our main conclusions and insights for further research.

\subsection{Problem Description}

We study the problem of scheduling freight in a long-haul multi-transfer transport network with the objective of maximizing performance over a multi-period horizon. In this problem, there are no restrictions on the services or transfers used to bring a freight to its destination and there are no fixed plans for freights. However, the destination, release-day and due-day of freights are fixed and known upon arrival of the freight. The number of freights that will arrive each period of the horizon and their characteristics are uncertain, but there is probabilistic information about their arrival (which may vary between periods of the horizon). On the supply side, we consider that all available services and transfers are fixed, but not necessarily the same, for each period of the horizon. We consider that a single network-wide LSP decides over all services and transfers even if they are not its own. We consider that the duration, cost, and revenue of any service may be spread over multiple periods in the horizon.

In general, the complexity of scheduling freight in a long-haul multi-transfer transport lies in the relation between the possible decisions, and their effect on the performance over time. At any given period of the horizon (which we refer to as day in the remainder of this chapter), there are three possibilities for scheduling a freight, independent of where it is located. The scheduler can either (i) transport a freight to its destination today, (ii) transport it to a multimodal terminal using a service available today, or (iii) postpone its transport to another day. Part of the impact of these scheduling options on the performance can be measured immediately (e.g., transport costs, revenue, holding costs, $\mathrm{CO}_{2}$ emissions, capacity utilization). How- 
ever, another part of their impact occurs on a posterior moment. For example, transporting a freight to its destination today reduces the number of freights to be considered for consolidation in the future; transporting a freight to a multi-modal terminal defines the future services that can be used for transporting it; and postponing a freight may reduce its feasible transport options due to its time-window, or may saturate the network. The future impact of each scheduling option is dependent on posterior decisions, as well as the freights that will arrive in the future and their characteristics (which are uncertain). It is therefore essential to estimate the impact of current scheduling decisions on the future performance, and to anticipate on it.

Before formulating a model and solution approach, we study the relevant literature about the characteristics and challenges of scheduling decisions in long-haul multi-transfer transport in the following section.

\subsection{Literature Review}

In this section, we review literature related to scheduling freight in long-haul multi-transfer transport. First, we take a look at scheduling problems that have explicitly been studied under synchromodal transport. Although this literature is scarce, and has only been published in the last three years, we review it in this chapter since it has been mostly done for transport networks similar to the one considered here. Subsequently, we focus our review on scheduling problems arising in dynamic and flexible inter/multi-modal transport. These problems can conceptually be seen as precursors for synchromodal transport. We briefly review the problem characteristics and proposed solutions of relevant multi-modal transport studies, and identify their shortcomings with respect to synchromodality. For an in-depth review of the scheduling problems arising in multi-modal transport, we refer the reader to [13], [92], and [56]. Afterwards, we briefly study literature using Approximate Dynamic Programming (ADP) for similar scheduling problems, a suitable approach for solving large-scale transport problems with stochastic freight arrivals, and discuss the necessary changes when applied to our problem. We finalize this section by stating our contribution to the literature related to scheduling long-haul, multi-transfer, and multi-modal transport.

Scheduling problems in synchromodality deal with flexibility in mode choice and with decisions based on real-time information [92]. These characteristics require that there is coordination between multiple network actors and an overview of transport supply and freight demand in the network for its scheduling [56], and that a balance between demand and supply is made every time new information becomes known [108]. Most studies specifically about synchromodal scheduling, focus on the flexibility aspect while somewhat ignoring the real-time information aspect. For example, Behdani et al. [5] and van Riessen et al. [109] determine the schedules of modes and the assignment of containers to the various modes assuming deterministic demand without incorporating the effect of real-time information and previous decisions in their models. In other words, they take a reactive approach and assume that re-planning can be done by solving the model again once the new information becomes known. Studies that explicitly consider re-planning (e.g., due to new demand or disruptions), such as Zhang and Pel [117] and [59], have taken a more proactive approach towards re-planning but not explicitly anticipate on future information. 
However, the studies that consider re-planning in multi-modal transport have shown that significant gains can be achieved with more proactive approaches.

Scheduling problems in multi-modal transport that are closely related to synchromodal transport can be categorized into two groups: (i) those that include dynamic and flexible assignment of freights to services and (ii) those that include anticipatory decisions on information that becomes known over time. A combination of these two groups constitutes scheduling synchromodal transport. In the first group, problems fall under the multi-modal scheduling family of Dynamic Service Network Design (DSND). In DSND methods, freights are assigned to transport services and modes in a network where least one feature varies over time [92]. Graph theory and (integer) linear programming methods are commonly used to model DSND problems due to their time-space nature. However, these methods have limitations for large and complex time-evolving problems [115], which are common to synchromodality [108]. To tackle these limitations, decomposition algorithms [36], receding horizons [51], and model predictive control [65], have been combined with DSND models in the literature. One disadvantage of combining these constructs with DSND models is that the relation between new stochastic information and the decisions is harder to include. This may explain why the majority of DSND studies considers deterministic demand only [92] although the need to incorporate uncertainty in demand has been recognized [52].

In the second group, most studies have been about extending DSND models with stochastic demand to anticipate on new information that becomes known over time. For example, [52] and [18] have used scenario generation methods to create schedules that are robust to the various demand realizations (i.e., new freights arriving). However, the resulting schedule does not adapt to new information dynamically as other methods do. Adapting dynamically means new schedules depend on the information that became know. Methods such as two-stage stochastic programming [53, 4] and ADP [22, 69], which include re-planning with the new information that became known, have been shown to have benefits over static decisions. However, when considering synchromodal scheduling, some limitations arise. In two-stage stochastic programming, explicit probabilistic formulations and computational complexity limit the size of problem instances that can be solved. In ADP, the design and validation of the approximation algorithm is problem specific. Nevertheless, ADP reduces the computational complexity while providing a close-to-optimal solution and has been shown to work well in the scheduling of freights in multi-modal transport $[70,71]$ and single-mode transport problems [86, 37, 107, 41]. Although ADP seems to be an ideal candidate to fill the gap in the literature about anticipatory scheduling of freight in long-haul multi-transfer transport, our problem requires more than its mere application.

In transport problems such as ours, the complex, time-revealing, and stochastic nature of the network makes the application of ADP difficult [80]. To begin with, the multi-period traveling times are a known issue to traditional ADP algorithms in transport problems [39]. Furthermore, when transport services have multiple attributes (in our case, different capacities, durations, costs, revenues, beginning and ending locations), the design of the Value Function Approximation (VFA) and its learning-phase play a crucial role in ADP [86]. In the design of the VFA, additional methods to the common post-decision state in ADP, such as aggregation of post-decision attributes [9] and sampling [40], may be necessary. 
In the learning phase of the VFA, a fundamental challenge that arises is the exploration versus exploitation problem [77]. The problem consists of whether to let ADP make the best decision according to its current estimate of the VFA (exploit) or let it make a different decision that may lead to an improvement of the VFA (explore).

The exploration versus exploitation dilemma has been widely studied in the reinforcement learning [96] and optimal learning communities [79]. The dilemma arises when a machine/agent tries to maximize its rewards by interacting with its environment through a series of actions. A widely studied optimization problem facing this dilemma is the so-called multi-armed bandit problem [55, 113]. Two approaches that have been applied to this problem, and the exploration versus exploitation dilemma, are evolutionary algorithms [110] and Bayesian learning methods [55, 94]. Bayesian learning methods usually rely on the concept of value of information [23], which appears under different names, among which expected improvement and knowledge gradient [85]. Although many real-life problems can be modeled as multi-armed bandit problems or solved using evolutionary algorithms and Bayesian learning methods, there are several difficulties to translate these approaches to a transport problem and to an ADP method as a solution approach. Among those difficulties we find the so-called "physical" state, where decisions depend on the state of our physical resources, such as containers and barges, and the correlation of values of alternative decisions (e.g., economies of scale in adding more containers to a barge) [85]. These difficulties are incorporated by [85] assuming an infinite horizon, whereas our problem deals with a finite horizon. To the best of our knowledge, the application of Bayesian learning techniques within finite horizon ADP has never been studied before. Nevertheless, it has been acknowledged that ADP can benefit from a translation of the knowledge on Bayesian learning to deal with the exploration versus exploitation dilemma [77, 85].

Overall, we observed in the literature that DSND models and methods provide a useful base for anticipatory scheduling of long-haul multi-transfer transport with some additional work. We believe that our contribution to DSND methods and synchromodal scheduling has three focus points. First, we design an MDP model and solution method based on ADP that incorporates stochastic freight demand characteristics and complex time and performance evolution of the transport network. Second, we explore the use of new exploration strategies for ADP based on Bayesian exploration, and provide design and validation insights. Third, we compare our ADP approach against a benchmark heuristic, under different problem characteristics, and specify further research directions based on the insights.

\subsection{Mathematical Model}

In this section, we formulate the problem of scheduling freights in long-haul multi-transfer transport in synchromodality using a Markov Decision Process (MDP) model. An MDP model analyzes the performance over time as been partly under the control of the decision maker and partly random. We begin by introducing the notation and all required input parameters. Subsequently, we formulate the elements of the MDP model: stages, states, decisions, transitions, and objective function. Finally, we examine the relations between the various elements of our model and identify challenges that these relations bring for heuristic 
approaches to solve the model.

\subsubsection{Notation}

We consider a finite horizon $\mathcal{T}$ of $T^{\max }$ days, i.e., $\mathcal{T}=\left\{0,1,2, \ldots, T^{\max }-1\right\}$. Although we refer to a period in the horizon as a "day", it is important to note that time can be discretized in any arbitrary interval as long as all time-dependent parameters are measured in that same interval. Each day $t \in \mathcal{T}$, the transport network is represented by a directed graph $G_{t}=\left(\mathcal{N}_{t}, \mathcal{A}_{t}\right)$, as it is usually done in DSND models. Nodes $\mathcal{N}_{t}$ denote locations where services begin or end, and $\operatorname{arcs} \mathcal{A}_{t}$ denote the services running from one location to another. To ease the formulation, we categorize nodes into three types: (i) origin nodes $\mathcal{N}_{t}^{\mathrm{O}}$, (ii) destination nodes $\mathcal{N}_{t}^{\mathrm{D}}$, and (iii) multi-modal terminal nodes $\mathcal{N}_{t}^{\mathrm{I}}$, such that $\mathcal{N}_{t}=\mathcal{N}_{t}^{\mathrm{O}} \cup \mathcal{N}_{t}^{\mathrm{D}} \cup \mathcal{N}_{t}^{\mathrm{I}}$; and we index all nodes in $\mathcal{N}_{t}$ by $i, j$, and $d$. In this categorization, the sets of origin and destination nodes represent the possible starting and ending locations of freights, respectively, and are mutually exclusive with the set of multi-modal terminals nodes. This separation of nodes applies to our model, but not necessarily to our problem. We further elaborate on this assumption, and how to overcome it, in Appendix 4-A.

Each day $t$, new freights with different characteristics arrive to the network. Each freight that arrives has a known origin $i \in \mathcal{N}_{t}^{\mathrm{O}}$, destination $d \in \mathcal{N}_{t}^{\mathrm{D}}$, release-day $r \in \mathcal{R}_{t}$, and time-window $k \in \mathcal{K}_{t}$. The release-day of a freight counts the number of days in which a freight will be released after its arrival. The set $\mathcal{R}_{t}=\left\{0,1,2, \ldots, R_{t}^{\max }\right\}$ ranges from immediate release to $R_{t}^{\max }$ days before release. The time-window of a freight measures the number of days in which a freight must be at its destination after it has been released. The set $\mathcal{K}_{t}=\left\{0,1,2, \ldots, K_{t}^{\max }\right\}$ ranges from the same day a freight is released to $K_{t}^{\text {max }}$ days after it is released. Although freights are unknown before they arrive, there is probabilistic knowledge about their arrival in each origin $i \in \mathcal{N}_{t}^{\mathrm{O}}$. In between two consecutive days $t-1$ and $t$, for origin $i \in \mathcal{N}_{t}^{\mathrm{O}}$, a total of $f \in \mathbb{N}$ freights arrive with probability $p_{f, i, t}^{\mathrm{F}}$. A freight that arrives between days $t-1$ and $t$ in origin $i \in \mathcal{N}_{t}^{\mathrm{O}}$ has destination $d \in \mathcal{N}_{t}^{\mathrm{D}}$ with probability $p_{d, i, t}^{\mathrm{D}}$, release-day $r \in \mathcal{R}_{t}$ with probability $p_{r, i, t}^{\mathrm{R}}$, and time-window $k \in \mathcal{K}_{t}$ with probability $p_{k, i, t}^{\mathrm{K}}$.

In a similar fashion to the categorization of nodes, we categorize arcs into three types: (i) arcs between an origin and a multi-modal terminal node $\mathcal{A}_{t}^{\mathrm{O}}=\left\{(i, j) \mid i \in \mathcal{N}_{t}^{\mathrm{O}}\right.$ and $\left.j \in \mathcal{N}_{t}^{\mathrm{I}}\right\}$, (ii) arcs between two multi-modal terminal nodes $\mathcal{A}_{t}^{\mathrm{I}}=\left\{(i, j) \mid i, j \in \mathcal{N}_{t}^{\mathrm{I}}\right\}$, and (iii) arcs between an origin or a multi-modal node, and a destination $\mathcal{A}_{t}^{\mathrm{D}}=\left\{(i, d) \mid i \in \mathcal{N}_{t}^{\mathrm{O}} \cup \mathcal{N}_{t}^{\mathrm{I}}\right.$ and $\left.d \in \mathcal{N}_{t}^{\mathrm{D}}\right\}$. For services beginning at an origin or ending at a destination (i.e., $\mathcal{A}_{t}^{\mathrm{O}}$ and $\mathcal{A}_{t}^{\mathrm{D}}$ ), we assume there is unlimited capacity every day. In other words, we assume that the preand end-haulage operations of our transport network are not restrictive. Typically, LSPs use trucks for their pre- and end-haulage operations and are able to hire additional trucks if required. However, for each service between two multi-modal terminals (i.e., $\mathcal{A}_{t}^{\mathrm{I}}$ ), we consider there is a maximum capacity of $Q_{i, j, t}$ freights and a transport duration of $L_{i, j, t}^{\mathrm{A}}$ days. In addition to the duration and capacity of each service, we consider that for each location $i \in \mathcal{N}_{t}$, there is a transfer duration of $L_{i, t}^{\mathrm{N}}$ days, which includes all handling operations, and we assume unlimited handling capacity. Consequently, the total time required for the service between two locations $M_{i, j, t}=L_{i, t}^{\mathrm{N}}+L_{i, j, t}^{\mathrm{A}}+L_{j, t}^{\mathrm{N}}$. Finally, we consider that between 
any two nodes, there is at most one arc (i.e., one service between two locations) and that all service durations are at least one (i.e., $M_{i, j, t} \geq 1 \forall(i, j) \in \mathcal{A}_{t}$ ). To represent more services between two locations, nodes and services can be modified as explained in Appendix 4-A.

With respect to the objective, we define a generic reward function $R_{t}(\cdot)$ to capture the immediate reward (i.e., reward at day $t$ ) of transporting freight in the network. For each arc $(i, j) \in \mathcal{A}_{t}$, or service between $i$ and $j$, we include three components in the reward function: (i) a revenue $A_{i, j, d, t}$ per freight with destination $d$, (ii) a setup cost $B_{i, j, t}$ independent of the number of freights using the service, and (iii) a variable cost $C_{i, j, d, t}$ per freight with destination $d$. These components can also have a value of zero to model different financial conditions such as receiving the entire revenue of a freight at the beginning of transport (i.e., $A_{i, j, d, t}=0 \forall i \notin \mathcal{N}_{t}^{\mathrm{O}}$ ) or constant cost for reserved space in a service (i.e., $B_{i, j, t}>0$ and $C_{i, j, d, t}=0$ for the reserved service $\left.(i, j)\right)$. Although various optimization criteria can be modeled using these three components, we note that the reward function $R_{t}(\cdot)$ is not limited to them and can other key performance indicators.

\subsubsection{Formulation}

The stages at which decisions are made in our MDP model correspond to the days in the horizon, i.e., $t \in \mathcal{T}$. The state of the system $S_{t} \in \mathcal{S}$ is modeled as the vector of all freights, and their characteristics, that are present at each node and each arc of the network (i.e., freights available at a location or traveling to a location) at stage $t$. In the state vector, we denote freights at location $i \in \mathcal{N}_{t}^{\mathrm{O}} \cup \mathcal{N}_{t}^{\mathrm{I}}$, that have destination $d \in \mathcal{N}_{t}^{\mathrm{D}}$, release-day $r \in \mathcal{R}_{t}^{\prime}$, and time-window $k \in \mathcal{K}_{t}$ with the integer variable $F_{i, d, r, k, t}$. Hence, the state is given by (4.1).

$$
S_{t}=\left[F_{i, d, r, k, t}\right]_{\forall i \in \mathcal{N}_{t}^{\mathrm{O}} \cup \mathcal{N}_{t}^{\mathrm{I}}, d \in \mathcal{N}_{t}^{\mathrm{D}}, r \in \mathcal{R}_{t}^{\prime}, k \in \mathcal{K}_{t}}
$$

Note that we use a different set $\mathcal{R}_{t}^{\prime}$ for the release-day $r$ to model freights that are being transported to location $i$ using $F_{i, d, r, k, t}$ in a computationally efficient way. We define $\mathcal{R}_{t}^{\prime}=$ $\left\{0,1,2, \ldots, \max \left\{R_{t}^{\max }, \max _{(i, j) \in \mathcal{A}_{t}^{\mathrm{I}}} M_{i, j, t}\right\}\right\}$ and use a virtual time-window to model freights that are en route. For example, if a freight is being transported to location $i$ using a service that departs on Monday and arrives at $i$ on Thursday, then this freight will be modeled in the state of Tuesday as a freight that will be available at $i$ in two days, i.e., a freight with releaseday $r=2$. Furthermore, if the freight has a deadline of Friday, its time-window on Tuesday will be $k=1$, i.e., one day after it arrives or, in terms of our virtual time-windows, it is virtually released. So, on Tuesday, instead of modeling the freight that is already released and being transported with $r=0$ and $k=4$, we model it with $r=2$ and $k=1$. We further elaborate on the use of virtual time-windows to capture the evolution of the network later on in this section.

At every stage, the planner decides which of the released freights (i.e., $r=0$ ) to transport using a given service and which ones to postpone. Remind that if the planner decides to transport a freight, only the part of a freight's route to the first destination is fixed, which may be a multi-modal terminal or its final destination. We model the decision with the vector $x_{t}$ consisting of all freights that will be transported at stage $t$, as shown in (4.2a). We denote the number of freights that will be transported from location $i$ to location $j$ 
(i.e., using service $(i, j) \in \mathcal{A}_{t}$ ), that have destination $d \in \mathcal{N}_{t}^{\mathrm{D}}$, and time-window $k \in \mathcal{K}_{t}$, with the integer variable $x_{i, j, d, k, t}$. Naturally, the decision $x_{t}$ is bounded by the feasible decision space $\mathcal{X}_{t}$ described by constraints $(4.2 \mathrm{~b})$ to $(4.2 \mathrm{f})$.

$$
\begin{gathered}
x_{t}=\left[x_{i, j, d, k, t}\right]_{\forall(i, j) \in \mathcal{A}_{t}, d \in \mathcal{N}_{t}^{\mathrm{D}}, k \in \mathcal{K}_{t}} \\
\text { s.t. } \\
\sum_{j \in \mathcal{N}_{t}^{\mathrm{I}} \cup\{d\}} x_{i, j, d, k, t} \leq F_{i, d, 0, k, t}, \quad \forall i \in \mathcal{N}_{t}^{\mathrm{O}} \cup \mathcal{N}_{t}^{\mathrm{I}}, d \in \mathcal{N}_{t}^{\mathrm{D}}, k \in \mathcal{K}_{t} \\
x_{i, d, d, L_{i, d, t}^{A}, t} \geq F_{i, d, 0, L_{i, d, t}^{A}, t}, \quad \forall(i, d) \in \mathcal{A}_{t}^{\mathrm{D}}, k \in \mathcal{K}_{t} \\
x_{i, j, d, k, t}=0, \quad \forall(i, j) \in \mathcal{A}_{t}^{\mathrm{O}} \cup \mathcal{A}_{t}^{\mathrm{I}}, d \in \mathcal{N}_{t}^{\mathrm{D}}, k \in \mathcal{K}_{t} \mid k<M_{i, j, t}+\widetilde{M}_{j, d, t} \\
\sum_{d \in \mathcal{N}_{t}^{\mathrm{D}}} \sum_{k \in \mathcal{K}_{t}} x_{i, j, d, k, t} \leq Q_{i, j, t}, \quad \forall(i, j) \in \mathcal{A}_{t}^{\mathrm{I}} \\
x_{i, j, d, k, t} \in \mathbb{N} \cup\{0\}, \quad \forall(i, j) \in \mathcal{A}_{t}, d \in \mathcal{N}_{t}^{\mathrm{D}}, k \in \mathcal{K}_{t}
\end{gathered}
$$

Constraints $(4.2 \mathrm{~b})$ ensure that, for every origin and multi-modal terminal, only freights that are released can be transported. Constraints (4.2c) guarantee that freights whose timewindow is as long as the duration of direct transport (i.e., trucking) are transported using this service. Note that with this constraint, we assume that trucking to a destination is faster than going via a multi-modal terminal, i.e., $L_{i, d, t}^{A}<\min _{j \in \mathcal{N}_{t}^{\mathrm{I}}}\left\{M_{i, j, t}+L_{j, d, t}^{A}\right\}, \forall(i, d) \in \mathcal{A}_{t}^{\mathrm{D}}$. Equations (4.2d) ensure that freights are not transported to a terminal $j$ if the fastest "multi-modal" route to their destination after arriving at that terminal (whose duration we denote with $\left.\widetilde{M}_{j, d, t}\right)$, is longer than the freight's time-window. This strict definition of transport options means that two trucking services cannot be used sequentially (e.g., bring a freight from its origin to a multi-modal terminal by truck and then transport it with truck from that multi-modal terminal to its destination). The value of $\widetilde{M}_{j, d, t}$ is case dependent: (i) if a freight is at an origin (i.e., $i \in \mathcal{N}_{t}^{\mathrm{O}}$ ), then $\widetilde{M}_{j, d, t}$ includes the duration of the shortest service from terminal $j$ to terminal $j^{\prime}$ and the duration of the trucking from $j^{\prime}$ to the destination $d$; (ii) if a freight is at a multimodal terminal (i.e., $i \in \mathcal{N}_{t}^{\mathrm{I}}$ ) then $\widetilde{M}_{j, d, t}=M_{j, d, t}$ since the multi-modal service $(i, j) \in \mathcal{A}_{t}^{\mathrm{I}}$ already accounts for the multi-modal part. Constraints (4.2e) ensure that the capacity of each service is not exceeded. Finally, constraints (4.2f) define the domain of the variables.

After making a decision $x_{t-1}$ and before entering the state $S_{t}$, exogenous information on new freights arrives. We denote the number of new freights with origin $i \in \mathcal{N}_{t}^{\mathrm{O}}$, destination $d \in \mathcal{N}_{t}^{\mathrm{D}}$, release-day $r \in \mathcal{R}_{t}$, and time-window $k \in \mathcal{K}_{t}$ that arrive in between two consecutive stages $t-1$ and $t$ with the integer variable $\widetilde{F}_{i, d, r, k, t}$. Hence, we model this exogenous information with the vector $W_{t}$, as seen in (4.3).

$$
W_{t}=\left[\widetilde{F}_{i, d, r, k, t}\right]_{\forall i \in \mathcal{N}_{t}^{\mathrm{O}}, d \in \mathcal{N}_{t}^{\mathrm{D}}, r \in \mathcal{R}_{t}, k \in \mathcal{K}_{t}}
$$

The transition from state $S_{t-1}$ to state $S_{t}$ depends on (i) the decision $x_{t-1}$, (ii) the exogenous information $W_{t}$, and (iii) the various time relations involving freights and services. 
We capture this transition using the function $S^{M}$, as seen in (4.4). First, and most naturally, decisions shift freights from one location to another through time. However, this shift can take longer than one stage, i.e., when a service duration spans more than one day. To avoid remembering decisions of services spanning more than one day (i.e., earlier decisions than $x_{t-1}$ ), we use the virtual time-windows. As exemplified before, virtual time-windows increase the release-day and reduce the time-window of freights that are transported using a service with a duration longer than one day, i.e., $M_{i, j, t}>1$. Second, the exogenous information increases the number of freights of a certain type that are present in the network. Third, various time relations apply to different types of freight. For example, released freights that are not transported remain at the same location and their time-window decreases. To capture all these relations, $S^{M}$ categorizes freight variables $F_{t, i, d, r, k}$ into seven equations, as shown in $(4.4 \mathrm{~b})$ to $(4.4 \mathrm{~h})$. We now elaborate on each category specifically.

$$
\begin{aligned}
& S_{t}=S^{M}\left(S_{t-1}, x_{t-1}, W_{t}\right) \\
& \text { s.t. } \\
& F_{i, d, 0, k, t}=F_{i, d, 0, k+1, t-1}-\sum_{j \in \mathcal{A}_{t}} x_{i, j, d, k+1, t-1}+F_{i, d, 1, k, t-1}+\widetilde{F}_{i, d, 0, k, t}, \\
& \forall i \in \mathcal{N}_{t}^{\mathrm{O}}, d \in \mathcal{N}_{t}^{\mathrm{D}}, k+1 \in \mathcal{K}_{t} \\
& F_{i, d, 0, K_{t}^{\max }, t}=F_{i, d, 1, K_{t}^{\max }, t-1}+\widetilde{F}_{i, d, 0, K_{t}^{\max }, t}, \\
& \forall i \in \mathcal{N}_{t}^{\mathrm{O}}, d \in \mathcal{N}_{t}^{\mathrm{D}} \\
& F_{i, d, 0, k, t}=F_{i, d, 0, k+1, t-1}-\sum_{j \in \mathcal{A}_{t}} x_{i, j, d, k+1, t-1}+F_{i, d, 1, k, t-1}+\sum_{j \in \mathcal{A}_{t} \mid M_{j, i, t}=1} x_{j, i, d, k+M_{j, i, t}, t-1}, \\
& \forall i \in \mathcal{N}_{t}^{\mathrm{I}}, d \in \mathcal{N}_{t}^{\mathrm{D}}, k+1 \in \mathcal{K}_{t} \\
& F_{i, d, r, k, t}=F_{i, d, r+1, k, t-1}+\widetilde{F}_{i, d, r, k, t}, \\
& \forall i \in \mathcal{N}_{t}^{\mathrm{O}}, d \in \mathcal{N}_{t}^{\mathrm{D}}, r+1 \in \mathcal{R}_{t} \mid r \geq 1, k \in \mathcal{K}_{t} \\
& F_{i, d, R_{t}^{\max }, k, t}=\widetilde{F}_{i, d, R_{t}^{\max }, k, t}, \\
& \forall i \in \mathcal{N}_{t}^{\mathrm{O}}, d \in \mathcal{N}_{t}^{\mathrm{D}}, k \in \mathcal{K}_{t} \\
& F_{i, d, r, k, t}=F_{i, d, r+1, k, t-1}+\sum_{j \in \mathcal{A}_{t} \mid M_{j, i, t}=r+1} x_{j, i, d, k+M_{j, i, t}, t-1} \text {, } \\
& \forall i \in \mathcal{N}_{t}^{\mathrm{I}}, d \in \mathcal{N}_{t}^{\mathrm{D}}, r+1 \in \mathcal{R}_{t}^{\prime} \mid r \geq 1, k \in \mathcal{K}_{t} \\
& F_{i, d,\left|\mathcal{R}_{t}^{\prime}\right|, k, t}=\sum_{j \in \mathcal{A}_{t}\left|M_{j, i, t}=\right| \mathcal{R}_{t}^{\prime} \mid+1} x_{j, i, d, k+M_{j, i, t}, t-1}, \\
& \forall i \in \mathcal{N}_{t}^{\mathrm{I}}, d \in \mathcal{N}_{t}^{\mathrm{D}}, k \in \mathcal{K}_{t}
\end{aligned}
$$

Equations (4.4b) define freights that are released (i.e., $r=0$ ), at an origin (i.e, $i \in \mathcal{N}_{t}^{\mathrm{O}}$ ), and with a time-window smaller than $K_{t}^{\max }$ (i.e., $k+1 \in \mathcal{K}_{t}$ ), as the sum of two types 
of freights from the previous stage at that origin with the same destination: (i) freights with a time-window of one stage longer that were not transported (i.e., $F_{t-1, i, d, 0, k+1}-$ $\sum_{j \in \mathcal{A}_{t}} x_{t-1, i, j, d, k+1}$ ), (ii) freights that had a release-day of one (i.e., $F_{t-1, i, d, 1, k}$ ) meaning that they would be released at the current stage, in addition to the new arriving freights in between the stages that had the same characteristics (i.e., $\widetilde{F}_{t, i, d, 0, k}$ ). Equations (4.4c) define freights that are released, at an origin, and have the maximum time-window as the sum of freights with a release-day of one and the new arriving freights with the same characteristics. Equations (4.4d) define freights that are released, at a multi-modal terminal, and with a time-window smaller than the maximum one, as the result of three types of freights from the previous stage at that terminal with the same destination: (i) freights with a time-window of one stage longer that were not transported, (ii) freights that had a release-day of one, and (iii) inbound freights from all locations $j$ whose service duration was one period (i.e., $\left.M_{j, i, t}=1\right)$ and whose time-window was the current freight's time-window plus the service duration (i.e., a reduced time-window from $k+M_{j, i, t}$ to $k$ ) at the moment of the decision $x_{t-1}$ (i.e., $\sum_{j \in \mathcal{A}_{t} \mid M_{j, i, t}=1} x_{t-1, j, i, d, k+M_{j, i, t}}$ ). Remind that, due to our separation of nodes, no new arriving freights come to multi-modal terminals and that, due to our assumption of a minimum of one day service duration, no freights at multi-modal terminals can have the maximum time-window. Equations (4.4e) define freights at an origin node that are still not released and do not have the maximum release-day, as the sum of two types of freight from the previous stage at that origin with the same destination and time-window: (i) freights with a release-day of one period longer than the current freight's release-day, and (ii) new arriving freights that had the same characteristics. Freights of the previous type that have the maximum release-day are the result of only the new arriving freights with the same characteristics, as shown in Equations (4.4f). Equations (4.4g) define freights that are at a multi-modal terminal and that are not released but do not have the maximum release-day, as the sum of two types of freight from the previous stage at that terminal: (i) freights with a release-day of one period longer than the current freight's release-day and the same timewindow, and (ii) freights sent in the decision of the previous stage, from all other locations to that terminal, whose service duration was equal to the release-day of the current freight plus one period and whose time-window was equal to the current freight's time-window plus the aforementioned service duration. Finally, freights at a multi-modal terminal with the maximum release-day of the virtual time-windows are the result of inbound freights to that location following the virtual time-windows reasoning, as shown in Equations (4.4h).

The immediate rewards of a decision $R_{t}\left(x_{t}\right)$ are calculated as shown in Equation (4.5). Remind that $A_{i, j, d, t}$ and $C_{i, j, d, t}$ are the revenue and variable cost of using service $(i, j) \in \mathcal{A}_{t}$ for one freight with destination $d$, respectively, and that $B_{i, j, t}$ is the setup cost for using the aforementioned service independent of the number of freights.

$$
\begin{aligned}
R_{t}\left(x_{t}\right)= & \sum_{(i, j) \in \mathcal{A}_{t}} \sum_{d \in \mathcal{N}_{t}^{\mathrm{D}}}\left(\left(A_{i, j, d, t}-C_{i, j, d, t}\right) \sum_{k \in \mathcal{K}_{t}} x_{i, j, d, k, t}\right) \\
& -\sum_{(i, j) \in \mathcal{A}_{t}}\left(B_{i, j, t} \cdot y_{i, j, t}\right)
\end{aligned}
$$


where

$$
y_{i, j, t}=\left\{\begin{array}{ll}
1, & \text { if } \sum_{d \in \mathcal{N}_{t}^{\mathrm{D}}} \sum_{k \in \mathcal{K}_{t}}\left(x_{i, j, d, k, t}\right)>0 \\
0, & \text { otherwise }
\end{array}, \forall(i, j) \in \mathcal{A}_{t}\right.
$$

Since the decision $x_{t}$ depends on the state, and the state is partially random, the objective is to find a policy that maximizes the expected discounted reward over the planning horizon. We denote a policy with $\pi \in \Pi$, and define it as a function that determines a decision $x_{t}^{\pi} \in \mathcal{X}_{t}$ for each possible state $S_{t} \in \mathcal{S}$. Thus, the objective can be expressed as shown in (4.6), where $\gamma_{t}$ is the discount factor balancing the impact of future and present rewards and $S_{0}$ is the initial state of the system.

$$
\max _{\pi \in \Pi} \mathbb{E}\left[\sum_{t \in \mathcal{T}} \gamma_{t} R_{t}\left(x_{t}^{\pi}\right) \mid S_{0}\right]
$$

Finite horizon MDP models such as ours can be solved using linear programming or dynamic programming. To design our solution approach, we focus on the latter. In dynamic programming, the optimal expected rewards can be estimated using a set of recursive equations and Bellman's principle of optimality, as shown in Equations (4.7). These equations can be solved backwards, from the end of the horizon towards the beginning. In Equations (4.7a), the state $S_{t+1}$ is partially random and partially the result of decision $x_{t}$. Using the transition function $S^{M}$, we can express $S_{t+1}$ as a function of the current state, decision, and a realization of the exogenous information, as shown in Equations (4.7b). Assuming that the possible realizations of the exogenous information $\Omega_{t+1}$ (i.e., $\left.W_{t+1} \in \Omega_{t+1}\right)$ are finite, and defining $p_{\omega}^{\Omega_{t+1}}$ as the probability of realization $\omega \in \Omega_{t+1}$, we can recursively solve our MDP as shown in Equations (4.7c). The solution to these last equations yield the optimal policy $\pi^{*}$. However, solving these equations is challenging for various reasons. In the following, we elaborate further on those solution challenges.

$$
\begin{gathered}
V_{t}\left(S_{t}\right)=\max _{x_{t} \in \mathcal{X}_{t}}\left(R_{t}\left(x_{t}\right)+\gamma_{t} \mathbb{E}\left[V_{t+1}\left(S_{t+1}\right)\right]\right), \quad \forall S_{t} \in \mathcal{S} \\
V_{t}\left(S_{t}\right)=\max _{x_{t} \in \mathcal{X}_{t}}\left(R_{t}\left(x_{t}\right)+\gamma_{t} \mathbb{E}\left[V_{t+1}\left(S^{M}\left(S_{t}, x_{t}, W_{t+1}\right)\right)\right]\right), \quad \forall S_{t} \in \mathcal{S} \\
V_{t}\left(S_{t}\right)=\max _{x_{t} \in \mathcal{X}_{t}}\left(R_{t}\left(x_{t}\right)+\gamma_{t} \sum_{\omega \in \Omega_{t+1}} p_{\omega}^{\Omega_{t+1}}\left(V_{t+1}\left(S^{M}\left(S_{t}, x_{t}, \omega\right)\right)\right)\right), \quad \forall S_{t} \in \mathcal{S}
\end{gathered}
$$

\subsubsection{Solution Challenges}

As with most MDP models, ours suffers from the so-called three curses of dimensionality [77]. Equations (4.7c) hold for the entire state space $\mathcal{S}$, decision space $\mathcal{X}_{t}$, and exogenous information space $\Omega_{t}$, which grow larger with an increasing size of the transport network and demand parameters. In addition to the curses of dimensionality, the assumption of a finite horizon in our MDP model brings additional challenges compared to an infinite horizon 
MDP. For example, in an infinite horizon MDP, the resulting policy is independent of the stage opposed to the finite horizon where it depends on the stage. In other words, infinite horizon MDPs yield one policy instead of $T^{\max }$ policies in the finite case. Even if we are only interested in the policy for the first decision moment in the finite case, we still need to learn the values for $|\mathcal{S}|$ states for $T^{\max }$ stages. However, the stationary-information assumption of the infinite horizon MDPs is not desired in our problem, where freight demand can have seasonality effects (e.g., different demand distributions on different stages) and services can change (e.g., increased capacity of a train, disruptions, etc.) over time. Although this stage-dependent information can be added to the state and transition definition in an infinite horizon, at the price of a larger state space, the finite horizon MDP allows us to start from the current state and use up-to-date information of what might happen in the finite period that lies ahead. In other words, it is easier to use the MDP for re-planning purposes once the information changes, which is a key characteristic of synchromodality.

Another solution challenge arises due to the interaction among the model's reward function, transition function, and the finite horizon; and the need for heuristic/approximation solution methodologies. In the freight transport industry, the revenue of a freight is usually received at a single point in time (e.g., at the pick-up or at the delivery) while the costs for the entire route are accrued in several points in time depending on the route. Although this is not an issue for the MDP model, it may result in strange behavior of heuristic approaches. When revenue is received at the pick-up, heuristics might prefer to pick up a freight as soon as possible, to receive the revenue, and then take it to the closest location and leave it there in order to avoid costs. In the opposite case, when the revenue is received at the delivery, heuristics might prefer to take a freight to its destination, as soon as possible, to receive the revenue, and avoid longer transport options which might be cheaper. These two behaviors accentuate more when considering the end-of-the-horizon effects. When revenue is received at the pick-up at the origin, heuristics might tend to postpone the transport of freight till the end of the horizon. When revenue is received at the delivery to the destination, heuristics might aim for an empty network at the end of the horizon. Consequently, the "greedy" nature of heuristics may lead to a poor performance in our finite horizon look-ahead model.

Although our MDP model brings various solution challenges, its components (e.g., transition function, decision constraints, exogenous information) can be used within the approximate dynamic programming framework to design a heuristic solution for them. We elaborate on this design, and how to overcome the challenges, in the following section.

\subsection{Solution Algorithm}

To solve the MDP model from the previous section, we use a heuristic approach based on the framework of Approximate Dynamic Programming (ADP). In ADP, the solution to the Bellman's Equations in (4.7) is approximated using simulation, along with other optimization and statistical techniques, in an iterative manner. In this section, we elaborate on two ADP designs. In Section 4.5.1, we describe a traditional design using basis functions and $\epsilon$-greedy exploration, that has worked in other multi-modal transport problems [71]. Furthermore, we comment on how the characteristics of our problem can make this traditional design flounder 
in apparent local-optima. In Section 4.5.2, we present ways to avoid these local-optima using ideas from Bayesian exploration and implementing them into a hybrid ADP design. Before elaborating on the traditional and hybrid ADP designs, we briefly describe the workings of the ADP algorithm. For an in-depth explanation of the ADP algorithm and its parts, we refer to [77].

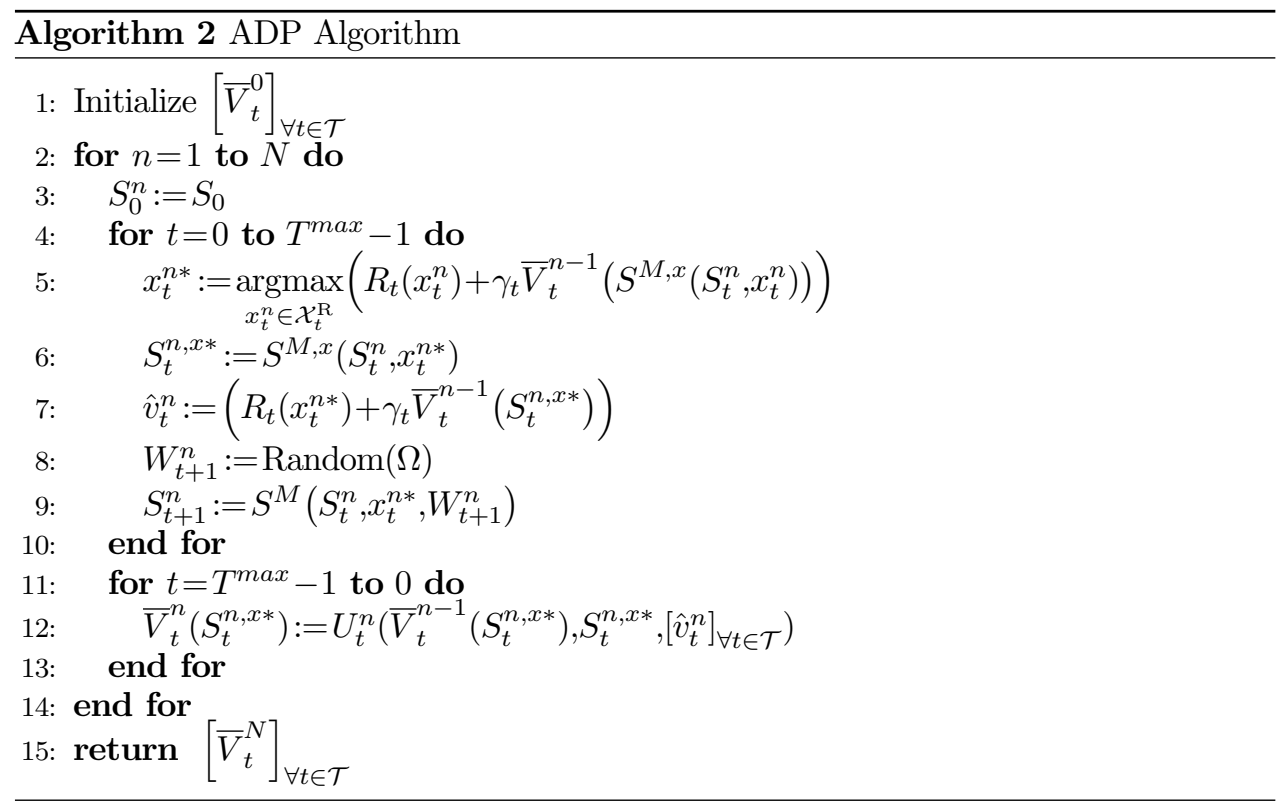

The ADP algorithm consists of various parts of the MDP model, such as the state, decision, and transition function definition, as shown in Algorithm 2. The ADP algorithm runs for a number of iterations $N$ and hence has all its variables indexed with $n$, as shown in Line 2. The overall idea of ADP is to replace the expectation of future rewards in (4.7a) with an approximation $\bar{V}_{t}^{n}$ and to update this approximation over the iterations. At the end, the algorithm yields the approximation of the last iteration, for all stages, as shown in Line 15. Thus, the output of ADP is a decision-making policy, based on the approximation $\left[\bar{V}_{t}^{N}\right]_{\forall t \in \mathcal{T}}$ of the expected rewards, in a similar way to the MDP.

The approximation $\bar{V}_{t}^{n}$ is a function of the so-called post-decision state $S_{t}^{x, n}$. The post-decision state is the state of the system after a decision has been made but before the new exogenous information becomes known and the next stage state is realized, i.e., $S_{t}^{n, x}=S^{M, x}\left(S_{t}^{n}, x_{t}^{n}\right)$. The transition $S^{M, x}$ to the post-decision state works in a similar way as the transition function $S^{M}$, see (4.4), with the only difference that no exogenous information is considered, i.e., all $\widetilde{F}_{i, d, r, k, t}$ variables are omitted in $(4.4 \mathrm{~b})$ to $(4.4 \mathrm{~h})$. To update the approximation $\bar{V}_{t}^{n}$, the algorithm simulates the use of its resulting policy for all 
stages. In contrast to backwards dynamic programming, ADP moves forward in the stages, as shown in Line 4. In each stage, the optimality equations in (4.7) are transformed into one single equation (using $\bar{V}_{t}^{n}$ ), as shown in Line 7 . Furthermore, the decision that attains the maximum reward $\hat{v}_{t}^{n}$, as well as its corresponding post-decision state, are stored as shown in Lines 5 and 6 . To advance to the next stage $t+1$, the algorithm uses a sample from $\Omega_{t+1}$, obtained through a Monte Carlo simulation, and the transition function $S^{M}$ defined in (4.4), as shown in Line 8. After all stages are processed, a function $U_{t-1}^{n}$ is used to update the approximation in a backward manner, as seen in Lines 11 to 13. This function uses the information stored throughout the stages. The entire procedure just described is then repeated $N$ times.

The benefit of having the approximation $\bar{V}_{t}^{n}$ in ADP is two-fold. First, it avoids enumerating all possible realizations of the exogenous information $\Omega_{t}$. Second, it allows the optimality equation in Line 7 to be solved for one state at a time, instead of for all states. These two benefits eliminate two of the curses of dimensionality. However, the large decision space must be tackled separately. For this, we propose the use of a Restricted Policy (RP). We design two RPs by adding more constraints to the feasible decision space $\mathcal{X}_{t}$ described by constraints $(4.2 \mathrm{~b})$ to $(4.2 \mathrm{f})$. All restrictions significantly reduce the number of feasible decisions to evaluate; some restrictions do so without harming solution quality whereas others limit the solution quality that ADP can attain. We elaborate more on this issue in Section 4.7. For now, we describe the two RPs.

The first RP, denoted by $\mathcal{X}_{t}^{\mathrm{RP} 1}$ and defined in (4.8), works with three additional constraints to the feasible decision space. First, freights that are not urgent (i.e., $k>L_{i, d, t}^{A}$ ) cannot use direct trucking to their destination (i.e., service $(i, d)$ ), as shown in Restriction (4.8b). Second, all freights that are at the same location and go to the same destination must be transported together, as shown in Restriction (4.8c). To achieve this, we use the binary variable $x_{i, j, d, t}^{\mathrm{RG}}$, which gets a value of 1 if freights at location $i$ with destination $d$ are sent using service $(i, j) \in \mathcal{A}_{t}^{\mathrm{I}}$ and 0 otherwise, and the binary parameter $M_{i, j, d, k, t}^{\mathrm{R}}$, which gets a value of 1 if the fastest multi-modal route for freights at location $i$ with destination $d$ and time-window $k$ is longer than the freight's time-window (i.e., $k<M_{i, j, t}+\widetilde{M}_{i, d, t}$ ). Third, we consider that all freights that arrive at an origin, independent of their characteristics, must be either transported to the same multi-modal terminal or postponed, as shown in Restriction (4.8d). To achieve this, we use the binary variable $x_{j, t}^{\mathrm{RO}}$, which gets a value of 1 if the chosen multi-modal terminal is $j$ and 0 otherwise. Naturally, we assume with this last restriction that there is at least one terminal to which freight from all origins can be brought to.

$$
\begin{gathered}
x_{t} \in \mathcal{X}_{t} \\
x_{i, d, d, k, t}=0, \quad \forall i \in \mathcal{N}_{t}^{\mathrm{O}} \cup \mathcal{N}_{t}^{\mathrm{I}}, d \in \mathcal{N}_{t}^{\mathrm{D}}, k \in \mathcal{K}_{t} \mid k>L_{i, d, t}^{A} \\
x_{i, j, d, k, t} \geq\left(F_{i, d, 0, k, t}\right)\left(x_{i, j, d, t}^{\mathrm{RG}}-M_{i, j, d, k, t}^{\mathrm{R}}\right), \\
\forall i \in \mathcal{N}_{t}^{\mathrm{O}} \cup \mathcal{N}_{t}^{\mathrm{I}}, j \in \mathcal{N}_{t}^{\mathrm{I}}, d \in \mathcal{N}_{t}^{\mathrm{D}}, k \in \mathcal{K}_{t} \\
\sum_{d \in \mathcal{N}_{t}^{\mathrm{D}}} \sum_{i \in \mathcal{N}_{t}^{\mathrm{O}}} x_{i, j, d, k, t}=\left|\mathcal{N}_{t}^{\mathrm{D}}\right|\left|\mathcal{N}_{t}^{\mathrm{O}}\right| x_{j, t}^{\mathrm{RO}}, \quad \forall j \in \mathcal{N}_{t}^{\mathrm{I}} \mid \exists_{\forall i \in \mathcal{N}_{t}^{\mathrm{O}}}(i, j) \in \mathcal{A}_{t}^{\mathrm{I}}
\end{gathered}
$$

The second RP, denoted by $\mathcal{X}_{t}^{\mathrm{RP} 2}$ and defined in (4.9), works similarly to RP1 with 
one difference. Instead of grouping all freights that arrive in all origins, as in (4.8d), we group all freights that arrive in all origins that go to the same destination, as seen in (4.9d). This means that all freight that arrives to any origin with a given destination must either be brought to the same terminal or postponed. To achieve this, we use the binary variable $x_{j, d, t}^{\mathrm{RO} 2}$, which gets a value of 1 if the multi-modal terminal $j$ is chosen for freights with destination $d$ and 0 otherwise. RP2 is computationally more expensive than RP1 but also gives more options to choose from at the pick-up of freight, possibly resulting in better solutions. We analyze the solution quality of both RPs in the Section 4.6. It is important to note that, if computational resources allow, these restricted policies can be omitted.

$$
\begin{gathered}
x_{t} \in \mathcal{X}_{t} \\
x_{i, d, d, k, t}=0, \quad \forall i \in \mathcal{N}_{t}^{\mathrm{O}} \cup \mathcal{N}_{t}^{\mathrm{I}}, d \in \mathcal{N}_{t}^{\mathrm{D}}, k \in \mathcal{K}_{t} \mid k>L_{i, d, t}^{A} \\
x_{i, j, d, k, t} \geq\left(F_{i, d, 0, k, t}\right)\left(x_{i, j, d, t}^{\mathrm{RG}}-M_{i, j, d, k, t}^{\mathrm{R}}\right), \\
\forall i \in \mathcal{N}_{t}^{\mathrm{O}} \cup \mathcal{N}_{t}^{\mathrm{I}}, j \in \mathcal{N}_{t}^{\mathrm{I}}, d \in \mathcal{N}_{t}^{\mathrm{D}}, k \in \mathcal{K}_{t} \\
\sum_{i \in \mathcal{N}_{t}^{\mathrm{O}}} x_{i, j, d, k, t}=\left|\mathcal{N}_{t}^{\mathrm{O}}\right| x_{j, d, t}^{\mathrm{RO} 2}, \quad \forall j \in \mathcal{N}_{t}^{\mathrm{I}} \mid \exists_{\forall i \in \mathcal{N}_{t}^{\mathrm{O}}}(i, j) \in \mathcal{A}_{t}^{\mathrm{I}}, d \in \mathcal{N}_{t}^{\mathrm{D}}
\end{gathered}
$$

\subsubsection{ADP with Basis Functions and Epsilon-Greedy Exploration}

In our first ADP design, we use basis functions for the approximation $\bar{V}_{t}^{n}\left(S_{t}^{x, n}\right)$. The overall idea of basis functions is to quantify characteristics of a post-decision state that explain the expected future rewards to a certain degree. We denote the basis function of a characteristic $b \in \mathcal{B}_{t}$ with $\phi_{b, t}: S_{t}^{x, n} \rightarrow \mathbb{R}$ and the degree (i.e., weight) with which it explains the future rewards by $\theta_{b, t}^{n} \in \mathbb{R}$. The approximated future rewards are the result of the product between all basis functions and their weights, as shown in (4.10). Using matrix notation, we define $\phi_{t}\left(S_{t}^{x, n}\right)=\left[\phi_{1, t}\left(S_{t}^{x, n}\right), \ldots, \phi_{\left|\mathcal{B}_{t}\right|, t}\left(S_{t}^{x, n}\right)\right]^{\mathrm{T}}$ as the column vector of basis functions, and $\theta_{t}^{n}=\left[\theta_{1, t}^{n}, \ldots, \theta_{|\mathcal{B}|, t}^{n}\right]^{\mathrm{T}}$ as the column vector of weights at iteration $n$ and stage $t$. We will use these vectors to describe the updating procedure later on.

$$
\bar{V}_{t}^{n}\left(S_{t}^{x, n}\right)=\sum_{b \in \mathcal{B}} \theta_{b, t}^{n} \phi_{b, t}\left(S_{t}^{x, n}\right)=\phi_{t}\left(S_{t}^{x, n}\right)^{\mathrm{T}} \theta_{t}^{n}
$$

At each stage $t$, the set of characteristics $\mathcal{B}_{t}$ counts two types of freights per locationdestination pair: (i) freights whose time-window is shorter than the duration of the shortest multi-modal path of the entire network, which we denote as $\psi$, as shown in (4.11a), and (ii) freights whose time-window is at least the duration of the shortest multi-modal path of the entire network, as shown in (4.11b). Furthermore, we also count the total number of freights going to each destination, independent of their current location, release-day, or time-window, as shown in (4.11c). Finally, we add a constant as shown in (4.11d). We have a total number of characteristics $\left|\mathcal{B}_{t}\right|=2\left(\left|\mathcal{N}_{t}^{\mathrm{O}}\right|\left|\mathcal{N}_{t}^{\mathrm{I}}\right|\right)+\left|\mathcal{N}_{t}^{\mathrm{D}}\right|+1$. To index them, we 
use the functions $b, b^{\prime \prime}$, and $b^{\prime \prime \prime}$, with range $\left[1, \ldots,\left|\mathcal{B}_{t}\right|\right]$.

$$
\begin{aligned}
\phi_{b(i, d), t}\left(S_{t}^{x, n}\right) & =\sum_{k \in \mathcal{K}_{t} \mid k<\Psi} \sum_{r \in \mathcal{R}_{t}^{\prime}} F_{i, d, r, k, t}^{x, n}, \quad \forall i \in \mathcal{N}_{t}^{\mathrm{O}} \cup \mathcal{N}_{t}^{\mathrm{I}}, d \in \mathcal{N}_{t}^{\mathrm{D}} \\
\phi_{b^{\prime}(i, d), t}\left(S_{t}^{x, n}\right) & =\sum_{k \in \mathcal{K}_{t} \mid k \geq \Psi r \in \mathcal{R}_{t}^{\prime}} \sum_{i, d, r, k, t}, \quad \forall i \in \mathcal{N}_{t}^{\mathrm{O}} \cup \mathcal{N}_{t}^{\mathrm{I}}, d \in \mathcal{N}_{t}^{\mathrm{D}} \\
\phi_{b^{\prime \prime}(d), t}\left(S_{t}^{x, n}\right) & =\sum_{i \in \mathcal{N}_{t}^{\mathrm{O}} \cup \mathcal{N}_{t}^{\mathrm{I}} k \in \mathcal{K}_{t} \mid k \geq \Psi r \in \mathcal{R}_{t}^{\prime}} \sum_{i, d, r, k, t}, \quad \forall d \in \mathcal{N}_{t}^{\mathrm{D}} \\
\phi_{\left|\mathcal{B}_{t}\right|}\left(S_{t}^{x, n}\right)=1 &
\end{aligned}
$$

The basis functions $\phi_{t}\left(S_{t}^{x, n}\right)$ are fixed for all iterations, changing value only with respect to the post-decision state $S_{t}^{x, n}$ and the network at stage $t$. The weights $\theta_{b, t}^{n}$, however, depend on an iteration $n$ and stage $t$. The idea is that, throughout the iterations, the observed rewards for each stage can be used to update the weights, and thus the approximation $\bar{V}_{t}^{n}\left(S_{t}^{x, n}\right)$. Similar to Chapter 2 (Section 2.4.2), we use the recursive least squares for non-stationary data method for the updating function $U_{t-1}^{n}$ as shown in (4.12). However, Equation (4.12) is a bit different (simpler) than Equation (2.16) in Chapter 2 because we already substituted for $\gamma^{n}$ in equations with the $B$ matrix, expressed everything in terms of the $H$ matrix, and substituted $\alpha^{n}$ for a parameterized function $\lambda^{n}$. This updating method still uses three variables to update the weights: (i) the difference between the next-stage estimate from the previous iteration $\bar{V}_{t-1}^{n-1}\left(S_{t-1}^{x, n}\right)$ and the observed reward $\hat{v}_{t}^{n}$ at the current iteration, (ii) the value of each basis function $\phi_{b}\left(S_{t}^{x, n}\right)$, and (iii) the optimization matrix $H^{n}$. This optimization matrix can put more emphasis on recent observations. This can be beneficial when in early iterations the approximation is far from optimal or when the initial conditions of the system may bias the approximation. For a comprehensive explanation on the recursive least squares method for non-stationary data we refer to [77].

$$
\begin{gathered}
\bar{V}_{t}^{n}\left(S_{t}^{n, x *}\right):=U_{t}^{n}\left(\bar{V}_{t}^{n-1}\left(S_{t}^{n, x *}\right), S_{t}^{n, x *},\left[\hat{v}_{t}^{n}\right]_{\forall t \in \mathcal{T}}\right) \\
\text { s.t. } \\
\theta_{t}^{n}=\theta_{t}^{n-1}-\left(H_{t}^{n}\right)^{-1} \phi_{t}\left(S_{t}^{x, n}\right)\left(\bar{V}_{t-1}^{n-1}\left(S_{t-1}^{x, n}\right)-\sum_{t}^{T^{\max }-1} \hat{v}_{t}^{n}\right) \\
H_{t}^{n}=\lambda^{n} H_{t}^{n-1}+\phi_{t}\left(S_{t}^{x, n}\right) \phi_{t}\left(S_{t}^{x, n}\right)^{\mathrm{T}} \\
\lambda^{n}=1-\frac{\lambda}{n}
\end{gathered}
$$

The ADP design presented before uses the so-called exploitation decision strategy. Exploitation decisions are those that take us to the best post-decision state given our estimate of the downstream rewards at that iteration, as seen in Line 5 of Algorithm 2. If these estimates are far from good, the post-decision state they take us too can also be far from optimal, and since the estimates are updated with the post-decision state we saw (i.e., using the basis functions), 
we might end-up in a chain reaction for the worse. A different approach to make decisions, which aims to avoid such cycles during the learning phase of ADP, is to consider exploration decisions. As their name states, exploration decisions are those that take us to post-decision states that we might not have seen before, even if they are not the best ones given. In analogy to local search heuristics, exploration decisions can be seen as moves that prevent ADP from getting stuck in local optima. The benefit of making exploration decisions in our basis functions approach is that we may observe post-decision state characteristics we had not seen before (i.e., basis function values different than zero), or not that often, and therefore improving the approximation of the downstream rewards. One way to consider exploration decisions is the $\epsilon$-greedy strategy [77]. In this strategy, a fraction $\epsilon$ of the decisions through the iterations should be exploration ones. In other words, a random decision from $\mathcal{X}_{t}^{\mathrm{R}}$ is chosen with probability $\epsilon$. To implement it, Line 5 in Algorithm 2 must be exchanged with Algorithm 3.

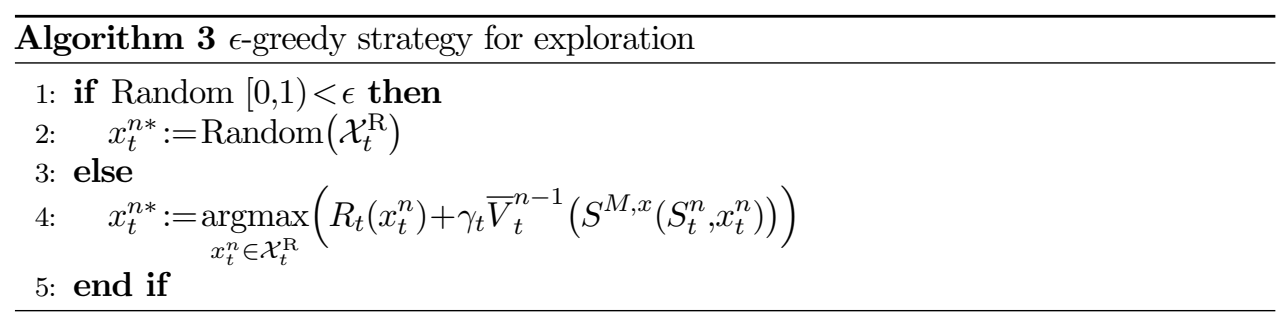

Although exploration decisions may take us to possibly better post-decision states, we also run the risk of deteriorating our approximation and thus making worse exploitation decisions. This is caused by updating the weights of basis functions that we have already seen (and for which we may have reasonable values) using values resulting from possibly far-from-optimal decisions. Although one could update the approximation using the exploitation decision rather than the exploration one (known as off-policy updating), in a finite horizon problem this results in a larger propagation of errors across the stages since the observed post-decision states (and hence the value of the basis functions we use for updating our approximation) depend on the exploration decision and not the exploitation one. In the following section, we introduce a different exploration strategy that aims to balance the trade-off between visiting relatively unknown post-decision states and the risk of deteriorating the current approximation.

\subsubsection{ADP with Bayesian Exploration}

In ADP, exploration decisions have the potential to improve our approximation when the approximation is not good. Exploitation decisions have the potential to improve our approximation further once the approximation is good. Since there is uncertainty on whether we already have a good approximation, the balance between exploration and exploitation decisions can be seen as a stochastic optimization sub-problem. A way to quantify the uncertainty and balance this tradeoff in ADP is through Bayesian exploration, using the concept of Value of Perfect Information (VPI), as proposed in Ryzhov et al. [85]. Although this technique has been recently applied successfully to infinite horizon problems, applying 
it to finite horizon problems such as ours comes with several challenges. As far as we know, this has not been considered before. In this section, we introduce the general idea of Bayesian exploration and subsequently present the challenges and possible modifications to apply it to finite horizon problems in what we call a hybrid ADP design. For in-depth explanation of Bayesian exploration we refer the reader to Powell and Ryzhov [79].

During the early iterations of the ADP algorithm, there is a lot of uncertainty about the approximation of the downstream rewards. As the algorithm progresses, and more post-decision states are observed, this uncertainty is reduced. However, if the same post-decision states are observed over and over again, there could be a bias towards these post-decision states just because we have good estimates about their downstream rewards (i.e., due to updating the corresponding basis function weights). The general idea of Bayesian exploration is to prevent such a bias in the algorithm by making decisions that will provide information about which post-decision states are actually better than the ones we thought were best. In contrast to the way of making decisions in $\epsilon$-greedy exploration, in Bayesian exploration we make the decision that provides the maximum value of exploration $v_{t}^{E, n}$ at stage $t$ and iteration $n$, as shown in (4.13). In (4.13), $K_{t}^{n}$ represents the knowledge about the uncertainty in the approximation of the downstream rewards. We now describe $v_{t}^{E, n}$ and $K_{t}^{n}$ in more detail.

$$
x_{t}^{n *}=\underset{x_{t}^{n} \in \mathcal{X}_{t}^{\mathrm{R}}}{\operatorname{argmax}}\left(v_{t}^{E, n}\left(K_{t}^{n}, S_{t}^{n} x_{t}^{n}\right)\right)
$$

In the VPI concept applied to ADP, the value of exploration $v_{t}^{E, n}$ of a decision $x_{t}^{n}$ is defined as the expected improvement in the approximated downstream reward that arises from visiting the post-decision state corresponding to $x_{t}^{n}$. It is defined as "expected" because the true value of the approximation is considered to be a random variable for which we have an initial distribution of belief [79]. The best estimate of the mean of this random variable at iteration $n$ and stage $t$ is $\bar{V}_{t}^{n}\left(S_{t}^{n, x}\right)$. Recall that in our basis function design, $\bar{V}_{t}^{n}\left(S_{t}^{n, x}\right)$ is the scalar product of the values of the basis functions for post-decision state $S_{t}^{n, x}$ and the weights. Thus, the variance of $\bar{V}_{t}^{n}\left(S_{t}^{n, x}\right)$ is dependent on the weights $\theta_{t}^{n}$ and the basis functions $\phi_{t}$. We define $C_{t}^{n}$ as the $\left|\mathcal{B}_{t}\right|$ by $\left|\mathcal{B}_{t}\right|$ covariance matrix at iteration $n$ and stage $t$. The uncertainty knowledge $K_{t}^{n}$ of the approximated downstream rewards is defined as the tuple shown in (4.14).

$$
K_{t}^{n}=\left(\bar{V}_{t}^{n}, C_{t}^{n}\right)=\left(\phi_{t}, \theta_{t}^{n}, C_{t}^{n}\right)
$$

The value of exploration of a decision should be larger for those decisions that lead us to choosing a better decision in future iterations, given that we are at the same state in the same stage, than for the decision that we currently think is the best. Furthermore, the value of exploration should decrease as we explore though the iterations since the uncertainty of the estimated downstream rewards is also reduced with increasing number of observations. Eventually, when we are confident of discriminating between optimal and non-optimal decisions, only exploitation decisions should be made in order to improve the approximation $\bar{V}_{t}^{n}\left(S_{t}^{x, n}\right)$. To achieve this, VPI (as applied by Ryzhov et al. [85] in the context of infinite horizon ADP) builds upon the notion of the value of information in reinforcement learning systems [23] and defines $v_{t}^{E, n}$ using the elements of $K_{t}^{n}$ as shown in (4.15). In (4.15), the function $f$ quantifies 
the knowledge gain of an exploration decision based on the initial belief distribution, and is defined using $f(z)=z \Phi(z)+\varphi(z)$ where $\Phi$ is the cumulative distribution function and $\varphi$ the probability density function of a standard Gaussian distribution. Furthermore, $\sigma_{t}^{2, n}\left(S_{t}^{x, n}\right)$ represents the prior variance of $\bar{V}_{t}^{n}\left(S_{t}^{n, x}\right)$ and is computed as shown in $(4.15 \mathrm{c})$. For the comprehensive description and derivation of (4.15), we refer the reader to Ryzhov et al. [85].

$$
\begin{gathered}
v_{t}^{E, n}\left(K_{t}^{n}, S_{t}^{n}, x_{t}^{n}\right)=\sqrt{\sigma_{t}^{2, n}\left(S_{t}^{x, n}\right)} f\left(-\frac{\delta\left(S_{t}^{x, n}\right)}{\sqrt{\sigma_{t}^{2, n}\left(S_{t}^{x, n}\right)}}\right) \\
\text { s.t. } \\
\delta\left(S_{t}^{x, n}\right)=\left|\bar{V}_{t}^{x, n}\left(S_{t}^{x, n}\right)-\max _{y_{t}^{n} \in \mathcal{X}_{t} \mid y_{t}^{n} \neq x_{t}^{n}} \bar{V}_{t}^{x, n}\left(S_{t}^{y, n}\right)\right| \\
\sigma_{t}^{2, n}\left(S_{t}^{x, n}\right)=\phi\left(S_{t}^{x, n}\right)^{T} C_{t}^{n} \phi\left(S_{t}^{x, n}\right)
\end{gathered}
$$

In (4.15), we observe that the larger the uncertainty $\sigma_{t}^{2, n}\left(S_{t}^{x, n}\right)$ about the impact of decision $x_{t}^{n}$, the larger the value of exploration is. Besides the uncertainty, the difference between the approximated downstream rewards of decision $x_{t}^{n}$ (given by $\bar{V}_{t}^{x, n}\left(S_{t}^{x, n}\right)$ ) and the best decision of the remaining feasible decisions $y_{t}^{n} \in \mathcal{X}_{t} \mid y_{t}^{n} \neq x_{t}^{n}$ (given by $\bar{V}_{t}^{x, n}\left(S_{t}^{y, n}\right)$ ) is considered. The larger this difference is, the lower the value of exploration $v_{t}^{E, n}$ corresponding with decision $x_{t}^{n}$ becomes. Thus, the definition of $v_{t}^{E, n}\left(K_{t}^{n}, S_{t}^{n}, x_{t}^{n}\right)$ in (4.15) has the desired characteristic of reducing the risk of deteriorating our approximation through exploration decisions. To implement it, Line 5 in Algorithm 2 must be exchanged with (4.13). In addition, the covariance matrix $C_{t}^{n}$ must be initialized for all stages $t \in \mathcal{T}$ before Line 2 in Algorithm 2. Since it is difficult to define an initial distribution of belief about the true value of the approximation, the covariance matrix is usually initialized with a large number $\chi^{\mathrm{C}}$ on the diagonal and with zero on its other entries [84]. This initialization resembles the case of no prior knowledge about the relation between between the weights of the basis functions.

The approximation and the belief about its distribution must be updated after each iteration. To update the approximated downstream rewards (i.e., update the weights of the basis functions), we use (4.16) where $\hat{v}_{t}^{n}$ is the value of the exploration decision in (4.13), as calculated in Line 7 in Algorithm 2, and $\sigma^{2, \mathrm{E}}$ is a noise term due to the measurement error. This updating procedure is identical to the analogous recursive least squares method [85], and considers the difference between approximated and observed downstream rewards as well as the current uncertainty knowledge through the covariance matrix. Naturally, besides using the observed rewards, the observed basis functions can be used to update the covariance matrix. Remind that, with increasing number of observations of a post-decision state $S_{t}^{x, n}$ (i.e., observed basis functions), the uncertainty about the approximated downstream rewards of that post-decision state decreases. We update the covariance matrix as shown in (4.17), again using the noise term $\sigma^{2, \mathrm{E}}$. To implement these 
updating methods, we replace Line 12 in Algorithm 2 with (4.16) and (4.17).

$$
\begin{gathered}
\theta_{t}^{n}=\theta_{t}^{n-1}-\frac{\left(\theta_{t}^{n-1}\right)^{T} \phi\left(S_{t}^{x, n}\right)-\sum_{t}^{T^{\max }-1} \hat{v}_{t}^{n}}{\sigma^{2, \mathrm{E}}+\sigma_{t}^{2, n-1}\left(S_{t}^{x, n}\right)} C_{t}^{n} \phi\left(S_{t}^{x, n}\right) \\
C_{t}^{n}=C_{t}^{n-1}-\frac{C_{t}^{n-1} \phi\left(S_{t}^{x, n}\right) \phi\left(S_{t}^{x, n}\right)^{T} C_{t}^{n-1}}{\sigma^{2, \mathrm{E}}+\sigma_{t}^{2, n}\left(S_{t}^{x, n-1}\right)}
\end{gathered}
$$

In the updating procedure explained above, we use the downstream rewards from the exploration decisions. This differs from Ryzhov et al. [85], where the approximated downstream rewards of the exploitation decision are being used instead of the exploration decision. The use of one decision, and its value, to update the approximation and a different decision in the transition of the system is known as off-policy updating, as mentioned before. Although off-policy updating can be useful to prevent exploration decisions harming the approximation in infinite horizon problems, such as the one of Ryzhov et al. [85], it is difficult to apply it to finite horizon problems, especially in combination with backwards updating [96, 97]. To prevent exploration decisions affecting our approximation in a negative way, we propose to be slightly more conservative in three aspects of exploration: (i) the definition of "gain" in the value of exploration, i.e., $\delta\left(S_{t}^{x, n}\right)$ defined in (4.15b), (ii) the use of the value of exploration in making decisions, i.e., $x_{t}^{n *}$ defined in (4.13), and (iii) the updates resulting from exploration decisions using the noise term $\sigma^{2, \mathrm{E}}$ as shown in (4.16) and (4.17). We now elaborate on each of these aspects.

\section{The Gain in Value of Exploration}

To be more conservative with the value of exploration $v_{t}^{E, n}$, we can incorporate more aspects of the exploitation decision. The first aspect we note is the exclusion of the direct rewards $R_{t}\left(x_{t}^{n}\right)$ in the calculation of the value of exploration of decision $x_{t}^{n}$, as shown in (4.15). On the one hand, it is reasonable to use only the expected downstream rewards $\bar{V}_{t}^{x, n}\left(S_{t}^{x, n}\right)$ in (4.15) because the post-decision state corresponding to decision $x_{t}^{n}$ and its basis functions might be observed without, or with different, direct rewards at a later iteration. Remind that different states and different decisions at stage $t$ might still lead to the same post-decision state and thus the same basis function values. On the other hand, if observing basis function values would always involve direct rewards (as in the "online" use of ADP which we describe at the end of the next section), it makes sense to include them in the value of exploration. Thus, we can add $R_{t}(\cdot)$ to (4.15b), as seen in (4.18).

$$
\delta\left(S_{t}^{n}, x_{t}^{n}\right)=\left|R_{t}\left(x_{t}^{n}\right)+\bar{V}_{t}^{x, n}\left(S_{t}^{x, n}\right)-\max _{y_{t}^{n} \in \mathcal{X}_{t}^{\mathrm{R}} \mid y_{t}^{n} \neq x_{t}^{n}}\left(R_{t}\left(y_{t}^{n}\right)+\bar{V}_{t}^{x, n}\left(S_{t}^{y, n}\right)\right)\right|
$$

\section{The Exploration Decision}

Although the idea from the previous modification decreases the value of exploration for decisions with relatively low direct rewards, the exploration decision itself, as given by 
(4.13), is still solely based on the value of exploration. Another way to be conservative with the exploration decisions is to directly include, in addition to the value of exploration, some aspects of the exploitation decision (i.e., Line 5 in Algorithm 2). Naturally, there are many forms to include these aspects. We propose three forms of doing so.

First, we can include the approximated downstream reward when making a decision as proposed by Dearden, Friedman, and Andre [23] and shown in (4.19). This modification overcomes the disadvantage of making decisions that are far-from-optimal with respect to downstream rewards due to solely focusing on the value of exploration. Nevertheless, if $v_{t}^{E, n} \ll$ $\bar{V}_{t}^{x, n}$ we might explore only seldom and therefore converge to a "locally optimal" policy.

$$
x_{t}^{n, \mathrm{E} 2}=\underset{x_{t}^{n} \in \mathcal{X}_{t}^{\mathrm{R}}}{\operatorname{argmax}}\left(\bar{V}_{t}^{x, n}\left(S_{t}^{x, n}\right)+v_{t}^{E, n}\left(S_{t}^{n}, K_{t}^{n}, x_{t}^{n}\right)\right)
$$

Second, both the direct and the approximated downstream rewards can be added to the value of exploration when making a decision as proposed by Ryzhov et al. [85] and shown in (4.20). This modification ensures that towards the last iterations, when the value of exploration is approximately the same for many decisions, the exploitation decision is chosen. Exploitation in the last iterations will improve the downstream estimates if the policy learned is close to optimal. However, in this approach we need to be even more careful that the value of exploration is scaled properly, i.e., $v_{t}^{E, n} \sim\left(R_{t}\left(S_{t}^{n}, x_{t}\right)+\bar{V}_{t}^{x, n}\right)$, such that we actually do some exploration.

$$
x_{t}^{n, \mathrm{E} 3}=\underset{x_{t}^{n} \in \mathcal{X}_{t}^{\mathrm{R}}}{\operatorname{argmax}}\left(R_{t}\left(S_{t}^{n}, x_{t}\right)+\bar{V}_{t}^{x, n}\left(S_{t}^{x, n}\right)+v_{t}^{E, n}\left(S_{t}^{x, n}, K_{t}^{n}, x_{t}^{n}\right)\right)
$$

Third, we use the same rationale of the second modification but with a tighter control over the amount of exploration throughout the iterations. To achieve that in early iterations decisions are made according to traditional VPI exploration, i.e., (4.13), and in later iterations follows pure exploitation, i.e., Line 5 in Algorithm 2, we introduce a weight $\alpha^{n} \in[0,1]$, as shown in (4.21). This iteration-dependent weight is close to one in early iterations and close to zero in later ones.

$$
x_{t}^{n, \mathrm{E} 4}=\underset{x_{t}^{n} \in \mathcal{X}_{t}^{\mathrm{R}}}{\operatorname{argmax}}\left(\left(1-\alpha^{n}\right)\left(R_{t}\left(S_{t}^{n}, x_{t}\right)+\bar{V}_{t}^{x, n}\left(S_{t}^{x, n}\right)\right)+\alpha^{n} v_{t}^{E, n}\left(S_{t}^{x, n}, K_{t}^{n}, x_{t}^{n}\right)\right)
$$

The idea of exploring using the traditional VPI exploration decision in (4.13) is suitable as long as we are able to improve our approximation and it does not "cost" anything. However, some of the applications for which the idea was introduced have costs associated with exploration. This can happen, for instance, if the algorithm uses real-life observations of the exogenous information, or if the simulation of the exogenous information is so expensive that there is a limit on the number of iterations in ADP. This is the so-called "online" use of ADP [85], and for this case, the proposed modification in (4.20) seems reasonable. Although in our problem we use the "offline" version of ADP, which means we first learn the approximation without making real costs and then use the approximation to make decisions in real-life, we still suffer 
from exploration due to our finite horizon setting. Since we use backwards updating, exploration decisions at the final stages will affect the updates of the approximation at early stages. These effect would repeat over the iterations and hence the approximation that would be used after the ADP algorithm is finished would also suffer. This issue brings us to our last proposed modification: to be more conservative with updates resulting from exploration decisions.

\section{The Update of the Approximation}

The last modification we propose deals with the updates resulting from exploration decisions. Specifically, we propose adjusting the noise term $\sigma^{2, \mathrm{E}}$ in the updating equations (4.16) and (4.17). The general idea is that the higher this noise term is, the less the observed error (i.e., difference between the approximated downstream rewards and the observed ones) affects the approximations, since the difference can be partly attributed to "noise". Noise, in our context, has two causes: (i) fluctuations in the downstream rewards due to realizations of the random demand, and (ii) changes in the the policy (i.e., decisions made) due to the changing approximation $\bar{V}_{t}^{n}\left(S_{t}^{x, n}\right)$. Typically, this noise term is assumed known and constant across all stages in an infinite horizon problem. In our problem, however, this would mean that $\sigma^{2, \mathrm{E}}=$ $\eta^{\mathrm{E}} \forall t \in \mathcal{T}$, where $\eta^{\mathrm{E}}$ is the problem specific noise. This is not desirable as we explain below.

Suppose that we are at the same state and stage at iterations $n$ and $n+1$ in the ADP algorithm. Although in both situations the feasible decision space is the same, decision $x_{t}^{n+1, *}$ might differ from decision $x_{t}^{n, *}$ because, from iteration $n$ to iteration $n+1$, either the approximation $\bar{V}_{t}^{n}\left(S_{t}^{x, n}\right)$ changed or the uncertainty knowledge $K_{t}^{n}$ used to make exploration decisions changed. Since at earlier iterations changes in both the approximation and the uncertainty knowledge can be large due to their initialization, changes in the aforementioned decisions can be large. Furthermore, in a finite horizon problem with backward updates, changes in decisions at later stages would accumulate to earlier stages of the horizon, meaning that at early stages noise would be larger than at late stages. To account for this decreasing nature of noise across the stages in the horizon, or across the iterations in our ADP algorithm, we propose three forms of modifying the noise term $\sigma^{2, \mathrm{E}}$.

First, we can let the noise term $\sigma_{t}^{2, \mathrm{E}}$ depend on the stage $t$ as a linearly decreasing function of the constant noise term $\eta^{\mathrm{E}}$, as shown in (4.22). The noise term $\eta^{\mathrm{E}}$ must, nevertheless, be tuned for the problem.

$$
\sigma_{t}^{2, \mathrm{E} 2}=\frac{T^{\max }-t}{T^{\max }} \eta^{\mathrm{E}}
$$

Second, to deal with the noise due to changes in policy across the iterations, as well as stages, we can let the noise term depend on the uncertainty $\sigma_{t}^{2, n}\left(S_{t}^{x, n}\right)$ about the impact of a decision, as shown in (4.23). The logic behind this modification is that, if we choose a decision that leads us to a highly uncertain post-decision state (i.e., high variance of the approximated downstream rewards of that post-decision state), then the resulting observation will have a lesser impact on our update. In VPI, the decision to visit a highly uncertain post-decision state is likely to be an exploration decision, and these decisions 
we typically want to contribute less since they can be far from optimal.

$$
\sigma_{t}^{2, \mathrm{E} 3}\left(S_{t}^{x, n}\right)=\sigma_{t}^{2, n}\left(S_{t}^{x, n}\right)
$$

Third, we can combine the two previous ideas, as shown in (4.24). This is the most conservative of our proposals to modify the update of the approximation.

$$
\sigma_{t, n}^{2, \mathrm{E} 4}\left(S_{t}^{x, n}\right)=\frac{T^{\max }-t}{T^{\max }} \eta^{\mathrm{E}}+\sigma_{t}^{2, n}\left(S_{t}^{x, n}\right)
$$

To recap, we proposed several modifications in three aspects of the exploration decisions to decrease the risk of negatively affecting our approximation. Overall, we have the following options to apply Bayesian exploration to our problem. First, we have two options to quantify the value of exploration: (4.15) and (4.18). Second, we have four options to make an exploration decision: (4.13), (4.19), (4.20), and (4.21). Third, we have four options to define the noise in the updating: constant, stage-dependent (4.22), iteration-dependent (4.23), and stage and iteration dependent (4.24). In the following section, we investigate which of the $2 \times 4 \times 4=32$ combinations of modifications work best, and study the performance of ADP with Bayesian exploration compared to traditional ADP and other heuristics.

\subsection{Numerical Experiments}

In this section, we study the performance of our ADP designs through a series of numerical experiments. Specifically, we explore (i) the relation between the input parameters of our ADP designs and their performance in terms of learned and realized rewards, and (ii) the performance of using the learned policy from our ADP algorithms compared to a benchmark heuristic. In Section 4.6.1, we present our experimental design and setup. In Sections 4.6.2 and 4.6.3, we present and analyze the results of the two parts of our study. In Section 4.7, we finalize with a discussion on advantages and limitations of our ADP algorithms for scheduling freight in long-haul multi-transfer transport.

\subsubsection{Experimental Design}

To test our ADP algorithms, we use three transport network configurations and a planning horizon of $T^{\max }=50$ days. These three networks, and their settings, are based on the multi-modal consolidation network typologies suggested by [54], [116], and [13]. Each network increases in amount of consolidation opportunities (i.e., more services or terminals) compared to the previous one, as shown in Figures 4.2 to 4.4. In these figures, the axes denote the distance (in $\mathrm{km}$ ) between the locations in the network, $Q$ the capacity of each service in number of freights, and $L^{A}$ the duration of each service in days. The duration of truck connections is one day. Remind that we assume an unlimited number of trucks, as motivated in Section 4.4.1. We consider transfer time not to be restrictive in any terminal in the network, i.e., $L_{i, t}^{N}=0$, for all terminals $i$. 
All networks span an area of 1000x500 km, and have the same location for origins and destinations. The distances between the origins and the destinations range from 800 to $1044 \mathrm{~km}$, and the distances between an terminals close to the origin and terminals close to the destinations range from 500 to $854 \mathrm{~km}$, to resemble distances that make consolidation for the long-haul desirable in Europe according to Woxenius [116]. We use a cost structure comparable to the one proposed by [8] and [47] to represent internal and external costs of multi-modal and road freight transport networks, and especially, to incorporate the differences in costs due to economies of scale of various transport modes. For each day $t$ in the horizon, the setup cost $B_{i, j, t}$ ranges between 169 and 425 for barges and trains and the variable cost $C_{i, j, d, t}$ ranges between 37 and 868 for barges, trains, and trucks. Further details of this cost structure, its cost-saving opportunities due to consolidation, and its decision challenges are given in Appendix 4-B. For each day, the revenue $A_{i, j, d, t}$ is 868 for each freight picked up at its origin, independent of its origin or destination, and 0 otherwise. This entails that the entire revenue is received at the beginning of transport (i.e., first mile), and afterwards only costs are incurred for each freight.

The number of freights that arrives at each origin, and their destination, varies according to the probability distributions shown in Appendix 4-C. The time-window, upon arrival, is fixed to $r=0$ and $k=6$ for all freights (i.e., $p_{0, i, t}^{\mathrm{R}}=1$ and $p_{6, i, t}^{\mathrm{K}}=1$ for all origins $i$ ). This means that freights can immediately be transported, or postponed at most 2 days for a long-haul multi-modal service to be feasible. Remind that, in synchromodality, there is no restriction on the transport mode or number of transfers to use for a freight.

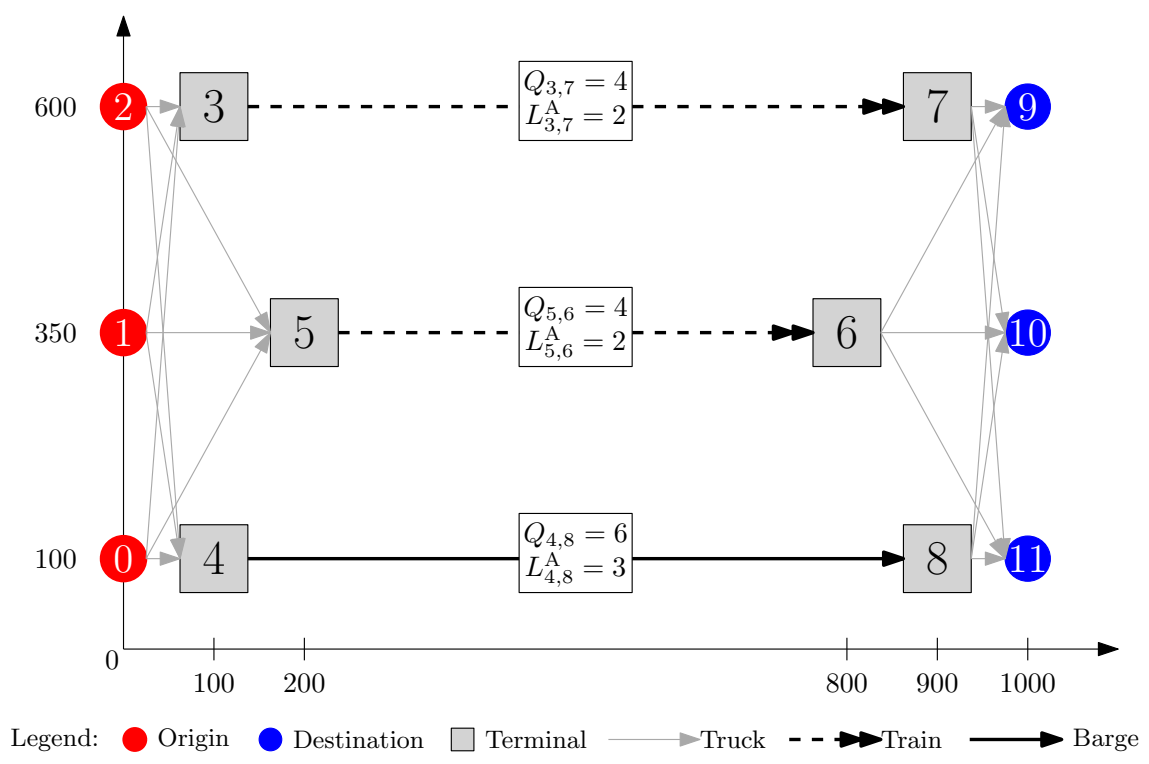

Figure 4.2: Network 1: point-to-point topology 


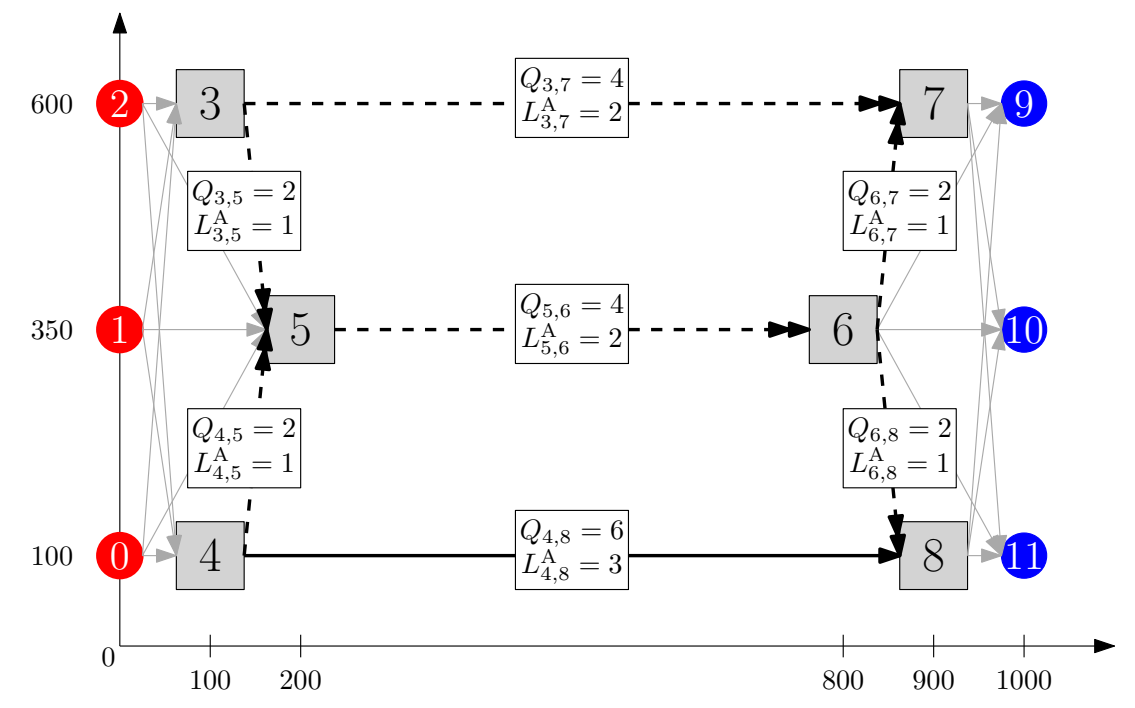

Legend: Origin Destination $\square$ Terminal $\longrightarrow$ Truck $-\rightarrow$ Train

Figure 4.3: Network 2: collection-distribution topology

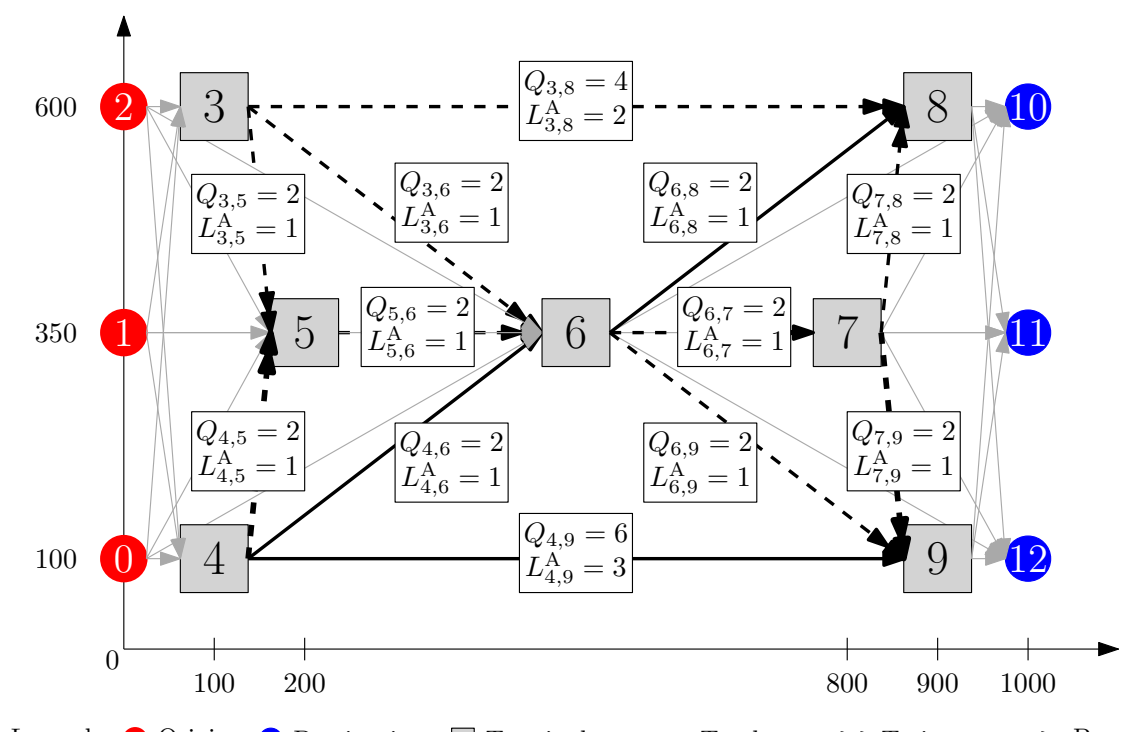

Legend: Origin Destination $\square$ Terminal $\longrightarrow$ Truck $-\rightarrow$ Train $\longrightarrow$ Barge

Figure 4.4: Network 3: hub-and-spoke topology 
The initial state $S_{0}$ for each network contains six freights, one freight of each of the following characteristics: $F_{0,11(12), 0,6,0}, F_{1,10(11), 0,6,0}, F_{2,10(11), 0,6,0}, F_{3,9(10), 0,4,0}, F_{4,11(12), 0,5,0}$, and $F_{5,11(12), 0,1,0}$ (note that destinations in Network 3 are displayed between parenthesis). This initial state contains "average" freights on the origins plus a few freights in the network. Our choice of 50 days in the planning horizon ensures that the effect of the initial state in the rewards for the entire horizon is not so large by allowing enough variability (in the arriving freights) to be present in the network. Naturally, the rewards for the horizon depend not only on the variability of the arriving freights but also on the decisions being made. For instance, postponing the transport of freights will also postpone the moment that costs are incurred. In the end of the horizon, such decisions will be deemed good if there is no end-effect. For this reason, we include an end-effect (i.e., costs after day 50) by estimating the costs to send all freights remaining in the network using the benchmark heuristic, which is presented later on in Section 4.6.3.

To evaluate the performance of our ADP designs, we carry out two types of experiments: tuning and benchmark experiments. In the tuning experiments, we test several input parameters, such as the exploration probability $\epsilon$ in our ADP design with epsilon-greedy exploration and the noise term $\eta^{\mathrm{E}}$ in our ADP design with Bayesian exploration. Furthermore, we test the 32 possible VPI modifications. Our goal in these experiments is to provide insights into the relation of these parameters and their performance. We describe these experiments in detail and present their results in Section 4.6.2. In the benchmark experiments, we compare the performance of the best parameters of our two ADP designs resulting from the tuning experiments to a benchmark heuristic. Furthermore, we compare our best ADP design with the benchmark heuristic using various time-window settings. Our goal is to study the relation between time-window characteristics and the gains or losses of using ADP over using the benchmark heuristic. We describe these experiments and present their results in Section 4.6.3.

\subsubsection{Tuning Experiments}

In our tuning experiments, we study the two ADP designs from Section 4.5. We test the various modifications of our ADP design with Bayesian exploration and compare them to our ADP design with epsilon-greedy exploration. To make the comparison fair, we test several input values for the tunable parameters of each of the two designs, under the same conditions. Before presenting the results, we first describe the conditions under which we test our designs and the input values we use for the tunable parameters.

As described in Section 4.5.2, we are interested in the offline use of ADP, which is to first learn the approximation of downstream rewards within a simulated environment, and then use the approximation to make real-life decisions. For this reason, we measure performance in two ways: (i) learned approximation of downstream rewards after running the ADP algorithm, which we call learned rewards, and (ii) realized rewards of using the approximation for making decisions in a simulation, which we call realized rewards. The two of them are related to the MDP model's objective function in (4.6), the first relates to the optimal expected discounted rewards, and the second one relates to the policy that obtains these rewards. Although related, these two performance measurements are not necessarily the same. The basis function weights that our ADP algorithms learn can be 
far from the optimal rewards, but the resulting policy can be close to the optimal policy.

For each ADP design, we use $N=50$ iterations and common random numbers, i.e., freights that arrive during each day of each iteration are the same across tested designs. Common random numbers are used to rule out differences in arrival processes of freights through the iterations of the ADP algorithms as the source of differences in the learned and realized rewards among them. To test the resulting policy of each ADP design, we use 50 simulation runs of the planning horizon and, again, common random numbers which are different from the learning phase. Remind that the resulting policy, or function that maps each state to a decision, is given by the weights of the basis functions $\left[\theta_{t}^{n}\right]_{\forall t \in \mathcal{T}}$ and the restricted policies RP 1 (i.e., $\mathcal{X}_{t}^{\mathrm{RP} 1}$ ) and RP 2 (i.e., $\mathcal{X}_{t}^{\mathrm{RP} 2}$ ) defined in (4.8) and (4.9), respectively. These restricted policies significantly reduce the number of feasible decisions to evaluate. For example (see Appendix 4-B), in a given state that has $2.6 \times 10^{8}$ feasible decisions, RP 1 reduces the number of decisions to evaluate to $1.9 \times 10^{4}$ and $\mathrm{RP} 2$ reduces them to $5.8 \times 10^{4}$. These reductions, however, limit the performance that ADP can attain in learned and simulated rewards.

For the ADP design with $\epsilon$-greedy exploration, we test three values of $\lambda=\{0.01,0.1,1\}$, which control the emphasis on recent observations during the updates in (4.12). We test four values of the probability of exploration $\epsilon=\{0,0.3,0.6,0.9\}$. Remind that $\epsilon=0$ means only exploitation decisions, while $\epsilon=1$ means only exploration decisions. Furthermore, we test two ways of initializing the weights of the basis functions: (i) $\theta_{b, t}^{0}=0$ for all characteristics $b \in \mathcal{B}$ and days $t \in \mathcal{T}$, and (ii) $\theta_{|\mathcal{B}|, t}^{0}=\beta\left(T^{\max }-t\right) / T^{\max }$ for the constant basis function and $\theta_{b, t}^{0}=0$ for all other characteristics $b \in \mathcal{B}|b \neq| \mathcal{B} \mid$ and days $t \in \mathcal{T}$. The first initialization represents a case where we have no knowledge about the weights of the approximated rewards, and the second one represents a case where we have an estimate of the magnitude of the total downstream rewards $\beta$. In our case, $\beta$ is defined as the rewards attained by the benchmark heuristic: $\beta=38,036$ for Network $1, \beta=33,445$ for Network 2 , and $\beta=33,889$ for Network 3 .

For the ADP design with Bayesian exploration, we initialize the basis function weights using the second option described before. We test four values of the noise term $\eta^{\mathrm{E}}=$ $\left\{10^{2}, 10^{4}, 10^{6}, 10^{8}\right\}$. For the initial covariance matrix, we test four values for the diagonal $\chi^{\mathrm{C}}=\left\{10,10^{2}, 10^{3}, 10^{4}\right\}$. We base our settings on Ryzhov et al. [85], who recommend that $\eta^{\mathrm{E}}>\chi^{\mathrm{C}}$ and that their ratio is of the order 10 or $10^{2}$. For the weight $\alpha^{n}$ in modification (4.21), we test $\alpha^{n}=\{1 / n, 10 /(n+9), 100 /(n+99)\}$. We test all the parameters described above for each of the 32 combinations of VPI modifications that we propose in Section 4.5.2.

Testing all tunable parameters and modifications of our two ADP designs for the three experimental networks results in more than 3500 experiments. Each experiment provides the learned and the realized rewards of ADP. Before discussing the details of the relation between $\mathrm{ADP}$ performance and the tunable parameters/modifications, we limit ourselves to present the results of the best parameters and modifications in Table 4.1, which consists of (i) the result for the tuned value of $\epsilon, \lambda$, and $\beta$ for the ADP design with $\epsilon$-greedy exploration, and (ii) the tuned value of $\eta^{\mathrm{E}}, \chi^{\mathrm{C}}$, and $\alpha^{n}$, and the best combination of modifications in Section 4.5.2 for the ADP design with Bayesian exploration. We use the following acronyms within the tables and figures of this section: Restricted Policy (RP), Basis Functions (BF), and Value of Perfect Information (VPI). 
Table 4.1: Maximum realized reward (i.e., tunned settings) and their corresponding learned reward for various $A D P$ designs

\begin{tabular}{|c|c|c|c|c|c|c|c|}
\hline \multirow{3}{*}{\multicolumn{2}{|c|}{ ADP Design }} & \multicolumn{2}{|c|}{ Network 1} & \multicolumn{2}{|c|}{ Network 2} & \multicolumn{2}{|c|}{ Network 3} \\
\hline & & Realized & Learned & Realized & Learned & Realized & Learned \\
\hline & & Rewards & Rewards & Rewards & Rewards & Rewards & Rewards \\
\hline \multirow{3}{*}{$\mathrm{RP} 1$} & $\mathrm{BF}$ & $-7,994$ & 38,219 & $-11,247$ & 33,720 & $-16,548$ & $-17,928$ \\
\hline & $\mathrm{BF}+\epsilon$-greedy & $-4,628$ & $-6,984$ & $-11,485$ & 33,228 & $-18,172$ & $-18,507$ \\
\hline & $\mathrm{BF}+\mathrm{VPI}$ & 34,044 & 36,571 & 34,284 & 29,493 & 34,898 & 23,285 \\
\hline \multirow{3}{*}{$\mathrm{RP} 2$} & $\mathrm{BF}$ & $-4,912$ & $-3,803$ & $-11,734$ & 34,060 & $-11,949$ & 34,495 \\
\hline & $\mathrm{BF}+\epsilon$-greedy & 880 & 37,386 & $-11,450$ & $-12,091$ & $-11,949$ & 33,356 \\
\hline & $\mathrm{BF}+\mathrm{VPI}$ & 40,439 & 35,407 & 40,195 & 31,107 & 38,314 & 30,791 \\
\hline
\end{tabular}

In Table 4.1, we observe the maximum realized rewards over all settings of the tunable parameters and VPI modifications, and their corresponding learned reward, for each ADP design. Three observations stand out. First, using VPI instead of the traditional $\epsilon$-greedy strategy for exploration significantly improves the realized rewards. In fact, the policy resulting from the $\epsilon$-greedy strategy, although better in most cases than the exploitation only (i.e., $\mathrm{BF}$ in the table), ends up in costs (i.e., negative rewards). Second, the difference in realized rewards between the two restricted policies varies per network and per design. Consider for instance Network 2, where the difference between the $\mathrm{BF}$ and $\mathrm{BF}+\epsilon$-greedy design across RP 1 and RP 2 is much smaller than the one from BF + VPI. Third, the accuracy of the approximation (i.e., difference between learned and realized rewards) varies per network and RP option for the traditional designs, but is more consistent for the BF+VPI design. BF+VPI underestimates the rewards by at most 11,000 whereas the traditional designs overestimate the rewards by at most 45,000 . Although our focus is not on the learned rewards, these observations lead to discussion points, which we address in Section 4.7. We now focus on the relation between the tunable parameters/modifications of each ADP design and the realized rewards.

In the ADP design with basis functions and $\epsilon$-greedy exploration, the tuned settings of most networks and RP options (all but one) ended up in negative rewards as seen in Table 4.1. All settings different from the tuned ones performed the same or worse. In practice, this means that the policy resulting from this ADP design would never be implemented. Therefore, we limit ourselves to two general observations only. First, there is no significant difference with respect to the way weights are initialized in each network and RP option. Second, there is at least one value of $\epsilon>0$ that performs better than $\epsilon=0$ for Network 1 and Network 2, but not in Network 3. Since this last observation hints that exploring pays off, but is not sufficient to fully escape the local-optima of exploitation decisions, we now focus on the analysis of our ADP + VPI design, which significantly outperforms the traditional design.

Before analyzing the VPI modifications, we analyze the tuning of the noise and Bayesianbelief related parameters. In Figure 4.5, we provide a comparison of different ratios $\eta^{\mathrm{E}} / \chi^{\mathrm{C}}$. In line with Ryzhov et al. [85], we observe that with ratios of $10^{2}$ through $10^{4}$, VPI works best on average over all modifications for both RPs. From this figure, we can gather two important insights for the ratio $\eta^{\mathrm{E}} / \chi^{\mathrm{C}}$. First, realized rewards and accuracy seem to 

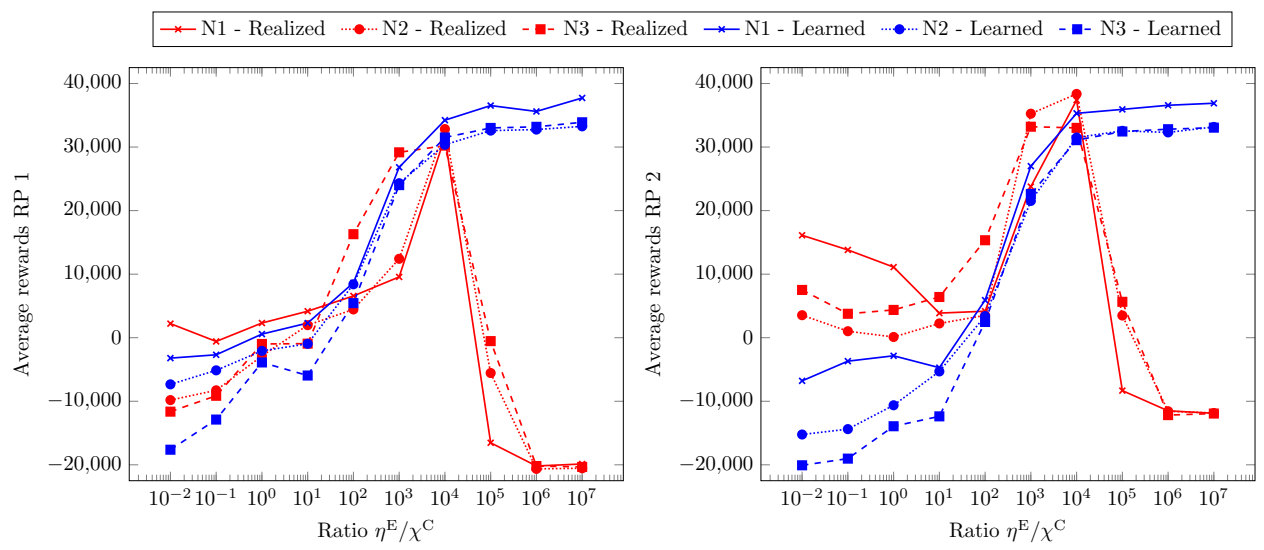

Figure 4.5: Comparison of average rewards (over all modifications) under different ratios $\eta^{E} / \chi^{C}$

improve with an increasing ratio until the best one (in our case $10^{4}$ ). At the ratio with the best realized rewards we also find the smallest difference with the learned rewards. Second, at ratios larger than the "best-tuned" one, realized rewards rapidly decrease to the point of becoming costs rather than rewards, even though the learned rewards remain the same.
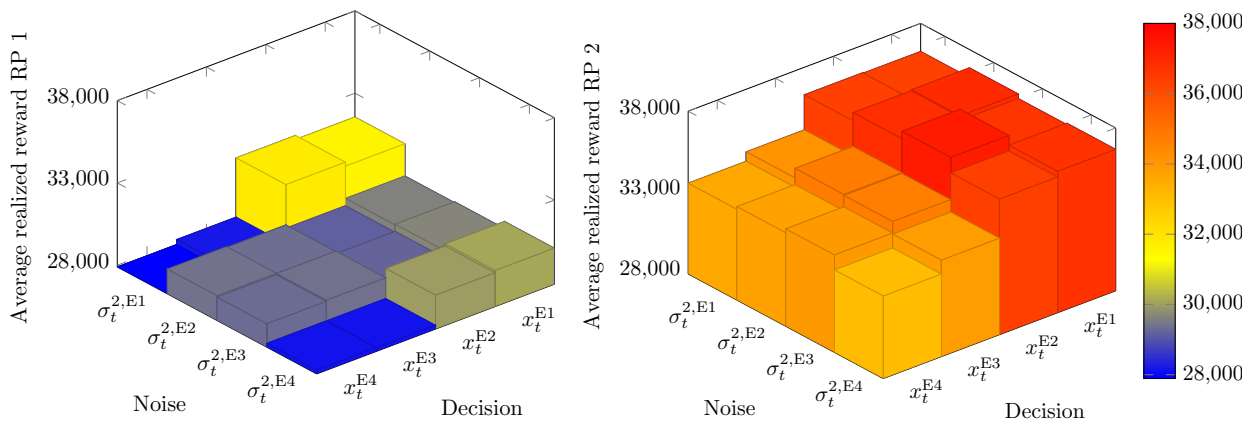

Figure 4.6: Comparison of average rewards (over all networks) for our proposed VPI modifications

At last, we analyze the performance of our VPI modifications. In Figure 4.6, we show the average realized rewards (over all networks) as a function of the decision and noise modifications explained in Sections 4.5.2 and 4.5.2, respectively. We exclude from our analysis our second proposed way of defining the value of exploration using the direct rewards since this performed significantly worse than the original definition of the value of exploration, for all decision and noise modifications. In Figure 4.6, the superscript E1 represents the original noise and decision definition in VPI, meaning that the upper corner corresponds 
to the original VPI design. We observe a significant difference between the average realized rewards for all modifications in RP 1 compared to RP 2, with all modifications in RP 2 performing better than the best modification in RP 1 . This is to be expected since RP 2 has more decision freedom than RP 1 . We also observe that, in RP 2, the modifications of how to make a decision have a larger impact than the modifications to the noise used when updating the approximation. Overall, including the downstream rewards in addition to the value of exploration when making decisions (i.e., $x_{t}^{\mathrm{E} 2}$ ) and letting the noise depend only on the post-decision state of the given stage and iteration (i.e., $\sigma_{t}^{\mathrm{E} 3}$ ) are the best modifications tested for our problem. It seems that including the downstream rewards instead of the direct rewards helps the algorithm avoid the aforementioned greedy behavior and related worse performance. It also seems that considering the uncertainty of the post-decision state instead of a constant noise term when updating pays off. Using modifications $x_{t}^{\mathrm{E} 2}$ and $\sigma_{t}^{\mathrm{E} 3}$ for our BF+VPI design, we continue to our benchmark experiments.

\subsubsection{Benchmark Experiments}

In our benchmark experiments, we compare the realized rewards of our best ADP design (i.e., $\mathrm{BF}+\mathrm{VPI}$, with RP 2 and modifications $x_{t}^{\mathrm{E} 2}$ and $\sigma_{t}^{\mathrm{E} 3}$ ) against a Benchmark Heuristic (BH). The objective is to compare the use of the learned ADP policy against a simpler but effective scheduling heuristic. The BH aims to use the multi-modal services efficiently, i.e., consolidating as many freights as possible in a service once the setup costs for using that service can be covered. The BH consists of fours steps: (i) define the shortest and second shortest path for each freight to its final destination, considering only variable costs for services between terminals, (ii) calculate the savings between the shortest and second shortest path and define these as savings of the first multi-modal service used in the shortest path, (iii) sort all freights in non-decreasing time-window length, i.e., closest due-day first, and (iv) for each freight in the sorted list, check whether the savings of the first multi-modal service of its shortest path are larger than the setup cost for using this service; if so, use this service for the freight, if not, postpone the transport of the freight. The pseudo-code for this heuristic can be found in Appendix 4-E. Note that the $\mathrm{BH}$ is not subject to the restrictions imposed by RP 2, as ADP is.

Using the three networks from the previous section plus additional time-window distributions, we set up our experiments as follows. For each network, we replicate ten times the process of learning the ADP weights and simulating the use of the resulting policy. The use of the $\mathrm{BH}$ is also replicated ten times, using common random numbers with the corresponding ADP part, such that differences arise due to the scheduling differences and not the arriving freights. Identical to the tuning experiments of the previous section, one replication of the ADP process consists on running the ADP algorithm for 50 iterations and simulating the entire planning horizon 50 times. For the networks with different time-window distributions, we re-tune the noise parameters, since they are dependent on the inherent uncertainty of the problem. Here we fix the best ratio $\eta^{\mathrm{E}} / \chi^{\mathrm{C}}$ found, i.e., $10^{4}$, but increase the values of $\eta^{\mathrm{E}}$ and $\chi^{\mathrm{C}}$.

Table 4.2 shows the average realized rewards, over the ten replications, using the same networks from the tuning experiments of the previous section. Remind that these networks have uncertainty in the amount of freight that arrives and their destination, but not in their timewindow. We observe that, for Network 1, ADP performs slightly better than the benchmark 
Table 4.2: Average realized rewards (over the replications) of the $B H$ and $A D P$

\begin{tabular}{|c|c|c|c|c|c|c|}
\hline \multirow{2}{*}{ Scheduling policy } & \multicolumn{2}{|c|}{ Network 1} & \multicolumn{2}{|c|}{ Network 2} & \multicolumn{2}{|c|}{ Network 3} \\
\hline & Average & Gain & Average & Gain & Average & Gain \\
\hline $\mathrm{BH}$ & $37,347.36$ & - & $33,066.79$ & - & $32,963.79$ & - \\
\hline ADP with RP 2 & $37,502.84$ & $0 \%$ & $36,867.96$ & $11 \%$ & $33,839.92$ & $3 \%$ \\
\hline
\end{tabular}

heuristic, but not with a significant difference. In Networks 2 and 3, however, ADP performs significantly better than the heuristic, with the difference between the two being the largest in Network 2. The differences in performance of both the BH and ADP across the networks seem to indicate two traits about scheduling freight in long-haul multi-transfer transport. First, the larger the complexity of the network is, the lower the average realized rewards are (remind that Network 1 is the simplest and 3 the most complex). Second, the gain of using ADP seems to be the largest in a more complex network (comparing Networks 1 and 2) up to a certain extent (comparing Networks 2 and 3). We come back to these traits in the following section.

In the experiments above, all freights that arrive are immediately released and have a timewindow length of six days. With this time-window length, freights can be postponed at most 2 days for a long-haul multi-modal service to be feasible. However, the length and uncertainty of the time-window of freights may affect the performance of an ADP algorithm [71]. To test this, we design three distributions for Release-Days (RD) and three distributions for TimeWindow (TW) lengths, as shown in Tables 4.6 and 4.7 in Appendix 4-C. Each distribution is categorized as short, medium, or long. Short RD means that $60 \%$ of the freights are released immediately while long RD means that $60 \%$ of the freights are released two days after arriving. Short TW means that $60 \%$ of the freights must be at their destination within 4 days after being released (i.e., cannot be postponed after released if a long-haul multi-modal service is desired) while long RD means that $60 \%$ of the freights have a time-window length of 6 days. We follow the same procedure as before, with ten replications. Absolute results are shown in Table 4.8 (see Appendix 4-D) and relative results are shown in Figure 4.7.

As can be expected, the longer the distribution of time-window length, the larger the realized rewards are for both scheduling methods in each network, independent of the release-day. This happens because both methods are able to postpone the transport of freight and anticipate on better consolidation opportunities. With respect to the performance of ADP, we observe that with short distributions of time-window lengths, the benchmark heuristic is better. This is reasonable, since there are less options for postponing freights, and hence if a wrong postponement decision is made, the freight would have to be transported with an expensive alternative. Furthermore, it is reasonable that ADP performs worse than the BH since it is subject to the restrictions of RP 2, which enforces decisions on groups of freights rather than individual freights. With respect to RD distributions, we observe that ADP performs slightly better with short release-day distributions. Finally, Figure 4.7 seems to indicate that our ADP approach is useful in Network 2 but not in Networks 1 and 3. However, the weakness in our approach can be attributed mostly to the restricted decision space (RP 2) instead of to the learning of the basis function weights. In the following, we show the effect of RP 2 on our problem. 


\section{IIS-RD 目目M-RD IDL-RD}

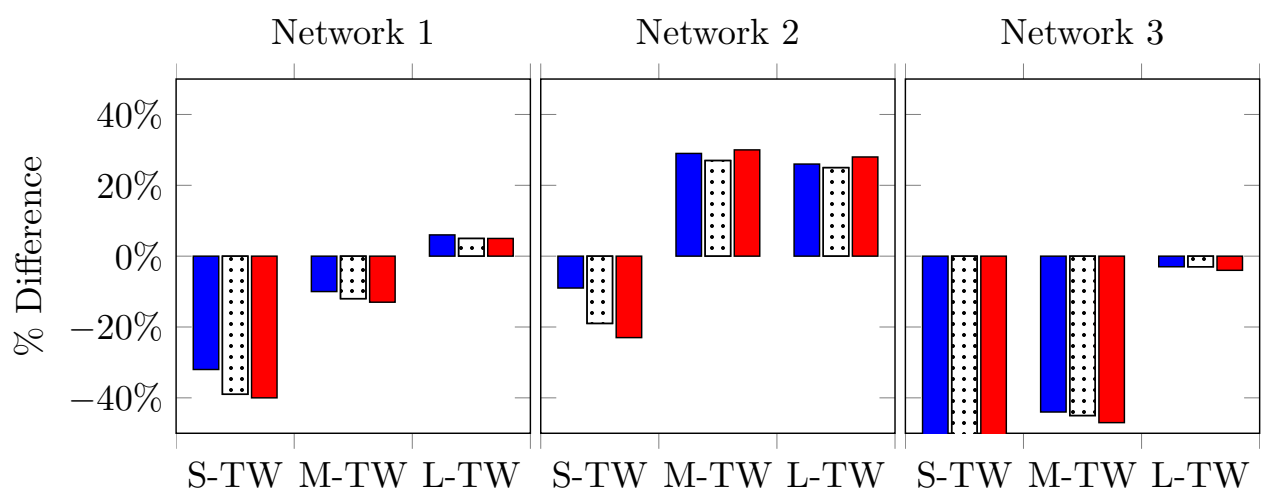

Figure 4.7: Percentage gain of ADP RP 2 over BH with respect to short (S), medium (M), and long $(L)$ release-days $(R D)$ and time-window $(T W)$ lengths.

Suppose that the problem itself requires the two grouping restrictions of freights in RP 2: (i) all released freights that go to the same destination in each multi-modal terminal must be transported together and (ii) all released freights in all origins that go to the same destination must be transported together. For ADP, these restrictions were already in place. For the $\mathrm{BH}$, these restrictions mean that instead of doing the four steps for each freight, the four steps are done for each group of freights that fulfill the conditions of RP 2 . We show the comparison between ADP and the BH with RP 2 in Table 4.3, Table 4.9 (see Appendix 4-D), and Figure 4.8. The same setup for Table 4.2, Table 4.8 (see Appendix 4-D), and Figure 4.7, is used, respectively.

Table 4.3: Average realized rewards (over the replications) considering $R P 2$

\begin{tabular}{|c|c|c|c|c|c|c|}
\hline \multirow{2}{*}{ Scheduling policy } & \multicolumn{2}{|c|}{ Network 1} & \multicolumn{2}{|c|}{ Network 2} & \multicolumn{2}{|c|}{ Network 3} \\
\hline & Average & Gain & Average & Gain & Average & Gain \\
\hline BH with RP 2 & $10,442.32$ & - & $9,911.81$ & - & $9,734.21$ & - \\
\hline ADP with RP 2 & $37,502.84$ & $259 \%$ & $36,867.96$ & $272 \%$ & $33,839.92$ & $248 \%$ \\
\hline
\end{tabular}

In Table 4.3, we observe that ADP significantly outperforms the BH with RP2 in all networks from the tuning experiments (i.e., no uncertainty in time-windows), with Network 2 resulting in the largest gain. With respect to the $u$ uncertainty in time-windows, we observe that in each RD distribution, both scheduling policies achieve larger rewards with increasing TW distribution, although the differences are significantly larger for ADP. It seems that the longer the time-window is, the more ADP can look ahead for consolidation 
of freights compared to the BH with RP 2. Furthermore, although rewards across TW distributions differ among themselves, they look similar across RD distributions for each TW distribution (e.g., Medium TW distribution for Network 2 looks similar for Short, Medium, and Long RD). This makes sense since freights can be postponed only until their time-window allows it, independent of whether it is released immediately or in two days.

In Figure 4.8, we observe that our ADP design always outperforms the $\mathrm{BH}$ except for Network 3 with short time-window distribution. Actually, it is to be expected that ADP is less useful when there are short time-windows since postponement may directly result in using the expensive alternative mode (i.e., truck). In other words, anticipatory decisions do not make sense in such a problem. We also observe that for each TW distribution, the gains of ADP are larger in the case of short RD. There are two possible reasons for this: (i) the basis functions do not include any feature about release-days, so it is difficult (impossible) to learn among post-decision freights with different release-days, (ii) when there are short release-days, rewards and costs are observed earlier, which improves the learning of the feature weights. These two reasons hint that the design of the approximation itself (i.e., the choice of basis functions) in transport problems such as ours should take into account the time when rewards and costs are realized and their constraints.

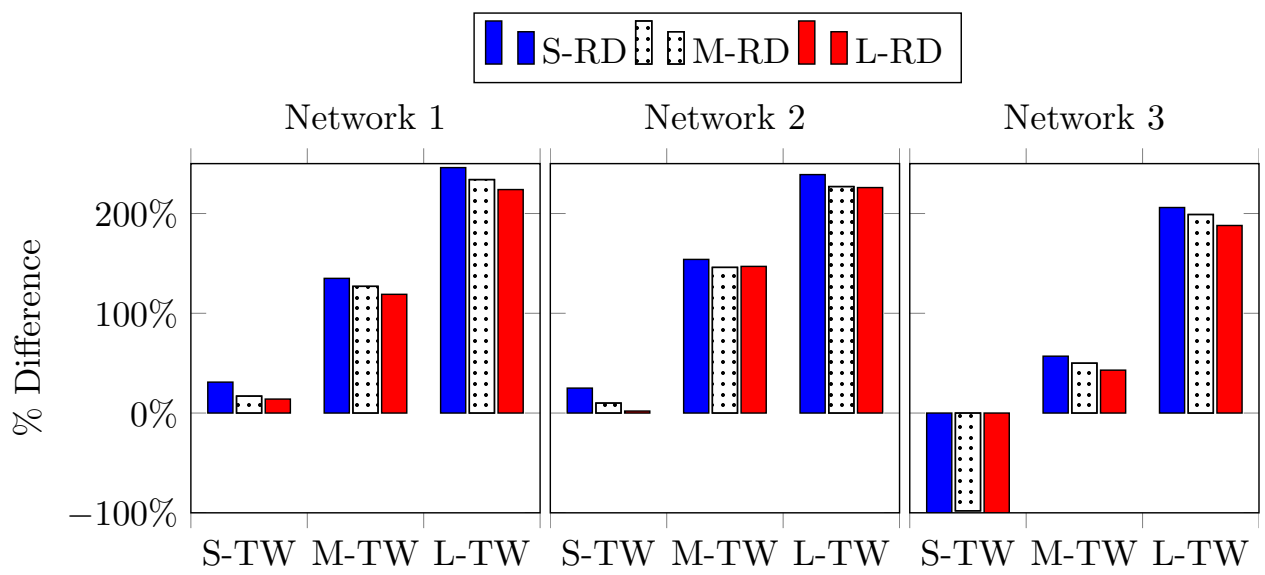

Figure 4.8: Percentage gain of $A D P R P$ 2 over $B H R P$ 2 with respect to short (S), medium $(M)$, and long $(L)$ release-days $(R D)$ and time-window $(T W)$ lengths.

In general, our experiments show that including VPI in ADP improves its performance compared to traditional designs, and that this new design can lead to substantial gains over a benchmark heuristic for various problem settings. However, they also showed that a "one-size fits all" ADP solution is hard to achieve, and some tuning is necessary. In the following section, we reflect upon our results and discuss their implications. 


\subsection{Discussion}

In our tuning experiments, we observed differences between the learned and realized rewards for most ADP designs. We observed that in the traditional ADP designs, a large learned reward did not necessarily results in a good policy. Most of the times, the learned rewards were positive while the realized rewards were negative. Although the dilemma of whether to focus on learning close-to-optimal rewards or a good policy relates to the issue of online or offline use of ADP, one can argue that both focuses are closely related and should, in theory, yield similar results. When looking for causes of the mismatch between learned and realized rewards, one can argue that problem characteristics such as the cost structure (i.e., revenue received at the beginning of transport) and restricted decision space (i.e., some post-decision states are not attainable) have a strong influence on the mismatch. Nevertheless, we observe that through the inclusion of VPI, ADP is able to tackle these issues and significantly improve the results of traditional designs, both in learned and realized rewards. More specifically, we observed that during the learning phase, combining downstream rewards with the value of exploration in VPI was the best way of making exploration decisions, and that incorporating the uncertainty of a post-decision state when updating was the best way to update the approximation. Another explanation of the initially poor performance of $\mathrm{ADP}$ could lie in the design of the VFA (i.e., basis functions), where we used simple linear basis functions. Although our primary aim was to show that, even with such a simple VFA, a better performance could be achieved by properly balancing the exploration/exploitation trade-off using Bayesian learning techniques. In Appendix 4-F, we further discuss how a more sophisticated VFA could be designed for our problem.

In our benchmark experiments, we observed that the restricted decision space is the weak point of our design. Compared to the benchmark heuristic without restrictions, ADP achieved gains in only a few problem instances. Nevertheless, if we considered that the restricted decision space is part of the problem, ADP outperformed the benchmark heuristic drastically. These improved gains provide an indication of the gains that are possible if restrictions in the decision space of ADP would be removed. However, removing restrictions in the decision space can make it more computationally complex, which in turn can make exploration in the ADP learning phase more challenging. For example, we observed that the best rewards attained by ADP were in Network 1, which has the least complex decisions of all three networks. Naturally, exploration is more difficult in settings with multi-period traveling times where multiple mode transfers are possible, such as in Networks 2 and 3, since the consequences of decisions span more than one time period. This relation between restricted decision space and computational effort needs further research. There are at least three lines worth of consideration. First, restricting the policy during early iterations of ADP and then removing the restrictions in later iterations may overall result in a better policy learned within reasonable time. Second, using a heuristic decision policy rather than restrictions might also result in a better policy with less computational burden. Third, learning a good policy for a simple network (e.g., Network 1) and then using this feasible policy as starting approximation for a more complex network (e.g., Network 2) can also reduce the need for a large number of iterations. 


\subsection{Conclusions}

In this chapter, we developed an MDP model for the anticipatory scheduling of freight in a long-haul multi-transfer transport network and a heuristic solution based on ADP. We designed various ADP algorithms using the traditional constructs of basis functions and $\epsilon$-greedy exploration, as well as methods from Bayesian exploration, specifically VPI. We described how the one-step look-ahead perspective of traditional ADP can make the algorithm flounder and end in a local-optimum, and how the ADP algorithm can escape this local-optimum and at the same time improve the solution by using the value of exploration from VPI. We proposed various modifications to VPI for infinite horizon problems to make it applicable to finite horizon ADP designs.

In a series of numerical experiments, we evaluated our ADP designs and our proposed modifications to VPI and provided insight into which modifications and tunable settings work best. We showed how VPI significantly improves the traditional $\epsilon$-greedy strategy in a finite horizon problem, as long as exploring and updating in VPI is done slightly more conservative than in the original application of VPI in infinite horizon problems. We exemplified how ADP and VPI achieve significant gains compared to a benchmark heuristic, under different demand patterns in the transport network. Finally, we reflected on the limitations of our study and possible ways to tackle these limitations. Further research about approximation architectures, the reduction of the decision space, simplification of the network during the learning phase, and robustness of VPI settings in finite horizon problems is necessary for ADP to achieve the best performance in the scheduling of freight in a long-haul multi-transfer transport network considering demand uncertainty and performance over time.

\section{Appendices}

\section{4-A Modeling Assumptions}

To simplify the formulation of the MDP model in Section 4.4, we make several assumptions and enforce certain conditions. These assumptions and conditions apply to our model, but not necessarily to our problem. In this Appendix, we briefly describe the possible modifications to the MDP model such that each assumption or condition can be bypassed.

The first condition we impose in our model is the separation between origin, multi-modal, and destination nodes. If a multi-modal terminal is also an origin or destination of freights, a duplicate node can be included in the set of origins or destinations and their related parameters can be changed accordingly. The second assumption in our model relates to the unlimited capacity for the first and third type of arcs, i.e., services beginning at an origin or ending at a destination. In other words, we assume that the pre- and end-haulage operations of our synchromodal network are not restrictive. If there is a restriction, this must be added to the decision space and, in case of penalization or loss of freight, the transition function must be changed accordingly.

One of the modeling challenges we avoid is having more than one service between two terminals. We mentioned before that duplicate multi-modal nodes can be added, and that 
services need to be modified accordingly. However, this modification is more than just altering the arcs, it involves: (i) modifying the transition function such that the same freights appear/disappear in the duplicated nodes and (ii) modifying the decision space constraints such that service capacities going to/from the duplicated nodes are respected and such that no more than the existing freights (i.e., not duplicated) can be transported.

\section{4-B Experimental Cost Structure, Consolidation Opportunities, and Decision Challenges}

To model the costs and the effects of freight consolidation (i.e., setup costs), we use the costs per $\mathrm{km}$ presented in [8] and the model with which they were calculated originally in [47]. This cost model was developed for internal and external costs of multi-modal and road freight transport networks, and the logic behind it is that costs decrease non-linearly with distance and at different rates depending on the mode. The variable cost (i.e., euro per $\mathrm{km}$ ) for truck is $5.46 d^{-0.278}$, for train is $0.58 d^{-0.26}$, and for barge is $0.46 d^{-0.26}$, where $d$ is the distance between two locations. For the fixed or setup cost (i.e., euro per service independent of the number of freight) of a service for the train is $q\left(\left(1560^{0.74}\right) / q+40\right)$ and for barge is $0.8 q\left(\left(1560^{0.74}\right) / q+40\right)$, where $q$ is the capacity of the service. We refer the reader to [47] for a thorough explanation on the cost model. In the next paragraph, we describe the consolidation opportunities and challenges of making decisions in each network.

Network 1 represents the so-called point-to-point topology [54]. Although there are no transfers in this network, there are three consolidation opportunities for each origin, namely the two train and barge services. The complexity of the decisions in this network is two-fold: (i) the restrictions imposed by the capacity of each service and (ii) the relation between the transport duration with the time-window of freights. Network 2 represents the so-called collection-distribution topology [54]. In this network, there are four additional services, and new transfers connected to the central terminals, compared to Network 1. The new consolidation opportunities bring two additional challenges to those of Network 1, the trade-off of using truck against truck-and-train to (i) bring a freight from its origin to the start of the long-haul and (ii) bring a freight from the end of the long-haul to its destination. Network 3 represents the so-called hub-and-spoke network topology [54]. In this network, there is one additional terminal, four additional services, and new transfers connected to the new terminal, compared to Network 2. The number of paths a freight can take from its origin to its destination significantly increases, and thus the complexity of the decisions increases as well. To exemplify this complexity, we compare the number of decisions in the feasible decision space of Network 3 in the following paragraph.

In Network 3, freight from any origin can be transported to any of the closest four terminals, transported to its destination, or postponed. Now, if we would have one released freight with the maximum time-window in each origin, for each destination, we would have $3 \times 3 \times(4+1+1)=54$ possible decisions for freights at the origins. Now, consider we have similar freights in Terminals 3, 4, and 6, which all have three services, and we will have 45 possible decisions for freights at those terminals, and $54 \times 45=2430$ decisions for freight at origins and Terminals 3, 4, and 6. Following a similar logic, we would end-up with $4.3 \times 10^{5}$ 
possible decisions in the network if at each terminal there would be one released freight with a large enough time-window (i.e., for services to be feasible) for each destination. If there would be two freights instead of one, we would end up with $2.6 \times 10^{8}$ possible decisions. In contrast, the same two freights with a large enough time-window for each destination, at each location, would end up in $1.9 \times 10^{4}$ in the restricted policy 1 and $5.8 \times 10^{4}$ in the restricted policy 2 defined in (4.8) and (4.9), respectively.

\section{4-C Experimental Probability Distributions}

Table 4.4: Freight probability distributions for all networks

\begin{tabular}{|c|c|c|c|c|c|}
\hline \multicolumn{2}{|c|}{ Origin 0} & \multicolumn{2}{|c|}{ Origin 1} & \multicolumn{2}{|c|}{ Origin 2} \\
\hline $\begin{array}{c}\text { Freights } \\
\quad f\end{array}$ & $\begin{array}{l}\text { Prob. } \\
p_{f, 0, t}^{\mathrm{F}}\end{array}$ & $\begin{array}{c}\text { Freights } \\
f\end{array}$ & $\begin{array}{l}\text { Prob. } \\
p_{f, 1, t}^{\mathrm{F}}\end{array}$ & $\begin{array}{c}\text { Freights } \\
f\end{array}$ & $\begin{array}{l}\text { Prob. } \\
p_{f, 2, t}^{\mathrm{F}}\end{array}$ \\
\hline 0 & 0.14 & 0 & 0.22 & 0 & 0.37 \\
\hline 1 & 0.27 & 1 & 0.33 & 1 & 0.37 \\
\hline 2 & 0.27 & 2 & 0.25 & 2 & 0.18 \\
\hline 3 & 0.18 & 3 & 0.13 & 3 & 0.06 \\
\hline 4 & 0.14 & 4 & 0.07 & 4 & 0.02 \\
\hline
\end{tabular}

Table 4.5: Destination probability distributions for all networks

\begin{tabular}{|c|c|c|c|c|c|}
\hline \multicolumn{2}{|c|}{ Origin 0} & \multicolumn{2}{|c|}{ Origin 1} & \multicolumn{2}{|c|}{ Origin 2} \\
\hline $\begin{array}{l}\text { Destination* } \\
d\end{array}$ & $\begin{array}{l}\text { Prob. } \\
p_{d, 0, t}^{\mathrm{D}}\end{array}$ & $\begin{array}{c}\text { Destination* } \\
d\end{array}$ & $\begin{array}{l}\text { Prob. } \\
p_{d, 1, t}^{\mathrm{D}}\end{array}$ & $\begin{array}{c}\text { Destination* } \\
d\end{array}$ & $\begin{array}{l}\text { Prob. } \\
p_{d, 2, t}^{\mathrm{D}}\end{array}$ \\
\hline $9(10)$ & 0.1 & $9(10)$ & 0.33 & $9(10)$ & 0.14 \\
\hline $10(11)$ & 0.1 & $10(11)$ & 0.34 & $10(11)$ & 0.29 \\
\hline $11(12)$ & 0.8 & $11(12)$ & 0.33 & $11(12)$ & 0.57 \\
\hline
\end{tabular}

*Destinations for Network 3 are displayed between parentheses.

Table 4.6: Release-day (RD) distributions for all networks in the Benchmark Experiments

\begin{tabular}{|c|c|c|c|c|c|}
\hline \multicolumn{2}{|c|}{ Short } & \multicolumn{2}{|c|}{ Medium } & \multicolumn{2}{|c|}{ Long } \\
\hline $\mathrm{RD}$ & Prob. & $\mathrm{RD}$ & Prob. & $\mathrm{RD}$ & Prob. \\
\hline 0 & 0.6 & 0 & 0.33 & 0 & 0.1 \\
\hline 1 & 0.3 & 1 & 0.34 & 1 & 0.3 \\
\hline 2 & 0.1 & 2 & 0.33 & 2 & 0.6 \\
\hline
\end{tabular}


Table 4.7: Time-window (TW) length distributions for all networks in the Benchmark Experiments

\begin{tabular}{cccccccc}
\hline \multicolumn{2}{c}{ Short } & & \multicolumn{2}{c}{ Medium } & & \multicolumn{2}{c}{ Long } \\
TW & Prob. & & TW & Prob. & & TW & Prob. \\
\hline 4 & 0.6 & & 4 & 0.33 & & 4 & 0.1 \\
5 & 0.3 & & 5 & 0.34 & & 5 & 0.3 \\
6 & 0.1 & & 6 & 0.33 & & 6 & 0.6 \\
\hline
\end{tabular}

\section{4-D Absolute Results of the Benchmark Experiments}

Table 4.8: Average realized rewards for different time-window distributions

\begin{tabular}{|c|c|c|c|c|c|c|c|}
\hline \multirow{2}{*}{$\begin{array}{l}\mathrm{RD} \\
\text { distribution }\end{array}$} & \multirow{2}{*}{$\begin{array}{l}\text { TW } \\
\text { distribution }\end{array}$} & \multicolumn{2}{|c|}{ Network 1} & \multicolumn{2}{|c|}{ Network 2} & \multicolumn{2}{|c|}{ Network 3} \\
\hline & & $\mathrm{BH}$ & $\mathrm{ADP}$ & $\mathrm{BH}$ & $\overline{\mathrm{ADP}}$ & $\mathrm{BH}$ & $\mathrm{ADP}$ \\
\hline \multirow{3}{*}{ Short } & Short & 17,862 & 12,131 & 12,339 & 11,289 & 22,191 & (19) \\
\hline & Medium & 25,286 & 22,775 & 18,232 & 23,486 & 26,634 & 15,001 \\
\hline & Long & 33,007 & 35,111 & 25,805 & 32,524 & 30,680 & 29,745 \\
\hline \multirow{3}{*}{ Medium } & Short & 17,812 & 10,938 & 12,160 & 9,877 & 22,141 & 209 \\
\hline & Medium & 25,302 & 22,267 & 18,052 & 23,015 & 26,573 & 14,612 \\
\hline & Long & 32,805 & 34,508 & 25,420 & 31,806 & 30,473 & 29,502 \\
\hline \multirow{3}{*}{ Long } & Short & 17,773 & 10,724 & 12,062 & 9,281 & 22,256 & $(44)$ \\
\hline & Medium & 25,276 & 21,956 & 17,951 & 23,422 & 26,568 & 14,167 \\
\hline & Long & 32,876 & 34,511 & 25,401 & 32,462 & 30,467 & 29,274 \\
\hline
\end{tabular}

Table 4.9: Average realized rewards for different time-window distributions considering $R P 2$

\begin{tabular}{|c|c|c|c|c|c|c|c|}
\hline \multirow{3}{*}{$\begin{array}{l}\mathrm{RD} \\
\text { distribution }\end{array}$} & \multirow{3}{*}{$\begin{array}{l}\text { TW } \\
\text { distribution }\end{array}$} & \multicolumn{2}{|c|}{ Network 1} & \multicolumn{2}{|c|}{ Network 2} & \multicolumn{2}{|c|}{ Network 3} \\
\hline & & $\mathrm{BH}$ & $\mathrm{ADP}$ & $\mathrm{BH}$ & $\overline{\mathrm{ADP}}$ & $\mathrm{BH}$ & $\mathrm{ADP}$ \\
\hline & & $\mathrm{RP} 2$ & $\mathrm{RP} 2$ & $\mathrm{RP} 2$ & $\mathrm{RP} 2$ & $\mathrm{RP} 2$ & $\mathrm{RP} 2$ \\
\hline \multirow{3}{*}{ Short } & Short & 9,273 & 12,131 & 9,014 & 11,289 & 9,374 & (19) \\
\hline & Medium & 9,677 & 22,775 & 9,244 & 23,486 & 9,537 & 15,001 \\
\hline & Long & 10,151 & 35,111 & 9,601 & 32,524 & 9,728 & 29,745 \\
\hline \multirow{3}{*}{ Medium } & Short & 9,322 & 10,938 & 9,003 & 9,877 & 9,494 & 209 \\
\hline & Medium & 9,814 & 22,267 & 9,338 & 23,015 & 9,728 & 14,612 \\
\hline & Long & 10,341 & 34,508 & 9,719 & 31,806 & 9,881 & 29,502 \\
\hline \multirow{3}{*}{ Long } & Short & 9,438 & 10,724 & 9,074 & 9,281 & 9,601 & $(44)$ \\
\hline & Medium & 10,037 & 21,956 & 9,485 & 23,422 & 9,890 & 14,167 \\
\hline & Long & 10,643 & 34,511 & 9,944 & 32,462 & 10,150 & 29,274 \\
\hline
\end{tabular}




\section{4-E Benchmark Heuristic}

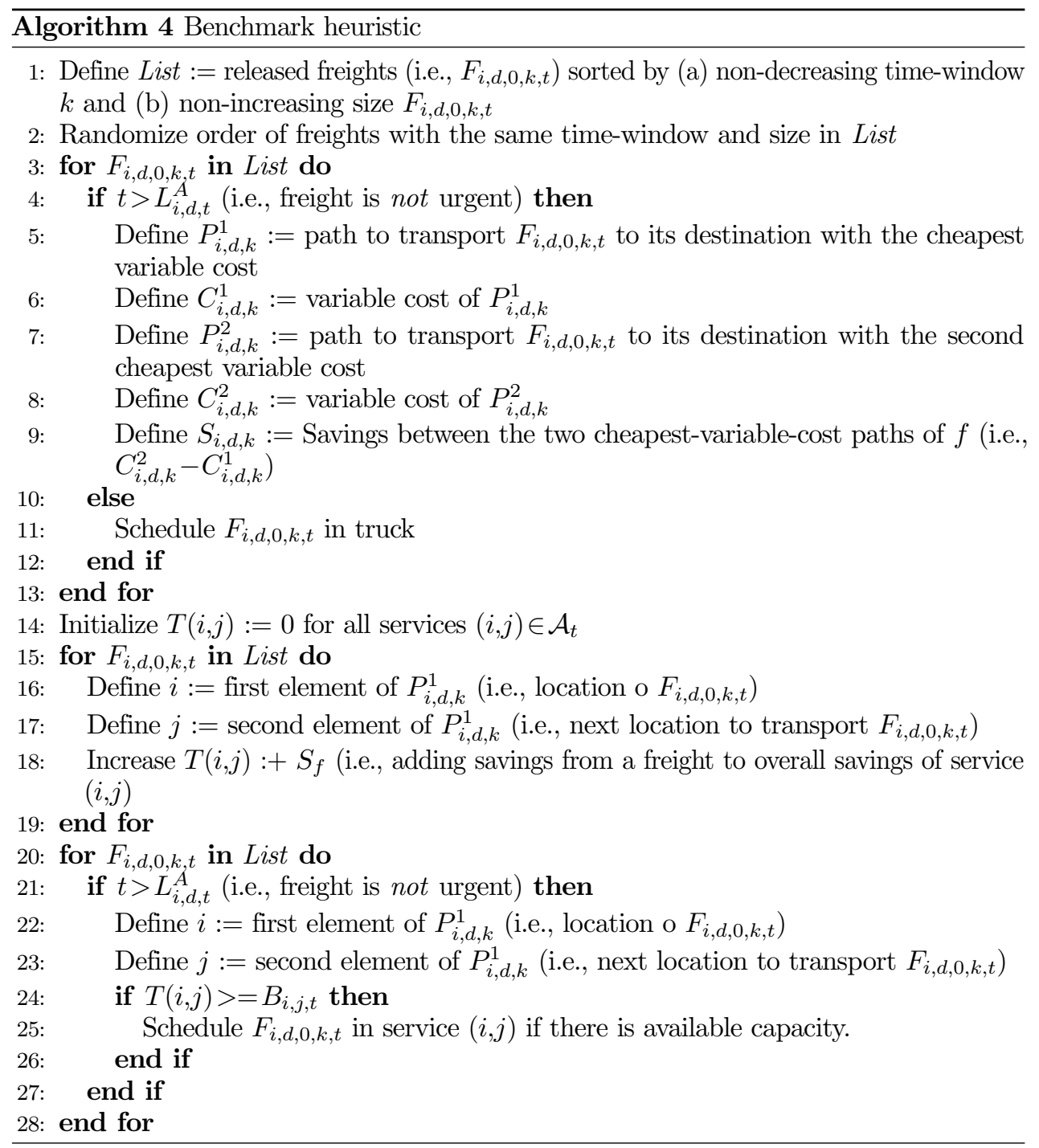

\section{4-F Different architectures for the VFA}

The success of any approximation method in ADP largely depends on finding a good set of representative variables of the downstream rewards. These variables, and their architecture, 
must capture the trade-offs in postponement of freight transport and in transfers of freight from one mode to another. Such architectures can be based on lookup-tables, such as feature clustering, state aggregation, state representatives, hierarchical aggregation, etc. Another way, closer to our basis functions approach is to include non-linear basis functions, as we further discuss in this appendix.

In our experiments, the set of basis functions $\mathcal{B}_{t}$ introduced in Section 4.5.1 contains variables related to freight characteristics only, i.e., time-windows and destinations. In Chapter 2, we observed that such relatively straightforward basis functions can capture the postponement (and consolidation) trade-off. However, they are less suitable for capturing the trade-off of transferring freight from one mode to anther in an intermediate terminal since other characteristics of the transport process (e.g., transport mode capacities) are not included. To capture this "transfer" trade-off, one could use variables that relate feasible freights (i.e., freights whose time-window can still be met even after a detour/transfer is realized) and transport modes characteristics such as capacity, service destination, etc. The following are three examples of such variables.

1. Transport under-capacity per terminal. These basis functions count the number of additional capacity required to transport all feasible (time-window wise) freights in each terminal. For example, Terminal 6 of Figure 4.4 has an outgoing capacity of 6 freights, meaning that if there are 4 feasible freights, the under-capacity is 0 , but if there are 7 feasible freights, the under-capacity is -1 .

2. Transport over-capacity per terminal. These basis functions count the number of excess capacity given the freights available at each terminal. For example, if in Terminal 6 of Figure 4.4 there are 4 feasible freights, the over-capacity is 2, but if there are 7 feasible freights, the over-capacity is 0.

3. 'Efficient' services per terminal. These basis functions count the number of services, at each terminal, whose setup may be covered by the difference between the variable costs of that service (plus trucking afterwards) and direct trucking of a set of feasible freights available. We discuss later on how the set of freights can be decided. This motivation for this variable is similar to the motivation of the benchmark heuristic described in Section 4.6.3.

The three features mentioned have a direct relation to the capacity of transport modes and may have a non-linear impact on the downstream rewards. Following the example of Terminal 6, low under capacity (say between 1 and 3 freights) may costs less per freight than a high under capacity (say more than 6 freights) since a few freights may be postponed and transported with the services of tomorrow rather than direct trucking. To model such differed impact, we could use a categorization of Under-Capacity (UC) into Low (L), Medium (M), and High (H), such as in Equations 4.25.

$$
f_{i, t}^{\mathrm{UC}}=\sum_{d \in \mathcal{N}_{t}^{\mathrm{D}}} \sum_{k \in \mathcal{K}_{t} \mid k<\Psi_{i}} \sum_{r \in \mathcal{R}_{t}^{\prime}} F_{i, d, r, k, t}^{x, n}, \quad \forall i \in \mathcal{N}_{t}^{\mathrm{I}}
$$




$$
\begin{gathered}
\phi_{i, t}^{\mathrm{UCL}}\left(S_{t}^{x, n}\right)=\left\{\begin{array}{ll}
f_{i, t}^{\mathrm{UC}}, & \text { if } 0>f_{i, t}^{\mathrm{UC}} \geq-3 \\
0, & \text { otherwise }
\end{array}, \quad \forall i \in \mathcal{N}_{t}^{\mathrm{I}}\right. \\
\phi_{i, t}^{\mathrm{UCM}}\left(S_{t}^{x, n}\right)=\left\{\begin{array}{ll}
f_{i, t}^{\mathrm{UC}}, & \text { if }-3>f_{i, t}^{\mathrm{UC}} \geq-6 \\
0, & \text { otherwise }
\end{array}, \quad \forall i \in \mathcal{N}_{t}^{\mathrm{I}}\right. \\
\phi_{i, t}^{\mathrm{UCH}}\left(S_{t}^{x, n}\right)=\left\{\begin{array}{ll}
f_{i, t}^{\mathrm{UC}}, & \text { if }-6>f_{i, t}^{\mathrm{UC}} \\
0, & \text { otherwise }
\end{array}, \quad \forall i \in \mathcal{N}_{t}^{\mathrm{I}}\right.
\end{gathered}
$$

The third feature, the number of services whose setup costs may be covered efficiently, depends on how freights are assigned to the transport modes at each terminal. This assignment could be solved using a MILP model or as a greedy heuristic (e.g., sorting services by non-increasing capacity and assigning freights whose destination is closest to the end-location of the service first). Independent of the assignment decision, when evaluating different post-decision states, a single terminal may result in the same number of efficient services for different freights. To capture which freights are better for a terminal to have for a given number of efficient services, one can divide the number of efficient services by the sum of distances of the end-location to the destination of freights consolidated. In this way, a post-decision state with a large value for this feature would be desirable since many services can be used with a good consolidation of freight.

Designing and evaluating a good approximation architecture for ADP based on basis functions is both a science and an art, as discussed in Chapter 2. In this chapter we exemplified how an advance learning mechanism can improve the performance of simple basis functions. With a better set of basis functions that better represent the different trade-offs in our problem, we can expect better performance, and possible faster learning, in ADP. Further research on the workings of ADP with different sets of features that tackle different problem trade-offs can result in more effective and efficient ADP designs. 

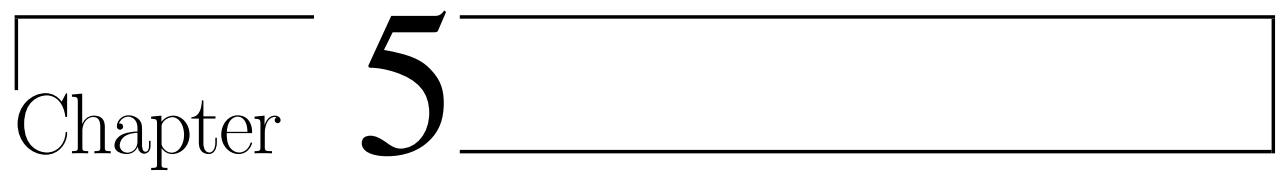

\section{Multi-terminal Drayage Transport}

In this chapter, we study the problem of scheduling drayage operations in synchromodal transport. Besides the usual decisions to time the pick-up and delivery of containers, and to route the vehicles that transport them, synchromodal transport includes the assignment of empty and loaded containers to terminal. The challenge consists of simultaneously deciding on these three aspects while considering various resource and timing restrictions. We model the problem using Mixed Integer Linear Programming (MILP) and design a matheuristic to solve it. Our algorithm iteratively confines the solution space of the MILP using several adaptations, and based on the incumbent solutions, guides the subsequent iterations and solutions. We test our algorithm under different problem configurations and provide insights into their relation to the three aspects of scheduling drayage operations in synchromodal transport. This chapter is based on our publication in [73].

\subsection{Introduction}

In previous chapters, we discussed how the scheduling flexibility due to synchromodality can bring efficiency benefits in long-haul transport, and we evaluated these benefits under various long-haul transport settings. However, the potential benefits discussed before depend to a great extent on the proper scheduling of drayage operations, also known as pre- and end-haulage or first and last-mile trucking. Drayage operations, which account for $40 \%$ of the total transport costs in a multi-modal transport chain [27], are the first step where the synchromodal flexibility in transport mode can be taken advantage of. In this chapter, we study the scheduling of drayage operations of multi-modal transport considering terminal assignment (i.e., assignment of the start of the long-haul) decisions. 
Drayage operations in multi-modal transport include delivery and pick-up requests of either empty or loaded containers, to and from a terminal where long-haul modes arrive and depart. These operations occur, for example, at a Logistic Service Provider (LSP) handling both import and export containers. The planner scheduling drayage operations must decide upon the time to fulfill each request and the route of the vehicles that will carry out all requests. In synchromodality, the planner must also decide to which terminal to bring a loaded container and to which terminal or customer to bring an empty container. All these decisions must be made simultaneously, considering constraints such as time-windows for requests, terminals, containers, trucks, and decoupling of requests for the delivery of an empty container and the subsequent pickup of a loaded one (and vice versa). Furthermore, re-scheduling the requests is allowed as new information becomes known (e.g., real-time information about requests, delays, etc.). In such a dynamic environment, making assignment, timing, and routing decisions together is difficult [27, 106]. Nonetheless, scheduling drayage operations with an integrated approach can bring significant savings [10].

The focus of this chapter is on Part 3 of the division of the synchromodal transport network presented in Figure 1.6 of Chapter 1. As in Chapters 2 and 4, which correspond to Part 1 and Part 2 of the synchromodal network division, our approach in this chapter is to formulate an exact model of the problem and subsequently design a heuristic algorithm to solve the model. Specifically, our goal is to develop a scheduling method to simultaneously make assignment, timing, and routing decisions for drayage operations. We categorize the drayage requests and analyze their implications in the aforementioned decisions. With our categorization, we identify challenges and opportunities for scheduling methods. In addition, we formulate the problem as a Mixed-Integer Linear Programming (MILP) model based on our categorization of requests and discuss possible improvements based on this categorization. We construct several adaptations to the MILP model and design a heuristic algorithm around them to schedule drayage operations and update the schedule as new requests arrive.

The remainder of this chapter is structured as follows. In Section 5.2, we briefly review the relevant literature and specify our contribution to it. In Section 5.3, we describe the problem in more detail and present our categorization of drayage requests. We introduce the notation and formulate the problem as a MILP in Section 5.4. In Section 5.5, we present our adaptations to the MILP model and our heuristic solution approach. In Section 5.6, we evaluate our heuristic solution approach through a series of numerical experiments, in a static and dynamic setting, and compare it to the use of a benchmark heuristic. In Section 5.7, we reflect upon the limitations of our approach. We finalize in Section 5.8 with conclusions.

\subsection{Literature Review}

We briefly review the literature about scheduling drayage operations in multi-modal transport. We examine the characteristics of the proposed models and study their applicability to our problem. For a more in-depth review, we refer the reader to [26], noticing that literature about scheduling drayage operations is scarce. We finalize this section by stating our contribution.

Most studies about scheduling drayage operations use mathematical programming. This technique allows researchers to model various problem characteristics at the price of high 
computational complexity. For this reason, researchers typically consider one problem characteristic at a time. For example, studies that consider more than one terminal, such as [66] and [10], assume a homogenous fleet. Studies that consider a flexible origin or destination for some requests, such as [12] and [35], consider only one terminal. Studies that do not assume a homogenous fleet, such as[45], avoid other constraints such as request time-windows. All in all, mathematical programming can relate various problem attributes to optimal decisions but requires further developments to handle the actual scheduling.

There is a variety of approaches available to solve the actual scheduling of drayage operations. There are sequential approaches, such as [12] and [66], that pair delivery and pickup customers before the routing. There are also integrated approaches, such as $[10$, $35,88,114]$, which handle paring (i.e, scheduling) and routing decisions simultaneously. Particularly, these integrated approaches show that a combination of parts of the mathematical problems with other heuristics perform well in solving the problem. Finally, the majority of approaches focuses on "one schedule" per day with no re-scheduling, except for [27], which re-schedules when the problem conditions change, and for [42], which re-schedules when new orders arrive, or when real-time information regarding traffic or position of trucks gives rise to this. Naturally, dynamic re-scheduling is another attribute of drayage operations that increases the complexity of the problem.

Although drayage operations contribute significantly to the total costs of multi-modal transport [48], research on these operations has been limited in modeling considerations and solution approaches [13]. The need for dynamic scheduling methods for multi-modal routing that take into account multiple attributes of the problem has been recognized [13]. For these reasons, our contribution to the literature is two-fold: (i) we model the scheduling of drayage operatiosn in synchromodal transport, and its multiple attributes, as a MILP and (ii) we design a matheuristic to solve the model.

\subsection{Problem Description}

We study the problem of scheduling drayage requests in a synchromodal network with the objective of minimizing routing and terminal (i.e., long-haul mode) assignment costs. There are three simultaneous decisions: (i) timing the execution of requests, (ii) routing the vehicles that carry out the requests, and (iii) assigning long-haul terminals (or customers) to the requests. These decisions are subject to the characteristics of the requests, available trucks, available containers, and terminals. Requests are characterized by customer location, type of truck (e.g., driver clearance, chasis, trailer, etc.), type of container (e.g., size, security, refrigeration, etc.), time-window, service (i.e., loading, unloading) time, and decoupling allowance. Trucks are characterized by start and end location, type, maximum working time, setup cost, and variable cost. Containers are characterized by location, type, and amount. Terminals are characterized by location, time-window, and an assignment cost that represents costs for using a certain long-haul mode, container storage, etc.

The terminal assignment cost and the various request attributes in synchromodality enrich the common drayage operations in multi-modal transport. To analyze this enrichment, we classify the requests into pre-haulage and end-haulage. In a pre-haulage request, an empty 
container is brought to a customer location and subsequently (after loading) brought to one of the long-haul terminals. In an end-haulage request, a loaded container is brought to a customer location and subsequently (after unloading) brought to a terminal for storage or to another customer who has a container-compatible pre-haulage request. Some of these requests allow decoupling, which means that a truck delivering an empty (or loaded) container does not need to wait for it to be loaded (or unloaded) and that another truck can pick up the loaded (empty) container later on. We refer to all possible pre- and end-haulage requests as jobs in the remainder of this chapter. We now elaborate on the job configurations.

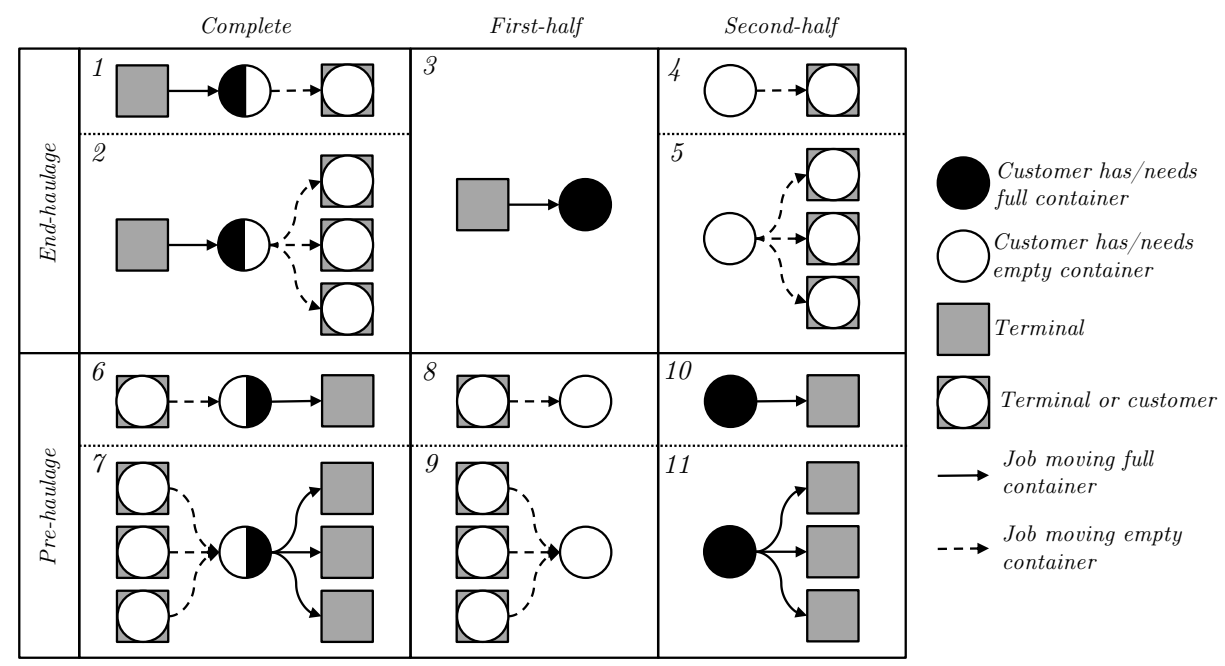

Figure 5.1: Possible job configurations in synchromodal transport

In drayage operations, there are various job configurations as seen in Figure 5.1. These configurations arise due to different contractual agreements, types of freight, types of resources, etc. In the complete job configurations of the end-haulage, the origin is a fixed terminal, but the destination can be either a given terminal (or customer) or one of multiple terminals (or customers), as seen in Types 1 and 2, respectively. If decoupling is allowed, the end-haulage job configurations can be divided into first- and second-half, as seen in Types 3,4 , and 5 . In the complete job configurations of the pre-haulage, the origin can be a given terminal (or customer) and the destination a given terminal, or the origin can be one of multiple terminals (or customers) and the destination one of multiple terminals, as seen in Types 6 and 7, respectively. Once more, if decoupling is allowed, the pre-haulage configurations can be divided into first- and second-half, as seen in Types 8, 9, 10, and 11.

Due to the full-truckload and multi-resource nature of the job configurations, some challenges and opportunities arise. For example, executing some job configurations after 
each other (e.g., Type 3 followed by Type 10) will require an empty movement of a truck, i.e., truck moving without a container. In another example, executing some job configurations after each other (e.g., Type 1 followed by Type 6) can allow the truck to skip the visit to a terminal (e.g., supersede the use of an empty container at a terminal). In such opportunities, some job configurations can be merged to reduce the decision complexity as also proposed by [45]. Using this job categorization, we formulate an MILP model that captures the challenges and opportunities in drayage operations in the following section. In the remainder of this chapter, when we talk about job types, we refer to the configurations seen in Figure 5.1.

\subsection{Mathematical Model}

Using the previous categorization of jobs, we construct two directed graphs $\mathcal{G}=(\mathcal{V}, \mathcal{A})$ and $\mathcal{G}^{\prime}=\left(\mathcal{V}, \mathcal{A}^{\prime}\right)$, which have the same nodes $\mathcal{V}$ but different $\operatorname{arcs} \mathcal{A}$ and $\mathcal{A}^{\prime}$. Nodes $\mathcal{V}$ represent all locations related to jobs and trucks: $\mathcal{V}=\mathcal{V}^{\mathrm{R}} \cup \mathcal{V}^{\mathrm{D}} \cup \mathcal{V}^{\mathrm{B}} \cup \mathcal{V}^{\mathrm{F}}$. Specifically, $\mathcal{V}^{\mathrm{R}}$ contains all job locations (i.e., request locations), $\mathcal{V}^{\mathrm{D}}$ all terminal locations, $\mathcal{V}^{\mathrm{B}}$ all beginning location of trucks and $\mathcal{V}^{\mathrm{F}}$ their finishing location. All nodes in $\mathcal{V}$ are indexed with $i$ and $j$. Arcs $\mathcal{A}$ and $\mathcal{A}^{\prime}$ are built to distinguish the assignment and the routing decisions, respectively. Arcs in $\mathcal{A}$ include all job-arcs between two nodes. Job-arcs are connections between nodes that comply with all resource and long-haul mode constraints. We define $\delta^{-}(r)=\{j:(j, r) \in \mathcal{A}\}$ and $\delta^{+}(r)=\{j:(r, j) \in \mathcal{A}\}$ as the sets of nodes that form job-arcs that are incoming to, and outgoing from, node $r \in \mathcal{V}^{\mathrm{R}}$, respectively. Arcs in $\mathcal{A}^{\prime}$ include all routing-arcs. These arcs follow a similar logic as in the VRP with time-windows formulation of [14] and are exemplified in a small numerical example in Appendix 5-A.

In $\mathcal{V}^{\mathrm{R}}$, each job is represented as a single node. In $\mathcal{V}^{\mathrm{D}}$, each terminal $d \in \mathcal{U}^{\mathrm{D}}$ is represented as $N_{d}$ identical nodes, in order to keep track of arrival times at $d$ in the model. The set $\mathcal{U}^{\mathrm{D}}$ is the set with unique terminal nodes. We index both sets with $d$. Each node $i \in \mathcal{V}^{\mathrm{R}} \cup \mathcal{V}^{\mathrm{D}}$ has a service time $S_{i}$ (i.e., time for loading, unloading, coupling, or decoupling a container), as well as a time-window described by an earliest arrival time $E_{i}$ and a latest arrival time $L_{i}$. In addition to $i$ and $j$, we index nodes in $\mathcal{V}^{\mathrm{R}}$ with $r$, noticing that $i$ and $j$ can represent any node whereas $r$ only represents nodes in $\mathcal{V}^{\mathrm{R}}$. For nodes $r \in \mathcal{V}^{\mathrm{R}}$, which represent jobs, $D_{r}$ gets a value of one if decoupling is allowed and zero otherwise. Traveling time between nodes $i$ and $j$ is denoted with $T_{i, j}$. Note that, for the identical nodes of each terminal, all time parameters are the same and traveling times between them are zero. Also note that two jobs can be at the same location, and thus traveling time between them is also zero. However, service times and time-windows can be different, depending on the job type.

To carry out all jobs, there is a fleet of heterogeneous trucks $\mathcal{K}$. The trucks that can carry out job $r \in \mathcal{V}^{\mathrm{R}}$ are represented with $\widetilde{K}(r) \subseteq \mathcal{K}$. Each truck begins its route in node $B_{k} \in \mathcal{V}^{\mathrm{B}}$ and finishes its route in node $F_{k} \in \mathcal{V}^{\mathrm{F}}$. All trucks have a maximum working time $T_{k}^{\mathrm{K}}$. Truck movements are modeled using the binary variable $x_{i, j, k}$, which gets a value of 1 if node $j$ is visited immediately after node $i$ by truck $k$, and 0 otherwise. Note that truck movements can either be to carry out a request (i.e., truck has an empty or loaded container) or to reposition the truck (i.e., no container). To model time in the movements of trucks, we use the auxiliary variable $w_{i}$, which represents the time at which the chosen truck arrives at node $i$. 
The goal is to perform all jobs, within their time-window, while minimizing routing and terminal assignment costs. To model the routing costs, we introduce (i) a fixed cost $C_{k}^{\mathrm{F}}$ for using truck $k \in K$ and (ii) a variable cost $C_{i, j, k}^{\mathrm{V}}$ for its movement over arc $(i, j) \in \mathcal{A}^{\prime}$. To model the terminal assignment costs, we introduce a cost $C_{r, d}^{\mathrm{D}}$ for assigning terminal $d \in \mathcal{V}^{\mathrm{D}}$ to job $r \in \mathcal{V}^{\mathrm{C}}$. Using the parameters and variables above, the optimization goal can be achieved solving the mathematical program shown in (5.1).

$$
\begin{aligned}
& \min z=\sum_{k \in \mathcal{K}}\left(C_{k}^{\mathrm{F}} \cdot \sum_{j \in \delta^{\prime}+\left(B_{k}\right)} x_{B_{k}, j, k}\right)+\sum_{k \in \mathcal{K}} \sum_{(i, j) \in \mathcal{A}^{\prime}} C_{i, j, k}^{\mathrm{V}} \cdot x_{i, j, k} \\
& +\sum_{k \in \mathcal{K}_{r} \in \mathcal{V}^{\mathrm{R}}} \sum_{d \in \delta^{+}(r) \cup \mathcal{V}^{\mathrm{D}}} C_{i, j}^{\mathrm{D}} \cdot x_{r, d, k} \\
& \text { s.t. } \\
& \sum_{k \in \widetilde{\mathcal{K}}(r)} \sum_{j \in \delta^{+}(r)} x_{r, j, k}=1, \forall r \in \mathcal{V}^{\mathrm{R}} \mid \delta^{+}(r) \neq \emptyset \\
& \sum_{k \in \widetilde{\mathcal{K}}(r)} \sum_{j \in \delta^{-}(r)} x_{j, r, k}=1, \forall r \in \mathcal{V}^{\mathrm{R}} \mid \delta^{-}(r) \neq \emptyset \\
& \left(1-D_{r}\right)\left(\sum_{j \in \delta^{+}(r)} x_{r, j, k}-\sum_{j \in \delta^{-}(r)} x_{j, r, k}\right)=0, \\
& \forall r \in \mathcal{V}^{\mathrm{R}} \mid \delta^{+}(r) \neq \emptyset \text { and } \delta^{-}(r) \neq \emptyset, k \in \widetilde{K}(r) \\
& \sum_{k \in \mathcal{K}} \sum_{j \in \delta^{\prime}+(r)} x_{r, j, k}=1, \forall r \in \mathcal{V}^{\mathrm{R}} \\
& \sum_{k \in \mathcal{K}} \sum_{j \in \delta^{\prime}+(d)} x_{d, j, k} \leq 1, \forall d \in \mathcal{V}^{\mathrm{D}} \\
& \sum_{j \in \delta^{\prime}+(i)} x_{i, j, k}-\sum_{j \in \delta^{\prime}-(i)} x_{j, i, k}=0, \forall i \in \mathcal{V}^{\mathrm{C}} \cup \mathcal{V}^{\mathrm{D}}, k \in \mathcal{K} \\
& E_{i} \leq w_{i} \leq L_{i}, \forall i \in \mathcal{V} \\
& \sum_{k \in \mathcal{K}}\left(x_{i, j, k} \cdot\left(w_{i}+S_{i}+T_{i, j}-w_{j}\right)\right) \leq 0, \forall i, j \in \mathcal{V} \\
& \sum_{k \in \mathcal{K}}\left(x_{B_{k}, j, k} \cdot T_{B_{k}, j}\right) \leq w_{j}, \forall j \in \mathcal{V} \\
& x_{i, F_{k}, k} \cdot\left(w_{i}+S_{i}+T_{i, F_{k}}-T_{k}^{\mathrm{K}}\right) \leq 0, \forall i \in \delta^{\prime}-\left(F_{k}\right), k \in \mathcal{K} \\
& \sum_{(i, j) \in \mathcal{A}^{\prime}} x_{i, j, k}-M_{k}^{\mathrm{A}} \cdot \sum_{j \in \delta^{\prime}+\left(B_{k}\right)} x_{B_{k}, j, k} \leq 0, \forall k \in \mathcal{K}
\end{aligned}
$$




$$
\begin{gathered}
\sum_{j \in \delta^{\prime}+\left(B_{k}\right)} x_{B_{k}, j, k} \leq M_{k}^{\mathrm{K}}, \forall k \in \mathcal{K} \\
\sum_{i \in \delta^{\prime}-\left(F_{k}\right)} x_{i, F_{k}, k}-\sum_{j \in \delta^{\prime}+\left(B_{k}\right)} x_{B_{k}, j, k}=0, \forall k \in \mathcal{K} \\
x_{i, j, k}=0, \forall i \in \mathcal{V}^{\mathrm{B}} \backslash\left\{B_{k}\right\}, j \in \mathcal{V}^{\mathrm{R}} \cup \mathcal{V}^{\mathrm{D}}, k \in \mathcal{K} \\
x_{i, j, k}=0, \forall i \in \mathcal{V}^{\mathrm{R}} \cup \mathcal{V}^{\mathrm{D}}, j \in \mathcal{V}^{\mathrm{F}} \backslash\left\{F_{k}\right\}, k \in \mathcal{K} \\
w_{i} \in \mathbb{R}, \forall i \in \mathcal{V} \\
x_{i, j, k} \in\{0,1\}, \forall i, j \in \mathcal{V}, k \in \mathcal{K}
\end{gathered}
$$

The objective is to minimize the total costs $z$ as shown in (5.1a). Constraints (5.1b) state that only one incoming job-arc can be used for job $r$. Note that it is possible that job $r$ does not require incoming job-arcs (e.g., Type 10), and thus $\delta^{+}(r)=\emptyset$. Similarly, (5.1c) ensure that only one outgoing job-arc can be used for job $r$. For jobs that have both incoming and outgoing job-arcs (i.e., Types 1, 2, 6, and 7), (5.1d) ensure that the same vehicle does both the incoming and outgoing job-arc if decoupling is not allowed for job $r$. Constraints (5.1e) ensure that all jobs $r \in \mathcal{V}^{\mathrm{R}}$ are carried out by one truck only. Constraints (5.1f) ensure that all terminal nodes $d \in \mathcal{V}^{\mathrm{D}}$ are visited at most once. Remind that a terminal has duplicate nodes for keeping track of time, meaning that the same terminal might be visited multiple times (e.g., for different jobs) but each time to a different duplicated node. Constraints (5.1g) ensure flow conservation, meaning that all nodes that are exited must be entered as well. The timewindows of jobs, terminals, and truck locations are enforced in (5.1h). Constraints (5.1i) and (5.1j) keep track of the time variables. The maximum working time of trucks is guaranteed by $(5.1 \mathrm{k})$. Constraints $(5.1 \mathrm{l})$ and $(5.1 \mathrm{~m})$ establish that each truck can only depart once from its starting location if it is used for doing jobs. In (5.11), $M_{k}^{\mathrm{A}}$ works as a "big-M" parameter that can be initialized, for example, with $M_{k}^{\mathrm{A}}=\left|\mathcal{V}^{\mathrm{R}}\right|+\left|\mathcal{V}^{\mathrm{D}}\right|+1$. However, it can also be used to restrict the number of routing-arcs that vehicle $k$ can traverse, as we will explain in Section 5.5. In a similar way, the auxiliary parameter $M_{k}^{\mathrm{K}}$ can be used to restrict the use of vehicle $k$ by setting $M_{k}^{\mathrm{K}}=0$. Initially, we set $M_{k}^{\mathrm{K}}=1, \forall k \in K$. Constraints (5.1n) state that each truck must end at its ending location if it has departed from its beginning location. Since the nodes $\mathcal{V}^{\mathrm{B}}$ and $\mathcal{V}^{\mathrm{F}}$ are used for modeling beginning and ending locations of trucks (i.e., not for carrying out jobs), we have to ensure that trucks do not visit them in any case, as shown in (5.1o) and (5.1p). Finally, Constraints (5.1q) and (5.1r) establish the domains of the variables.

Although the formulation above is not linear due to $(5.1 \mathrm{i})$ and $(5.1 \mathrm{k})$, we can linearize it by substituting these two with (5.2a) and (5.2b). The idea behind this linearization is that, if node $i$ precedes node $j$ in any route, the arrival time at node $j$ happens after the truck arrived at $i$, was served at $i$, and traveled from $i$ to $j$. Further explanation on the logic behind these constraints can be found in [12].

$$
\begin{array}{r}
w_{i}+S_{i}+T_{i, j}-\left(L_{i}+S_{i}+T_{i, j}-E_{j}\right) \cdot\left(1-\sum_{k \in \mathcal{K}} x_{i, j, k}\right) \leq w_{j} \forall i, j \in \mathcal{V} \\
w_{i}+S_{i}+T_{i, F_{k}}-\left(L_{i}+S_{i}+T_{i, F_{k}}\right) \cdot\left(1-x_{i, F_{k}, k}\right) \leq L_{F_{k}}, \forall i \in \delta^{\prime}-\left(F_{k}\right), k \in \mathcal{K}
\end{array}
$$


Our MILP formulation models the jobs as arcs that need to be traversed by the trucks. Another option to represent jobs in drayage operations is to model them as nodes. Modeling jobs as nodes reduces the size of the graph if some of the nodes are fixed beforehand [10]. However, flexible jobs (such as ours with the terminal assignment) cannot be collapsed into a single-node [88] and the gains of an integrated approach are harder to obtain when modeling jobs as nodes [10]. Although modeling jobs as arcs come with the price of a larger graph, there are other opportunities to improve the formulation through valid inequalities and pre-processing, as we describe in the following section.

\subsubsection{Valid Inequalities and Pre-Processing}

Due to the full-truckload nature of our problem, all jobs deal with at most one terminal either as the origin or the destination of a container. This means that a truck carrying out jobs will never visit more than two terminals consecutively. Since we model the requests and terminals as separate nodes, this means that not all arcs between terminal nodes will be traversed. An arc between two terminal nodes will only be traversed when delivering a container to the first one, and picking up a container from the second one. Thus, we can use a bound $M^{\mathrm{DE}}$ on them as shown in (5.3).

$$
\begin{gathered}
\sum_{k \in \mathcal{K}} \sum_{i \in \mathcal{V}^{\mathrm{D}}} \sum_{j \in \mathcal{V}^{\mathrm{D}}} x_{i, j, k} \leq M^{\mathrm{DE}} \\
M^{\mathrm{DE}}=\sum_{r \in \mathcal{V}^{\mathrm{R}}} \sum_{d \in \mathcal{U}^{\mathrm{D}}} B_{r, d} \mid B_{r, d}= \begin{cases}1 & \text { if } d \in \delta^{-}(r) \\
0 & \text { otherwise }\end{cases}
\end{gathered}
$$

In addition to the bound on the number of arcs between all terminal nodes $\mathcal{V}^{\mathrm{D}}$, we can bound the traversed arcs between replicated nodes of a terminal using a similar logic. We define the set $\mathcal{V}_{d}^{\mathrm{DR}} \subseteq \mathcal{V}^{\mathrm{D}}$ as the set containing all duplicated nodes of terminal $d \in \mathcal{U}^{\mathrm{D}}$. We put a bound $M_{d}^{\mathrm{DI}}$ on each unique terminal node $d \in \mathcal{U}^{\mathrm{D}}$ as shown in (5.4).

$$
\begin{gathered}
\sum_{k \in \mathcal{K}_{i}} \sum_{i \in \mathcal{V}_{d}^{\mathrm{DR}}} \sum_{j \in \mathcal{V}_{d}^{\mathrm{DR}}} x_{i, j, k} \leq M_{d}^{\mathrm{DI}}, \forall d \in \mathcal{U}^{\mathrm{D}} \\
M_{d}^{\mathrm{DI}}=\sum_{r \in \mathcal{V}^{\mathrm{R}}} \sum_{i \in \mathcal{V}_{d}^{\mathrm{DR}}} B_{r, i} \mid B_{r, i}=\left\{\begin{array}{ll}
1 & \text { if } i \in \delta^{-}(r) \\
0 & \text { otherwise }
\end{array}, \forall d \in \mathcal{U}^{\mathrm{D}}\right.
\end{gathered}
$$

Taking advantage that our problem deals with jobs that have at most one origin and at most one destination, we can compute a minimum traveling distance and traveling time to fulfill all jobs by choosing the origin and destination with the shortest distance and time, respectively. Using this information, we can calculate the minimum number $M^{\mathrm{LK}}$ of trucks needed (since trucks have a maximum working time) and a lower bound on the routing costs $M^{\mathrm{LC}}$. Furthermore, using a constructive heuristic (e.g., the one we benchmark to in Section 5.6), we can find upper bounds $M^{\mathrm{UK}}$ and $M^{\mathrm{UC}}$ for the number of trucks needed 
and the routing costs, respectively. Thus, we can limit the number of trucks as shown in (5.5) and the routing costs as shown in (5.6).

$$
\begin{gathered}
M^{\mathrm{LK}} \leq \sum_{k \in K} \sum_{j \in \delta^{\prime}+\left(B_{k}\right)} x_{B_{k}, j, k} \leq M^{\mathrm{UK}} \\
M^{\mathrm{LC}} \leq \sum_{k \in K}\left(C_{k}^{F} \cdot \sum_{j \in \delta^{\prime}+\left(B_{k}\right)} x_{B_{k}, j, k}\right)+\sum_{k \in K} \sum_{(i, j) \in \mathcal{A}^{\prime}} C_{i, j, k}^{\mathcal{V}} \cdot x_{i, j, k} \leq M^{\mathrm{UC}}
\end{gathered}
$$

The last potential improvement we introduce is the pre-processing of time-windows. In our model, there are duplicated nodes (i.e., same location, service time, and time-window) for each terminal to keep track of time. However, each duplicated terminal node can only be used for one job. Since we duplicate a terminal for each job that might use that terminal, we can use the time-window of the job to reduce the time-window of the duplicated node for that terminal. As an example, consider Figure 5.2. In this figure, we see a job of Type 1 that requires a full container from terminal $d$ and delivers an empty container to terminal $d^{\prime}$. In order to carry out this job within its time-window $\left[E_{r}, L_{r}\right]$, the full container must be put on a truck and travel from terminal $d$ anywhere between $\left[E_{r}-\left(S_{d}+T_{d, r}\right), L_{r}-\left(S_{d}+T_{d, r}\right)\right]$. Similarly, after unloading the container, the empty container can arrive to terminal $d^{\prime}$ anywhere between $\left[E_{r}+\left(S_{r}+T_{r, d^{\prime}}\right), L_{r}+\left(S_{r}+T_{r, d^{\prime}}\right)\right]$. We can repeat this logic for all jobs, their associated (possible) terminals, and the duplicated nodes for those terminals.

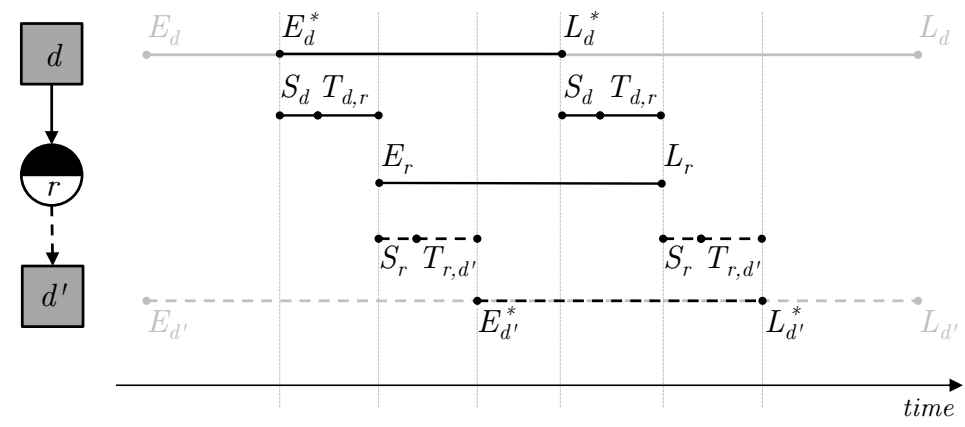

Figure 5.2: Example of pre-processing of time-windows for a job Type 1

The benefit of the aforementioned enhancements of the MILP is twofold. First, the valid inequalities tighten the feasible solution space (see example in Appendix 5-A). Second, the time-window pre-processing breaks the symmetry in MILP solutions introduced by the duplicated terminal nodes. However, these modifications are sufficient to solve only small problems. In the following section, we elaborate on further adaptations to the MILP that allow it to be applied to larger problems. 


\subsection{Solution Algorithm}

In our problem, MILP solvers are able to find a good feasible solution fast, but struggle on improving it further or in proving its optimality. In this section, we design three adaptations to the MILP that are aimed to help a solver find good feasible solutions faster. Furthermore, we design two matheuristics: (i) a static matheuristic to solve a single instance of the problem using Math-Heuristic Operators (MHOs), and (ii) a dynamic matheuristic to solve a re-scheduling instance of the problem using Fixing Criteria (FCs). We now elaborate on the MHOs, FCs, and the workings of each algorithm.

\subsubsection{Static Matheuristic}

Our static matheuristic uses three adaptations to the MILP, iteratively and in a local-search fashion, as shown in the pseudo-code of Algorithm 5. These adaptations, denoted by MHOs, are basically additional constraints in the MILP that can be seen as cutting planes that reduce the feasible space. Since our formulation results in a lot of arcs, our MHOs focus on fixing those arcs in an intuitive way. We now explain each MHO in more detail.

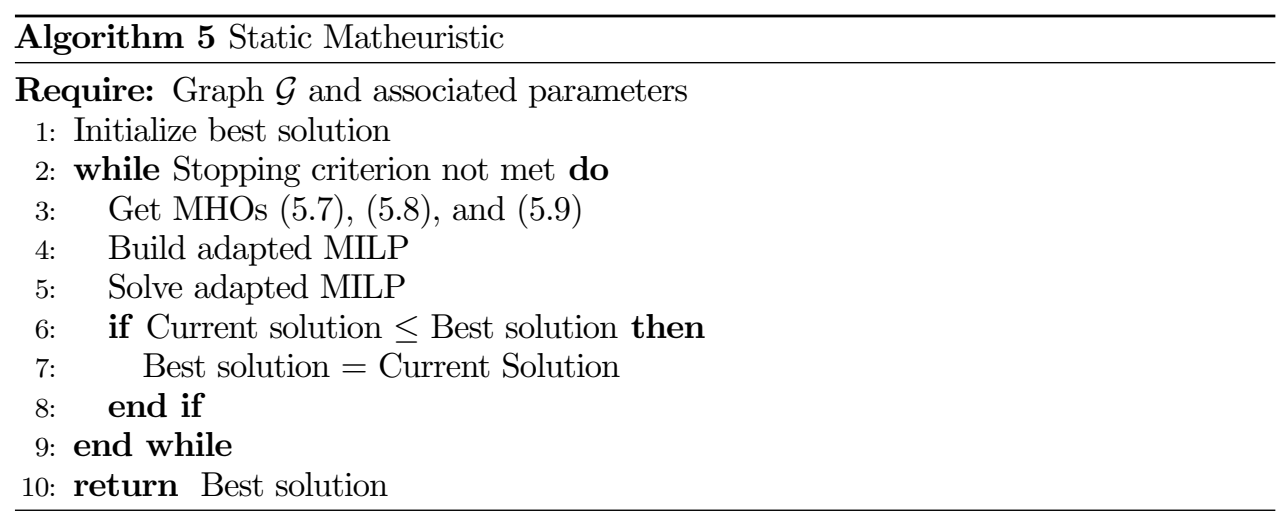

MHO 1: For $N^{\mathrm{M} 1}$ random jobs $r \in \mathcal{V}^{\mathrm{R}}$, we limit the number of feasible job-arcs to at most two, i.e., $\left|\delta^{-}(r)\right| \leq 2$ and $\left|\delta^{+}(r)\right| \leq 2$. These arcs are from (or to) the two closest locations (i.e., shortest traveling time). In other words, all remaining job-arcs are cut out, as shown in (5.7).

$$
\begin{aligned}
& x_{j, r, k}=0, \forall k \in \mathcal{K}, j \in \delta^{-}(r) \backslash\left\{i, i^{\prime}\right\} \mid i=\underset{j \in \delta^{-}(r)}{\operatorname{argmin}} T_{j, r} \text { and } i^{\prime}=\underset{j \in \delta^{-}(r) \backslash\{i\}}{\operatorname{argmin}} T_{j, r} \\
& x_{r, j, k}=0, \forall k \in \mathcal{K}, j \in \delta^{+}(r) \backslash\left\{i, i^{\prime}\right\} \mid i=\underset{j \in \delta^{+}(r)}{\operatorname{argmin}} T_{r, j} \text { and } i^{\prime}=\underset{j \in \delta^{+}(r) \backslash\{i\}}{\operatorname{argmin}} T_{r, j}
\end{aligned}
$$

MHO 2: For $N^{\mathrm{M} 2}$ times, the arc between a job $r$ of Type 2 and a job $r^{\prime}$ of Type 7 with the minimum traveling time is fixed. Remind that the arc is feasible when $r \in \delta^{-}\left(r^{\prime}\right)$ and 
$r^{\prime} \in \delta^{+}(r)$, and thus the fixing of a pair of jobs $r$ and $r^{\prime}$ can be done as shown in (5.8).

$$
\sum_{k \in \mathcal{K}} x_{r, r^{\prime}, k}=1 \mid r=\underset{j \in \delta^{-}\left(r^{\prime}\right)}{\operatorname{argmin}} T_{j, r^{\prime}}
$$

MHO 3: For $N^{\mathrm{M} 3}$ random jobs $r \in \mathcal{V}^{\mathrm{R}}$, we fix the feasible job-arcs from (or to) the closest location (i.e., shortest traveling time), as shown in (5.9).

$$
\sum_{k \in \mathcal{K}} x_{i, r, k}=1 \mid i \underset{j \in \delta^{-}(r)}{\operatorname{argmin}} T_{j, r} \quad \text { and } \quad \sum_{k \in \mathcal{K}} x_{r, i, k}=1 \mid i \underset{j \in \delta^{+}(r)}{\operatorname{argmin}} T_{r, j}
$$

Assigning a value to the parameters $N^{\mathrm{M}^{*}}$ requires tuning (see Section 5.6.2). In general, the larger the value of $N^{\mathrm{M}^{*}}$ the smaller the problem becomes for the solver, but the higher the chance of ruling out the global optimum.

\subsubsection{Dynamic Matheuristic}

Our dynamic matheuristic builds upon the static and is used for re-scheduling situations (e.g., new jobs arrived, delays, etc.). Jobs sequences that are being executed during the rescheduling trigger are completed (i.e., no preemption). For jobs that have not been started yet, we have the option to use the previous schedule or to re-schedule them. We design a matheuristic that takes advantage of both options, as shown in the pseudo-code of Algorithm 6 .

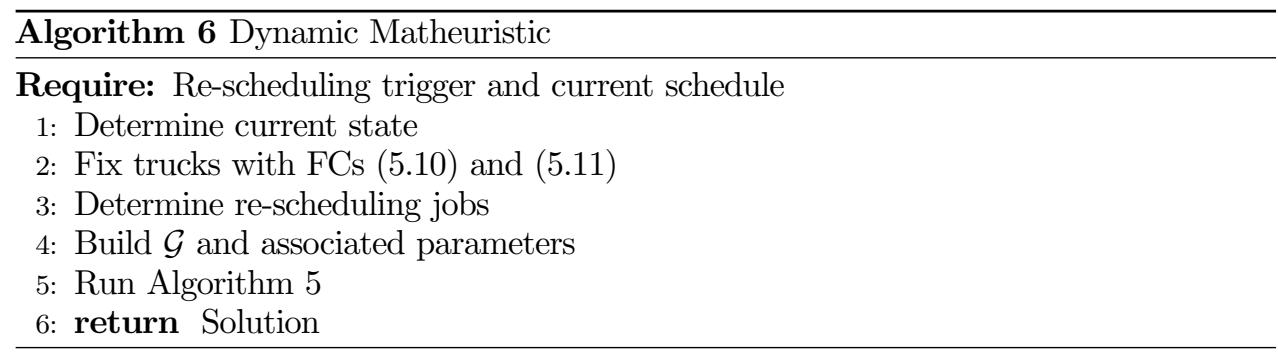

First, the matheuristic determines the current state, i.e., which jobs are completed, being executed, or not started yet, and which characteristics do the current truck routes have. Second, the matheuristic uses two Fixing Criteria (FC) to fix the job sequence of some routes, but leave the trucks that will execute these routes, as well as the arrival time to each job, open for re-scheduling. The idea of these fixing criteria is to identify good routes from the current schedule that can be kept in the new schedule but also to allow flexibility to add new jobs and to handle delays in routes and trucks already in use. Third, the dynamic matheuristic uses the static matheuristic to build a new scheduled based on the fixing criteria and the new information (e.g., new jobs, delays, etc.). We now explain each FC 
in more detail.

FC 1: We fix $N^{\mathrm{F} 1}$ routes $k^{\mathrm{C}}$ from the current schedule $x^{\mathrm{C}}$ with the largest number of jobs. We define $\mathcal{F}^{1}\left(k^{\mathrm{C}}\right)$ as the set of $\operatorname{arcs}(i, j)$ that fulfill this criteria, as shown in (5.10), and fix the routes by $\sum_{k \in \mathcal{K}} x_{i, j, k}=1, \forall(i, j) \in \mathcal{F}^{1}\left(k^{\mathrm{C}}\right)$.

$$
\mathcal{F}^{1}\left(k^{\mathrm{C}}\right)=\left\{(i, j) \in \mathcal{A}: x_{i, j, k^{\mathrm{C}}}^{\mathrm{C}}=1, k^{\mathrm{C}}=\underset{k^{\prime} \in \mathcal{K} \mid \sum_{j \in \mathcal{V}^{-}}^{\mathrm{C}} \mathrm{B}_{k}, j, k}{\operatorname{argmax}} \sum_{i \in \mathcal{V}^{\mathrm{R}}} x_{i, j, k}^{\mathrm{C}}\right\}
$$

FC 2: We fix $N^{\mathrm{F} 2}$ routes with the shortest traveling time, similar to FC 1. We define $\mathcal{F}^{2}\left(k^{\mathrm{C}}\right)$ as the set of $\operatorname{arcs}(i, j)$ that fulfill this criteria, as shown in (5.11).

$$
\mathcal{F}^{2}\left(k^{\mathrm{C}}\right)=\left\{(i, j) \in \mathcal{A}: x_{i, j, k^{\mathrm{C}}}^{\mathrm{C}}=1, k^{\mathrm{C}}={\underset{k}{k^{\prime} \in \mathcal{K} \mid \sum_{j \in \mathcal{V}^{x}}^{\mathrm{C}} \operatorname{Br}_{k}, j, k}=1}_{(i, j) \in \mathcal{A}^{\prime}}^{\operatorname{argmin}} x_{i, j, k}^{\mathrm{C}} T_{i, j}\right\}
$$

Just as in the static matheuristic, the best value of the parameters $N^{\mathrm{F}^{*}}$ depends on circumstances such as the current schedule, the instance $\mathcal{G}$, and the re-scheduling trigger. In the following, we present a brief proof-of-concept of the algorithms just described.

\subsection{Numerical Experiments}

We test our solution approach in two numerical experiments. First, we examine the benefits that our adaptations have in solving the MILP. Second, we test the gains of using our dynamic matheuristic compared to a benchmark heuristic. Our goal is to explore our approach and gain insights for further research.

\subsubsection{Experimental Setup}

We design 32 problem instances containing 25 jobs each. The location, time-window, and service time for each job are obtained from the first 25 customers of several Solomon instances for the VRPTW [90]. We use the first eight instances of four categories in [90]: C1, $\mathrm{C} 2$, R1, and R2, where $\mathrm{C}$ stands for clustered locations, $\mathrm{R}$ for random locations, 1 for short time-windows, and 2 for long-time windows. For each instance, there are 25 homogeneous trucks to guarantee there is a feasible solution. For each truck, the fixed cost is 1000 and the traveling time and variable cost are equal to the Euclidean distance between two locations.

The job configurations for all instances are shown in Table 5.1. These job configurations are based on the average drayage operations of a Dutch LSP in the Eastern part of The Netherlands. This LSP has three terminals that we use as follows. Terminal 1 is located in the same location as the Depot in the corresponding Solomon instance and has a terminal assignment cost of 500. Terminal 2 and Terminal 3 are located at $(60,60)$ and $(10,10)$, which are points along the diagonal in the Euclidean space that are close to the most distant 
customers from the geographical center of each of the Solomon instances. The assignment costs for Terminal 2 and 3 vary per instance, and are defined as $500-2 \beta$, where $\beta$ differs per instance and is defined as the length of the diagonal formed by the leftmost bottom location and the rightmost top location in that instance. The rationale behind Terminals 2 and 3 having a lower terminal assignment cost than Terminal 1 is to make the distant terminals worth "assigning", i.e., it can be cheaper to go to a distant terminal despite the larger travel distances. The time-window of each terminal is twice the time-window of the depot in the corresponding Solomon instance. The maximum working time for each truck is equal to the length of the time-window of each terminal. Terminal 1 is the beginning and finishing location of nine trucks, and Terminals 2 and 3 of eight trucks each.

Table 5.1: Job configuration for all instances

\begin{tabular}{lccccccccccc}
\hline \multirow{2}{*}{ Characteristic } & \multicolumn{11}{c}{ Job Type } \\
\cline { 2 - 12 } & 1 & 2 & 3 & 4 & 5 & 6 & 7 & 8 & 9 & 10 & 11 \\
\hline Number of jobs & 2 & 3 & 2 & 2 & 2 & 2 & 4 & 3 & 3 & 1 & 1 \\
Jobs decoupling $D_{r}$ & 1 & 1 & - & - & - & 1 & 1 & 1 & - & - & - \\
\hline
\end{tabular}

In both the static and dynamic experiments, we compare to the use of a benchmark heuristic that follows the logic from [12]. First, each job of Type 2 is paired with a job of Type 7 that incurs the minimum variable cost considering all constraints. Note that the origin of the full container of Type 2 is known, thus the pairing occurs in the destination of the empty container of Type 2 with the source of the empty container of Type 7. Subsequently, the closest terminal is assigned to the full container of Type 7. The paired jobs are sorted in non-decreasing route distance and scheduled using a cheapest insertion method. This method schedules the paired jobs in the position of the route that yields the lowest routing cost. All remaining jobs are then scheduled with a similar cheapest insertion method. For the jobs that have a flexible source or destination of a container, all combinations of sources and destinations are examined and the position with the cheapest routing and terminal assignment cost is chosen. To use this heuristic dynamically, the job sequences that are being executed during the re-scheduling trigger are fixed (i.e., no preemption of the current schedule). Jobs that have not been started and that are not in a non-preemptive sequence are re-scheduled with the steps described before. We now describe each experiment in detail and present their results.

\subsubsection{Static Experiments}

In the static experiments, we test the effect that the Valid Inequalities (VIs), Time-Window Pre-Processing (TWPP), and the three Math-Heuristic Operators (MHOs) have on the total costs. For each of the eight instances in categories $\mathrm{C} 1, \mathrm{C} 2, \mathrm{R} 1$, and R2, we test the MILP without any modification, the MILP with the VIs, the MILP with the TWPP, and the MILP with the VIs, TWPP, and each of the MHOs. We use CPLEX 12.6.3 (via the C API) with a limit of 300 seconds and a warm-start given by the solution of the Benchmark 
Heuristic (BH). For MHO 1 and 3, we perform nine iterations; and at each iteration, we choose seven random jobs in MHO 1 and one random job in MHO 3. MHO 2 has no randomness, so we perform only three iterations and we fix 1,2 , and 3 jobs respectively. The "random" settings are arbitrary, since we just test their usefulness rather than tuning them. We show the aggregated results in Table 5.2.

Table 5.2: Total costs for various MILP adaptations

\begin{tabular}{lrrrrrrr}
\hline Instances & BH & MILP & VIs & TWPP & MHO 1 & MHO 2 & MHO 3 \\
\hline C1 & 77,960 & 77,926 & 77,960 & 76,924 & 76,829 & 77,926 & 75,189 \\
C2 & 52,904 & 52,882 & 52,904 & 52,049 & 51,841 & 52,078 & 50,802 \\
R1 & 111,087 & 111,078 & 110,904 & 107,649 & 107,254 & 107,647 & 107,736 \\
R2 & 50,500 & 50,435 & 50,500 & 50,497 & 50,255 & 50,500 & 50,378 \\
\hline
\end{tabular}

In Table 5.2, four interesting observations arise. First, the VIs do not improve the solution of the MILP but the TWPP does. Second, the best solutions in the clustered (C) instances are achieved by MHO 3, and in the random $(\mathrm{R})$ instances by MHO 1. It is reasonable that MHO 1 achieves better results in $\mathrm{R}$ instances than $\mathrm{C}$ ones because this operator increases the chance of assigning the closest origin and destination to each job in a network with more disperse locations. Third, MHO 2 has worse solutions than the other MHOs. It seems that choosing a job as the origin/destination of an empty container (i.e., logic of the $\mathrm{BH}$ ) is not better than allowing a job or a terminal to be origin and destination, as MHO 1 and 3 allow. Fourth, in instances $\mathrm{C} 1, \mathrm{C} 2$, and R1, our adaptations to the MILP result in savings (from the $\mathrm{BH}$ ) between $3-4 \%$, but in instances $\mathrm{R} 2$, savings are relatively minor. It seems that in R2, which has long time-windows and longer traveling times, the cheapest insertion and job-pairing nature of the $\mathrm{BH}$ already results in good solutions.

\subsubsection{Dynamic Experiments}

In the dynamic experiments, we test the effect of the Fixing Criteria FC 1 and FC 2. In addition, we test a no-fixing criteria FC 0 meaning that all non-preemptive jobs can be re-scheduled. The problem instances are similar to the static experiments. We consider five stages for re-scheduling after the initial scheduling of the first 25 jobs of each Solomon instance (i.e., the static setup). These five stages are uniformly distributed within the first half of the trucks and terminals maximum working time (i.e., half a day). At each stage, five new jobs are revealed, which correspond to the next five jobs in each Solomon instance, and whose time-window is increased proportionally to the stage to guarantee they occur after they are revealed. Non-preemptiveness applies to all jobs scheduled in a truck before the next stop at a terminal. For the static matheuristic within the dynamic matheuristic, we use MHO 3 for $\mathrm{C}$ instances and MHO 1 for R instances. We define the number of truck routes to fix for $\mathrm{FC} 1$ and $\mathrm{FC} 2$ as a percentage of the available truck routes: $N^{\mathrm{F}^{*}}=(0.1,0.5)$. The results are shown in Figure 5.3 and 5.4. 


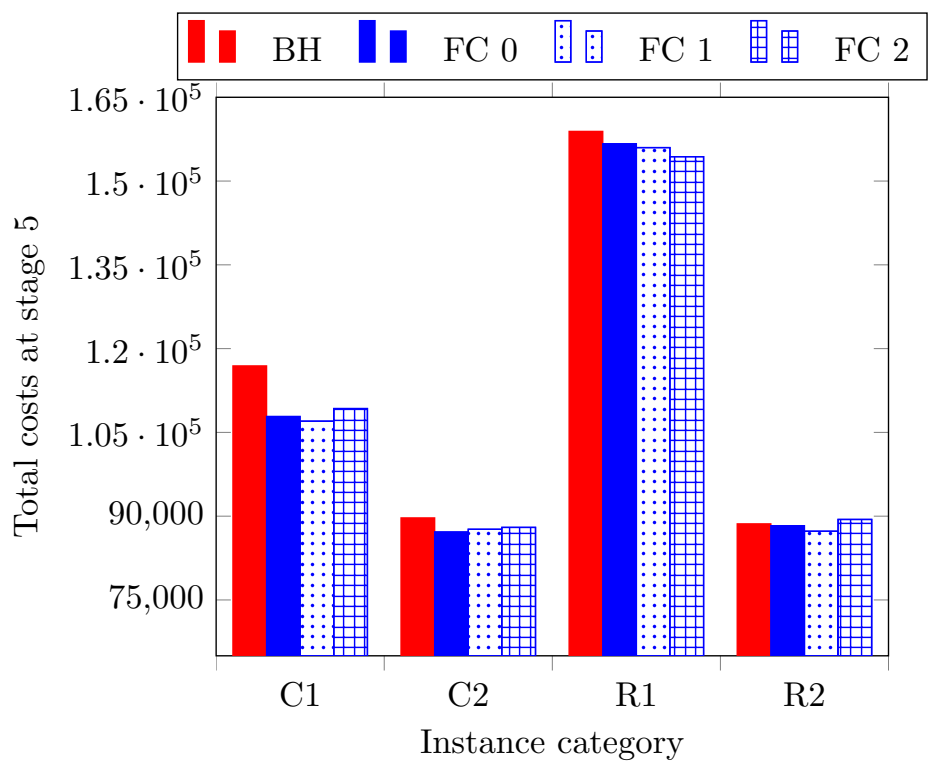

Figure 5.3: Comparison FCs at last stage

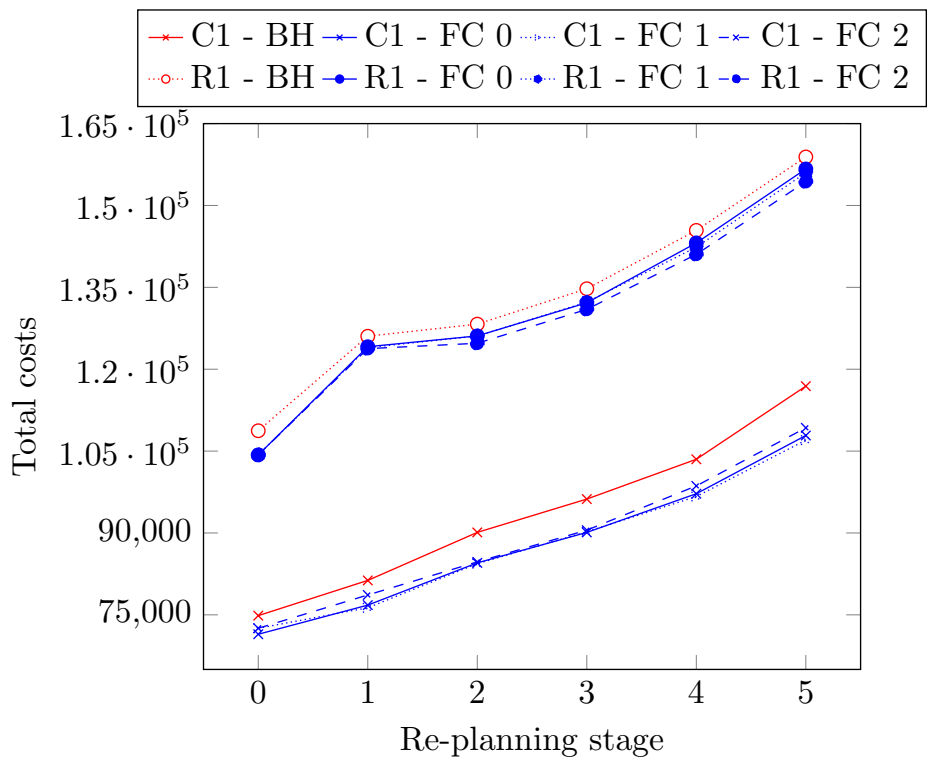

Figure 5.4: Performance of best FC per stage 
In Figure 5.3, we observe that after the last re-planning stage, our dynamic matheuristic achieves significant savings in total costs compared to the $\mathrm{BH}$ in instances $\mathrm{C} 1, \mathrm{C} 2$ and $\mathrm{R} 1$ (around 8\%, 3\%, and 3\% respectively). The winning FC, however, seems to vary per instance type. It is reasonable that fixing routes with the largest number of jobs (i.e., FC 1) is good in $\mathrm{C} 1$ since these instances contain closely located jobs with tight time-windows. Furthermore, it seems also reasonable that in instances with disperse located jobs such as R1, fixing routes with good traveling times (i.e., FC 2) is better. We focus on these two instance categories in Figure 5.4. For R1 we observe that FCs 0 and 1 have similar performance and that FC 2 starts differentiating more from the other FCs and the $\mathrm{BH}$ towards the last stages. For C1 we observe similarly that FCs 0 and 1 have comparable performance, and that the gap to the $\mathrm{BH}$ seems to widen towards the last stages.

\subsection{Discussion}

In the static experiments, we observe that the MHOs help obtaining a better MILP solution than the VIs and the TWPP. We also observe that the performance of the MHO depends on the problem settings. In the dynamic experiments, we observe that the dynamic matheuristic outperforms the $\mathrm{BH}$ but the performance of the FCs therein depends again on the problem settings. Although these experiments serve as a proof-of-concept and give an indication of the gains to be expected, they have three limitations. First, we do not tune the parameters with respect to the problem setting. As described in the results, our MHOs and FCs influence the distance traveled by some trucks, as well as the time of arrival to some jobs. The selection of these trucks and jobs requires tuning. Second, we do not adapt the algorithms towards previous iterations or stages. Our MHOs and FCs are analogous to neighborhood operators in local search heuristics, and thus mechanisms that adapt them can be beneficial to further guide the algorithm to better solutions. Third, we limit the computational time of the matheuristics and use a heuristic for the warm-start. The interaction of these two methods and the solver has a larger effect on some problems than others. We observed that for some instance categories, the solver was able to find improvements at every stage, but in some others failed to find a different solution than the heuristic within the allowed time. These limitations in our study, however, give rise to new research questions, specially in the combination of exact and heuristic approaches, i.e., matheuristics: how to tune the parameters, how to adapt the algorithm that uses the parameters after each iteration, and how to handle the interaction between solver and solution are examples of promising research lines.

Even though we analyze one trigger for re-scheduling, i.e., fixed intervals, it is important to note there can be other triggers such as change in the status of trucks, traffic data, or cancellation of customer requests $[42,106]$. Different triggers for re-scheduling may require different FCs. For example, fixing routes with the shortest traveling time from the previous schedule may not be the best option if the trigger for re-scheduling was a delay due to increased traffic. Furthermore, the interaction between the real-time aspect of synchromodality and the re-scheduling trigger needs to be considered. When the time required for re-scheduling (e.g, computation time of the matheuristic) is larger than the effect of a trigger (e.g., delay), it can be that re-scheduling is even not necessary. Overall, these re-scheduling aspects in 
synchromodality need to be investigated and tackled within a dynamic solution approach.

\subsection{Conclusions}

We developed a MILP and a matheuristic to schedule drayage operations in synchromodal transport. Timing, routing, and long-haul terminal assignment decisions are integrated and simultaneously considered. Dynamic scheduling is done as new information is revealed throughout the day.

Through numerical experiments, we studied the performance of our adaptations to the MILP model and fixing criteria in the matheuristic. Overall, we observed that the gains of our approach are dependent on problem attributes such as customer dispersion, time-window lengths, and dynamic re-scheduling. Further research on the relation between these characteristics and our matheuristics is needed. The proper handling of such relations is essential for scheduling drayage operations in synchromodal transport.

\section{Appendix}

\section{5-A Small Example of the Model}

In this appendix, we illustrate the two directed graphs for the model in Section 5.4, and illustrate the workings of the valid inequalities therein. The two directed graphs are exemplified in Figures 5.5 and 5.6. To simplify the description of these graphs, we have given nodes different identifiers. However, the identifiers in 5.6 are the ones used for defining $\mathcal{V}$ the model.

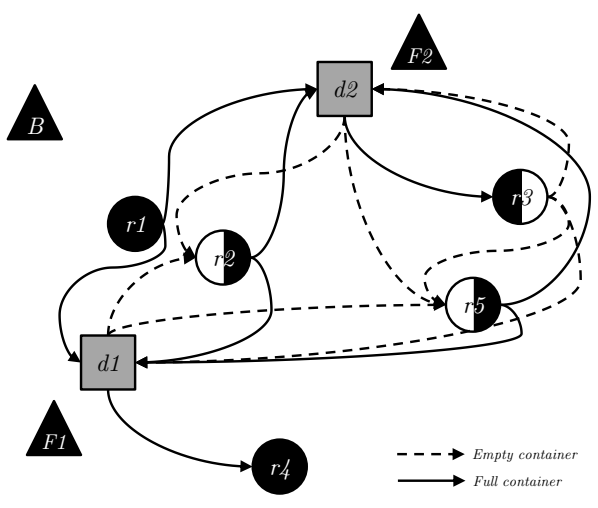

Figure 5.5: Example $\mathcal{G}=(\mathcal{V}, \mathcal{A})$

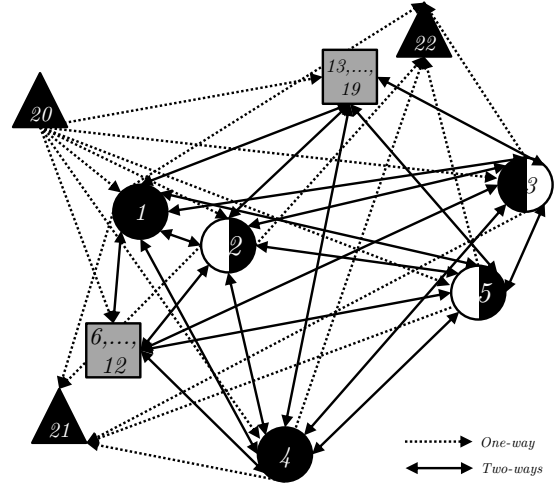

Figure 5.6: Example $\mathcal{G}^{\prime}=\left(\mathcal{V}, \mathcal{A}^{\prime}\right)$ 
In Figure 5.5, various drayage requests and their job-arcs $\mathcal{A}$ are shown. Nodes $r 1$ through $r 5$ represent drayage requests. Node $r 1$ has a full container that needs to be transported to any terminal (i.e., job type 11). Node $r 2$ requires the delivery of an empty container, and subsequently its transport to any terminal after it is loaded (i.e., job type 7). Note that this empty container is a special resource found only in the terminals, but not in any other customer. Node $r 3$ needs the delivery of a full container from a fixed terminal, and subsequently returns the empty container after unloading to either a terminal or a customer requiring such a container (i.e., job configuration 2). Node $r_{4}$ needs a full container to be delivered (i.e., job type 3). Node $r 5$ needs the delivery of an empty container delivered (of the same type as the one from Node r3) and subsequently its transport to any terminal after it is loaded. Nodes $d 1$ and $d 2$ represent two terminals. Node $B$ represents the beginning location of trucks and Nodes F1 and F2 the end-locations of trucks, which serve for routing purposes only and thus have no job-arcs attached to them.

In Figure 5.6, nodes $\mathcal{V}$ with their actual indices and routing-arcs $\mathcal{A}^{\prime}$ are shown. Nodes 1 through 5 represent drayage requests. Nodes 6 through 12 represent the first terminal and Nodes 13 through 19 represent the second one. The reason why nodes are duplicated $N_{d}=7$ times has to do with the number of times a truck most depart or arrive due to the potential execution of requests (i.e., number of job-arcs in Figure 5.5 connected to a terminal). Note that between all job and terminal nodes, there are two-way arcs (i.e., incoming and outgoing directions). Nodes 20 through 21 represent the begin and end-locations of trucks. Since trucks can either depart or arrive from these nodes, but not both, they only have one-way arcs.

In this example, the valid inequalities (5.3) and (5.4) work as follows. In the first valid inequality, $M^{\mathrm{DE}}$ is the maximum number of routing-arcs between all terminal nodes that can be traversed. Remind that, in the routing graph, there are a total of 14 nodes representing the terminals, i.e. Nodes 6 through 19. Now, a vehicle would visit two terminal nodes consecutively (i.e., traverse a routing-arc between two terminal nodes) only if it delivers a container to a terminal and subsequently picks up another container at the same or another terminal. In this example, there are at most 4 containers delivered to a terminal, i.e., loaded containers of $r 1, r 2$, and $r 5$, as well as the empty container of $r 3$. Furthermore, there are at most 3 containers picked up from a terminal, i.e., empty containers for $r 2$, and $r 5$, as well as the loaded container for $r 3$ (in case of terminal d2) and for $r_{4}$ (in case of terminal $d 1$ ). If they all would be delivered and picked up at a terminal, then 3 routing-arcs between two terminal nodes would need to be traversed, i.e. $M^{\mathrm{DE}}=3$. In the second valid inequality, $M_{d}^{\mathrm{DI}}$ is the maximum number of $\operatorname{arcs}(i, j) \in \mathcal{A}^{\prime}$ that can be traversed between the replicated nodes of depot $d \in \mathcal{D}$, i.e. $i, j \in \mathcal{U}_{d}$. The logic is similar to the one of the first valid inequality, but now more specific to a terminal (i.e., Nodes 6 through 12 and Nodes 13 through 19). Due to requests $r 3$ and $r 4$ having part of their job fixed to one terminal, then $M_{1}^{\mathrm{DI}}=M_{2}^{\mathrm{DI}}=2$.

To illustrate the performance improvements attained by the valid inequalities, consider the following instance parameters. Nodes are located in a plane of $15 \times 13$ distance units from the lowest leftmost node. One distance unit is equivalent to one minute of traveling. Time-windows of customers have an average of 341 minutes, and are dispersed throughout the working day. There are two vehicles available. The first one has a fixed cost of 1000 and can serve all customers. The second vehicle has a fixed cost of 2000 and can 


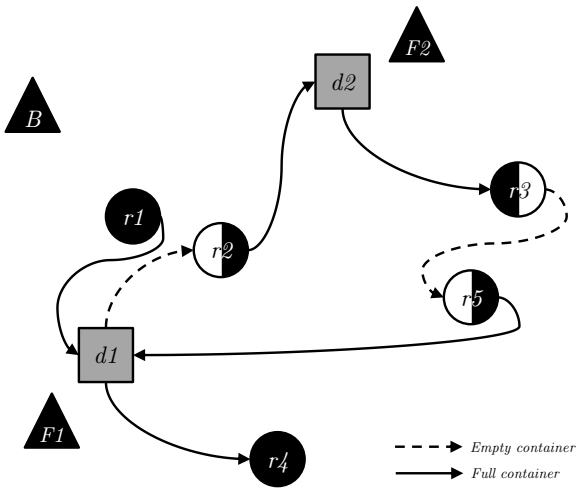

Figure 5.7: Example solution in $\mathcal{G}=(\mathcal{V}, \mathcal{A})$

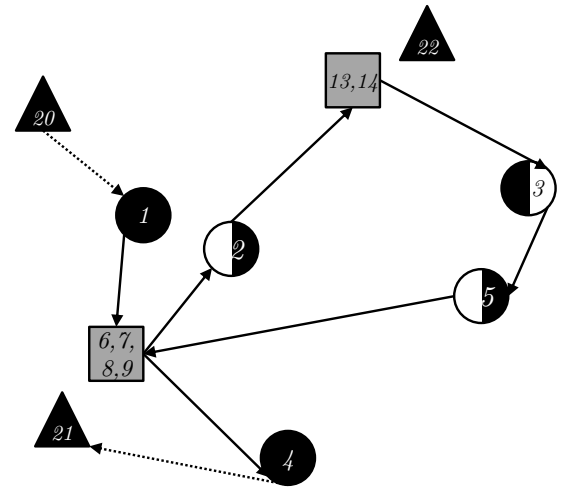

Figure 5.8: Example solution in $\mathcal{G}^{\prime}=\left(\mathcal{V}, \mathcal{A}^{\prime}\right)$

serve only customers 3 and 5 . The maximum working time of both vehicles is of 720 minutes. In Table 5.3 we see the gains in solving time due to the valid inequalities. The optimal solution for this example is to use the first vehicle only in the following route 20-1-6-7-2-13-14-3-5-8-9-4-21, as shown in Figures 5.7 and 5.8. We also considered a more disperse distribution of the locations 150x130, with two vehicles of each type, and all other parameters remaining the same. The solution uses only two vehicles, the first one following the route 20-1-6-7-2-8-9-4-21 and the second one the route 20-13-3-5-10-21.

Table 5.3: Average performance of the Valid Inequalities (VI) in the numerical example, over 10 replications

\begin{tabular}{lcccccc}
\hline \multirow{2}{*}{ Method } & \multicolumn{3}{c}{ Normal } & \multicolumn{3}{c}{ Dispersed } \\
& Costs & Time (s) & Gap & Costs & Time (s) & Gap \\
\hline MILP & 1051.70 & 1.03 & 0.00 & 2622.31 & 6.27 & 0.00 \\
VI (5.3) & 1051.70 & 0.68 & 0.00 & 2622.31 & 2.28 & 0.00 \\
VI (5.4) & 1051.70 & 0.73 & 0.00 & 2622.31 & 3.33 & 0.00 \\
VI (5.3) and (5.4) & 1051.70 & 0.64 & 0.00 & 2622.31 & 2.19 & 0.00 \\
\hline
\end{tabular}




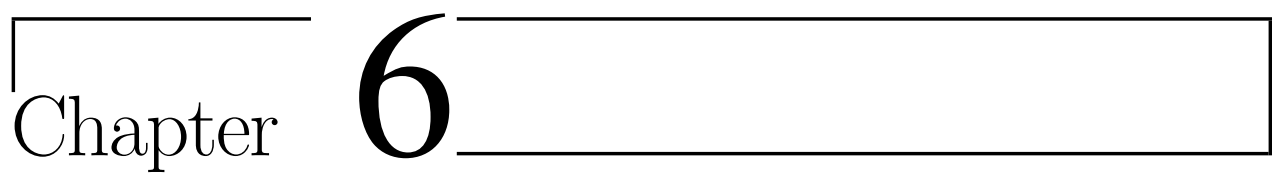

\section{Integrated Long-haul and Drayage Transport}

In this chapter, we study the problem of the integrated scheduling of drayage operations and long-haul transport. Although different in time span and characteristics of execution, these two processes have an impact on each other and their interaction has a direct influence on the overall performance of the transport network over time. We propose a simulation based integration of the Mixed-Integer Linear Programming (MILP) model for the drayage operations and the Markov Decision Process (MDP) model for the long-haul transport. We analyze the interfaces between these models, outline the challenges of integrating them, and design a heuristic approach to the simulation based integration. In a series of numerical experiments, we study the calibration of these components and the heuristic, and evaluate the cost savings compared to a non-integrated approach, using various transport network configurations. We discuss limitations of our model and experiments, and provide guidelines for further research for the integrated scheduling of drayage and long-haul operations in synchromodal transport. This chapter is based on our publication in [74].

\subsection{Introduction}

In previous chapters, we have separately studied different parts of a synchromodal transport network. This separation allowed us to isolate some of the characteristics of synchromodal transport that affect a part of transport directly, such as transfers in long-haul transport and initial terminal assignment to pre-haulage jobs in drayage operations. However, and as motivated in Chapter 1, network-wide and anticipatory decisions in synchromodal transport are necessary to improve the overall efficiency and sustainability of freight transport. In this chapter, we study the integrated scheduling of drayage operations and long-haul transport. 
The focus of this chapter is on Part 4 of the division of the synchromodal transport network presented in Figure 1.6 of Chapter 1. We combine the problem characteristics of Chapters 4 and 5 and study the integration of their scheduling methods.

Drayage operations and long-haul transport have usually been scheduled independently due to their difference in time span of execution. On the one hand, drayage operations are carried out by trucks in small geographical areas where each truck can move more than one freight per day. On the other hand, long-haul transport is carried out by high-capacity modes such as trains and barges, over long geographical areas with traveling times typically lasting more than a day. Yet, schedules of the drayage operations can influence the long-haul transport, and the long-haul schedules can influence the opportunities for the drayage operations in synchromodal transport. To exemplify this two-sided influence, consider an LSP deciding on which of two terminals to bring a freight to, for the start of its long-haul. If the focus is on drayage performance, the terminal closest to the origin of the freight is most likely to be chosen during the scheduling of drayage operations, even if the furthest terminal has better consolidation opportunities for long-haul transport. If the focus is on the long-haul, the terminal with the best consolidation opportunities is most likely to be chosen, even at the expense of unnecessary drayage operations costs. Traditionally, literature on multi-modal long-haul transport has not considered drayage operations as a whole (e.g,. movement of empty containers, pre- and end-haulage freight, etc.), even though the integration of independent scheduling activities using up-to-date network information has been identified as fundamental to improve the performance of multi-modal transport [17].

Integrating the scheduling of drayage operations and long-haul transport with the goal of optimizing performance over the entire network and over time is difficult for two reasons. First, freights that arrive each day, and their characteristics, are uncertain. Even though the uncertainty of freight arrivals is faced first by the drayage operations, the long-haul transport also faces uncertainty determined by the arrival of freights to the system and the drayage decisions (e.g., for the same drayage request, different terminals may be more suitable on different days). Second, the individual drayage and long-haul scheduling decisions have an impact on each other's feasible decision space. A focus on network-wide performance and interaction between individual scheduling decisions may signify the deterioration of one of the individual performances (e.g., bringing a freight to a further terminal may be beneficial for the long-haul and the network as a whole but detrimental for the drayage). Balancing such a trade-off requires a careful analysis of the problem characteristics. Considering these challenges, the objective of this chapter is two-fold: (i) to design an approach that integrates the scheduling decisions of drayage operations and long-haul transport, as well as their network-wide performance, considering stochastic freight arrivals and (ii) to provide insights into the use of our approach under various freight demand patterns and performance settings.

Following our objective, the contribution of this chapter is two-fold. First, we design a simulation-based approach to overcome the optimization challenges of integrating the scheduling of drayage operations and long-haul transport. Our approach iteratively solves the MixedInteger Linear Programming (MILP) model of the drayage operations from Chapter 5 and the Markov Decision Process (MDP) model of the long-haul transport from Chapter 4. The MILP considers long-haul performance based on the solution of the MDP while the MDP uses 
freight arrival rates based on the solution of the MILP. To use our approach on large instances, we use a Math-Heuristic (MH) for solving the MILP, as we also did in Chapter 5, and an Approximate Dynamic Programming (ADP) algorithm for solving the MDP, as we also did in Chapters 3 and 4. Second, we study the use of our approach under diverse instances of freight demand and drayage/long-haul costs. In each instance of our numerical study, we evaluate the relation between the decisions and costs of the drayage operations and the long-haul transport. In addition, we evaluate the gains of integrated scheduling compared to a benchmark heuristic. Based on the evaluations, we gather managerial insights for integrated scheduling of drayage operations and long-haul transport and provide guidelines for further research.

The remainder of this chapter is structured as follows. In Section 6.2, we examine literature related to drayage scheduling, long-haul scheduling, and their integration. In Section 6.3, we describe and formulate the problem, along with the MILP and MDP models for the drayage and long-haul scheduling, respectively. In Section 6.4, we present our simulation-based approach and describe the MH and ADP heuristics. In Section 6.5, we present the results of our numerical experiments. In Section 6.6, we discuss the research and managerial implications of our numerical experiments and scheduling approach. Finally, we present our conclusions in Section 6.7.

\subsection{Literature Review}

In this section, we briefly review the literature on scheduling drayage operations as well as scheduling long-haul transport in a multi-modal transport network. Since we are interested in the integration of decisions and performance of these two parts of the network for synchromodal transport, we focus on the relations between drayage operations and long-haul transport present in the individual studies about scheduling each part of transport. For a more in-depth discussion of models and solution methodologies for scheduling drayage operations and long-haul transport, we refer the reader to our previous chapters. We start by discussing the relation of studies about drayage operations with long-haul transport. Afterwards, we discuss the relation of studies about long-haul transport with drayage operations. We finalize by stating our scientific contribution to integrated scheduling in synchromodal transport.

The impact of drayage operations on the total costs of multi-modal transport is large [48], but, research on their scheduling has been limited [13]. Particularly, studies on the scheduling of drayage operations considering multiple characteristics of synchromodal transport, such as the flexible choice of pre-haulage terminal (i.e., start terminal for the long-haul) and the use of network information (e.g., including long-haul performance), are limited, as we discussed in the literature review of Chapter 5. Moreover, most studies about scheduling drayage operations that include some form of network information usually focus on special cases of information that impact decisions rather than network-wide performance. For example, Taylor et al. [98] include the flexible selection of an initial ramp (i.e., terminal) for freight in truck-rail transport with the objective of minimizing trucking costs. Marković, Drobnjak, and Schonfeld [57] consider stochastic delays at intermodal terminals that impact the drayage costs. Studies that explicitly include performance on both drayage and long-haul do so for special applications and objectives. For example, Verma et al. [111, 112] develop a 
drayage model considering total costs, that includes long-haul costs, for a truck-rail network of hazardous materials and with an additional objective and set of constraints regarding the risk of transporting the hazardous materials. To summarize, scheduling of drayage operations have mostly been studied considering only a few forms of flexibility and without considering dynamic long-haul performance.

Compared to drayage operations, scheduling long-haul transport in multi-modal transport has been widely studied, as we have discussed in Chapters 2 and 4. Studies that are closely related to synchromodal transport are those belonging to the Dynamic Service Network Design (DSND) class. DSND studies have built a strong body of knowledge for modeling the evolution of network-wide decisions and performance through time [115] even though their complexity has made it difficult to include stochastic components [92]. Furthermore, most DSND studies, independent of whether they include stochastic components, do not explicitly model characteristics of the drayage operations. Some of the studies, such as [21], point out that costs from the origin of freights to the initial terminals can be calibrated to mimic drayage operations. Yet, these costs might depend on the status of the network, as we exemplified in Section 6.1. To summarize, scheduling long-haul transport in synchromodality can build upon multi-modal studies with stochastic demand and extend these studies by including the richness of drayage operations.

To conclude, most literature about scheduling drayage operations and scheduling long-haul transport have largely ignored the impact of both decision processes on each other's operations. Furthermore, some of the key characteristics of synchromodal transport networks have not been included in the modeling assumptions. Considering this, the contribution of our chapter to the scientific literature is two-fold. First, we design a scheduling approach that focuses on the interaction between drayage and long-haul decisions and performance, considering multiple characteristics of synchromodal transport such as stochastic freight arrivals and flexible terminal assignment. Second, we provide insight into how the characteristics of a synchromodal network influence the integration of drayage and long-haul scheduling decisions.

\subsection{Problem Description and Formulation}

We study the problem of scheduling drayage operations and long-haul transport in a synchromodal network, over a multi-period horizon $\mathcal{T}$, with the objective of minimizing the total expected costs over the network and over the horizon. Without loss of generality, we assume that in each period $t \in \mathcal{T}$, one long-haul mode departs; and that in between two consecutive periods (i.e., two consecutive long-haul mode departures), drayage operations are carried out. We consider stochastic arrival of freight for the drayage, and consequently, stochastic and drayage-dependent arrival of freight for the long-haul. We illustrate the timing of the freight arrivals, transport processes and scheduling decisions using Figure 6.1. Consider, for example, one terminal with a long-haul mode departing daily at noon and freights that arrive throughout the day. The long-haul schedule $\left(x_{t}^{\mathrm{L}}\right)$ for day $t$ is created at noon, just before the mode departs. The drayage schedule $\left(x_{t}^{\mathrm{D}}\right)$ is created at midnight (i.e., the beginning of day $t$ ), considering freights that arrived before midnight $\mathcal{F}_{t}$ as well as routing costs and terminal costs $\left(C_{t}^{\mathrm{L}}\right)$. Drayage operations are completely carried out between 
midnight and noon of day $t$. Freights $\left(f_{t}^{\mathrm{L}}\right)$ to be considered for the schedule of the long-haul at day $t\left(x_{t}^{\mathrm{L}}\right)$ depend then on freights that were brought by the drayage operations of that day $\left(x_{t}^{\mathrm{D}}\right)$ in addition to the freights that were not scheduled for the long-haul the day before $\left(x_{t-1}^{\mathrm{L}}\right)$. This example shows our modeling assumption of alternating one long-haul schedule creation and one drayage schedule creation. We note, however, that this assumption can easily be generalized to a different timing of schedule creation for the two parts of transport, as long as (i) the arrival of freight to the each long-haul scheduling decision is dependent on all drayage scheduling decisions performed in between two consecutive long-haul scheduling decisions, and (ii) each time a drayage schedule is created, the impact on the next long-haul schedule is considered, even if the next long-haul scheduling decision occurs after more drayage scheduling decisions. Note that the superscript ' $D$ ' denotes the parameters related to the drayage scheduling and ' $\mathrm{L}$ ' the parameters related to the long-haul scheduling.

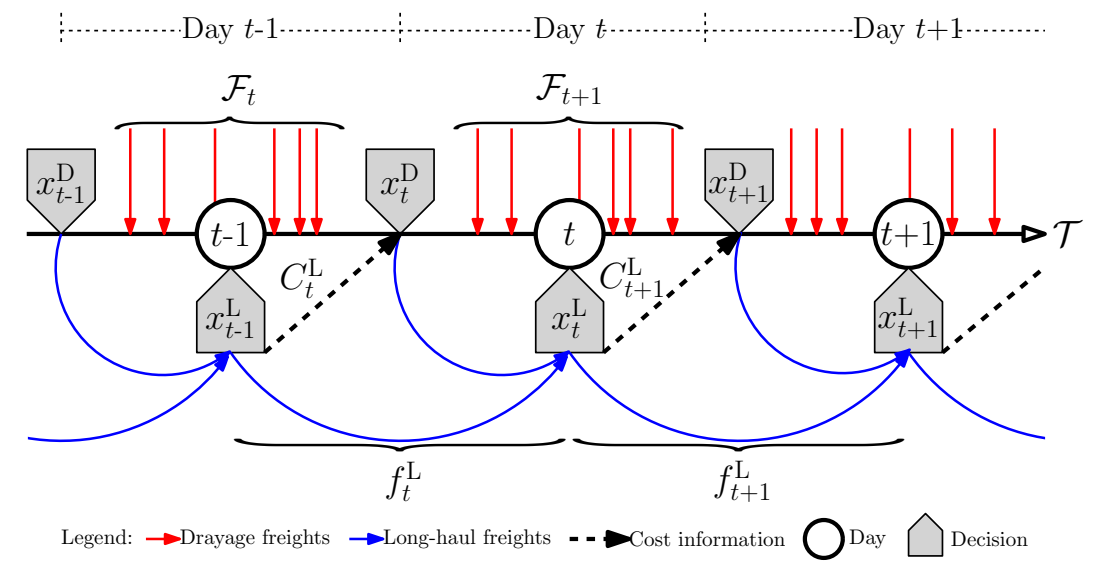

Figure 6.1: Timing of freight arrivals, transport processes, and scheduling decisions

In each period $t \in \mathcal{T}$, the planner must first schedule the drayage operations and then schedule the long-haul transport, as exemplified in Figure 6.1. Drayage scheduling includes two decisions: (i) the initial terminal where each pre-haulage freight will begin its long-haul and (ii) the route of trucks that execute the drayage operations. Long-haul scheduling includes the decision which of the available freights at each terminal to consolidate in the long-haul modes (e.g., barge, train, etc.). Although drayage and long-haul scheduling are done separately, their decisions have an influence on the decision space of each other, and consequently, on the attainable performance over the network and over time. For instance, the assigned initial terminal of pre-haulage freights influences the route of trucks in the drayage operations and the consolidation possibilities in the long-haul modes. Before modeling these two scheduling parts and the influence they have on each other, we introduce the notation related to freight demand and transport supply in the network. For simplicity, we refer to a period $t \in \mathcal{T}$ as a day in the remainder of this chapter, noticing nevertheless that time can be discretized in 
any arbitrary interval as long as the modeling considerations mentioned before are applied.

Each day $t$, new freights $\mathcal{F}_{t}$ arrive into the network. Each freight $f^{\mathrm{N}} \in \mathcal{F}_{t}$ has a drayage request type $r^{\mathrm{D}} \in \mathcal{R}$, and depending on the request type, it has drayage destination $d^{\mathrm{D}} \in \mathcal{D}^{\mathrm{D}}$ (in case of an end-haulage job), or drayage origin $o^{\mathrm{D}} \in \mathcal{O}$ and long-haul destination $d^{\mathrm{L}} \in \mathcal{D}^{\mathrm{L}}$ (in case of a pre-haulage job). The sets of drayage destinations $\mathcal{D}^{\mathrm{D}}$, origins $\mathcal{O}$, and long-haul destinations $\mathcal{D}^{\mathrm{L}}$ denote locations in the network, as seen in Figure 6.2. The set of drayage request types $\mathcal{R}$ represents all possible drayage jobs, which includes freights that are to begin their long-haul, i.e., pre-haulage jobs, and freights that finished their long-haul and are destined to a customer, i.e., end-haulage job. Examples of drayage job types are the pick-up of a full container, the delivery of an empty container and the subsequent pick-up of the full container, delivery of a full container, etc. We consider that only pre-haulage freights use the long-haul modes and hence have a destination in the long-haul part of the network. End-haulage freights only use the trucks of the drayage part, and hence have a drayage destination but no long-haul destination. These two considerations make the network asymmetric in paths followed by pre- and end-haulage freights, as exemplified in Figure 6.2.

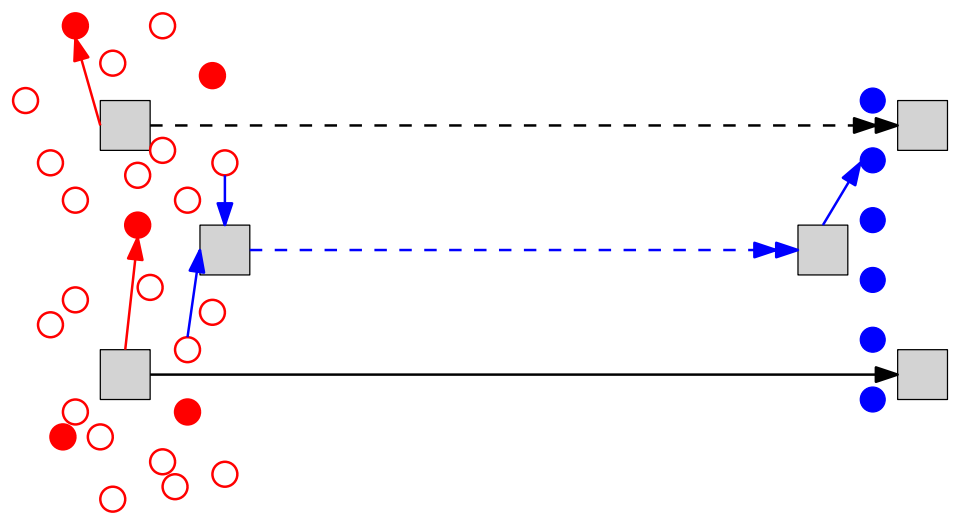

Legend:

$\bigcirc$ Drayage origins $\mathcal{O} \bigcirc$ Drayage destinations $\mathcal{D}^{\mathrm{D}} \bigcirc$ Long-haul destinations $\mathcal{D}^{\mathrm{L}} \square$ Terminals $\mathcal{H}$

$\longrightarrow$ Train $\longrightarrow$ Barge $\longrightarrow$ Path end-haulage freight $\longrightarrow$ Path pre-haulage freight

Figure 6.2: Example of network locations and transport paths of different freight.

In addition to location information, each freight $f^{\mathrm{N}} \in \mathcal{F}_{t}$ has drayage time-window $\left[e^{\mathrm{D}}, l^{\mathrm{D}}\right] \in \mathcal{T}^{\mathrm{D}}$, and long-haul time-window length $l^{\mathrm{L}} \in \mathcal{T}^{\mathrm{L}}$. The set of drayage times $\mathcal{T}^{\mathrm{D}} \subset \mathbb{R}$ represents the time during a single day when trucks are working. The drayage time-window $\left[e^{\mathrm{D}}, l^{\mathrm{D}}\right] \in \mathcal{T}^{\mathrm{D}}$ defines the earliest and latest arrival time of a truck to the drayage origin. The long-haul time-window length $l^{\mathrm{L}} \in \mathcal{T}^{\mathrm{L}}$ represents the number of days, relative to the current day $t$, in which a freight must be at its final long-haul destination. In other words, a freight with long-haul time-window length $l^{\mathrm{L}}$ at day $t$ is due on $\left(t+l^{\mathrm{L}}\right)$. Using the notation 
just introduced, we can describe a freight $f^{\mathrm{N}} \in \mathcal{F}_{t}$ with the tuple in (6.1).

$$
f^{\mathrm{N}}=\left(r^{\mathrm{D}}, d^{\mathrm{D}}, o^{\mathrm{D}}, e^{\mathrm{D}}, l^{\mathrm{D}}, d^{\mathrm{L}}, l^{\mathrm{L}}\right)
$$

Although freights are not known before they arrive, we assume there is probabilistic knowledge about their arrival intensity and about their characteristics. Between two consecutive days, $f \in \mathbb{N}$ freights arrive with probability $p_{f}^{\mathrm{F}}$. Each freight has drayage request type $r^{\mathrm{D}} \in \mathcal{R}$ with probability $p_{r}^{\mathrm{R}}$ and drayage origin $o^{\mathrm{D}} \in \mathcal{O}$ with probability $p_{o}^{\mathrm{O}}$. Depending on the request type $r^{\mathrm{D}} \in \mathcal{R}$, a freight has drayage destination $d^{\mathrm{D}} \in \mathcal{D}^{\mathrm{D}}$ with probability $p_{r, d^{\mathrm{D}}}^{\mathrm{DD}}$, longhaul destination $d^{\mathrm{L}} \in \mathcal{D}^{\mathrm{L}}$ with probability $p_{r, d^{\mathrm{L}}}^{\mathrm{DL}}$, earliest drayage truck arrival $e^{\mathrm{D}} \in \mathcal{T}^{\mathrm{D}}$ with probability $p_{r, e^{\mathrm{D}}}^{\mathrm{ETD}}$, latest drayage truck arrival $l^{\mathrm{D}} \in \mathcal{T}^{\mathrm{D}}$ with probability $p_{r, l^{\mathrm{D}}}^{\mathrm{LTD}}$, and long-haul time-window length $l^{\mathrm{L}} \in \mathcal{T}$ with probability $p_{r, l \mathrm{~L}}^{\mathrm{LTL}}$. To be concise, we enclose all the probabilities mentioned before, which describe all freights that arrive to the network, in the set $\mathcal{P}^{\mathrm{D}}$.

To execute all drayage operations, there is a fleet $\mathcal{K}$ of heterogeneous trucks. The subset of trucks that can carry out drayage request type $r^{\mathrm{D}} \in \mathcal{R}$ is denoted by $\mathcal{K}_{r}$. Note that drayage requests not only differ in whether they are a pre- or end-haulage job, or whether they use an empty or full container, but also on required driver clearance, truck chassis, etc. The time to execute a drayage request type $r^{\mathrm{D}} \in \mathcal{R}$, e.g., load/unload a container, by truck $k \in \mathcal{K}$ is denoted by $T_{r, k}^{\mathrm{SD}}$. Truck $k \in \mathcal{K}$ must begin its route in terminal $B_{k}$ and finalize it in terminal $F_{k} \in \mathcal{H}$, where $\mathcal{H}$ represents all terminals available in the transport network. Each terminal $h \in \mathcal{H}$ has a service time $T_{h}^{\mathrm{SH}}$, which denotes the time to serve a drayage truck. To represent all locations in the drayage, i.e., origins, drayage destinations, and terminals, we define the set $\mathcal{N}^{\mathrm{D}}=\mathcal{O} \cup \mathcal{D}^{\mathrm{D}} \cup \mathcal{H}$ and index it with $i$ and $j$. We denote the traveling time between locations $i$ and $j$ in $\mathcal{N}^{\mathrm{D}}$ with $T_{i, j}^{\mathrm{TD}}$. The routing costs of truck $k \in \mathcal{K}$ consist of a fixed cost $C_{k}^{\mathrm{FD}}$ for its use and a variable cost $C_{k, i, j}^{\mathrm{VD}}$ for traveling between locations $i$ and $j$ in $\mathcal{N}^{\mathrm{D}}$.

To execute the long-haul transport, there are various high-capacity modes traveling between locations in the long-haul, i.e., long-haul terminals and long-haul destinations. We denote all locations in the long-haul with $\mathcal{N}^{\mathrm{L}}=\mathcal{H} \cup \mathcal{D}^{\mathrm{L}}$ and index it with $i$ and $j$. We define a service as a specific long-haul transport mode going from location $i$ to $j$, both in $\mathcal{N}^{\mathrm{L}}$, with a capacity of $Q_{i, j}$ freights and a duration of $T_{i, j}^{\mathrm{TL}}$, which includes traveling and handling times at the locations. Similar to the trucks, we consider the long-haul costs of the service between $i$ and $j$ to consist of a fixed component $C_{i, j}^{\mathrm{FL}}$ if at least one freight uses this service, and a variable cost $C_{i, j, d}^{\mathrm{VL}}$ per freight with destination $d \in \mathcal{D}^{\mathrm{L}}$ consolidated in this service. Furthermore, we consider an additional long-haul cost $C_{i, j, \mathcal{G}}^{\mathrm{GL}}$ per group of long-haul destinations $\mathcal{G} \subseteq \mathcal{D}^{\mathrm{L}}$ visited after, or during, the service between $i$ and $j$. This cost component can be used to capture, for example, last-mile routing costs after the service between two intermodal terminals, or stopping costs at intermediate destinations visited by the service.

Before formulating our integrated model of drayage and long-haul operations, we summarize the four main assumptions we make in the list below. Assumptions 1 and 2 have already been discussed. Assumptions 3 and 4 are discussed in subsequent sections, where we indicate whether they can easily be generalized or require additional work. 
1. In each period, one long-haul mode departs and one long-haul transport schedule is made.

2. In between two consecutive periods, drayage operations are carried out and one drayage schedule is made.

3. All pre-haulage freights that arrive are "released" for long-haul transport, i.e., can be immediately transported in the long-haul after drayage.

4. Long-haul services that do not go to final destinations of freights, i.e., services between intermediate terminals, have a duration of at most one day.

The remainder of this section is divided into three parts. In Section 6.3.1, we present the model for scheduling drayage operations. In Section 6.3.2, we present the model for scheduling long-haul transport. In Section 6.3.3, we formulate the integration mechanisms of these two models. In contrast to previous chapters, some of the notation in this chapter is in lowercase instead of uppercase, or uses superscript. Lowercase notation represents decision variables in the integrated model. The superscripts distinguish whether variables and parameters are part of the drayage or the long-haul scheduling.

\subsubsection{Drayage Scheduling}

Our model for drayage scheduling is based upon the Mixed-Integer Linear Programming (MILP) model from Chapter 5. We use the two graphs $\mathcal{G}$ and $\mathcal{G}^{\prime}$ defined in Section 5.4 as follows. Nodes $\mathcal{V}$ are defined based on the set $\mathcal{N}^{\mathrm{D}}$ and freights $F \in \mathcal{F}_{t}$ in this chapter. Remind that each freight $F \in \mathcal{F}_{t}$ has a drayage request type, and based on this, it may have a drayage origin, drayage destination, and long-haul destination. $\operatorname{Arcs} \mathcal{A}$ and $\mathcal{A}^{\prime}$ are built according to the feasibility constraints and routing connections dictated by the drayage request type $r^{\mathrm{D}}$ of each freight $F \in \mathcal{F}_{t}$, respectively. To ease the presentation, we describe in the following paragraphs the modifications to the objective function and decision variables necessary to use the MILP model from Section 5.4. All other constraints and valid inequalities of the MILP can directly be used.

We model the drayage decisions using the binary variable $x_{k, i, j, t}^{\mathrm{D}}$, which gets a value of 1 if truck $k \in \mathcal{K}$ moves from location $i \in \mathcal{N}^{\mathrm{D}}$ to location $j \in \mathcal{N}^{\mathrm{D}}$ at time $t$ and 0 otherwise. Using this variable, we can define the drayage costs $z_{t}^{\mathrm{D}}$ at day $t$ as shown in (6.2). This cost definition has been used in traditional drayage models such as the one from [12]. However, this objective does not include a terminal assignment costs for pre-haulage jobs, which is necessary to integrate the long-haul transport process to drayage decisions.

$$
z_{t}^{\mathrm{D}}=\sum_{k \in \mathcal{K}}\left(C_{k}^{\mathrm{FD}} \cdot \sum_{j \in \mathcal{N}^{\mathrm{D}}} x_{k, B_{k}, j, t}^{\mathrm{D}}\right)+\sum_{k \in \mathcal{K}} \sum_{i \in \mathcal{N}^{\mathrm{D}}} \sum_{j \in \mathcal{N}^{\mathrm{D}}} C_{k, i, j}^{\mathrm{VD}} \cdot x_{k, i, j, t}^{\mathrm{D}}
$$

In the MILP model from Chapter 5 , we had a cost $C_{i, j, t}^{\mathrm{AD}}$ for assigning a freight with origin $i \in \mathcal{O}$ to terminal $j \in \mathcal{H}$ is added, as shown in (6.3). This cost term can be used to capture the long-haul transport process, for example, by scaling it proportionally to the average long-haul cost over all modes departing from each terminal $j \in \mathcal{H}$. However, this 
static cost term does not necessarily capture the consolidation opportunities throughout the network and throughout the time horizon that were mentioned in Section 6.1. Examples of such opportunities include economies of scale (e.g., reduction of cost per freight with an increasing number of freights brought to a terminal) and the combination of freights with similar destinations (e.g., there are freights present at a terminal that go to the same area as the long-haul destinations of new drayage freights).

$$
\tilde{z}_{t}^{\mathrm{D}}=z_{t}^{\mathrm{D}}+\sum_{k \in \mathcal{K}} \sum_{i \in \mathcal{O}} \sum_{j \in \mathcal{H}} C_{i, j, t}^{\mathrm{AD}} \cdot x_{k, i, j, t}^{\mathrm{D}}
$$

To capture the cost of the opportunities just mentioned, the static terminal assignment cost has to be changed to a function that depends on the freights that are already at the long-haul terminals, in addition to freights that are brought to those terminals by the drayage operations. We introduce the integer variable $f_{j, d, l, t}^{\mathrm{L}}$, which counts the number of freights at terminal $j \in \mathcal{H}$ at time $t$ that have long-haul destination $d \in \mathcal{D}^{\mathrm{L}}$ and long-haul time-window length $l \in \mathcal{T}$. These freights are already at the terminals, meaning that they are not considered for the drayage decision but for the long-haul decision. For simplicity, we define the vectors $f_{t}^{\mathrm{L}}$ and $x_{t}^{\mathrm{D}}$ as shown in (6.4) and (6.5), respectively. Using these vectors, we introduce the function $C_{t}^{\mathrm{L}}\left(f_{t}^{\mathrm{L}}, x_{t}^{\mathrm{D}}\right)$ and re-define the drayage cost $\tilde{z}_{t}^{\mathrm{D}}$ considering the longhaul as shown in (6.6) instead of (6.3). Remind that the function $C_{t}^{\mathrm{L}}$ has cost information resulting from the previous long-haul schedule (as shown in Figure 6.1) that looks ahead into future long-haul performance. This modification is key to integrate the long-haul transport performance over time into the decision related to drayage operations, as we will elaborate upon later on in Section 6.3.3. In the following, we focus on the long-haul scheduling decisions.

$$
\begin{gathered}
f_{t}^{\mathrm{L}}=\left[f_{j, d, l, t}^{\mathrm{L}}\right]_{\forall j \in \mathcal{H}, d \in \mathcal{D}^{\mathrm{L}}, l \in \mathcal{T}} \\
x_{t}^{\mathrm{D}}=\left[x_{k, i, j, t}^{\mathrm{D}}\right]_{\forall k \in \mathcal{K}, i \in \mathcal{N}^{\mathrm{D}}, j \in \mathcal{N}^{\mathrm{D}}} \\
\tilde{z}_{t}^{\mathrm{D}}=z_{t}^{\mathrm{D}}+C_{t}^{\mathrm{L}}\left(f_{t}^{\mathrm{L}}, x_{t}^{\mathrm{D}}\right)
\end{gathered}
$$

\subsubsection{Long-haul Scheduling}

In the previous section, we described how decisions in the drayage operations can look ahead into the performance of long-haul transport. In this section, we describe how the Markov Decision Process (MDP) from Chapter 4 can be used for scheduling long-haul transport considering drayage operations. For example, the resulting policy of the MDP, which has an estimated downstream (i.e., future) cost per state, can be used for defining the terminal assignment costs in the drayage assignment costs. To simplify the integration of the long-haul scheduling and the drayage scheduling in this chapter, we make three assumptions to reduce the complexity of the model from Section 4.4. First, we assume that all freights that arrive at an initial long-haul terminal are "released" for transport (i.e., can immediately be transported in the long-haul after drayage). Second, we assume that movements between initial terminals are not allowed (i.e., once the drayage brings a freight to an initial terminal, it departs from that terminal by the long-haul mode). Third long-haul services that do not go to final destinations 
of freights, i.e., services between intermediate terminals, have a duration of at most one day. These assumptions allows us to omit some time information required in the state definition of Equation (4.1). To not repeat the entire approach, we describe in the following paragraphs the modifications and changes necessary to use the MDP model in Section 4.4 in this chapter.

We model the long-haul transport decision with the integer variable $x_{i, j, d, l, t}^{\mathrm{L}}$, which counts the number of freights that will be consolidated at time $t$ in the service from $i$ to $j$ (both in $\mathcal{N}^{\mathrm{L}}$ ), which have long-haul destination $d \in \mathcal{D}^{\mathrm{L}}$, as well as long-haul time-window length $l \in \mathcal{T}$. We consider that there is only one service between locations $i$ and $j$, both in $\mathcal{N}^{\mathrm{L}}$, which can serve all destinations $d \in \mathcal{D}^{\mathrm{L}}$ by stopping at each destination (e.g., train in a corridor), or by last-mile trucking after terminal $j$. In addition to the $x_{i, j, d, l, t}^{\mathrm{L}}$ variable, we introduce the auxiliary binary variable $y_{i, j, d, t}^{\mathrm{L}}$, which gets a value of 1 if there is a freight with destination $d \in \mathcal{D}^{\mathrm{L}}$ consolidated at time $t$ in the service from $i$ to $j$, both in $\mathcal{N}^{\mathrm{L}}$, and 0 otherwise, as shown in (6.7). Using these two variables, the long-haul transport costs at time $t$ are defined as shown in (6.8).

$$
\begin{aligned}
& y_{i, j, d, t}^{\mathrm{L}}=\left\{\begin{array}{ll}
1, & \text { if } \sum_{l \in \mathcal{T}}\left(x_{i, j, d, l, t}^{\mathrm{L}}\right)>0 \\
0, & \text { otherwise }
\end{array}, \forall i \in \mathcal{N}^{\mathrm{L}}, j \in \mathcal{N}^{\mathrm{L}}, d \in \mathcal{D}^{\mathrm{L}}\right. \\
& z_{t}^{\mathrm{L}}=\sum_{i \in \mathcal{N}^{\mathrm{L}}} \sum_{j \in \mathcal{N}^{\mathrm{L}}}\left(C_{i, j}^{\mathrm{FL}} \cdot \sum_{d \in \mathcal{D}^{\mathrm{L}}} y_{i, j, d, t}^{\mathrm{L}}+\sum_{d \in \mathcal{D}^{\mathrm{L}}}\left(C_{i, j, d}^{\mathrm{VL}} \cdot \sum_{l \in \mathcal{T}} x_{i, j, d, l, t}^{\mathrm{L}}\right)\right)+ \\
& \sum_{i \in \mathcal{N}^{\mathrm{L}}} \sum_{j \in \mathcal{N}^{\mathrm{L}}} \sum_{\mathcal{G} \subseteq \mathcal{D}^{\mathrm{L}}}\left(C_{i, j, \mathcal{G}}^{\mathrm{GL}} \cdot \prod_{d \in \mathcal{G}} y_{i, j, d, t}^{\mathrm{L}} \cdot \prod_{d^{\prime} \in \mathcal{G} \backslash \mathcal{D}^{\mathrm{L}}}\left(1-y_{i, j, d^{\prime}, t}^{\mathrm{L}}\right)\right)
\end{aligned}
$$

In (6.8), the first line of costs captures the economies of scale of long-haul services (through the fixed and variable cost component) and the second line captures the combination of destinations in each service (through the group cost component), in a similar way to Equation (2.3) from Chapter 2. This cost definition is different than the MDP reward function in Equation (4.5) from Chapter 4, which does not include the group cost component and which includes a reward per freight making it a profit maximization rather than cost minimization problem. However, the decision variable for freight consolidation is equivalent. For simplicity, we group all decisions (i.e, freight consolidated in long-haul modes) in the vector $x_{t}^{\mathrm{L}}$ shown in (6.9). We refer to this vector as the decision of the MDP model, and define the long-haul costs as its function, i.e., $z_{t}^{\mathrm{L}}\left(x_{t}^{\mathrm{L}}\right)$. Naturally, only freights present at a terminal at time $t$ can be consolidated in the long-haul mode departing from that terminal that day. Freights that are, and will be, present at a terminal make up the state of the MDP model. To represent them, we use the integer variable $f_{j, d, l, t}^{\mathrm{L}}$ introduced in (6.4), which counts the number of freights at terminal $j \in \mathcal{H}$ at time $t$ that have long-haul destination $d \in \mathcal{D}^{\mathrm{L}}$, and long-haul time-window length $l \in \mathcal{T}$. The state $s_{t}^{\mathrm{L}}$ is defined as the vector of these variables, i.e., $f_{t}^{\mathrm{L}}$, as shown in (6.10).

$$
\begin{gathered}
x_{t}^{\mathrm{L}}=\left[x_{i, j, d, l, t}^{\mathrm{L}}\right]_{\forall i \in \mathcal{N}^{\mathrm{L}}, j \in \mathcal{N}^{\mathrm{L}}, d \in \mathcal{D}^{\mathrm{L}}, l \in \mathcal{T}} \\
s_{t}^{\mathrm{L}}=f_{t}^{\mathrm{L}}=\left[f_{j, d, l, t}^{\mathrm{L}}\right]_{\forall j \in \mathcal{H}, d \in \mathcal{D}^{\mathrm{L}}, l \in \mathcal{T}}
\end{gathered}
$$


The MDP state $s_{t}^{\mathrm{L}}$ contains all freights at the intermodal terminals at time $t$. These freights are the result of freights from the previous day $t-1$ that were not consolidated (i.e., difference between the previous state $s_{t-1}^{\mathrm{L}}$ and previous long-haul decision $x_{t-1}^{\mathrm{L}}$ ) and freights that arrived to the terminal in between the previous day $t-1$ and day $t$. These latter freights are the freights that were brought to the terminals by the drayage operations, which depend on the random freights that arrived to the system (as illustrated in Figure 6.1) and are known as the exogenous information of the MDP model. The exogenous information $w_{t}^{\mathrm{L}}$ captures the stochasticity of freight arrivals within the MDP model, which in turn, converts it into a policy that optimizes the expected performance as will be explained later on. It is important to note that for the MDP model, freight arrivals are uncertain for any future time period. However, upon implementation of the long-haul decision at time $t$ in an integrated approach, the freights are not uncertain anymore since the drayage scheduling has taken place and hence the freights that arrived to the terminal are known. The unknown and exogenous freights upon the long-haul decision at time $t$ are freights for future days since they depend on the random arrival of freights to the system and the future drayage decisions. We define freights in the exogenous information using the integer variable $\hat{f}_{j, d, l, t}^{\mathrm{L}}$, which counts the number of freights that arrived at terminal $j \in \mathcal{H}$ at time $t$, and that have long-haul destination $d \in \mathcal{D}^{\mathrm{L}}$ and long-haul time-window length $l \in \mathcal{T}$. The exogenous information $w_{t}^{\mathrm{L}}$ is defined as the vector of these variables, as shown in (6.11). Finally, using a transition function $S^{\mathrm{M}}$, we can define a state $s_{t}^{\mathrm{L}}$ at day $t$ as a function of the previous-day state $s_{t-1}^{\mathrm{L}}$, the previous-day decision $x_{t-1}^{\mathrm{L}}$ and that day's exogenous information $w_{t}^{\mathrm{L}}$, as shown in (6.12).

$$
\begin{gathered}
w_{t}^{\mathrm{L}}=\left[\hat{f}_{j, d, l, t}^{\mathrm{L}}\right]_{\forall j \in \mathcal{H}, d \in \mathcal{D}^{\mathrm{L}}, l \in \mathcal{T}} \\
s_{t}^{\mathrm{L}}=S^{\mathrm{M}}\left(s_{t-1}^{\mathrm{L}}, x_{t-1}^{\mathrm{L}}, w_{t}^{\mathrm{L}}\right)
\end{gathered}
$$

All constraints and equations from Chapter 4 (Section 4.4) that define the decision space $\mathcal{X}_{t}^{\mathrm{L}}$, state space $\mathcal{S}$, and transition function $S^{\mathrm{M}}$ are applicable to the decision, state, and exogenous information defined in this chapter, i.e., (6.9), (6.10), and (6.11), respectively. However, there are two significant modifications. First, we modify the profit maximization goal to a cost minimization objective over a multi-period horizon under stochastic, but drayage-dependent, arrivals as shown in (6.13). The objective is to find a policy $\pi \in \Pi$ (i.e., function that maps a decision to a state) that minimizes the discounted costs over the horizon, given an initial state $s_{0}^{L}$ and the exogenous information process $\left[\Omega_{t}\right]_{\forall t \in \mathcal{T}}$. Note that the exogenous information $w_{t}^{\mathrm{L}}$ is a result of the process $\Omega_{t}$, i.e., $w_{t}^{\mathrm{L}} \in \Omega_{t}$.

$$
\min _{\pi \in \Pi} \mathbb{E}\left[\sum_{t \in \mathcal{T}}\left(\cdot z_{t}^{\mathrm{L}}\left(x_{t, \pi}^{\mathrm{L}}\right)\right) \mid s_{0}^{\mathrm{L}},\left[\Omega_{t}\right]_{\forall t \in \mathcal{T}}\right]
$$

The second modification occurs in the stochastic arrival process of freights to the network considered, i.e., the exogenous information process $\left[\Omega_{t}\right]_{\forall t \in \mathcal{T}}$. In our problem, freights arrive randomly to the drayage part of the network, and subsequently, after the decisions of the drayage, to the long-haul terminals. Consequently, the arrival of freight from the exogenous in- 
formation process is stochastic and drayage-dependent. To model such an exogenous information process, we define probabilities per origin, as it is already considered in the MDP model, but we constraint each terminal to a single origin only. A single origin is used to capture the arrival rate resulting from the drayage operations to that specific terminal. In other words, the probabilities in this chapter capture the stochasticity of a certain freight type being brought to a certain terminal, opposed to the original probabilities in the MDP model in Chapter 4 (Section 4.4), which capture the stochasticity of certain freights becoming known to the system. Using our previously defined probability distributions of freight arrivals, we introduce the set of drayage-dependent probabilities $\mathcal{P}^{\mathrm{L}}$ that is used in the MDP model and encloses the following probabilities. In between two consecutive days $t-1$ and $t, f \in \mathbb{N}$ freights arrive with probability $p_{f, j, t}^{\mathrm{FL}}$ to terminal $j \in \mathcal{N}^{\mathrm{L}}$. A freight that arrives between days $t-1$ and $t$ to terminal $j \in$ $\mathcal{N}^{\mathrm{L}}$ has destination $d \in \mathcal{D}^{\mathrm{L}}$ with probability $p_{d, j, t}^{\mathrm{DL}}$, and time-window length $l \in \mathcal{T}^{\mathrm{L}}$ with probability $p_{l, j, t}^{\mathrm{LTL}}$. The probabilities in $\mathcal{P}^{\mathrm{L}}$ describe all freights that arrive to the long-haul based on the probabilities in $\mathcal{P}^{\mathrm{D}}$ and decision $x_{t}^{\mathrm{D}}$. The integration of the long-haul scheduling and the drayage scheduling is completely described by the relation between $\mathcal{P}^{\mathrm{L}}$ and decision $x_{t}^{\mathrm{D}}$.

Before analyzing the relation between the long-haul and drayage scheduling, we first take a look at the Bellman's recursion in (6.14) as a solution approach to the MDP model of the long-haul. In this recursion, the optimal expected cost $V_{t}\left(s_{t}^{\mathrm{L}}\right)$ for each state $s_{t}^{\mathrm{L}} \in \mathcal{S}$ is computed. This optimal value captures the direct cost of the long-haul $z_{t}^{\mathrm{L}}\left(x_{t}^{\mathrm{L}}\right)$ plus the expected future costs $\mathbb{E}\left[V_{t+1}\left(s_{t+1}^{\mathrm{L}}\right)\right]$. In $(6.14 \mathrm{~b})$, we use the transition function to define state $s_{t+1}^{\mathrm{L}}$ in terms of two known vectors at time $t$ : the state $s_{t}^{\mathrm{L}}$ and the decision $x_{t}^{\mathrm{L}}$; and one unknown vector at time $t$ : the exogenous information $w_{t+1}^{\mathrm{L}}$. Using $p_{\omega}^{\Omega_{t+1}}$ as the probability of exogenous information $\omega$, where $\omega$ is a realization of the exogenous information $w_{t+1}^{\mathrm{L}}$ from the set of all exogenous realizations $\Omega_{t+1}$ following from the drayage-dependent arrival distributions, we can further expand the Bellman's recursion as shown in (6.14c).

$$
\begin{aligned}
V_{t}\left(s_{t}^{\mathrm{L}}\right) & =\min _{x_{t}^{\mathrm{L}} \in \mathcal{X}_{t}^{\mathrm{L}}}\left(z_{t}^{\mathrm{L}}\left(x_{t}^{\mathrm{L}}\right)+\mathbb{E}\left[V_{t+1}\left(s_{t+1}^{\mathrm{L}}\right)\right]\right), \quad \forall s_{t}^{\mathrm{L}} \in \mathcal{S} \\
& =\min _{x_{t}^{\mathrm{L}} \in \mathcal{X}_{t}^{\mathrm{L}}}\left(z_{t}^{\mathrm{L}}\left(x_{t}^{\mathrm{L}}\right)+\mathbb{E}\left[V_{t+1}\left(S^{M}\left(s_{t}^{\mathrm{L}}, x_{t}^{\mathrm{L}}, w_{t+1}^{\mathrm{L}}\right)\right)\right]\right), \quad \forall s_{t}^{\mathrm{L}} \in \mathcal{S} \\
& =\min _{x_{t}^{\mathrm{L}} \in \mathcal{X}_{t}^{\mathrm{L}}}\left(z_{t}^{\mathrm{L}}\left(x_{t}^{\mathrm{L}}\right)+\sum_{\omega \in \Omega_{t+1}} p_{\omega}^{\Omega_{t+1}}\left(V_{t+1}\left(S^{M}\left(s_{t}^{\mathrm{L}}, x_{t}^{\mathrm{L}}, \omega\right)\right)\right)\right), \quad \forall s_{t}^{\mathrm{L}} \in \mathcal{S}
\end{aligned}
$$

For integrating the scheduling of drayage operations and long-haul transport, two components of the Bellman's recursion are important: (i) the optimal expected cost $V_{t}\left(s_{t}^{\mathrm{L}}\right)$, and (ii) the probabilities of exogenous information $p_{\omega}^{\Omega_{t+1}}$. In the following section, we discuss how these two components can be used for such an integration.

\subsubsection{Integrated Scheduling}

As mentioned earlier, we consider drayage scheduling and long-haul scheduling to be done separately, but with integrated objectives, since their decisions influence the performance of 
each other in synchromodal transport. In drayage scheduling, there is a direct integration to long-haul performance through an explicit cost component in the objective. In the long-haul scheduling, there is an indirect integration to drayage scheduling through the exogenous information. In this section, we analyze each component for integration, based on the scheduling models described in Sections 6.3.1 and 6.3.2, and describe the integrated objective function and solution approach.

In the drayage scheduling model, long-haul performance is captured through the function $C_{t}^{\mathrm{L}}\left(f_{t}^{\mathrm{L}}, x_{t}^{\mathrm{D}}\right)$ used in (6.6). To define $C_{t}^{\mathrm{L}}\left(f_{t}^{\mathrm{L}}, x_{t}^{\mathrm{D}}\right)$, we use the incremental change in the optimal expected costs $V_{t}(\cdot)$ of the Bellman's recursion in (6.14) due to a drayage decision, as shown in (6.15). This increment captures the extra costs in the long-haul when bringing drayage freight to the terminals (i.e., extra compared to not bringing any drayage freight to the terminal). These marginal costs capture opportunities that arise, for example, when a terminal already has some freight for a specific long-haul destination and it is cheap to bring pre-haulage freights that have the same long-haul destination to that terminal. To define these costs $C_{t}^{\mathrm{L}}\left(f_{t}^{\mathrm{D}}, x_{t}^{\mathrm{D}}\right)$ using $V_{t}(\cdot)$, we first look at two implications that the timing of drayage decisions (as exemplified in Figure 6.1) have. First, the freights $f_{t}^{\mathrm{L}}$ at the terminals on day $t$ form the so-called post-decision state $s_{t-1}^{\mathrm{L}, \mathrm{x}}$ of the previous day $t-1$. The post-decision state is the state of the long-haul terminals after a long-haul decision has been made but before the exogenous information arrives, or in our problem, before the drayage operations occur. The post-decision state is a function of the long-haul state and long-haul decision, as shown in (6.15c) and defined in Chapter 4 (Section 4.5). This state has an optimal expected cost of $V_{t}\left(s_{t-1}^{\mathrm{L}, \mathrm{x}}\right)$. Second, the long-haul state $s_{t}^{\mathrm{L}}$ at day $t$ and its expected downstream costs $V_{t}\left(s_{t}^{\mathrm{L}}\right)$ depend on the drayage decision $x_{t}^{\mathrm{D}}$ at day $t$ and the post-decision state $s_{t-1}^{\mathrm{L}, \mathrm{x}}$ of the previous day $t-1$. Using the transition function $S^{\mathrm{M}}$, we can define this state based on the postdecision state from the previous day $s_{t-1}^{\mathrm{L}, \mathrm{x}}$ and the drayage-dependent exogenous information $w_{t}^{\mathrm{L}, \mathrm{x}}$, as shown in (6.15b). Note that the $\emptyset$ in the $S^{\mathrm{M}}$ refers to an "empty" long-haul decision, which is used only to compute the new long-haul state after the drayage decision. Remind that $w_{t}^{\mathrm{L}, \mathrm{x}}$ is a function of the new freights $\mathcal{F}_{t}$ that arrived, randomly, for drayage and the drayage scheduling decision $x_{t}^{\mathrm{D}}$ that brought them to the terminals, as shown in $(6.15 \mathrm{~d})$.

$$
\begin{gathered}
C_{t}^{\mathrm{L}}\left(f_{t}^{\mathrm{L}}, x_{t}^{\mathrm{D}}\right)=C_{t}^{\mathrm{L}}\left(s_{t-1}^{\mathrm{L}, \mathrm{x}}, x_{t}^{\mathrm{D}}\right)=V_{t}\left(s_{t}^{\mathrm{L}}\right)-V_{t}\left(s_{t-1}^{\mathrm{L}, \mathrm{x}}\right) \\
\text { where } \\
s_{t}^{\mathrm{L}}=S^{\mathrm{M}}\left(s_{t-1}^{\mathrm{L}, \mathrm{x}}, \emptyset, w_{t}^{\mathrm{L}, \mathrm{x}}\right) \\
s_{t-1}^{\mathrm{L}, \mathrm{x}}=S^{\mathrm{M}, \mathrm{x}}\left(s_{t-1}^{\mathrm{L}}, x_{t-1}^{\mathrm{L}}\right) \\
w_{t}^{\mathrm{L}, \mathrm{x}}=S^{\mathrm{M}, \mathrm{w}}\left(\mathcal{F}_{t}, x_{t}^{\mathrm{D}}\right)
\end{gathered}
$$

In the long-haul scheduling model, drayage performance is captured indirectly through the exogenous information process $\left[\Omega_{t}\right]_{\forall t \in \mathcal{T}}$ used in the objective of the MDP in (6.13). The exogenous information $\omega \in \Omega_{t}$, and its corresponding probability $p_{\omega}^{\Omega_{t}}$, is partly due to the stochastic arrival of freights to the drayage (i.e., $\mathcal{P}^{\mathrm{D}}$ ) and the drayage decision (i.e., $x_{t}^{\mathrm{D}}$ ). 
Therefore, the solution to the Bellman equations in (6.14) is dependent on the drayage decisions. However, and as discussed in the previous paragraph, drayage decisions are dependent on the solution of the Bellman equations required for the long-haul decisions. The challenge to solve the integrated scheduling problem considering this recursion is to find a set of long-haul drayage-dependent probabilities $\mathcal{P}_{\pi}^{\mathrm{L}}$ for a policy $\pi \in \Pi$ that has two properties: (i) it results in a stable recursion, i.e., a solution to the Bellman equations that does not modify the drayage scheduling decisions that gave rise to its input long-haul drayage-dependent probabilities, and (ii) it minimizes the overall costs (i.e., drayage plus long-haul) defined in (6.16a). We now discuss the components of this objective function and the recursion therein.

$$
\begin{gathered}
\min _{\pi \in \Pi} \mathbb{E}\left[\sum_{t \in \mathcal{T}}\left(z_{t}^{\mathrm{D}}\left(x_{t, \pi}^{\mathrm{D}}\right)+z_{t}^{\mathrm{L}}\left(x_{t, \pi}^{\mathrm{L}}\right)\right) \mid s_{0}^{\mathrm{L}}, \mathcal{P}^{\mathrm{D}}, \Gamma\right] \\
x_{t, \pi}^{\mathrm{D}}=\underset{x_{t}^{\mathrm{D}} \in \mathcal{X}_{t}^{\mathrm{D}}}{\operatorname{argmin}}\left[\tilde{z}_{t, \pi}^{\mathrm{D}}\left(x_{t}^{\mathrm{D}}\right)\right] \\
\Gamma\left(\mathcal{P}^{\mathrm{D}},\left[x_{t, \pi}^{\mathrm{D}}\right]_{\forall t \in \mathcal{T}}\right)=\mathcal{P}_{\pi}^{\mathrm{L}}
\end{gathered}
$$

In the integrated objective function (6.16a), there are two new components to both the drayage and long-haul scheduling models: the drayage decisions dependent on a long-haul policy $\left(x_{t, \pi}^{\mathrm{D}}\right)$, and the function that defines the long-haul probabilities from the drayage decisions $(\Gamma)$. The drayage decision $x_{t, \pi}^{\mathrm{D}}$ at time $t \in \mathcal{T}$ given policy $\pi \in \Pi$ is defined as the decision that minimizes the drayage cost considering the long-haul $\tilde{z}_{t}^{\mathrm{D}}$, as shown in $(6.16 \mathrm{~b})$. Note that $\tilde{z}_{t}^{\mathrm{D}}$ has a subscript $\pi$ to denote that the Bellman equation costs used are those of that policy, i.e., $V_{t, \pi}$. Also note that the costs we consider in the integrated objective in (6.16a) are the drayage costs only, i.e., $z_{t}^{\mathrm{D}}$, such that the long-haul costs are not added twice since they are already included in $z_{t}^{\mathrm{L}}$. The function $\Gamma$ maps the probabilities of the drayage $\mathcal{P}^{\mathrm{D}}$, and the drayage decisions that consider the long-haul $\left[x_{t, \pi}^{\mathrm{D}}\right]_{\forall t \in \mathcal{T}}$ under policy $\pi$, to the probabilities of the long-haul $\mathcal{P}_{\pi}^{\mathrm{L}}$ under policy $\pi$, as shown in (6.16c). It is in these two components that the recursion lies: to compute $\mathcal{P}_{\pi}^{\mathrm{L}}$, we need $x_{t, \pi}^{\mathrm{D}}$, which depends on $V_{t, \pi}$, which in turn depends on $\mathcal{P}_{\pi}^{\mathrm{L}}$, as illustrated in Figure 6.3.

In Figure 6.3, we see that drayage decisions are made using long-haul costs and long-haul freights. These long-haul costs and long-haul freights are dependent on the MDP policy $\pi$, which is determined using freight arrival probabilities depending on the drayage decisions. When determining an MDP policy, drayage decisions that depended on long-haul costs from the MDP policy need to be included. This means that, in our integrated scheduling of drayage operations and long-haul transport, the recursion is present when determining the MDP policy (i.e., computing the value functions $V_{t, \pi}$ ). Once the value functions are computed, they do not change with daily long-haul decision-making in practice (i.e., the recursion is not present when implementing in practice). Considering this recursion, we have two approaches to solve the problem: either we consider one optimization problem with a large system of equations for the evolution of the network and constraints for drayage and long-haul scheduling, or we 


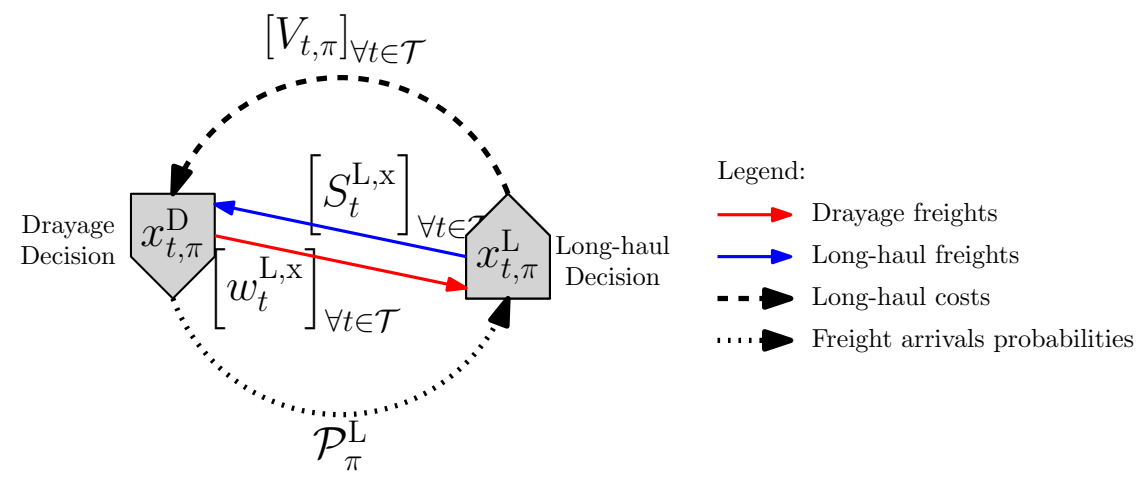

Figure 6.3: Components of the recursion between drayage and long-haul decisions

consider an iterative approach where we split the problem into several steps and iterations. Since a close-form solution for the overall optimization problem may only exist under limiting assumptions (e.g., the definition of $\Gamma$ ), we focus on an iterative approach. In the following section, we describe the iterative method for computing $\mathcal{P}_{\pi}^{\mathrm{L}}$ and solving the recursion.

\subsection{Solution Approach}

To solve the optimization problem in (6.16a), we propose a simulation-based approach connecting the MILP and MDP models for the drayage and long-haul scheduling, respectively. In this approach, we iteratively define $\mathcal{P}_{\pi}^{\mathrm{L}}$ using the costs and decisions from policy $\pi$. Our approach, which we call iterative integration, consists of four steps:

Step (A): Initialize the long-haul probabilities $\mathcal{P}_{\pi}^{\mathrm{L}}$ (in case of the first iteration), or update them using a function $\Gamma$ as in $(6.16 \mathrm{c})$.

Step (B): Solve the Bellman equations in (6.14) using the exogenous information from the probabilities $\mathcal{P}_{\pi}^{\mathrm{L}}$ resulting from Step (A). Store the expected long-haul costs $V_{t, \pi}\left(s_{t}^{\mathrm{L}}\right)$ for all states and days in the horizon.

Step (C): Simulate the drayage and long-haul scheduling policies resulting from the Bellman equations, for the entire horizon, and for a number of replications. Each replication yields an observation of drayage and long-haul costs, $\hat{z}_{\pi}^{\mathrm{D}}$ and $\hat{z}_{\pi}^{\mathrm{L}}$, respectively, as well as an observed set of drayage decisions $\left[x_{t, \pi}^{\mathrm{D}}\right]_{\forall t \in \mathcal{T}}$.

Step (D): Decide whether to continue to the next iteration and go back to Step (A) or to stop.

The four steps of the iterative integration method are shown in Figure 6.4. In addition, we show a second approach which we call the sequential integration method. This method consists of Steps (A) and (B) of the iterative integration only, or in other words, only a 
single iteration. This method is computationally simpler and can be used, for instance, when there is an analytical expression to define the drayage-dependent arrival rates for each terminal, or when these probabilities can be defined based on experience of human planners or historical data. We use this second approach for our experiments, as we explain later on in Section 6.5. For now, we focus on the challenges of the iterative integration.

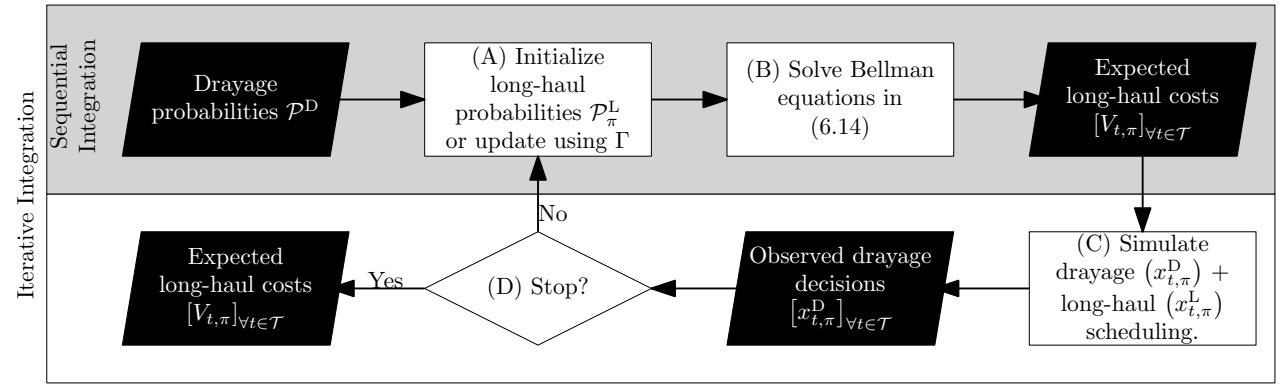

Figure 6.4: Proposed solution methods to the integrated scheduling model

Each step of the iterative integration has its own challenges. Steps (A) and (D) have challenges that are intrinsic to the simulation-based approach connecting the MILP and MDP models. First, defining long-haul probabilities $\mathcal{P}_{\pi}^{\mathrm{L}}$ involves defining the function $\Gamma$. Second, deciding whether to stop or to continue involves the assessment of whether the policy $\pi$ is good enough, or if it converged (i.e., continuing does not have any effect). Steps (B) and (C) involve the computational challenges due to increasing complexity of the MILP and MDP models with increasing problem size. Although these challenges can be tackled using heuristic approaches, these heuristics must be tuned in accordance to the new integrated problem being considered, i.e., the specifications of the models from Section 6.3.1 and 6.3.2. We elaborate on these challenges in the remainder of this section. In Section 6.4.1 we discuss the modeling challenges of Steps (A) and (D), and in Section 6.4.2 we discuss the computational challenges of Steps (B) and (C). We finalize with a heuristic design for our solution approach.

\subsubsection{Modeling Challenges}

Steps (A) and (D) are the fundamental steps where our iterative approach defines the information parameters that integrate drayage and long-haul decisions shown in Figure 6.3: the long-haul freight arrival information $\mathcal{P}_{\pi}^{\mathrm{L}}$ and the drayage terminal assignment cost $\left[V_{t, \pi}\right]_{\forall t \in \mathcal{T}}$. The general objective in these steps is to define the parameters that achieve the overall objective in (6.16).

In Step (A), a function $\Gamma$ that maps the probabilities of drayage freight arrivals $\mathcal{P}^{\mathrm{D}}$ to the probabilities of the long-haul freight arrivals $\mathcal{P}_{\pi}^{\mathrm{L}}$ must be defined. The challenge is two-fold. First, the dependence of $\mathcal{P}_{\pi}^{\mathrm{L}}$ in the drayage decisions that consider the long-haul $\left[x_{t, \pi}^{\mathrm{D}}\right]_{\forall t \in \mathcal{T}}$ is recursive, and hence the mapping complex to define. Second, the influence of $\mathcal{P}_{\pi}^{\mathrm{L}}$ in 
the overall costs/objective is indirect and difficult to measure. For the long-haul costs, the MDP model yields the expected costs of $\mathcal{P}_{\pi}^{\mathrm{L}}$, but for the drayage costs and the integrated costs the relation depends on difficult relations between the MILP and the MDP model. This difficulty is also present in Step (D). In Step (D), a decision whether to continue to the next iteration must be made. The main criteria is whether the policy $\pi$ from $\mathcal{P}_{\pi}^{\mathrm{L}}$ result in sufficiently low overall costs. However, as stated before, this is difficult to measure, and hence other mechanisms may be necessary, such as convergence of costs and decisions.

\subsubsection{Computational Challenges}

In contrast to the modeling challenges in Steps (A) and (D), Steps (B) and (C) have computational challenges with increasing problem size due to the complexity in solving the individual MILP and MDP models. Per iteration, the MDP model has to be solved once, and the MILP model $|\mathcal{T}| * N$ times, where $N$ is the number of simulation runs and $|\mathcal{T}|$ the length of the planning horizon, per iteration. To overcome these challenges, we use the MathHeuristic (MH) for the MILP model from Chapter 5 (Section 5.5) and the Approximate Dynamic Programming (ADP) algorithm for the MDP model from Chapter 4 (Section 4.5). We apply two modifications to the $\mathrm{MH}$ and one modification to the ADP algorithm, as follows.

In the ADP algorithm, we modify the Value Function Approximation (VFA) by designing a new set of characteristics $\mathcal{B}_{t}$, or basis functions $\phi_{b} \forall b \in \mathcal{B}_{t}$, that approximate the downstream rewards in the Bellman's equations. This new VFA provides a better fit with the integrated problem and works as follows. We categorize freights based on a common freight description used in transport problems: must go and may go freights. On a given day $t$, must go freights are those freights due at $t$, i.e., time-window length equal to zero, and may go freights are those freights that can be postponed, i.e., time-window length greater than zero. Based on this categorization, we include the following four basis functions per terminal $j \in \mathcal{H}$ : (i) number of must go freights, (ii) count of must go destinations, (iii) number of may go freights, and (iv) count of may go destinations, as shown in (6.17a) to (6.17d), respectively. To count the must go destinations, we use the binary variables $u_{j, d, t}$, which get a value of 1 if there is a must go freight to destination $d \in \mathcal{D}^{\mathrm{L}}$ in terminal $j \in \mathcal{H}$ and 0 otherwise. Similarly, we use the binary variable $v_{j, d, t}$ to count the may go destinations. In addition to the must go and may go freight basis functions, we include the total number of freights of a post-decision state in $(6.17 \mathrm{e})$, a constant in (6.17f), and all post-decision state variables, as shown in $(6.17 \mathrm{~g})$. In total, we now have $\left|\mathcal{B}_{t}\right|=\left|s_{t}^{\mathrm{L}}\right|+6$ characteristics (or features as we called them in previous chapters), which are indexed from $\left[1,2, \ldots,\left|\mathcal{B}_{t}\right|\right]$. Note that in $(6.17 \mathrm{~g})$, the function $b(d, l, t)$ provides an index number in the range $\left[7,8, \ldots, 7+\left|s_{t}^{\mathrm{L}}\right|\right]$.

$$
\begin{gathered}
\phi_{1, j}\left(s_{t}^{\mathrm{L}}\right)=\sum_{d \in \mathcal{D}^{\mathrm{L}}} f_{j, d, 0, t}^{\mathrm{L}} \\
\phi_{2, j}\left(s_{t}^{\mathrm{L}}\right)=\sum_{d \in \mathcal{D}^{\mathrm{L}}} u_{j, d, t} \\
\phi_{3, j}\left(s_{t}^{\mathrm{L}}\right)=\sum_{d \in \mathcal{D}^{\mathrm{L}}} \sum_{l \in \mathcal{T} \backslash 0} f_{j, d, l, t}^{\mathrm{L}}
\end{gathered}
$$




$$
\begin{gathered}
\phi_{4, j}\left(s_{t}^{\mathrm{L}}\right)=\sum_{d \in \mathcal{D}^{\mathrm{L}}} v_{j, d, t} \\
\phi_{5, j}\left(s_{t}^{\mathrm{L}}\right)=\sum_{d \in \mathcal{D}^{\mathrm{L}}} \sum_{l \in \mathcal{T}} f_{j, d, l, t}^{\mathrm{L}} \\
\phi_{6, j}\left(s_{t}^{\mathrm{L}}\right)=1 \\
\phi_{b(d, l, t), j}\left(s_{t}^{\mathrm{L}}\right)=f_{j, d, l, t}^{\mathrm{L}}, \quad \forall d \in \mathcal{D}^{\mathrm{L}}, l \in \mathcal{T}
\end{gathered}
$$

The VFA is then the product of the basis functions and weights $\theta_{b, j, t}$ as shown in (6.18). This VFA is used in the drayage costs that consider the incremental long-haul $\operatorname{costs} C_{t}^{\mathrm{L}}(\cdot)$ shown in (6.15), which depend on the state $s_{t}^{\mathrm{L}}$ and $s_{t-1}^{\mathrm{L}, \mathrm{x}}$. The increment $C_{t}^{\mathrm{L}}(\cdot)$ captures the extra costs in the long-haul when bringing drayage freight to the terminals, and in this case, these extra costs are based on the approximated downstream costs of the individual terminals (i.e., terminal dependent VFA). Thus, the weights are not used to estimate the downstream costs of having a given group of freights at a terminal, but the marginal increase in downstream costs due to this group of extra freights.

$$
C_{t}^{\mathrm{L}}\left(f_{t}^{\mathrm{L}}, x_{t}^{\mathrm{D}}\right)=V_{t}\left(s_{t}^{\mathrm{L}}\right)-V_{t}\left(s_{t-1}^{\mathrm{L}, \mathrm{x}}\right) \approx \sum_{j \in \mathcal{H} b \in \mathcal{B}} \theta_{b, j, t} \cdot \phi_{b, j}\left(s_{t}^{\mathrm{L}}\right)-\sum_{j \in \mathcal{H} b \in \mathcal{B}} \theta_{b, j, t} \cdot \phi_{b, j}\left(s_{t-1}^{\mathrm{L}, \mathrm{x}}\right)
$$

For the MH approach to solve the MILP model, we include one additional Math-Heuristic Operator (MHO). This MHO is based on the long-haul performance resulting from the VFA described before, as shown in (6.19). For pre-haulage jobs $F \in \mathcal{F}_{t}$, we fix the arc to the terminal with lowest integrated costs (i.e., drayage costs and expected increase in long-haul costs), as shown in (6.19a). If the pre-haulage job needs an empty container, then schedule an empty container from the closest terminal (i.e., lowest traveling costs), as shown (6.19b).

$$
\begin{gathered}
\sum_{k \in \mathcal{K}} x_{k, i, j^{\prime}, t}=1 \\
\text { such that } \\
j^{\prime}=\underset{j \in \delta^{+}(i)}{\operatorname{argmin}}\left(T_{i, j} \cdot C_{k, i, j}^{\mathrm{VD}}+\sum_{j \in \mathcal{H} b \in \mathcal{B}} \sum_{b, j, t} \cdot \phi_{b, j}\left(s_{t}^{\mathrm{L}}\right)-\sum_{j \in \mathcal{H}} \sum_{b \in \mathcal{B}} \theta_{b, j, t} \cdot \phi_{b, j}\left(s_{t-1}^{\mathrm{L}, \mathrm{x}}\right)\right) \\
\sum_{k \in \mathcal{K}} x_{k, j^{\prime}, i, t}=1 \mid j^{\prime}=\underset{j \in \delta^{-}(i)}{\operatorname{argmin}}\left(T_{j, i} \cdot C_{k, i, j}^{\mathrm{VD}}\right)
\end{gathered}
$$

\subsubsection{Heuristic Approach}

With the MH and ADP heuristics, our iterative integration method works as shown in Figure 6.5. First, ADP learns the VFA (i.e., weights $\theta$ of the basis functions described in the previous sections) based on the initial long-haul arrival probabilities $\mathcal{P}^{\mathrm{L}}$. Then, a simulation is carried out where freights arrive randomly for the drayage. These freights are scheduled 


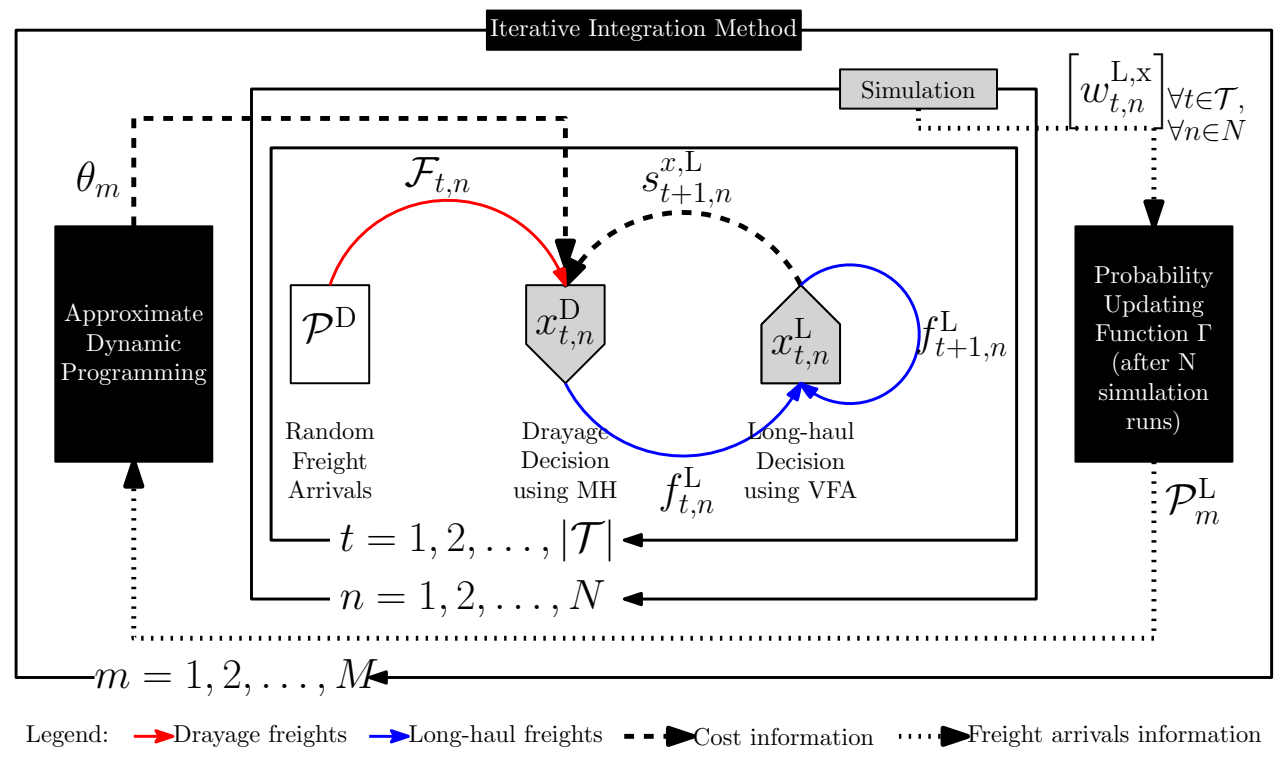

Figure 6.5: Graphical representation of the iterative integration method

using the $\mathrm{MH}$ described in the previous section, considering the learned VFA. After $N$ simulation runs, the observed long-haul arrivals $w^{\mathrm{L}, \mathrm{x}}$ for all days in the horizon and simulation runs are given to the probability updating function $\Gamma$, which yields new long-haul arrival probabilities. The process is repeated $M$ times. Note that in Figure 6.5, parameters that vary per simulation run have a subscript $n$, and those that vary per iteration of the integrated approach (but remain constant through the simulation) have a subscript $m$. Furthermore, the ADP algorithm runs for a number of internal-iterations at each iteration $m$ and the $\mathrm{MH}$ runs for a number of internal-iterations at each time $t$, of replication $n$, and of iteration $m$.

\subsection{Numerical Experiments}

In this section, we study the calibration and performance of our integrated scheduling approach through a series of numerical experiments. We study the behavior of two methods to define the long-haul probabilities and various settings for the ADP algorithm. Furthermore, we evaluate the gains of our integrated scheduling approach over independent scheduling, under various problem characteristics. Before presenting the results of our experiments, we describe our experimental setup. 


\subsubsection{Experimental Setup}

We consider a transport network with a point-to-point topology and three long-haul services, as shown in Figure 6.6. The network spans an area of $1000 \times 500 \mathrm{~km}$, to resemble the distances that make consolidation (e.g., using barges and trains) for the long-haul desirable in Europe [116]. The origin of pre-haulage freights, as well as all other drayage request locations, occurs in an area of $200 \times 500 \mathrm{~km}$ around Terminals 1,2 , and 3 . There are 25 trucks, of which 9 are located at Terminal 1, and 8 at each of the Terminals 2 and 3. There are six long-haul destinations for pre-haulage freights, which are evenly distributed between Terminals 4 , 5 , and 6 . There is the possibility to hire additional trucks for the long-haul, at a much larger expense than the trains and barges in the network. We use a similar cost structure as in Chapter 4 (Section 4.6.1), where drayage operations, on average, contribute $40 \%$ to the total transport costs. The cost details and parameters are shown in Appendix 6-B.

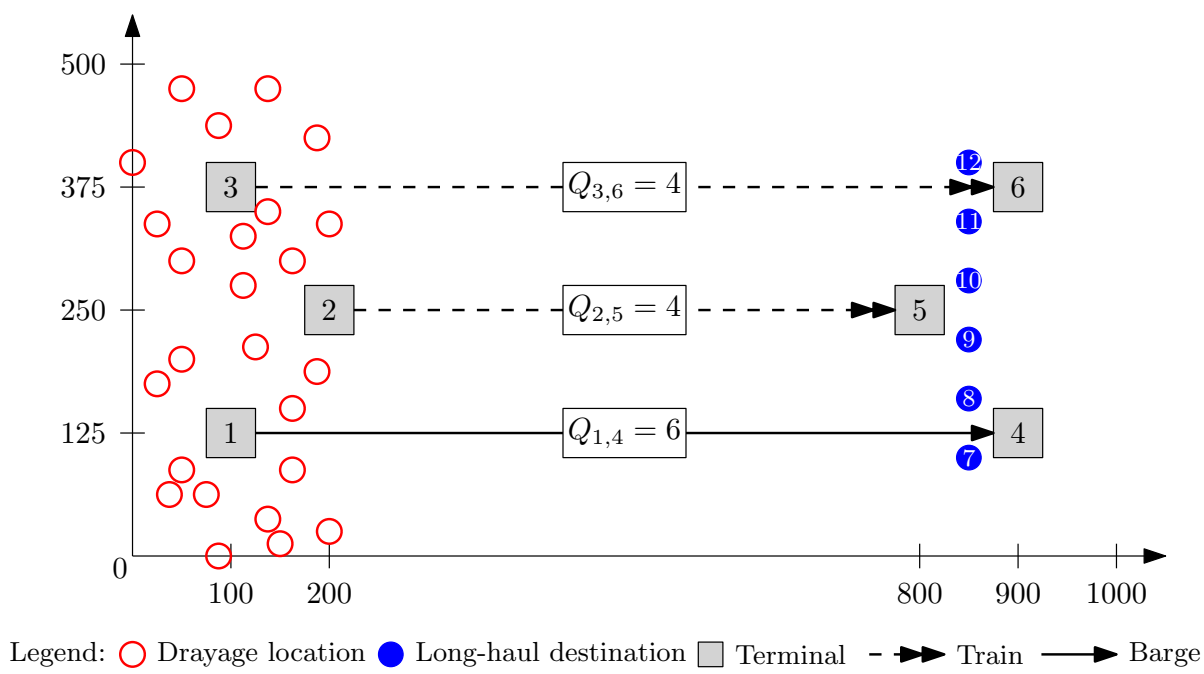

Figure 6.6: Locations and transport modes of the experimental network

We consider a planning horizon of five days, i.e., one working week. Each day, new drayage freights arrive according to a Poisson distribution with mean 20 and with a cap of 25 , where we assign the probability mass of values larger than 25 to the probability mass of the mean. Each freight that arrives has the following random characteristics:

Drayage location. The drayage location refers to the origin of pre-haulage freights and

the destination of end-haulage freights. We test two settings for the probability distribution of drayage locations. In the random setting, drayage locations are uniformly distributed in an area of $200 \times 500 \mathrm{~km}$ around Terminals 1,2 , and 3, as exemplified in Figure 6.6. In the clustered setting, drayage locations are uniformly distributed in a square of $150 \mathrm{x} 150 \mathrm{~km}$ around Terminal 3 only. 
Drayage request type. For the Drayage Request (DR) type, we consider four categories as follows. Category $D R 1$ represents an end-haulage request starting with a full container at a known terminal, transporting it to a customer (i.e., drayage location), unloading it, and then finishing with the transport of the empty container to any terminal. Category $D R 2$ represents an end-haulage request starting with a loaded container at a known terminal and finishing with its transport to the customer. Category DR3 represents a pre-haulage request starting with an empty container in any of the terminals, transporting it to a customer, loading it, and then finishing with the transport of the loaded container to any terminal. Category $D R_{4}$ represents a pre-haulage request starting with a loaded container at a customer and finishing with its transport to any terminal. We test two settings for the probability distribution of drayage request types. In the pre-haulage dominant setting, a drayage request is category DR1 with probability 0.1 , DR2 with 0.1 , DR3 with 0.4 , and DR4 with 0.4. In the end-haulage dominant setting, a drayage request is category DR1 with probability 0.4, DR2 with 0.4, DR3 with 0.1, and DR4 with 0.1.

Long-haul destination. The long-haul destination refers to where the a pre-haulage freight must be delivered after its long-haul, independent of its type (i.e., DR3 or DR4). We test two settings for the probability distribution of long-haul destinations. In the unbalanced setting, each pre-haulage freight that arrives has long-haul destination 7,8 , 9 , or 10 with probability 0.05 each, and destination 11 or 12 with probability 0.4 each. In the balanced setting, each long-haul destination has probability of $1 / 6$.

The aforementioned characteristics of demand have a direct impact on the costs of the drayage operations and the long-haul transport. For the purpose of analyzing the sensitivity of our integrated scheduling approach to these characteristics, we use two different settings for each, as shown in Table 6.1 and described before. We define an instance as a combination of the settings for each random characteristic (i.e., eight combinations or instances in total). We denote an instance using three letters from Table 6.1, e.g., R-P-U stands for an instance with random drayage locations, pre-haulage dominant drayage request types, and unbalanced long-haul destinations.

Table 6.1: Random characteristics of demand, their settings, and notation.

\begin{tabular}{ll}
\hline Characteristic & Settings (Notation) \\
\hline Drayage location & Random (R), Clustered (C) \\
Drayage request type & Pre-haulage dominant (P), End-haulage dominant (E) \\
Long-haul destination & Unbalanced (U), Balanced (B) \\
\hline
\end{tabular}

The remaining settings of our experiments are as follows. We consider that each prehaulage freight has a long-haul time-window length of 1 or 3 days with probability 0.1 each, and 2 days with probability 0.8 . The drayage time-window length, maximum driving time of drayage trucks, and terminal opening times are equal to one day. We divide the experiments into two phases. First, a calibration phase where we study the tuning of four parameters: (i) ADP initial probability distributions, (ii) ADP time horizon, (iii) ADP initial weights, and (iv) ADP update step size. These parameters have a direct influence on the expected long-haul 
costs for the planning horizon, which affect the terminal assignment decision in the drayage and consequently the freights available for consolidation in the long-haul. We describe the details of these experiments and their results in Section 6.5.2. Second, an evaluation phase where we study the costs savings of our integrated approach compared to the costs of using a non-integrated approach commonly found in practice. We describe the details of these experiments and their results in Section 6.5.3. For computational reasons, in both phases we study the sequential integration method (see Figure 6.4), which can be seen as a special case of the iterative integration method in Figure 6.5 with $M=1$. After the two phases, we discuss the limitations of our experiments and provide future research ideas to overcome them in Section 6.6.

\subsubsection{Calibration Experiments}

In the calibration experiments, we study the relation between the tunable parameters of our integrated scheduling approach and the total costs. We do not strive to find the best setting possible for each parameter, but strive to study the sensitivity of the total costs for each parameter, under different network characteristics. Before presenting the results, we describe the tunable parameter values tested.

We test a total of 72 combinations of tunable parameter settings as follows. For the initial long-haul probability distributions for each terminal, we test two possible settings: an even distribution, where the number of freights are split evenly among the terminals and all other characteristics are the same as the overall distributions, and a "cost-oriented" distribution, which differs from the even distribution only in the distribution of destinations per terminal. We describe this distribution as cost-oriented because destinations closer to the end of the long-haul service of a terminal, i.e., cheapest destination to visit from a terminal, obtain $80 \%$ of the probability mass. These distributions can be found in Appendix 6-C. For the ADP time horizon, we use two settings: equal time horizon (i.e., equal to the problem horizon of 5 days), or a larger time horizon of 7 days. For the initial weights of the VFA in ADP, we use a "slope-oriented" setting, where all weights are equal to 1, or an "intercept-oriented" setting, where all weights are equal to 0 except for the constant, which is equal to $1.2 \times 10^{4}$. For the step size $\lambda$ used by ADP in the non-stationary least square updating method, we test 9 values, equally distributed between 0.1 and 0.9 . These tunable parameters, and the notation we use to refer to them in the results, are shown in Table 6.2. The ADP algorithm runs for a total of 500 internal-iterations and the matheuristic runs for a single internal-iteration with the additional operator described in Section 6.4. Note that these iterations are from the heuristic themselves, and not with the iterations $M$ of the iterative integration method.

For each instance, we test the 72 combinations of tunable parameters using the sequential integration method. First, we run the ADP algorithm with a given set of parameters, and learn the weights of the basis functions. Second, we run a simulation of $M=100$ replications of the time horizon and include the so-called "end effects", which are costs for the long-haul freights that were postponed at each terminal at the end of the horizon, when running each replication of a given policy. Each step uses common random numbers across parameter combinations in order for performance differences to arise from the difference in tunable parameters. We measure three performance indicators: drayage costs, long-haul costs, and total costs (i.e., the sum of the two). In the following, we summarize the results of these $8 \times 72$ experiments. 
Table 6.2: Tunable parameters and their values

\begin{tabular}{ll}
\hline Tunable Parameters & Values (code) \\
\hline ADP initial probability distributions & Even, Cost-oriented \\
ADP time horizon & Equal (T5), Longer (T7) \\
ADP initial weights & Slope-oriented (W1), Intercept-oriented (W2) \\
ADP update step size & $0.1,0.2, \ldots, 0.9$ \\
\hline
\end{tabular}

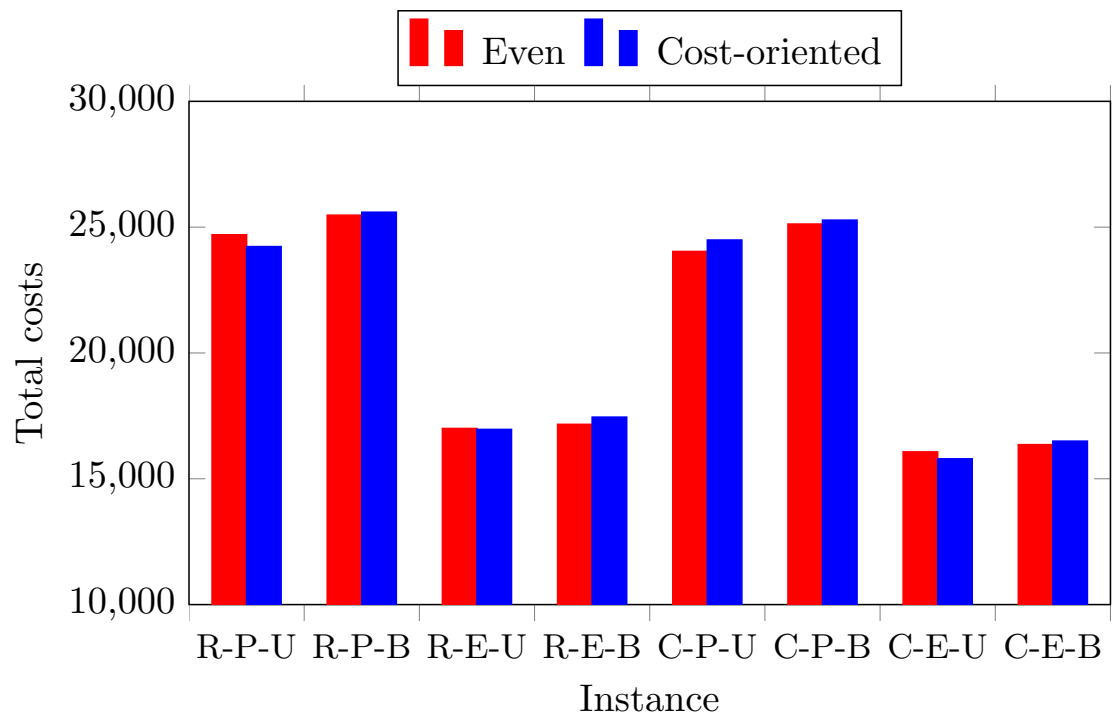

Figure 6.7: Total costs, per instance, for the best ADP settings of the even and cost-oriented probability distributions among terminals.

In Figure 6.7, we show the differences between the even and cost-oriented setting for the initial long-haul probability distributions. For each instance in this figure, we show the best settings of the remaining three tunable parameters, i.e., the combination of parameters that achieves the lowest total costs. Two important observations arise. First, the differences between the two initial probability distributions is not large. However, the even distribution is slightly better in 5 out of the 8 instances. It seems that, given that all other ADP parameters are tuned, the initial distribution does not play a significant role in achieving the lowest total costs. We come back to this issue in Section 6.6. Second, total costs are significantly larger, on average $49 \%$, in instances with pre-haulage dominant drayage types than in end-haulage drayage types. Naturally, pre-haulage dominant instances have larger long-haul costs than the end-haulage dominant instances because of the longer paths of 
freights (see Figure 6.2). To study the relation between tunable parameters of ADP for the long-haul cost and for the drayage cost, we look at three individual instances with the even initial distribution: Instance R-P-U, C-P-U, and C-E-U. We look into the relation of all other tunable parameters to the drayage, long-haul, and total costs in Figure 6.8 through 6.13.

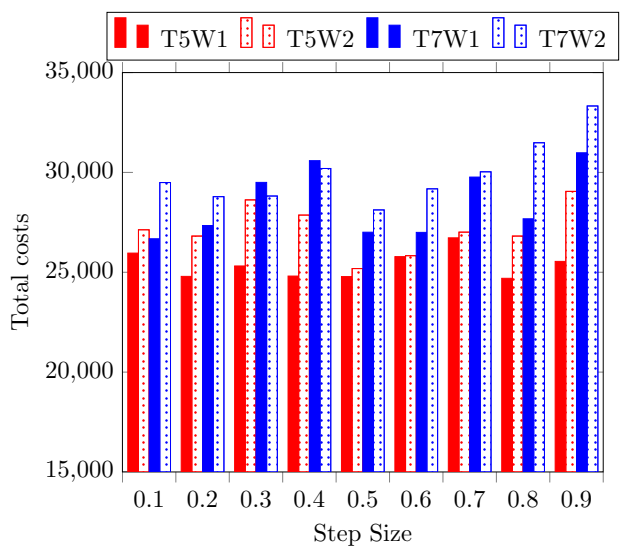

Figure 6.8: Total Costs $R-P-U$

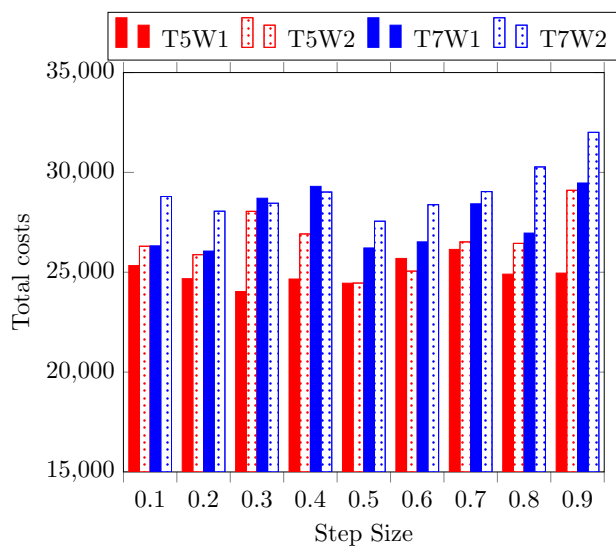

Figure 6.10: Total Costs $C-P-U$

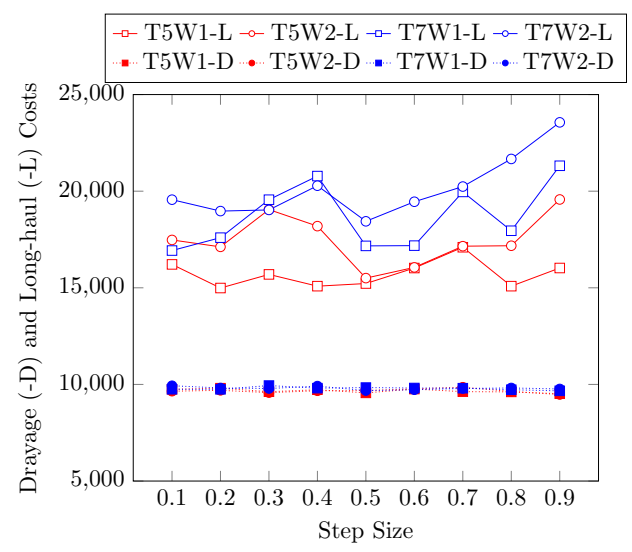

Figure 6.9: Individual Costs $R-P-U$

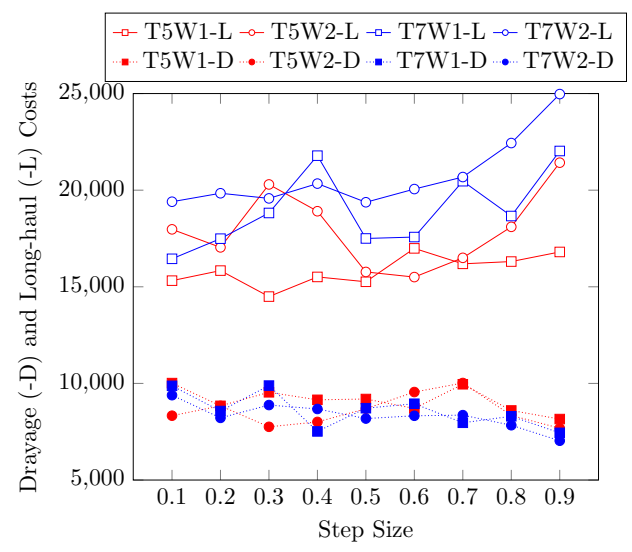

Figure 6.11: Individual Costs $C-P-U$ 


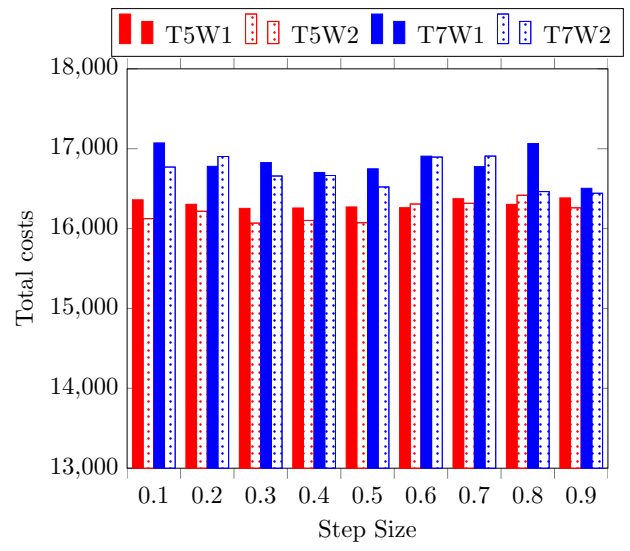

Figure 6.12: Total Costs $C-E-U$

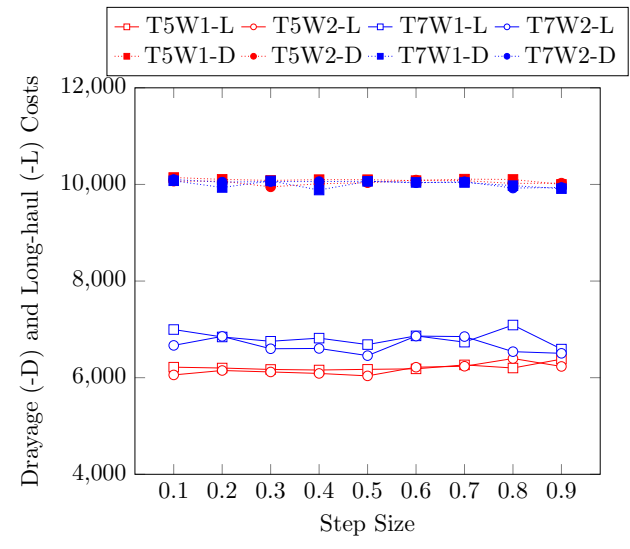

Figure 6.13: Individual Costs $C-E-U$

For Instance R-P-U, we observe in Figure 6.8 that having an equal ADP time horizon (T5) and constant initial weights (W1) are the best settings to achieve lower total costs. In Figure 6.9, we observe that T5W1 obtain the lowest total costs in R-P-U by achieving significantly lower long-haul costs than the other settings without increasing the drayage costs. In a similar way, settings T5W1 achieve the lowest total costs for Instance C-P-U, as shown in Figure 6.10. However, in contrast to R-P-U, we observe that T5W1 obtains the lowest total costs by increasing drayage costs compared to other settings. This behavior is to be expected, considering that this instance has clustered drayage locations around Terminal 3, while freights to some long-haul destinations are transported cheaper in other terminals. In other words, drayage operations have to incur additional travel costs to bring freight to other terminals in order to have lower costs for the long-haul of some destinations.

For Instance C-E-U, in Figure 6.12, we observe that differences in total cost among different tunable parameters are not as large as in the previous instances (around 1000 rather than 5000) and that the best settings are those with equal time horizon (T5) and slope-oriented initial weights (W2). In Figure 6.13, we observe that the savings in total costs come again from the long-haul costs without significantly increasing drayage costs. Furthermore, we observe that long-haul costs are lower than drayage (which is to be expected due to more end-haulage requests), explaining the smaller total costs differences across settings than in the previous instances. In the remaining instances, we observe similar behavior with respect to long-haul and drayage cost relation and best settings to achieve the lowest total costs. For all instances, having an equal ADP time horizon (T5) was the best setting. For instances with pre-haulage dominant drayage types, the constant initial weights (W1) and a step-size of 0.4 were the best settings; while for instances with end-haulage dominant types, the slope-oriented initial weights (W2) and a step-size of 0.1 were the best settings. We use these settings in the remainder of the experiments. 


\subsubsection{Evaluation Experiments}

In the evaluation experiments, we evaluate the cost savings of our integrated scheduling approach over a non-integrated scheduling approach. The non-integrated scheduling approach consists of two independent heuristics, one for the long-haul and one for the drayage. The longhaul heuristic consolidates freights that must be sent today with the lowest cost possible (i.e., combination of high-capacity modes and hired trucks), and, if there is capacity left in the highcapacity mode, it schedules freights that do not add extra costs, such as freights with the same destination. The drayage heuristic uses the logic from [12]: first, it pairs drayage requests that are close to each other (i.e., assign closest terminal), second it adds the pairs to a route using cheapest insertion, and finally schedules all remaining requests using cheapest insertion. Our integrated approach uses the best settings described in the previous section for the eight instances tested. Furthermore, for each of the eight instances we test two additional cost setups: (i) low drayage-cost, where drayage costs make up, on average, $20 \%$ of the total costs instead of the $40 \%$ in the normal setup, and (ii) high drayage-cost, where drayage costs make up, on average, $80 \%$ of total costs. The results for the normal drayage-cost setup are shown in Table 6.3 , for the low drayage-cost setup in Table 6.4, and for the high drayage-cost setup in Table 6.3.

Table 6.3: Percentage difference with the benchmark in normal drayage-cost setup

\begin{tabular}{|c|c|c|c|c|c|}
\hline \multirow{2}{*}{ Instance } & \multicolumn{3}{|c|}{ Costs } & \multirow{2}{*}{$\begin{array}{l}\text { Long-haul } \\
\text { Utilization }\end{array}$} & \multirow{2}{*}{$\begin{array}{l}\text { Pre-haulage to } \\
\text { closest terminal }\end{array}$} \\
\hline & Total & Long-haul & Drayage & & \\
\hline R-P-U & $-2 \%$ & $-10 \%$ & $17 \%$ & $4 \%$ & $-21 \%$ \\
\hline R-P-B & $-5 \%$ & $-14 \%$ & $18 \%$ & $1 \%$ & $-27 \%$ \\
\hline R-E-U & $-32 \%$ & $-63 \%$ & $33 \%$ & $-55 \%$ & $-82 \%$ \\
\hline R-E-B & $-35 \%$ & $-65 \%$ & $32 \%$ & $-55 \%$ & $-81 \%$ \\
\hline C-P-U & $-5 \%$ & $-14 \%$ & $16 \%$ & $5 \%$ & $-37 \%$ \\
\hline C-P-B & $-6 \%$ & $-13 \%$ & $12 \%$ & $0 \%$ & $-35 \%$ \\
\hline C-E-U & $-36 \%$ & $-63 \%$ & $21 \%$ & $-56 \%$ & $-81 \%$ \\
\hline C-E-B & $-38 \%$ & $-65 \%$ & $22 \%$ & $-55 \%$ & $-82 \%$ \\
\hline
\end{tabular}

In Table 6.3, we observe that our approach achieves total costs savings between $2 \%$ and $38 \%$ in all instances. Two interesting observations arise. The first observation is that the savings are larger in instances with end-haulage dominant drayage types. These savings are the result of increased drayage costs due to pre-haulage freights not being brought to their closest terminal. Furthermore, they come at the price of lower long-haul utilization (e.g., of barges and trains). Although this might seem counter-intuitive at first, it means that our approach postpones the long-haul transport of freight (sometimes not sending any freight at all in the barge and trains) until it can consolidate the "right" freights, i.e., those that are beneficial for the long-haul costs and total costs. Considering that in instances with end-haulage dominant drayage types, only a few freights go for the long-haul, it seems reasonable to postpone the long-haul transport of freights today expecting that tomorrow more freights will arrive for consolidation. The second observation is that the savings in 
Table 6.4: Percentage difference with the benchmark in low drayage-cost setup

\begin{tabular}{|c|c|c|c|c|c|}
\hline \multirow{2}{*}{ Instance } & \multicolumn{3}{|c|}{ Costs } & \multirow{2}{*}{$\begin{array}{l}\text { Long-haul } \\
\text { Utilization }\end{array}$} & \multirow{2}{*}{$\begin{array}{l}\text { Pre-haulage to } \\
\text { closest terminal }\end{array}$} \\
\hline & Total & Long-haul & Drayage & & \\
\hline R-P-U & $-6 \%$ & $-11 \%$ & $20 \%$ & $3 \%$ & $-23 \%$ \\
\hline R-P-B & $-10 \%$ & $-16 \%$ & $19 \%$ & $2 \%$ & $-28 \%$ \\
\hline R-E-U & $-44 \%$ & $-63 \%$ & $33 \%$ & $-55 \%$ & $-81 \%$ \\
\hline R-E-B & $-47 \%$ & $-65 \%$ & $33 \%$ & $-55 \%$ & $-82 \%$ \\
\hline C-P-U & $-8 \%$ & $-14 \%$ & $19 \%$ & $4 \%$ & $-40 \%$ \\
\hline C-P-B & $-11 \%$ & $-16 \%$ & $13 \%$ & $2 \%$ & $-35 \%$ \\
\hline C-E-U & $-47 \%$ & $-63 \%$ & $22 \%$ & $-55 \%$ & $-84 \%$ \\
\hline C-E-B & $-49 \%$ & $-65 \%$ & $23 \%$ & $-55 \%$ & $-86 \%$ \\
\hline
\end{tabular}

Table 6.5: Percentage difference with the benchmark in high drayage-cost setup

\begin{tabular}{|c|c|c|c|c|c|}
\hline \multirow{2}{*}{ Instance } & \multicolumn{3}{|c|}{ Costs } & \multirow{2}{*}{$\begin{array}{l}\text { Long-haul } \\
\text { Utilization }\end{array}$} & \multirow{2}{*}{$\begin{array}{l}\text { Pre-haulage to } \\
\text { closest terminal }\end{array}$} \\
\hline & Total & Long-haul & Drayage & & \\
\hline R-P-U & $3 \%$ & $-12 \%$ & $6 \%$ & $4 \%$ & $5 \%$ \\
\hline R-P-B & $5 \%$ & $-5 \%$ & $7 \%$ & $0 \%$ & $4 \%$ \\
\hline R-E-U & $13 \%$ & $-62 \%$ & $29 \%$ & $-55 \%$ & $-72 \%$ \\
\hline R-E-B & $12 \%$ & $-63 \%$ & $30 \%$ & $-55 \%$ & $-74 \%$ \\
\hline C-P-U & $-9 \%$ & $50 \%$ & $-20 \%$ & $-30 \%$ & $18 \%$ \\
\hline C-P-B & $-12 \%$ & $38 \%$ & $-23 \%$ & $-27 \%$ & $21 \%$ \\
\hline C-E-U & $4 \%$ & $-64 \%$ & $19 \%$ & $-55 \%$ & $-71 \%$ \\
\hline C-E-B & $3 \%$ & $-64 \%$ & $18 \%$ & $-55 \%$ & $-73 \%$ \\
\hline
\end{tabular}

the pre-haulage dominant instances are smaller than in the end-haulage dominant instances but with a better long-haul utilization. Again, the savings in total costs come from the long-haul costs and at the price of increased drayage costs.

In Table 6.4 with the low drayage-cost setup, similar observations to those from Table 6.3 arise. The only difference is in the magnitude, with larger savings being an intuitive result of drayage operations not contributing significantly to the total costs and hence the choice of transporting pre-haulage freights to the best initial long-haul terminal, rather than the closest terminal, becomes more important. However, in Table 6.5 with high drayage-cost setup, we observe two important differences. First, our approach achieves total cost savings in only 2 out of the 8 instances tested and in all others perform worse than the benchmark. These two instances, in contrast to the remaining six, have lower drayage costs but higher long-haul costs. Second, we observe that the drayage cost savings arise due to the use of more closer terminals to freight than the benchmark at the expense of the long-haul (opposite to the remaining instances). Although these observations may be due to an 
extreme situation of drayage costs being much higher than the long-haul costs, one can argue that if our approach would properly learn the VFA, and the drayage scheduling policy using the VFA is designed properly, then it would not perform worse than the non-integrated benchmark. In the following section, we discuss these observations and their implications.

\subsection{Discussion}

The results of our numerical experiments raised a series of discussion points about the integrated scheduling of drayage operations and long-haul transport. In this section, we elaborate on these points, distinguishing between experiment limitations, modeling limitations, and guidelines for further research.

Experiment limitations. For computational and compactness reasons, we did not test the iterative integration method with more than one iteration. Nevertheless, we observed that our integrated approach is capable of achieving significant savings in instances where long-haul costs are larger than drayage-costs. In instances with high drayage-costs, where we achieved savings in only 2 out of the 8 instances tested, a possible reason may be that better calibration is required for such setting (i.e., we used the ADP settings calibrated for the normal drayage-cost setup). However, for the high drayage-cost instances, one can argue that for any ADP setting, the VFA learned should have a limited impact in the drayage decisions since the largest cost component guiding these decisions is the one corresponding to the drayage operations. Yet, our experiments seem to show that the VFA plays to large of a role. We come back to this issue in the modeling limitations. Another observation with further implications was the larger percentage savings in the total costs of instances with end-haulage dominance. In these instances, our approach significantly reduced the long-haul costs, percentage wise and compared to the non-integrated benchmark, but significantly increased the drayage costs. One might argue that the scale, or weight, of the two different costs may influence the conclusion, and hence for the possible implementation in practice by an LSP, additional performance measurements than percentages are needed.

Modeling limitations. In our experiments, we observed that our model and solution approach performed well when the long-haul costs dominate, but not so well otherwise. This may be a result of our method of integration, where the drayage scheduling decisions have an explicit (i.e., direct) link to the long-haul performance but long-haul decisions only have an implicit link to the drayage. Although we did not calibrate the implicit link (i.e., probability distributions for the long-haul through the function $\Gamma$ ), and this might be one of the reasons of the under-performance, it is possible that our approach requires an explicit link for instances where the drayage costs dominate. Furthermore, other mechanisms within the ADP algorithm used for Step (B) in our approach may require further designs when used in an integrated approach. For example, it could be that the VFA learned is not able to give a good estimate of having some freight at some terminal in the simulation phase of our integrated approach due to the initial state and probability distribution used for learning. Consider, for example, a VFA that was learned under an initial state having 3 freights and under a probability distribution saying that at most 7 freights arrive to a given terminal per day. During the learning phase, the day after the initial day will never have more than 10 freights 
(and similarly with subsequent days). During the implementation phase (simulation in our case), however, it can happen that this VFA is used to estimate the costs of bringing more than 10 freights. Although the basis functions in our VFA can give an estimate due to their parameterized nature, this estimate is not reliable since it was never observed/used during the learning phase. Adding this unreliability to the fact that there are high drayage-costs, the comparison of bringing more than 10 freights to this terminal might not at all be reliable.

Further research. The first guideline for further research is to test the iterative integration approach. This involves four challenges: (i) how to make the computational burden smaller, (ii) how to define a function $\Gamma$ in Step (A) that captures drayage performance the best, especially when the cost emphasis is on the drayage operations, (iii) how to further design VFA learning mechanisms (e.g., randomized initial states) for ADP in Step (B) that are able to cope with situations that will be faced upon implementation, and (iv) how to decide whether to continue to the next iteration in Step (D) analyzing the convergence of probabilities, costs, and the scheduling policy resulting from the VFA. The second guideline is to explore other characteristics that may have an influence on the cost saving opportunities. In our experiments, the type of drayage jobs (i.e., pre-haulage or end-haulage dominant) had a larger influence than the location of the requests or the destination of the long-haul. Perhaps other time-related characteristics, which we assumed fixed in our study, have an important influence on the savings that our integrated scheduling approach can achieve.

\subsection{Conclusions}

In this chapter, we designed a simulation-based approach to integrate a MILP model for scheduling drayage operations and an MDP model for scheduling long-haul transport. With our approach, we studied the relation between scheduling decisions, timing, and overall performance of drayage and long-haul operations in synchromodal transport considering stochastic demand and multiple freight characteristics. In addition, we tested how our integrated scheduling approach could be calibrated and how it performed compared to a benchmark heuristic over various network configurations.

We showed that our integration approach results in significant savings compared to a nonintegrated approach: between $2 \%$ to $38 \%$ under normal cost settings over all instances, and between $6 \%$ and $49 \%$ in case of low drayage costs. In high drayage cost settings, however, we only achieved savings in 2 out of the 8 instances tested. These savings, as well as the instances were our approach was more expensive, helped us identify two areas for further research. First, an iterative definition of the long-haul probabilities for the ADP algorithm that allows the right emphasis on the drayage operations, as well as a learning mechanisms that cope with situations faced upon implementation, must be designed. Second, time and cost characteristics of the freight demand must be studied in more detail to provide insight into the applicability of our method in practice. We observed that the savings of our approach had significant implications to either the drayage operations or the long-haul transport. Studying these implications further will achieve improvements for the practice of synchromodal transport through the integrated scheduling of drayage operations and long-haul transport. 


\section{Appendices}

\section{6-A Notation}

We divide the notation into four categories: (i) sets, (ii) probabilities, (iii) parameters, and (iv) variables. For clarity purposes, some of the notation is repeated in more than one category. In all of the notation, a superscript ' $\mathrm{D}$ ' indicates that the parameter or variable is related to drayage scheduling, while the superscript ' $\mathrm{L}$ ' indicates a relation to long-haul scheduling.

Table 6.6: Sets and their elements/indices ( ${ }^{*}$ indicates miscellaneous elements).

\begin{tabular}{|c|c|c|}
\hline Set & Indices & Description \\
\hline $\mathcal{T}$ & $t, t^{\prime}$ & Long-haul scheduling periods (i.e., planning horizon). \\
\hline $\mathcal{F}_{t}$ & $f^{\mathrm{N}}$ & $\begin{array}{l}\text { Freights that arrived between the drayage scheduling of } t-1 \\
\text { and } t \text {. }\end{array}$ \\
\hline $\mathcal{R}$ & $r, r^{\mathrm{D}}$ & Drayage request types of freights. \\
\hline $\mathcal{O}$ & $o, o^{\mathrm{D}}$ & Drayage origins of freights. \\
\hline $\mathcal{D}^{\mathrm{D}}$ & $d^{\mathrm{D}}$ & Drayage destinations of freights. \\
\hline $\mathcal{D}^{\mathrm{L}}$ & $d^{\mathrm{L}}$ & Long-haul destinations of freights. \\
\hline $\mathcal{T}^{\mathrm{D}}$ & $e^{\mathrm{D}}, l^{\mathrm{D}}$ & $\begin{array}{l}\text { Drayage time-windows, where } e^{\mathrm{D}} \text { is earliest arrival and } l^{\mathrm{D}} \\
\text { is latest arrival. }\end{array}$ \\
\hline $\mathcal{T}^{\mathrm{L}}$ & $l^{\mathrm{L}}$ & Long-haul time-window lengths of freights. \\
\hline $\mathcal{P}^{\mathrm{D}}$ & Misc.* & $\begin{array}{l}\text { Set of all probabilities describing the arrival of freight for } \\
\text { drayage. }\end{array}$ \\
\hline $\mathcal{P}^{\mathrm{L}}$ & Misc.* $^{*}$ & $\begin{array}{l}\text { Set of all probabilities describing the arrival of freight to } \\
\text { the long-haul terminals. }\end{array}$ \\
\hline $\mathcal{K}$ & $k$ & Set of drayage trucks. \\
\hline $\mathcal{K}_{r}$ & $k$ & $\begin{array}{l}\text { Set of drayage trucks that can carry our drayage request } \\
\text { type } r \in \mathcal{R} \text {. }\end{array}$ \\
\hline $\mathcal{H}$ & $h$ & Set of all terminals available in the network. \\
\hline $\mathcal{N}^{\mathrm{D}}$ & $i, j$ & Set of all locations in the drayage. \\
\hline $\mathcal{N}^{\mathrm{L}}$ & $i, j$ & Set of all locations in the long-haul. \\
\hline $\mathcal{G}$ & $d$ & Subset of long-haul destinations. \\
\hline $\mathcal{X}_{t}^{\mathrm{L}}$ & $x_{t}^{\mathrm{L}}$ & Decision space of the MDP. \\
\hline $\mathcal{S}$ & $s_{t}^{\mathrm{L}}$ & State space of the MDP. \\
\hline$\Pi$ & $\pi$ & Policy space of the MDP. \\
\hline$\Omega$ & $s_{t}^{\mathrm{L}}, \omega$ & Exogenous information space of the MDP. \\
\hline $\mathcal{B}$ & $b$ & Set of features for the VFA of the ADP algorithm. \\
\hline$N$ & $n$ & $\begin{array}{l}\text { Replications of the simulation in the iterative integration } \\
\text { method. }\end{array}$ \\
\hline$M$ & $m$ & Iterations in the iterative integration method. \\
\hline
\end{tabular}


Table 6.7: Probabilities

\begin{tabular}{|c|c|}
\hline Probability & Description \\
\hline$p_{f}^{\mathrm{F}}$ & $\begin{array}{l}f \in \mathbb{N} \text { freights arriving to the drayage between two consecutive } \\
\text { periods. }\end{array}$ \\
\hline$p_{r}^{\mathrm{R}}$ & Freight having drayage request type $r^{\mathrm{D}} \in \mathcal{R}$ \\
\hline$p_{o}^{\mathrm{O}}$ & Freight having drayage origin $o^{\mathrm{D}} \in \mathcal{O}$. \\
\hline$p_{r, d^{\mathrm{D}}}^{\mathrm{DD}}$ & $\begin{array}{l}\text { Freight having drayage destination } d^{\mathrm{D}} \in \mathcal{D}^{\mathrm{D}} \text {, given request } \\
\text { type } r^{\mathrm{D}} \in \mathcal{R} \text {. }\end{array}$ \\
\hline$p_{r, d^{\mathrm{L}}}^{\mathrm{DL}}$ & $\begin{array}{l}\text { Freight having long-haul destination } d^{\mathrm{L}} \in \mathcal{D}^{\mathrm{L}} \text {, given request } \\
\text { type } r^{\mathrm{D}} \in \mathcal{R} \text {. }\end{array}$ \\
\hline$p_{r, e^{\mathrm{D}}}^{\mathrm{ETD}}$ & $\begin{array}{l}\text { Freight having earliest drayage truck arrival } e^{\mathrm{D}} \in \mathcal{T}^{\mathrm{D}} \text {, given } \\
\text { request type } r^{\mathrm{D}} \in \mathcal{R} \text {. }\end{array}$ \\
\hline$p_{r, l^{\mathrm{D}}}^{\mathrm{LTD}}$ & $\begin{array}{l}\text { Freight having latest drayage truck arrival } l^{\mathrm{D}} \in \mathcal{T}^{\mathrm{D}} \text {, given } \\
\text { request type } r^{\mathrm{D}} \in \mathcal{R} \text {. }\end{array}$ \\
\hline$p_{r, l^{\mathrm{L}}}^{\mathrm{LTL}}$ & $\begin{array}{l}\text { Freight having long-haul time-window length } l^{\mathrm{L}} \in \mathcal{T} \text {, given } \\
\text { request type } r^{\mathrm{D}} \in \mathcal{R} \text {. }\end{array}$ \\
\hline $\mathcal{P}^{\mathrm{D}}$ & Set of all probabilities affecting the drayage arrivals. \\
\hline$p_{f, t}^{\mathrm{F}^{\prime}}$ & $f \in \mathbb{N}$ freights arriving to terminal $j \in \mathcal{N}^{\mathrm{L}}$. \\
\hline$p_{d, j, t}^{\mathrm{DL}}$ & $\begin{array}{l}\text { Freight arriving in terminal } j \in \mathcal{N}^{\mathrm{L}} \text { at time } t \in \mathcal{T} \text { having } \\
\text { long-haul destination } d \in \mathcal{D}^{\mathrm{L}}\end{array}$ \\
\hline$p_{l, j, t}^{\mathrm{LTL}}$ & $\begin{array}{l}\text { Freight arriving in terminal } j \in \mathcal{N}^{\mathrm{L}} \text { at time } t \in \mathcal{T} \text { having } \\
\text { time-window length } l \in \mathcal{T}^{\mathrm{L}}\end{array}$ \\
\hline $\mathcal{P}^{\mathrm{L}}$ & Set of all probabilities affecting the long-haul arrivals. \\
\hline
\end{tabular}


Table 6.8: Parameters and variables of the MILP model for the drayage operations

\begin{tabular}{cl}
\hline Parameter/Variable & Description \\
\hline$T_{r, k}^{\mathrm{SD}}$ & Time to execute a drayage request type $r^{\mathrm{D}} \in \mathcal{R}$. \\
$B_{k} \in \mathcal{H}^{\mathrm{D}}$ & Starting location of truck $k \in \mathcal{K}$. \\
$F_{k} \in \mathcal{H}^{\mathrm{D}}$ & Starting location of truck $k \in \mathcal{K}$. \\
$T_{h}^{\mathrm{SH}}$ & Service time at terminal $h \in \mathcal{H}$. \\
$T_{i, j}^{\mathrm{TD}}$ & Traveling time between locations $i$ and $j$ in $\mathcal{N}^{\mathrm{D}}$. \\
$C_{k}^{\mathrm{FD}}$ & Fixed cost for using truck $k \in \mathcal{K}$. \\
$C_{k, i, j}^{\mathrm{VD}}$ & Variable cost of truck $k \in \mathcal{K}$ for traveling between locations \\
& $i$ and $j$ in $\mathcal{N}^{\mathrm{D}}$. \\
$x_{k, i, j, t}^{\mathrm{D}}$ & Binary variable which gets a value of 1 if truck $k \in \mathcal{K}$ moves \\
$z_{t}^{\mathrm{D}}$ & from location $i$ to location $j$ in $\mathcal{N}^{\mathrm{D}}$ at time $t \in \mathcal{T}$. \\
& Drayage traveling costs at time $t \in \mathcal{T}$ (i.e., fixed plus variable \\
$C_{i, j, t}^{\mathrm{AD}}$ & traveling costs) of a drayage schedule. \\
& Cost of assigning a freight with origin $i \in \mathcal{O}$ to terminal \\
$\tilde{z}_{t}^{\mathrm{D}}$ & $j \in \mathcal{H}$ at time $t \in \mathcal{T}$. \\
& Drayage traveling and terminal assignment cost of a drayage \\
$f_{j, d, l, t}^{\mathrm{L}}$ & schedule. \\
& Number of freights at terminal $j \in \mathcal{H}$ at time $t$ that have \\
& long-haul destination $d \in \mathcal{D}^{\mathrm{L}}$ and long-haul time-window \\
$f_{t}^{\mathrm{L}}$ & length $l \in \mathcal{T}$. \\
$x_{k, i, j, t}^{\mathrm{D}}$ & Vector of variables $f_{j, d, l, t}^{\mathrm{L}}$ for all $j \in \mathcal{H}, d \in \mathcal{D}^{\mathrm{L}}, l \in \mathcal{T}$. \\
$C_{t}^{\mathrm{L}}\left(f_{t}^{\mathrm{L}}, x_{t}^{\mathrm{D}}\right)$ & Vector of variables $x_{k, i, j, t}^{\mathrm{D}}$ for all $k \in \mathcal{K}, i \in \mathcal{N}^{\mathrm{D}}, j \in \mathcal{N}$ D. \\
& Terminal assignment cost function. \\
\hline
\end{tabular}


Table 6.9: Parameters and variables of the MDP model for the long-haul transport

\begin{tabular}{|c|c|}
\hline Parameter/Variable & Description \\
\hline$Q_{i, j}$ & $\begin{array}{l}\text { Capacity, in freights, of the long-haul service going from } \\
\text { location } i \text { to } j \text {. }\end{array}$ \\
\hline$T_{i, j}^{\mathrm{TL}}$ & Duration of the long-haul service going from location $i$ to $j$. \\
\hline$C_{i, j}^{\mathrm{FL}}$ & $\begin{array}{l}\text { Fixed cost of using the long-haul service going from location } \\
i \text { to } j \text {. }\end{array}$ \\
\hline$C_{i, j, d}^{\mathrm{VL}}$ & $\begin{array}{l}\text { Variable cost of using the long-haul service going from } \\
\text { location } i \text { to } j \text { for a freight with destination } d \in \mathcal{D}^{\mathrm{L}} \text {. }\end{array}$ \\
\hline$C_{i, j, \mathcal{G}}^{\mathrm{GL}}$ & $\begin{array}{l}\text { Setup cost of using the long-haul service going from location } \\
i \text { to } j \text { for a freight whose destination } d \text { is in group } \mathcal{G} \subseteq \mathcal{D}^{L} \text {. }\end{array}$ \\
\hline$x_{i, j, d, l, t}^{\mathrm{L}}$ & $\begin{array}{l}\text { Number of freights consolidated at time } t \text { in the service } \\
\text { from } i \text { to } j \text { (both in } \mathcal{N}^{\mathrm{L}} \text { ), which have long-haul destination } \\
d \in \mathcal{D}^{\mathrm{L}} \text { and long-haul time-window length } l \in \mathcal{T} \text {. }\end{array}$ \\
\hline$y_{i, j, d, t}^{\mathrm{L}}$ & $\begin{array}{l}\text { Binary variable which gets a value of } 1 \text { if there is a freight } \\
\text { with destination } d \in \mathcal{D}^{\mathrm{L}} \text { consolidated at time } t \text { in the service } \\
\text { from } i \text { to } j \text {, both in } \mathcal{N}^{\mathrm{L}} \text {, and } 0 \text { otherwise. }\end{array}$ \\
\hline$z_{t}^{\mathrm{L}}$ & Long-haul costs of a long-haul schedule. \\
\hline$f_{j, d, l, t}^{\mathrm{L}}$ & $\begin{array}{l}\text { Number of freights at terminal } j \in \mathcal{H} \text { at time } t \text { that have } \\
\text { long-haul destination } d \in \mathcal{D}^{\mathrm{L}} \text { and long-haul time-window } \\
\text { length } l \in \mathcal{T} \text {. }\end{array}$ \\
\hline$s_{t}^{\mathrm{L}}$ & $\begin{array}{l}\text { State of the MDP: vector of variables } f_{j, d, l, t}^{\mathrm{L}} \text { for all } \\
j \in \mathcal{H}, d \in \mathcal{D}^{\mathrm{L}}, l \in \mathcal{T} \text {. }\end{array}$ \\
\hline$\hat{f}_{j, d, l, t}^{\mathrm{L}}$ & $\begin{array}{l}\text { Number of freights that arrived at terminal } j \in \mathcal{H} \text {, between } \\
\text { time } t-1 \text { and time } t \text {, that have long-haul destination } d \in \mathcal{D}^{\mathrm{L}} \\
\text { and long-haul time-window length } l \in \mathcal{T} \text {. }\end{array}$ \\
\hline$w_{t}^{\mathrm{L}}$ & $\begin{array}{l}\text { Exogenous information of the MDP: vector of variables } \\
\hat{f}_{j, d, l, t}^{\mathrm{L}} \text { for all } j \in \mathcal{H}, d \in \mathcal{D}^{\mathrm{L}}, l \in \mathcal{T} \text {. }\end{array}$ \\
\hline$S^{\mathrm{M}}$ & Transition function to capture the long-haul evolution. \\
\hline$V_{t}$ & Optimal expected costs function of the Bellman's equations. \\
\hline$s_{t}^{\mathrm{L}, \mathrm{x}}$ & Post-decision state of the MDP. \\
\hline$w_{t}^{\mathrm{L}, \mathrm{x}}$ & $\begin{array}{l}\text { New freights that arrive to the MDP based on the MILP } \\
\text { solution in the iterative integration method. }\end{array}$ \\
\hline$\Gamma$ & $\begin{array}{l}\text { Function to define the long-haul probabilities from the } \\
\text { drayage decisions in the iterative integration method. }\end{array}$ \\
\hline$\phi_{b}$ & $\begin{array}{l}\text { Basis function of feature } b \in \mathcal{B} \text { in the VFA of the ADP } \\
\text { algorithm. }\end{array}$ \\
\hline$\theta_{b}$ & Weight of the feature $b \in \mathcal{B}$ in the VFA of the ADP algorithm. \\
\hline
\end{tabular}




\section{6-B Cost Setup}

The drayage and long-haul cost setups are shown in Tables 6.10 and 6.11, respectively. The drayage fixed cost $C_{k}^{\mathrm{FD}}$ and variable cost $C_{k, i, j}^{\mathrm{VD}}$ are the same for all vehicles $k \in \mathcal{K}$. The drayage variable costs $C_{k, i, j}^{\mathrm{VD}}$ are multiplied by the traveling time $T_{i, j}^{\mathrm{TD}}$. There are two long-haul variable costs $C_{i, j, d}^{\mathrm{VL}}$ per terminal $i \in \mathcal{H}$ : the cost for using the high-capacity service (i.e., barge or train) to terminal $j$ and the cost for hiring an additional truck directly to destination $d$. Furthermore, there are three groups $\mathcal{G}$, with a group setup cost of $C_{i, j, \mathcal{G}}^{\mathrm{GL}}$ only for the high-capacity services $(i, j)$. This group setup costs captures the fixed cost component, hence we use $C_{i, j}^{\mathrm{FL}}=0$.

Table 6.10: Drayage cost setups

\begin{tabular}{cccc}
\hline Drayage Cost & Normal & Low & High \\
\hline Fixed $C_{k}^{\mathrm{FD}}$ & 200 & 100 & 2000 \\
Variable $C_{k, i, j}^{\mathrm{VD}}$ & 0.2 & 0.1 & 2 \\
\hline
\end{tabular}

Table 6.11: Long-haul costs per terminal, for all instances.

\begin{tabular}{|c|c|c|c|c|c|c|c|c|c|c|}
\hline \multirow{2}{*}{$d \in \mathcal{D}^{\mathrm{L}}$} & \multirow{2}{*}{$\mathcal{G}$} & \multicolumn{3}{|c|}{ Terminal 1} & \multicolumn{3}{|c|}{ Terminal 2} & \multicolumn{3}{|c|}{ Terminal 3} \\
\hline & & $\overline{C_{1,4, d}^{\mathrm{VL}}}$ & $C_{1,4, \mathcal{G}}^{\mathrm{GL}}$ & $C_{1, d, d}^{\mathrm{VL}}$ & $C_{2,5, d}^{\mathrm{VL}}$ & $C_{2,5, \mathcal{G}}^{\mathrm{GL}}$ & $C_{2, d, d}^{\mathrm{VL}}$ & $C_{3,6, d}^{\mathrm{VL}}$ & $C_{3,6, \mathcal{G}}^{\mathrm{GL}}$ & $C_{3, d, d}^{\mathrm{VL}}$ \\
\hline 7 & 1 & 37 & 322 & 750 & 53 & 460 & 667 & 53 & 460 & 799 \\
\hline 8 & 1 & 37 & 322 & 751 & 53 & 460 & 656 & 53 & 460 & 780 \\
\hline 9 & 2 & 66 & 425 & 756 & 46 & 298 & 651 & 66 & 425 & 766 \\
\hline 10 & 2 & 66 & 425 & 766 & 46 & 298 & 651 & 66 & 425 & 756 \\
\hline 11 & 3 & 66 & 391 & 780 & 66 & 391 & 656 & 46 & 274 & 751 \\
\hline 12 & 3 & 66 & 391 & 799 & 66 & 391 & 667 & 46 & 274 & 750 \\
\hline
\end{tabular}

\section{6-C Initial Long-haul Probability Distributions}

In this appendix, we show the "even" and "cost-oriented" initial probability distributions for the long-haul terminals. Overall, new drayage freights arrive to the system according to a Poisson distribution with mean 20 and with a cap of 25, where we assign the probability mass of values larger than 25 to the probability mass of the mean. In both, the even and cost-oriented initial distributions, we use as initial arrival distribution for terminals a Poisson distribution with mean $\frac{20}{3}$ and with a cap of 25 , where we assign the probability mass of values larger than 25 to the probability mass of the mean. The overall destination distribution for pre-haulage freights, for the unbalanced and balanced instances, is shown in Table 6.12. For the even initial probability distribution $p_{d, j, t}^{\mathrm{DL}}$ per terminal $j$ per time $t$, we use the same values of the unbalanced and balanced overall distributions, depending 
on the instance naturally. For the cost-oriented initial probability distribution, in contrast to the even, we use the distribution shown in Table 6.12, for all instances.

Table 6.12: Long-haul destination probabilities

\begin{tabular}{cccccc}
\hline & Unbalanced & Balanced & \multicolumn{3}{c}{ Cost-oriented } \\
\cline { 4 - 6 } Destination & Overall & Overall & Terminal 1 & Terminal 2 & Terminal 3 \\
$d \in \mathcal{D}^{\mathrm{L}}$ & $p_{r, d}^{\mathrm{DL}}$ & $p_{r, d}^{\mathrm{DL}}$ & $p_{d, 1, t}^{\mathrm{DL}}$ & $p_{d, 2, t}^{\mathrm{DL}}$ & $p_{d, 3, t}$ \\
\hline 7 & 0.05 & 0.166 & 0.4 & 0.025 & 0.025 \\
8 & 0.05 & 0.167 & 0.4 & 0.025 & 0.025 \\
9 & 0.05 & 0.167 & 0.025 & 0.4 & 0.075 \\
10 & 0.05 & 0.167 & 0.025 & 0.4 & 0.075 \\
11 & 0.4 & 0.167 & 0.075 & 0.075 & 0.4 \\
12 & 0.4 & 0.166 & 0.075 & 0.075 & 0.4 \\
\hline
\end{tabular}




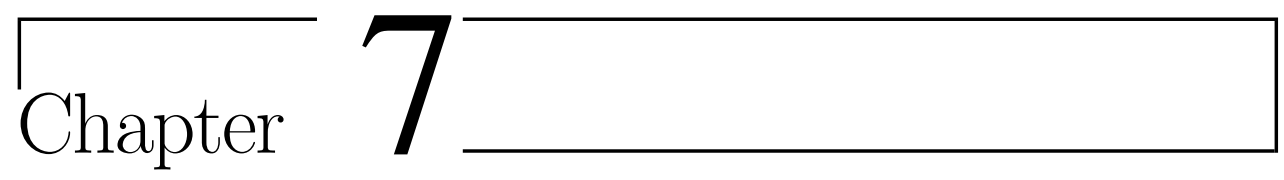

\section{Raising Awareness About Anticipatory Scheduling}

In this chapter, we explore the use of serious gaming to raise awareness about some of the trade-offs in anticipatory scheduling of synchromodal transport and to educate on how to optimize these trade-offs. We design a web-based game that simulates an LSP facing the problem from Chapter 2 and incorporates the solution algorithms developed therein. We analyze how different game scenarios and game interventions may influence the performance of players and their awareness about the trade-offs in anticipatory scheduling. The game includes dynamic leader-board that updates players about the performance of other players within the same session. We discuss how the use of our game can facilitate the adoption of an anticipatory perspective when scheduling synchromodal transport operations.

\subsection{Introduction}

Logistic Service Providers (LSPs) offering synchromodal transport often seek to consolidate as many containers as possible for the long haul in order to achieve economies of scale. However, consolidating many containers in a single mode (e.g., barge, train) may result in a high number of calls/stops of that mode due to different destinations of containers consolidated. The trade-off between consolidating containers versus postponing containers to form groups with similar destinations is challenging due to the random arrival of freight demand. In this chapter, we design a serious game to raise awareness about this trade-off and to educate on how to optimize it for a target group of LSP practitioners and operations management students. Specifically, we design a game that has a two-fold purpose: (i) to show the impact that scheduling decisions of today have on the performance and the decisions of future days in long-haul single-trip transport and (ii) to demonstrate the use of our 
anticipatory scheduling methods for decision support. Since we already studied the impact of our anticipatory methods to schedule long-haul single-trip transport through extensive numerical experiments in Chapters 2 and 3, we focus in this chapter on designing mechanisms and scenarios for the game and not on extensive experimentation in gaming sessions. We carry out game tests to verify and validate that our design can be used in such sessions.

The game is based on the long-haul single-trip transport scheduling problem introduced in Chapter 2 (Section 2.3.2). In this transport problem, an LSP has to decide whether to assign freights to a high-capacity mode (e.g., barge), assign freights to an expensive alternative (e.g., truck), or postpone the transport of freights, at each decision moment. The goal of the LSP is to minimize its total costs over a planning horizon (e.g., a week), where the costs of the highcapacity mode depend on the destinations visited. The challenge for the player is to find the right postponement, consolidation, and mode-choice decisions, which depend on the game settings. Although this problem was inspired by a Dutch LSP that transports containers between the Eastern part of the country to the Port of Rotterdam, on a daily basis and using a barge, similar scheduling problems can be found in the so-called point-to-point [54], line [54], and corridor [13] network topologies of intermodal transport under the synchromodal characteristics.

The remainder of this chapter is organized as follows. We provide a brief background about serious gaming for freight transport and logistics in Section 7.2. In Section 7.3, we describe our game and its use. In Section 7.4, we report the setup of a gaming session to validate the game and analyze its results. We finalize in Section 7.5 with a discussion and our conclusions.

\subsection{Background}

Serious games are games in which education is the primary goal and entertainment, enjoyment, or fun come as a secondary purpose [62]. In tasks such as aircraft flying and city planning, where real-life experimentation has high costs, risks, or time span, serious games allow participants to experience real-life situations in a safer and faster environment [95]. In the area of complex business processes, serious games have become increasingly important for training and education during the last decades [34]. For example, supply-chain interactions within external and internal actors have been taught in computer simulation games such as the MIT Beer Game [93] and the Fresh Connection [46]. In the fields of freight transport and logistics, simulation games have not been as widely used as in the supply-chain field, even though their potential to understand transport issues and solutions has been recognized [81]. In this section, we briefly study games developed in the freight transport and logistics area with a focus on their purpose, characteristics, and mechanics. Furthermore, we present how our game design can contribute to the area of serious gaming.

In the freight transport and logistics business, serious games have mostly been developed for raising awareness about the interaction among different actors in a transport system [83]. For example, games such as the Rail Cargo Challenge [50] raises awareness about the collaboration among rail operators, terminal operators, and freight forwarders, where some of these roles are competing. Similarly, the distributed barge planning game [60] simulates the interaction between barge and terminal operators and the rail cargo management game [58] simulates the interaction among transporters, clients, and network managers. Games about training a single- 
actor are scarce and focus mostly on passenger or public transport as seen in the review of [81]. For example, Eco-dealers and Waze are two location-based games (using a mobile phone) described in [83] where a single player is trained for the improvement of public and passenger transport respectively. Examples of game that are used to train a single-player that are closely related to ours are Synchromania [11], the follow-up game MasterShipper, and the Modal Manager game [49]. The purpose of these games is to provide the player (who assumes the role of a planner) with insights in how synchromodal characteristics such as mode-free booking can be managed and how they can improve the performance of an intermodal network.

The last game examples show that the enhanced complexity of transport networks requires new thinking of planners that are often not experienced in making advanced decisions. The effectiveness of using games for this target group is increasingly being researched. Our game contributes as a first step towards using a game to effectively teach planners new decision paradigms in a twofold manner. First, we design a game that enables the player to experience anticipatory scheduling using mechanisms based on the optimization algorithms of Chapter 2. Second, we design engaging game components [68] that enable the testing and measuring of its effectiveness towards raising awareness and educating about some of the trade-offs in anticipatory freight scheduling of synchromodal transport.

\subsection{Game Design}

In this section, we describe our single-player freight transport game. Before describing it, we define some terms we use throughout the section. The game master is the person deciding the settings of the game before it starts and the player is the person playing the game. The purpose of the game refers to the objectives of the game master, i.e., to increase awareness and to educate players. The goal of the game refers to the objective of the player within the game: minimize logistical costs. The mechanics refer to the characteristics of the game that influence how well a player achieves his or her goals but which cannot be changed by the game master (i.e., are inherent to the design). The scenarios refer to the settings of the game that can be changed by the game master to achieve the purpose of the game. Scenarios are defined before the game starts and do not change throughout one game.

This section is structured as follows. We begin by describing the problem, goal, and playing mechanics of the game in Section 7.3.1. Following this characterization of the game, we describe the elements of a game scenario, their construction, and their use in Section 7.3.2. Then, in Section 7.3.3, we describe the verification and validation of our game. Finally, in Section 7.3.4, we describe the possible uses of the game for the target audience.

\subsubsection{Mechanics}

The player takes the role of an LSP planner who schedules the transport of containers from the hinterland to a deep-sea port using a daily barge and trucks. The capacity of the barge is limited, but there is an unlimited number of trucks. Every day, the player assigns containers to the barge and trucks of that day. New containers arrive each day according to some probability distribution. The containers arriving are unknown for the player, but are 
pre-defined by the game master. Each container has three characteristics: (i) destination, (ii) release-day, and (iii) time-window length. There are three destinations, or terminals, in the deep-sea port: Red, Green, and Blue. There are two types of release-day: same-day and next-day. Containers with a same-day release can be transported on the day they arrive, while those with next-day release can only be transported from the next day onwards. Time-window lengths can be of one, two, or three days after the release day. Both the daily barge and the trucks take one day to bring a container to its destination, meaning that a timewindow of one means that the container must be transported today, two means tomorrow, and three the day after. All containers must be transported within their time-window.

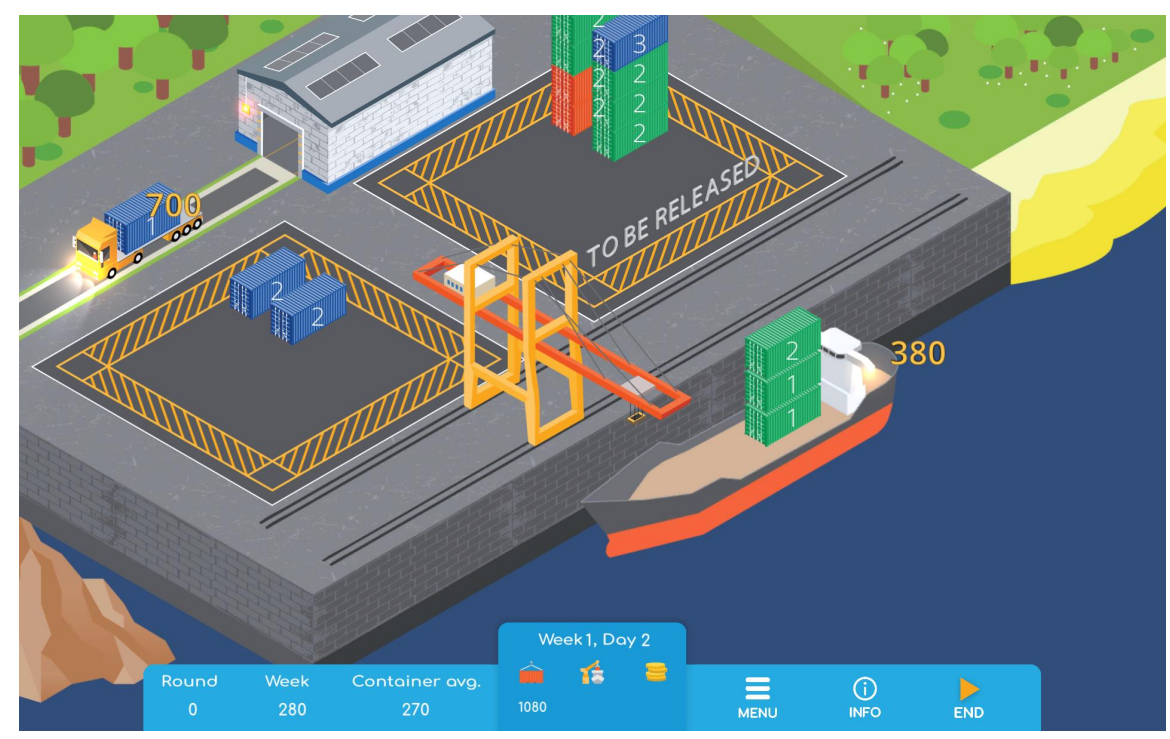

Figure 7.1: Screenshot of the main screen of the game.

To make the gaming experience engaging and fun, we built a digital game of the aforementioned transport situation after an initial board-game prototype. We describe the board-game prototype, and its initial use, in Section 7.3.3, but now focus on the digital game. The digital game has a web-based version that supports the use of different devices (e.g., laptop, tablet, etc.), as well as a stand-alone version for Windows. Both the web-based and stand-alone versions have the same user interface and centralized score keeping capabilities. The main playing screen is shown in Figure 7.1. Containers are colored according to their destination and are located in one of two container yards. The container yard closest to the bottom left corner of the screen contains containers that are released for transport (i.e., can be transported today) and the yard closest to the top right corner contains container that are to be released (i.e., cannot be transported today). Furthermore, containers are labeled with a white number in the middle according to their current time-window (i.e., 1, 2, or 3), 
which decreases as days pass and containers are not transported. To schedule the transport of containers, the player can drag-and-drop containers from the left container yard into the barge or a truck. The player can also drag-and-drop containers out of the barge, or the trucks, back into the left container yard. The schedule of the day (i.e., decision of a player) is finalized and executed when the end button is pressed or when the maximum time for a day's decision has elapsed, which is indicated by a progress bar.

The game is played in turns. Each turn corresponds to a day within a working week of five days. The number of weeks to play is pre-defined by the game master. In each turn, the player must decide which of the released containers (i.e., containers in the left yard) to transport, either by barge or by truck, and which containers to postpone, if any. At the end of each turn (i.e., when the player presses the end button or the maximum time elapses), containers that had a time-window of one and were not transported will be automatically assigned to the trucks. Then, the barge and the trucks depart (animation), and a day report is presented to the player with the costs of his or her decisions, as shown in Figure 7.2. We elaborate about costs later on in this section. In the transition to the next turn, two things happen: (i) containers to be released the next-day (i.e., containers in the right yard) are moved to the left yard and (ii) new containers arrive to the two container yards. It is important to mention that, even though these new containers that arrive are not known to the player, they are predefined by the game master. Furthermore, the game master also pre-defines the containers to appear in both yards at the beginning of the week (i.e., the initial state of the system). The turns continue until the end of the week, where the game "cleans" the containers that were left and assigns cleaning costs to the player. At the end of a week, the player gets a report on his or her costs for each day and the cleanup costs, as shown in Figure 7.3.

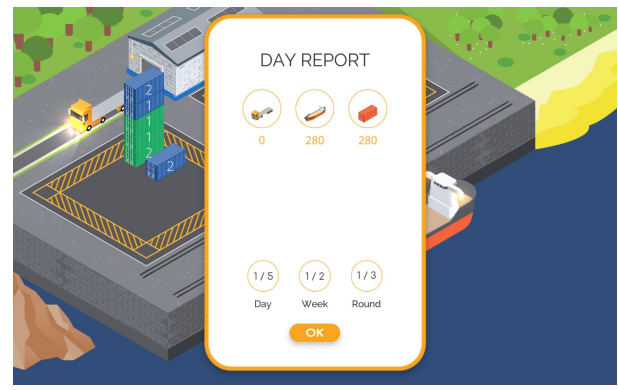

Figure 7.2: Screenshot of the day report.

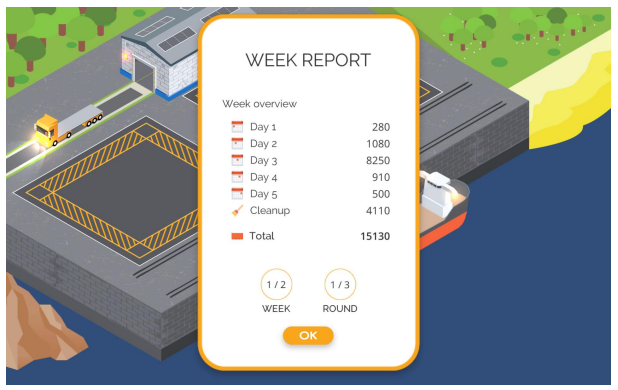

Figure 7.3: Screenshot of the week report.

For each container in the yard of released containers (left of Figure 7.1), the player has three possible decisions: (i) transport by barge today, (ii) transport by truck today, or (iii) postpone its transport to a future day. The first two options result in immediate costs, which are displayed next to the barge or the trucks, whereas the third option does not. However, the third option will influence the decisions on future days and their costs. In the game, all immediate cost information can be accessed at any time by pressing the info button, as shown in Figure 7.4. For the barge, there are two cost components: a setup cost 
and a variable cost. The setup cost, as motivated by the Dutch LSP the game is based on, depends on the combination of destinations visited. Hence, visiting two destinations together in the same barge does not correspond to the sum of visiting each of them separately. The variable cost depends also on the destination, but is added per container consolidated in the barge. For the trucks, there is only a variable cost component that depends on the destination and is incurred per container transported. All of these costs are pre-defined by the game master, as well as the capacity of the barge.

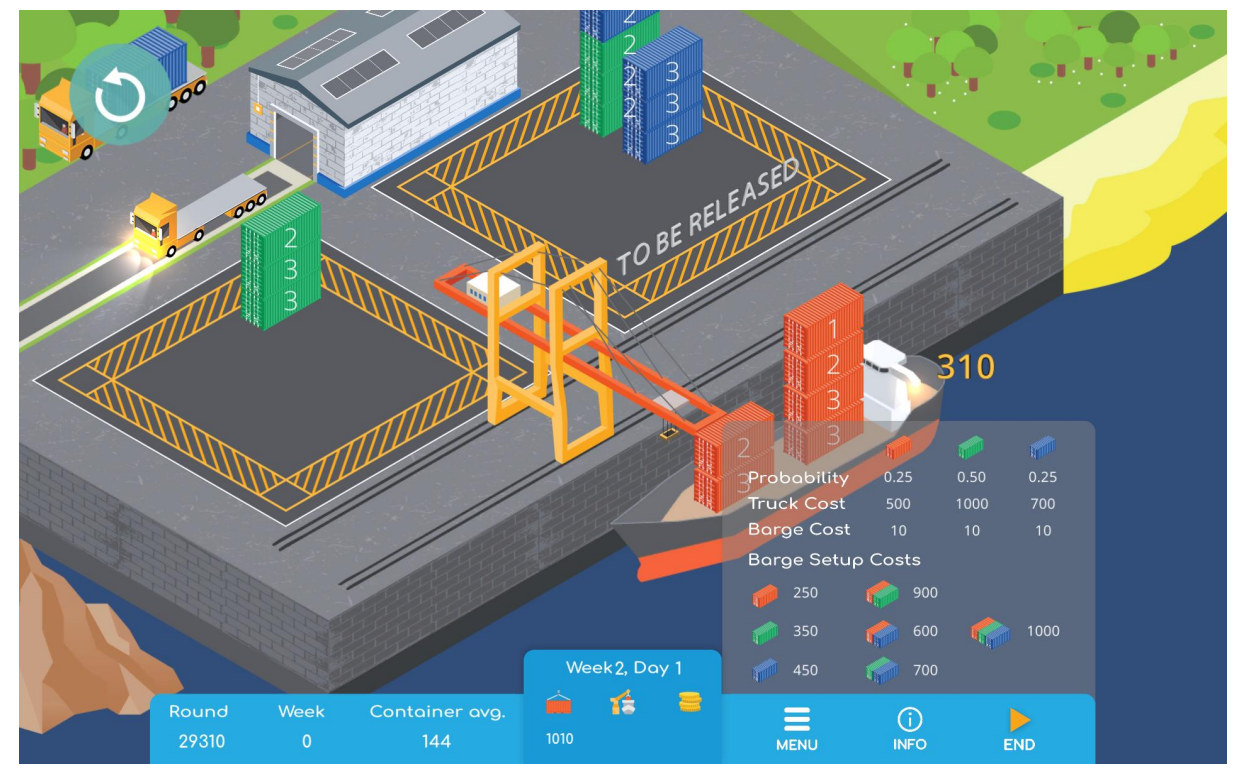

Figure 7.4: Screenshot of the game when the player presses the info button.

The goal of the player is to deliver all containers with the minimum total costs over all weeks. To achieve this, the player can use the decision-specific cost information displayed next to the transport modes or the general cost information displayed when the info button is pressed (see Figure 7.4). Such a situation resembles real-life and current practice, where this information is easily accessible to planners. We define this playing situation as the normal mode of the game. However, a better situation would be when the planner has information about future costs, based on today's decisions and on the available freight information. We define this playing situation as the the support mode of the game. In this mode, estimated future costs/savings of the current decision are displayed in the center tab of the main screen, under the crane icon, as shown in Figure 7.5. In the same tab, the immediate costs of the current decision are displayed under the red container; and their sum is displayed under the coins. This sum supports the player in evaluating the marginal future costs/savings of decisions with different immediate costs, as exemplified when comparing Figures 7.5 and 7.6. 


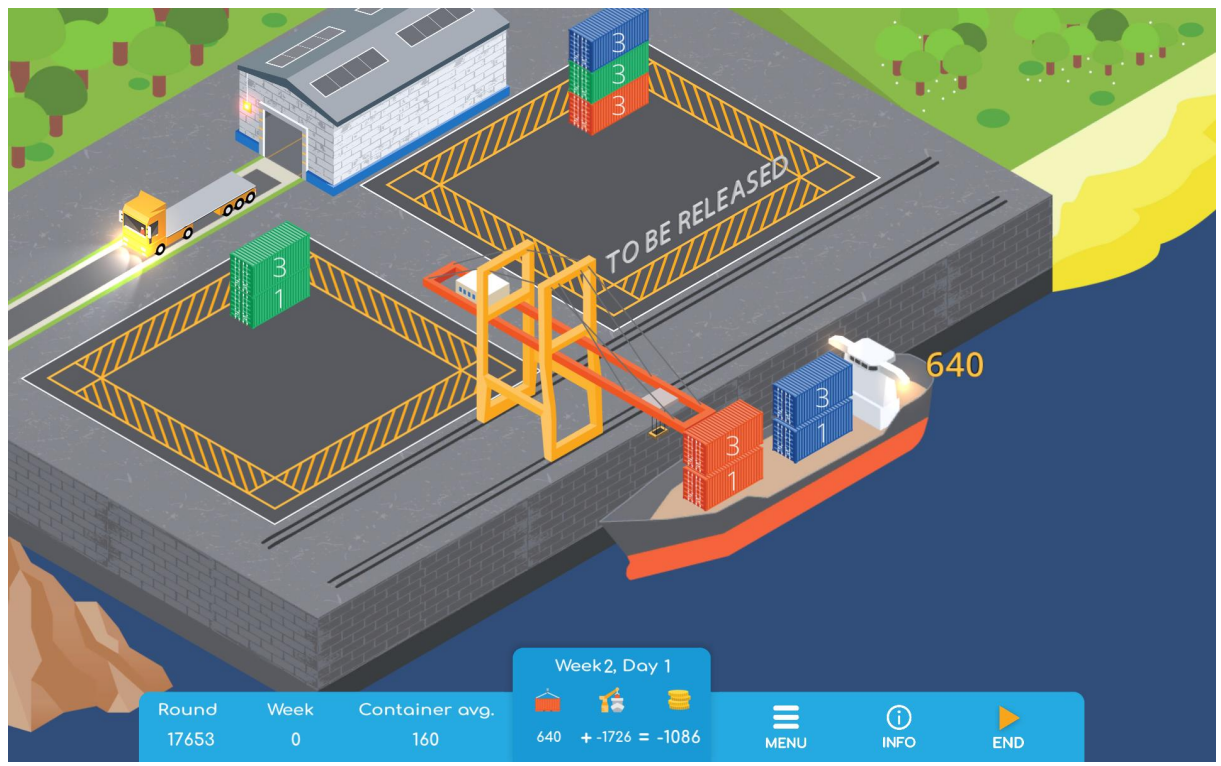

Figure 7.5: Screenshot of the game in support mode, decision A.

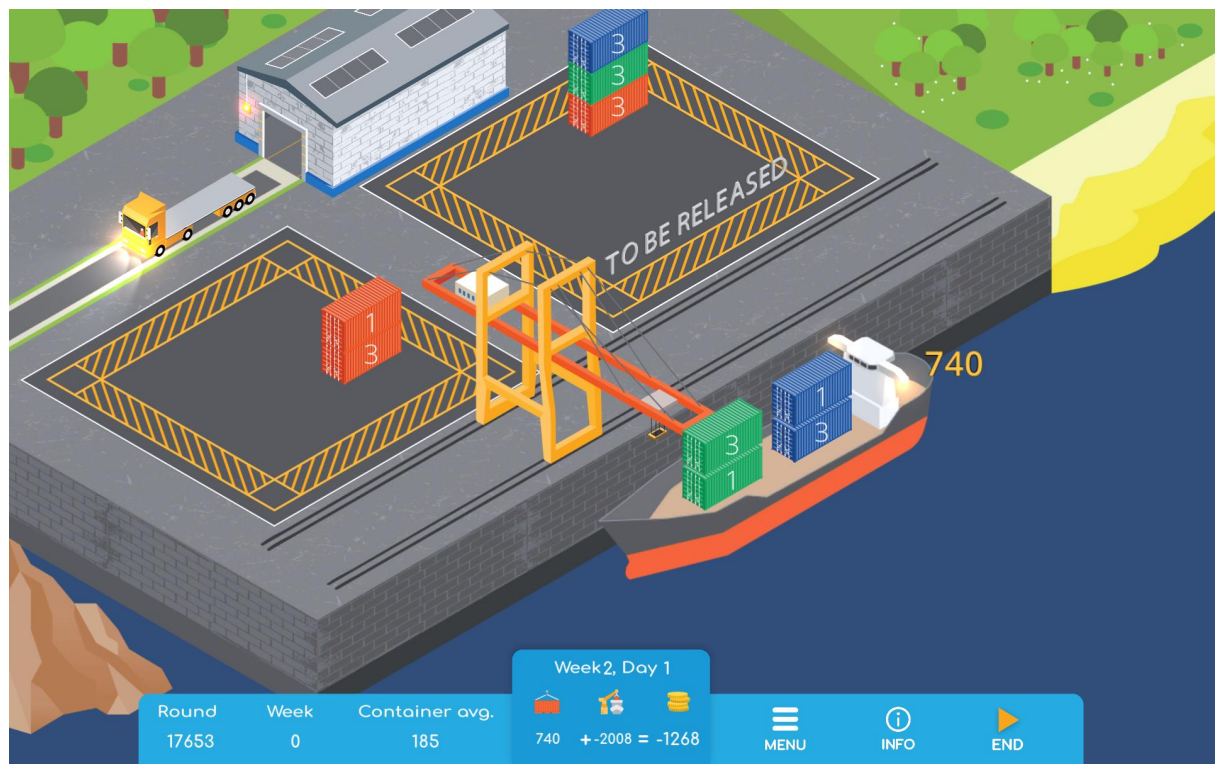

Figure 7.6: Screenshot of the game in support mode, decision $B$. 
In Figures 7.5 and 7.6, we exemplify two decisions that a player can make in the support mode, decision A and B, respectively. Decision A has lower immediate costs than B, but $\mathrm{B}$ has lower estimated future costs (or larger savings given the negative sign). The player can decide whether he or she wants to incur larger costs today with potential savings on future days. Naturally, the decision depends on whether the player expects the estimated future costs to be close to what the future will result in. In the following, we explain how these estimated future costs/savings are calculated.

To estimate the change in future costs $\widehat{C}_{t}(x)$ of a decision $x$ at a day/turn $t$, we use the approximated next-stage costs $\bar{V}_{t}$ defined in Equation (2.14) from Chapter 2 For the basis functions and weights required by this equation, we use the ADP algorithm and Features 3 found in Table 2.2 of Chapter 2. The basis functions are embedded in the game and cannot be changed. The weights, however, can be changed by the game master as we discuss later on in Section 7.3.2. We define $\widehat{C}_{t}(x)=\bar{V}_{t}\left(S_{t}^{x}\right)-\bar{V}_{t}\left(S_{t}^{\emptyset}\right)-c^{u}(x)$, where $S_{t}^{\emptyset}$ is the post-decision state of making no decision, $S_{t}^{x}$ is the post-decision state of making decision $x$, and $c^{u}(x)$ is the reduction in automatic trucking costs due to decision $x$. In other words, the estimated future costs can be interpreted as the costs-to-go resulting from a specific player's decision. For example, decision A in Figure 7.5 has estimated savings of 1726 over not assigning any container to a vehicle, whereas decision B in Figure 7.5 has savings of 2008. In decision $\mathrm{A}, c^{u}(x)=500+700=1200$ is the reduction in automatic trucking costs for transporting one red and blue container with time-window 1 (see costs in Figure 7.4).

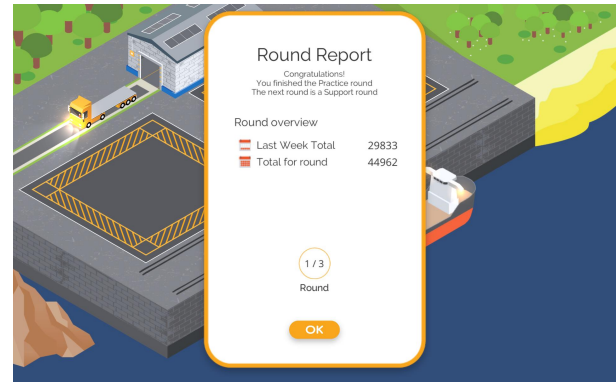

Figure 7.7: Screenshot of the round report.

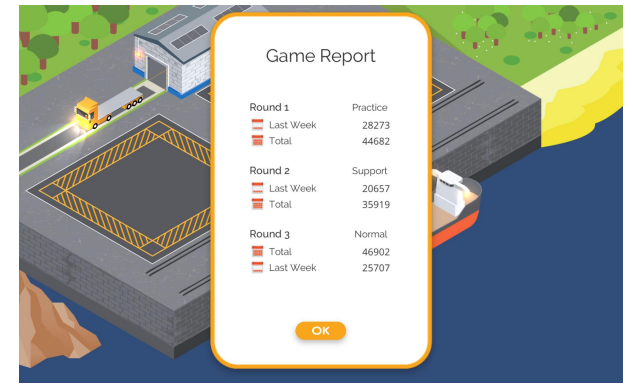

Figure 7.8: Screenshot of the game report.

Since we have two game modes where the player has different levels of support for scheduling the transport of containers, we play the game in rounds. A round consists of a pre-defined number of weeks where a player is in the same mode, i.e., normal or support. In addition to these two modes and round types, we introduce a third option: a practice mode. With a practice mode, the game master is able to control the learning effect present when playing the game, and with these, make better comparisons between the normal and support mode. A game can consist of at most one round of each type, in any sequence, for a maximum of three rounds. At the end of a round, a round report is displayed, as shown in Figure 7.7. The round-report has a special indicator: the performance of the last week of that round. We believe this is a good indicator to compare players, since during the first weeks, 
learning effects of a new type of round may occur (we elaborate more on using the game to compare performance in Section 7.3.4). At the end of the game, a game report is displayed, as shown in Figure 7.8, which includes the aforementioned special indicator for each round.

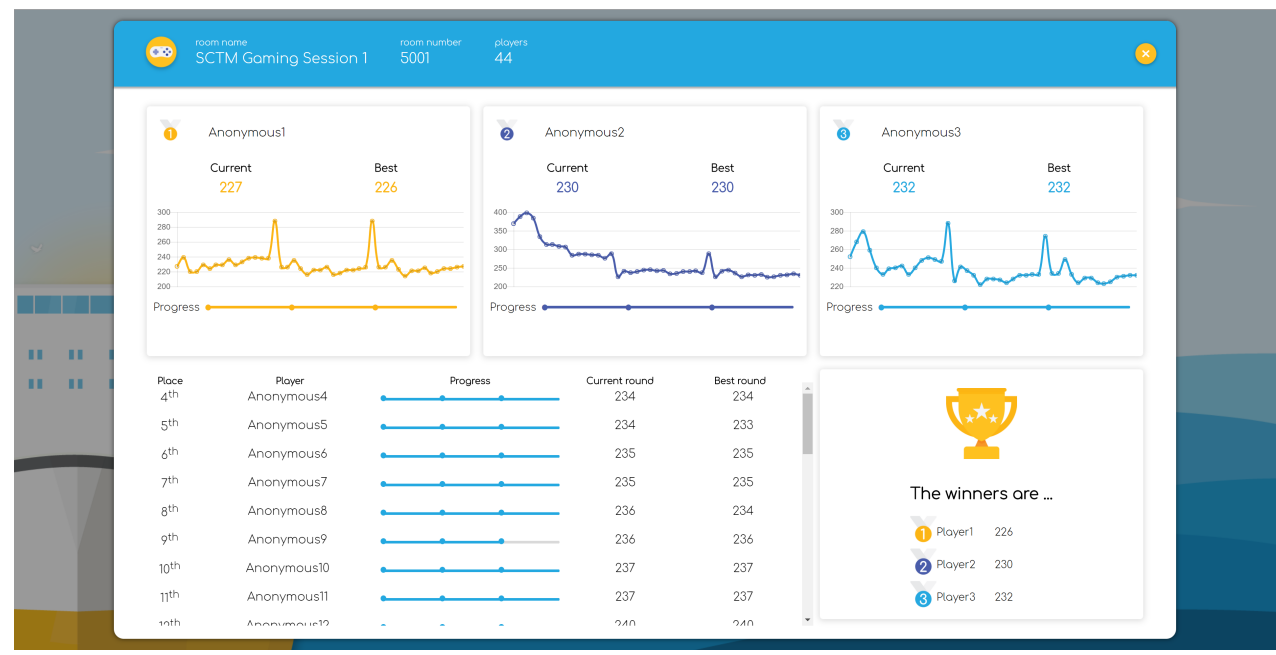

Figure 7.9: Leader-board showing the performance of all players within a given game room.

The game also has a dynamic leader-board, as shown in Figure 7.9, where players can see the real-time costs of all players active in the session. For all players, the average costs per container in the current and best round so far as shown, together with the progress of the player. For the top 3 players, graphs with the average costs per container per day are shown. A final top 3 of players that completely finished the game is shown in the bottom right corner. Before starting a game session, players have to enter a nickname and room-key that determines the web-based leader-board where costs will be posted to. The room-key is a unique identifier for a game session defined by the game master. The game itself posts player costs after every turn ends, but the game master can decide how often updates to the leader-board are shown (e.g., after every turn, after every round, etc.), as shown in Figure 7.9. All costs and decisions, per player and per room-key, are stored centrally, in a web server. The room-key works in a similar fashion to the classroom web-based application Socrative. ${ }^{1}$

The game can be seen as a single-player game with competition elements, i.e., a player's decisions with corresponding results do not depend on other players. Hence, the game can be played by any number of players. To boost competitiveness, players do receive live updates of the other players within the same session through the dynamic leader-board. The playing time depends on the time limit for the daily decision, which is limited to 60 seconds. Hence, the duration for a typical gaming session with 2 game scenarios, each having 3 rounds of 3 weeks, is 90 minutes. The game has a web-based version that supports

\footnotetext{
1 https://www.socrative.com/
} 
the use of different devices (e.g., laptop, tablet, etc.) and platforms.

Table 7.1: Game Settings

\begin{tabular}{ll}
\hline Game Setting & Value(s) \\
\hline Round 1 mode & [Practice, Normal, Support] \\
Round 2 mode & [Practice, Normal, Support, None] \\
Round 3 mode & [Practice, Normal, Support, None] \\
Number weeks per round & {$[1,2,3,4,5]$} \\
Randomize & [Yes,No] \\
Random Seed & Integer number \\
Initial states for Practice mode & $\{$ State ID per week $\}$ \\
Initial states for Normal mode & \{State ID per week $\}$ \\
Initial states for Support mode & $\{$ State ID per week $\}$ \\
Random freights for Practice mode & $\{$ Order ID per day, per week $\}$ \\
Random freights for Normal mode & $\{$ Order ID per day, per week $\}$ \\
Random freights for Support mode & $\{$ Order ID per day, per week $\}$ \\
Barge capacity & {$[6,8,10]$} \\
Barge setup costs & $\{$ Cost per destination combination $\}$ \\
Barge variable costs & $\{$ Cost per destination $\}$ \\
Truck variable costs & CCost per destination $\}$ \\
Basis function weights & $\{$ Number per basis function, per day, per week $\}$ \\
\hline
\end{tabular}

All settings that can be pre-defined by the game master are shown in Table 7.1. The values in square brackets mean that only one of the options between brackets can be chosen. The values in curly brackets mean that several values are needed, one per item described. For example, if there are three weeks per round, then the initial states for each mode require three states from an indexed list that has an identifier (ID). Remind that a state is a collection of containers with known characteristics analogous to the state of the MDP model in Chapter 2. Similar to the states list, there is also an indexed orders list for the random freights for each mode. An order is a collection of containers with known characteristics, as in a state, with the addition of a probability of arrival. We elaborate more on the states and orders, as well as their indexed lists, in the following section describing game scenarios.

The only settings from Table 7.1 not yet explained are the randomize and random seed. The randomize setting is a yes/no setting defining whether the orders that the player will have to transport for all days, weeks, and rounds are pre-defined (in the initial state and random freights settings) by the game master or not. If randomize is set to yes, then the game does not use the pre-defined orders but uses the random seed to randomly select orders from the indexed list, following the probability distribution of orders. With this settings, the game master can determine whether he or she wants absolute control over the game, depending on the purpose and use of it. Later on, in Section 7.3.4, we further elaborate on the randomize option for the game master and the potential uses of our game. We now describe the elements forming a game scenario. 


\subsubsection{Scenarios}

The aforementioned game settings influence the degree of freedom of the player with respect to decisions, costs, and information support. As a consequence, the goal and performance of the player depends on these settings. However, the purpose of the game master towards the player depends not only on these settings, but also on the state and order list used as input. The order list, exemplified in Table 7.2, determines the scheduling situations that the player will face, such as a large number of different containers most of the days or a small number of predictable containers every day. The state list, which contains the same information fields as the order list except for the probability, determines the containers that the player encounters at the beginning of each week. The game settings together with the state and order lists constitute what we define as a game scenario. In the following, we discuss how to build these two tables (i.e., the order list with its corresponding game settings) and exemplify considerations that the game master can have when building them.

Table 7.2: Example of an order list

\begin{tabular}{|c|c|c|c|c|c|c|c|c|c|c|c|c|c|c|c|c|c|c|c|}
\hline \multirow{2}{*}{ ID } & \multirow{2}{*}{$\begin{array}{l}\text { Cumulative } \\
\text { Probability }\end{array}$} & \multicolumn{9}{|c|}{ Same-day release } & \multicolumn{9}{|c|}{ Next-day release } \\
\hline & & $\mathrm{R} 1$ & $\mathrm{R} 2$ & R3 & G1 & G2 & G3 & B1 & B2 & B3 & $\mathrm{R} 2$ & R3 & $\mathrm{R} 4$ & G2 & G3 & G4 & B2 & B4 & B4 \\
\hline 1 & 0.2 & 1 & - & - & 1 & - & - & - & - & - & - & - & - & - & 1 & 1 & - & - & - \\
\hline 2 & 0.5 & - & - & 1 & - & 2 & - & 1 & - & - & - & 2 & - & 2 & - & - & 1 & - & - \\
\hline 3 & 1 & - & 2 & - & - & - & 2 & - & 1 & - & 1 & - & 1 & - & - & - & - & 2 & - \\
\hline
\end{tabular}

In the case of no randomization, all initial states and orders are pre-defined by giving their ID in the lists. The game then selects the corresponding row and retrieves the information about containers. In the case of randomization, the game selects a random initial state from the list for each week following a uniform distribution, and selects an order for each day following the cumulative probability found in the order list. Since the order list is based in the stochastic process describing the arrival of containers and their characteristics, orders do not all necessarily have the same probability. This stochastic process consists of four probability distributions: (i) number of containers per day, (ii) destination per container, (iii) release-day per container, and (iv) time-window length per container, as exemplified in Tables 7.3 and 7.4. Using these probabilities, the game master can build the list of all possible orders and calculate the arrival probability of each order. Orders are analogous to the exogenous information described in Section 2.3.2 from Chapter 2, and their arrival probability can be computed using Equation (2.10) from the same section.

Table 7.3: Example probabilities of container arrivals per day

\begin{tabular}{|c|c|c|c|c|c|}
\hline Containers & Probability & Containers & Probability & Containers & Probability \\
\hline 1 & 0.03 & 5 & 0.19 & 8 & 0.07 \\
\hline 2 & 0.08 & 6 & 0.15 & 9 & 0.04 \\
\hline 3 & 0.14 & 7 & 0.1 & 10 & 0.02 \\
\hline 4 & 0.18 & & & & \\
\hline
\end{tabular}


Table 7.4: Example probabilities of characteristics per container

\begin{tabular}{cccccccc}
\hline Destination & Probability & & Release-day & Probability & & Time-window & Probability \\
\cline { 1 - 2 } Red & 0.2 & & Same-day & 0.4 & & Today & 0.4 \\
Green & 0.3 & & Next-day & 0.6 & & Tomorrow & 0.5 \\
Blue & 0.5 & & & & Day-after tomorrow & 0.6 \\
\hline
\end{tabular}

In addition to defining the order lists, the four probability distributions are used to determine the weights of the basis functions that are used to estimate future costs in the support mode of the game. To determine these weights, the game master can run the ADP algorithm in the same way that was done for the numerical experiments in Section 2.5 from Chapter 2. The ADP algorithm also requires the initial state for each week and the capacity of the barge. It is important to note that the selection of the initial state can determine the "suitability" of anticipatory scheduling achieving good results in the game. As an aid, the game master can use the results of the extensive numerical experiments in Chapter 3 to determine those starting conditions. In the case of the randomized game scenario, where orders are randomly selected from the list rather than pre-determined by the game master, the weights in the support round must be calculated using a variant of the ADP algorithm in Chapter 2. The variant must be designed with an infinite horizon such that the weights learned result in a stationary policy that minimizes the expected costs over all possible states and stages. An example of how an ADP algorithm with infinite horizon can be designed for this problem can be found in the freight consolidation problem from Section 5.2 in [85].

With different game scenarios, the game master can represent a large number of LSP characteristics, such as unbalanced destinations, pre-announced freights, tight time-windows, etc. Furthermore, the game master can forecast for which initial states he or she can best achieved the purpose of showing the benefits of anticipatory scheduling over other benchmarks (or the lack thereof) using the results of Chapter 3, or the experimental setup in case of new LSP characteristics. The flexibility in game scenarios allows the game master to decide on which aspects/benefits of anticipatory scheduling to educate on. From the key elements of learning while playing described by [50], our game scenarios have:

Active participation In both the normal and support modes of any game scenario, the player has complete control of the outcome of the game. This control plays a crucial role in the support mode of a game scenario where anticipatory scheduling does not pay off, since following the cost-to-go estimate may not be the best strategy. Furthermore, the dynamic leader-board can increase the involvement of a player in his or her decisions when, for example, the game master shows live changing results after each turn.

Challenge Game scenarios can vary with increasing difficulty, giving the player who knows the game something to look forward to. Furthermore, leader-boards per game scenario allow the game master to renew the challenge when new players are to be educated.

Interaction Players can compare their scores for the same game and discuss on how the strategies that they follow lead to a good or bad performance. Furthermore, the best ranked players in the leader-board can describe how the lowest costs for that scenario can be achieved, and what anticipatory elements they found the most important. 
Our game has two main purposes: to raise awareness about the trade-offs in anticipatory scheduling of synchromodal transport and to educate on how to optimize them. For achieving both objectives, it is important to verify the game mechanisms and validate the idea of game scenarios. In the following section, we explain how we verified that our web-based simulation game worked and that the use of game scenarios is ideal for players to identify key aspects of the game's purpose. Afterwards, we give examples of scenarios for different uses of the game.

\subsubsection{Verification and Validation}

Before the implementation of our ideas in a web-based game, we developed a board-game prototype of our design. Several tests were carried out with $\mathrm{PhD}$ students of industrial engineering and business information systems, and several adaptations to the concept were made, until we came to the version shown in Figure 7.10. Some of the most common comments from players were about the availability on information of the game, such as transport costs, arrival probabilities, etc. The more keen players asked for information of the distribution of destinations and time-windows, such that they could forecast what containers would arrive. Hence, in this preliminary design, we had a game mode where a forecast about container arrivals could be made. In the digital version, this was changed towards the use of a forecast about future costs rather than container themselves, since we observed players were more keen on basing their decisions on costs rather type of containers transported.

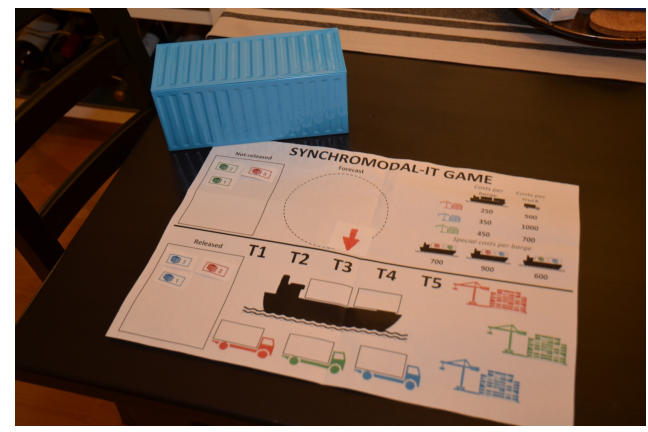

Figure 7.10: Initial game prototype: paper version.

In the board-game version, the game master had to manually move out containers that were transported, enter decisions in a spreadsheet model to compute costs, and add new containers for the next day. However, in the digital version, all of these steps are carried out by the game code. To simplify the code of the web-based game, we designed several aspects in a way that the algorithms and formulas from Chapter 2 could be separated from the game's logic. For example, the state list, order list (with its probabilities), and basis function weights are all computed outside the game and used as input read from a file. Nevertheless, aspects such as the evolution of the system through time (i.e., state transition) and the computation of cost-to-go (based on the basis functions and input weights) needed 
to be inside the game code. For verifying that the game code worked as expected, and that the simulation and measurements actually did what we intended to, we followed two steps:

1. Manual testing We developed a spreadsheet model of the game, where all input files could be generated, where decisions could be entered, where new orders and transitions could be easily seen, and where immediate and future costs could be computed. Using this spreadsheet model, we could determine the outcomes of the game up front. With this spreadsheet model on hand, we played the game multiple times, varying some settings or decisions each time. We then compare the outcomes of the spreadsheet model to what was shown on screen to test whether the code was doing what was expected.

2. Extreme playing We played the game using extreme scenarios (e.g., many containers) and with unorthodox scheduling decisions. For each day and week of all round types, we carefully looked at aesthetic and quantitative elements (e.g., number of containers of a type) shown on the screen.

3. Heuristics comparisons Following the benchmark heuristics that were used in the numerical experiments of previous chapters, we entered decisions in the digital game with the randomized setting. Since we had code to compute the expected (average) results of using the heuristics, we compared their results to our game to test whether the results at the end of a week were similar to those computed outside the game.

Naturally, these steps revealed bugs and glitches in the game. Together with the game programmers $^{2}$, we iterated over these steps until a satisfactory game version was achieved. For validating the game, various gaming sessions were organized with $\mathrm{PhD}$ students of our industrial engineering and business information systems department, as well as researchers from other Dutch universities. In these gaming sessions, we observed that the main differences among players occurred in:

- Information used when scheduling, both in the normal and support mode. Players that achieved the lowest costs tended to press the info button often to check which combination of destinations were cheaper for the barge. Other players simply used dragand-drop of different containers (i.e., trial and error) until the minimum costs were found.

- Identification of container arrival patterns. Players that achieved the lowest costs usually identified which color of container arrived the most and made decisions with this in mind. Some even claimed to postpone containers knowing that in the future they can do a full barge of only that color.

- Biased strategy towards a personal preference. Some players observed that trucks where significantly more expensive and followed a simple strategy: to send everything they could by barge. This was not the best strategy for the entire horizon of the game, but players identified it as easy to follow (e.g., not thinking too much) and not performing that bad.

With these final steps, our game design is ready to be deployed to the target audience: LSP practitioners and operations management students. Besides the target groups, partici-

\footnotetext{
${ }^{2}$ Pineapple Studios www.pineapplestudios.nl
} 
pants in the game sessions pointed out that the game is fun to play and accessible for people of all ages who may play it for other purposes. As an example, they mentioned playing the game during the open days our university, at bachelors level with participants from high-school, and at masters level with participants from other universities and countries. Such gaming sessions can make the potential new students enthusiastic about technical studies. In the following section, we discuss on how our game can be used for different purposes and what considerations need to be taken into account.

\subsubsection{Game Use}

Even though our game is a single-player one, the leader-board competition element and the inherent didactic aspects make our game ideal for use in sessions with a group of players playing simultaneously and receiving group feedback during, and after, the rounds are played. To ease the organization of such gaming sessions, our game runs on multiple platforms, as shown in Figure 7.11. In the following paragraphs, we discuss three possible uses of our game in such group gaming sessions. Furthermore, we exemplify game scenarios that can be used in gaming sessions for different purposes.
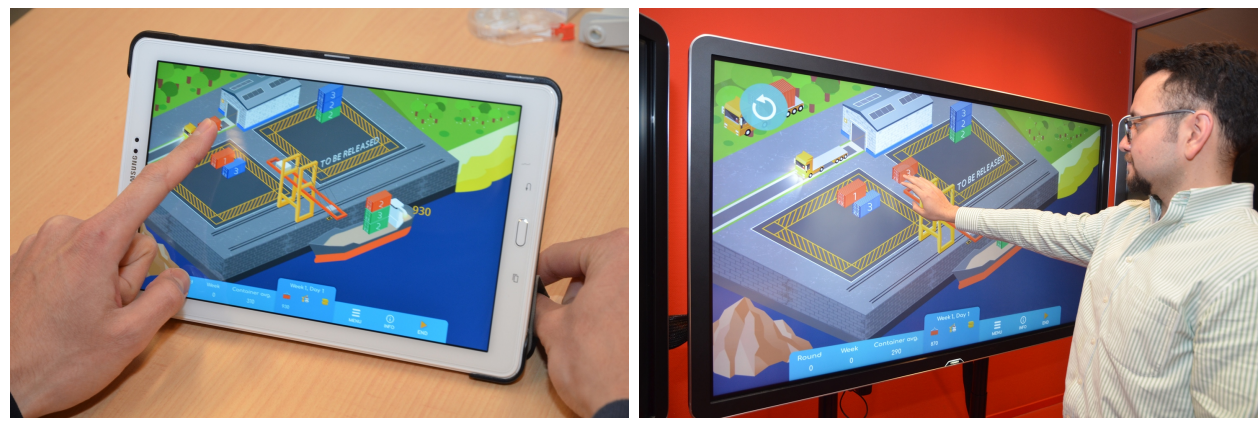

Figure 7.11: Game being played in a tablet and an interactive display.

Related to the setting to randomize the game, we envision three uses of our game, i.e. three types of gaming sessions:

1. Pre-defined research scenarios. In this type of gaming session, the game master pre-defines all initial states and orders (i.e., the randomize setting is set to no) and calculates the performance (i.e., logistical costs) that can be obtained for each round, following different scheduling policies and using the spreadsheet model. The main purpose of such session is to study the increase in awareness or the learning of a scheduling concept. Consider, for example, a gaming session with operations management students where the purpose is to educate on the optimal balance between transporting containers in today's barge and postponing their transport. The game master can design scenarios where postponing is a good or a bad decision, teach students on which problem characteristics make the postponing decision a good or a bad one, and then test the students on an 
unknown scenario, but known to the game master, whether or not the students learned the concept. Consider, in another example, a gaming session with LSP planners where the purpose is to measure awareness of the benefits of using anticipatory scheduling support. The game master can, for example, first do a practice round with everyone and then divide the group into two parts: one playing a normal round followed by a support round, and the other playing a support round followed by a normal round. Naturally, the division is known for the game master, but for the players they only know they are playing three rounds. Furthermore, since the game master has control over all orders, the support round can look the same for every player independent on whether he or she played it before or after the normal round. In such case, the game master can measure cost differences between the normal and support mode among players with a high degree of repeatability and reliability.

2. Pre-defined competitive scenarios. In this type of gaming session, the game master pre-defines initial states and orders as in the research scenario, or sets the randomize setting to yes but uses a known random seed such that random initial states and orders are replicable. The main purpose of such session is to enable players to compare themselves against other players and initiate discussions among them about their performance. Consider, for example, a gaming session with logistics practitioners where the purpose is to challenge the traditional paradigm of consolidating as much freight as possible. The game master can define scenarios where sending a full barge does not lead to the optimal costs and let the players play in the normal mode. If at least one of the players achieves the optimal costs (which are known to the game master), the game master can start the discussion by showing the leader-board and the decisions made by the best player. Naturally, there is the risk of none of the players following the optimal strategy. If this happens, the game master can let the players play a support round, suggesting that if they follow the support, they can improve their performance. After such support round, another discussion could be started, where practitioners can share their experiences and have a long-lasting impression on their paradigm.

3. Randomized scenarios. In this type of gaming session, the game master does not pre-define the orders used in the game (i.e, the randomize setting is set to yes). The selection of a random seed can be done by the player or automatically assigned by the game itself. In both cases of the random seed, the purpose of this type of gaming session is entertainment (playing just for fun). During our validation tests, some of the players commented that they could play our game on their mobile phones for fun if an "all-time best scores" would be kept. In such example, the game master can allow players to choose a random seed, such that two friends could compete against each other knowing they faced the same orders, and assign make of the random seed a room-key (i.e., unique identifier for a specific leader-board) such that the best player of the two friends would be publicly ranked higher. Such a gaming session is enabled by the multi-platform characteristic of our game, which stores all results on a centralized web server.

For the players, the aforementioned types of gaming sessions are accessed differently. The first two types (i.e., the pre-defined scenarios) are accessed by entering a nickname and a room-key. As mentioned earlier, the room-key is a unique identifier defined by the game master, which determines the game scenario and defines the leader-board where results will 
be stored. The last type, which is for entertainment, the player has one additional option. The player can select whether to enter a room-key (e.g., to compete against a friend) or to select from a drop-down menu a game scenario with certain characteristics (e.g., high container arrival, large barge costs). In the latter, a random seed will be assigned by the game itself (within a finite set) for that game scenario and will be used as room-key for the leader-board. This will be displayed to the player, such that it can be shared to other players for playing the same game scenario under the same initial state and order arrivals.

\subsection{Game Test}

As a preliminary test of our game, we organized a gaming session in a classroom with 43 master students of Industrial Engineering and Management at the University of Twente. No demographic information was recorded or used to select the students. The game was played during a lecture hour, in a normal lecture room, as shown in Figure 7.12. The goal of this session was two-fold: (i) to test and validate the game with many simultaneous users, and (ii) to explore whether the game (through its scenarios) can achieve its purpose with a target audience (see Section 7.3.4). In this section, we describe how we performed the test and the results we obtained. First, we motivate the game scenarios used for the test and present their settings in Section 7.4.1. Next, we show the results of the players within the game and present our observations during the gaming session in Section 7.4.2.

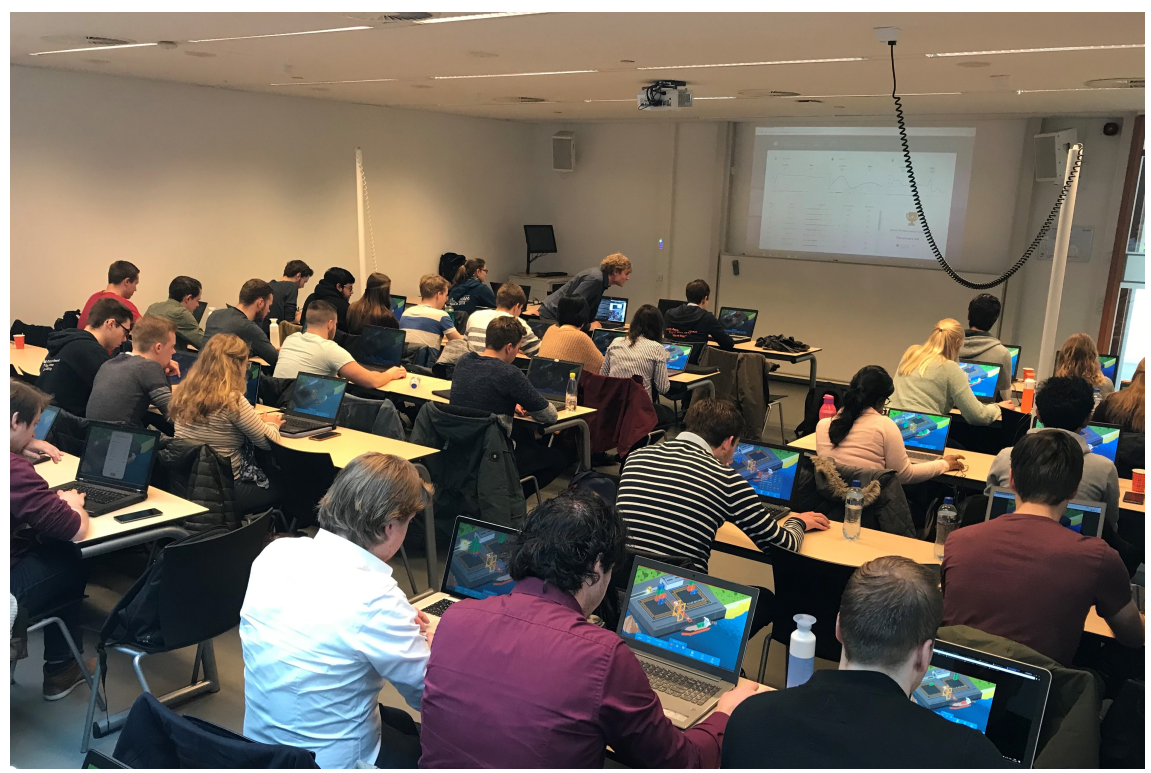

Figure 7.12: Students during the test gaming session. 


\subsubsection{Test Scenarios}

We designed two scenarios that have the same container arrival process but differ in their cost setup. We elaborate on the container arrival process later on in this section, and now focus on the cost setup. The different cost setups represented different LSP characteristics (e.g., an LSP owning a barge and another LSP hiring a barge) and influence the trade-offs a players faces with respect to postponement, consolidation, and mode choice.

The costs for the two scenarios, which we denote as Scenario A and Scenario B, differed in the variable costs of the barge, as shown in Table 7.5. In Scenario A, transporting containers using the barge was always cheaper than transporting them using trucks, for any number of containers and any combination of destinations. The anticipatory trade-off, in this scenario, was mostly due to the combination of destinations visited by the barge and the destinations left for the truck. For example, transporting 1 red and 1 green container in the barge was 300 cheaper than by truck; whereas transporting 1 green and 1 blue container was 660 cheaper. In Scenario B, transporting the containers using the barge was only cheaper above a certain threshold that depended on the combination of destinations and the number of containers per destination. Following the previous example, the red and green combination in a barge was cheaper than the truck when the barge had at least 6 containers (with a majority of green containers); whereas the green and blue combination in a barge was cheaper than the truck when the barge had at least 4 containers (with a majority of green containers).

Table 7.5: Cost setup

\begin{tabular}{lccccccc}
\hline \multirow{2}{*}{ Destination } & \multicolumn{3}{c}{ Scenario A } & & \multicolumn{3}{c}{ Scenario B } \\
\cline { 2 - 4 } \cline { 7 - 9 } & $\begin{array}{c}\text { Barge } \\
\text { setup }\end{array}$ & $\begin{array}{c}\text { Barge } \\
\text { variable }\end{array}$ & $\begin{array}{c}\text { Truck } \\
\text { variable }\end{array}$ & & $\begin{array}{c}\text { Barge } \\
\text { setup }\end{array}$ & $\begin{array}{c}\text { Barge } \\
\text { variable }\end{array}$ & $\begin{array}{c}\text { Truck } \\
\text { variable }\end{array}$ \\
\hline R & 250 & 100 & 500 & & 250 & 400 & 500 \\
G & 350 & 200 & 1000 & & 350 & 800 & 1000 \\
B & 450 & 140 & 700 & & 450 & 560 & 700 \\
R-G & 900 & - & - & & 900 & - & - \\
R-B & 600 & - & - & & 600 & - & - \\
G-B & 700 & - & - & & 700 & - & - \\
R-G-B & 1000 & - & - & & 1000 & - & - \\
\hline
\end{tabular}

The container arrival process was stochastic and behaved according to the following empirical distributions. Every day, 6 containers arrived with probability $0.2,7$ containers with probability 0.5 , and 8 containers with probability 0.3 . The characteristics of each container were distributed as shown in Table 7.6. We defined these characteristics following the results of our experiments in Chapter 3 where anticipatory scheduling payed off. The capacity of the barge was set at 8 containers.

The number of weeks per round was set at 3. All types of rounds (i.e., practice, normal, and support) were played in both scenarios. The initial states of each week of each round were defined to give the player different initial conditions, as shown in Table 7.7. The orders that arrived each day (i.e., set of new containers that were added after a decision), were defiend 
Table 7.6: Probabilities of characteristics per container

\begin{tabular}{cccccccc}
\hline Destination & Probability & & Release-day & Probability & & Time-window & Probability \\
\cline { 1 - 2 } Red $(\mathrm{R})$ & 0.25 & & Same-day & 0.3 & & Today & 0.1 \\
Green $(\mathrm{G})$ & 0.5 & & Next-day & 0.7 & & Tomorrow & 0.1 \\
Blue $(\mathrm{B})$ & 0.25 & & & & & Day-after tomorrow & 0.8 \\
\hline
\end{tabular}

using the 24 container combinations with the largest probability. These orders are shown in Table 7.8, where each group of 4 consecutive orders correspond to the week of a round. The initial states and orders for the normal and support rounds were chosen to be the same, such that the difference in performance of a single player between the normal and support round would be due only to his or her decisions and not to the variability of the container arrivals.

Table 7.7: Initial states of the practice round (1-3) and normal/support rounds (4-6)

\begin{tabular}{|c|c|c|c|c|c|c|c|c|c|c|c|c|c|c|c|c|c|c|}
\hline \multirow{2}{*}{ ID } & \multicolumn{9}{|c|}{ Same-day release } & \multicolumn{9}{|c|}{ Next-day release } \\
\hline & $\mathrm{R} 1$ & $\mathrm{R} 2$ & R3 & G1 & G2 & G3 & B1 & $\mathrm{B} 2$ & B3 & $\mathrm{R} 2$ & R3 & $\mathrm{R} 4$ & G2 & G3 & G4 & $\mathrm{B} 2$ & B4 & B4 \\
\hline 1 & - & 1 & 1 & - & 1 & 2 & - & 1 & 2 & - & 2 & 1 & - & 2 & 2 & - & 1 & 2 \\
\hline 2 & 3 & 1 & - & - & - & - & - & - & 2 & 1 & - & - & - & 2 & 2 & - & - & - \\
\hline 3 & 1 & - & 1 & 2 & 1 & - & 1 & 1 & - & 1 & - & 1 & - & 1 & 2 & - & 1 & - \\
\hline 4 & - & - & 1 & 3 & - & - & 2 & 2 & - & - & - & - & - & 1 & 2 & - & - & - \\
\hline 5 & 3 & 2 & - & - & - & - & - & 3 & 1 & - & - & - & - & - & 2 & 2 & - & - \\
\hline 6 & 1 & - & - & - & - & - & - & 1 & - & - & - & 3 & - & - & - & 1 & 1 & - \\
\hline
\end{tabular}

We defined 2 room-keys per scenario, to vary the sequence of the rounds as follows. Room-key 5001 and 5003 corresponds to a sequence of practice, normal, and support rounds for Scenario A and Scenario B, respectively. Room-key 5002 and 5004 corresponds to a sequence of practice, support, and normal rounds for Scenario A and Scenario B, respectively. With the change in sequence of rounds, we can explore the differences in performance in normal rounds when players have had support before (i.e., learning effects). The 43 students were randomly divided into two groups, where each group played the two room-keys aforementioned. The first room-key, corresponding to Scenario A, was played during the lecture hour. The second room-key, corresponding to Scenario B, was left as homework. The time limit for the daily decision was set to 60 seconds. Hence, the duration for each game scenario (room-key) was at most 45 minutes. The game was played on its web-based version, on the personal laptops of the students.

\subsubsection{Results and Observations}

In this section, we present and discuss the performance of the players within the game. The performance (i.e., cost) data of the players was retrieved from the web-server database and cleaned for duplicate and incomplete records. Furthermore, we provide qualitative observations about the gaming session and some of the remarks we received from the students. 
Table 7.8: Order list of the practice round (1-12) and normal/support rounds (13-24)

\begin{tabular}{|c|c|c|c|c|c|c|c|c|c|c|c|c|c|c|c|c|c|c|}
\hline \multirow{2}{*}{ ID } & \multicolumn{9}{|c|}{ Same-day release } & \multicolumn{9}{|c|}{ Next-day release } \\
\hline & R1 & $\mathrm{R} 2$ & R3 & G1 & G2 & G3 & B1 & B2 & B3 & $\mathrm{R} 2$ & R3 & $\mathrm{R} 4$ & G2 & G3 & G4 & B2 & B4 & B4 \\
\hline 1 & - & - & - & - & - & 2 & - & - & 1 & - & - & 4 & - & - & - & - & - & - \\
\hline 2 & - & - & - & - & - & 1 & - & - & 2 & - & - & 4 & - & - & - & - & - & - \\
\hline 3 & - & - & - & - & - & 2 & - & - & 1 & - & - & 3 & - & - & - & - & - & - \\
\hline 4 & - & - & - & - & - & - & - & - & 2 & - & - & 3 & - & - & - & - & - & 1 \\
\hline 5 & - & - & 1 & - & - & 1 & - & - & - & - & - & 3 & - & - & - & - & - & 1 \\
\hline 6 & - & - & - & - & - & - & - & - & 3 & - & - & 3 & - & - & - & - & - & 1 \\
\hline 7 & - & - & - & - & - & 1 & - & - & 1 & - & - & 4 & - & - & - & - & - & 2 \\
\hline 8 & - & - & - & - & - & 1 & - & - & - & - & 1 & 3 & - & - & 1 & - & - & 1 \\
\hline 9 & - & - & - & - & - & 1 & - & - & - & 1 & - & 3 & - & - & - & - & - & 2 \\
\hline 1- & - & - & - & - & - & - & - & - & 2 & - & - & 2 & - & - & 1 & - & - & 2 \\
\hline 11 & - & - & - & - & - & 1 & - & - & 1 & - & - & 1 & - & - & 1 & - & - & 3 \\
\hline 12 & - & - & - & - & - & - & - & - & 3 & - & - & 2 & - & - & - & - & - & 1 \\
\hline 13 & - & - & - & - & - & 1 & - & - & 1 & - & - & 2 & - & - & 1 & - & - & 2 \\
\hline 14 & - & - & - & - & - & 1 & - & - & 1 & - & - & 2 & - & - & - & - & - & 2 \\
\hline 15 & - & - & - & - & - & 1 & - & - & 1 & - & - & 2 & - & - & - & - & - & 3 \\
\hline 16 & - & - & - & - & - & 1 & - & - & 2 & 1 & - & 2 & - & - & - & - & - & 1 \\
\hline 17 & - & - & - & - & - & 2 & - & - & - & - & - & 2 & - & - & - & - & - & 3 \\
\hline 18 & - & - & 1 & - & - & 2 & - & - & 1 & - & - & 3 & - & - & - & - & - & 1 \\
\hline 19 & - & - & 1 & - & - & 2 & - & - & 1 & - & - & 2 & - & - & 1 & - & - & - \\
\hline $2-$ & - & - & 1 & - & - & 1 & - & - & 1 & - & - & 1 & - & - & - & - & - & 2 \\
\hline 21 & - & - & - & - & - & - & - & - & - & - & - & 5 & - & - & 1 & - & - & 1 \\
\hline 22 & - & - & 1 & - & - & - & - & - & 2 & - & - & 4 & - & - & - & - & - & - \\
\hline 23 & - & - & 1 & - & - & - & - & - & 1 & - & - & 5 & - & - & - & - & - & - \\
\hline 24 & - & - & - & - & - & 1 & 1 & - & 1 & - & - & 3 & - & - & - & - & - & 1 \\
\hline
\end{tabular}

The overall results for Scenario A and Scenario B are shown in Tables 7.9 and 7.10, respectively. In Scenario A, we observe that, on average, players performed slightly better with support, with most of the cost savings resulting from the last week. Particularly, support improves the performance more in Room 5001 than in 5002. A reason for the lower difference between the normal and support rounds of players in room 5002 could be that insights from the support round were used in the normal round, since it comes afterwards. We discuss this later on. In Scenario B, similar observations as in Scenario A arise. However, players in room 5004 now have larger improvements with the support than players in room 5003. A reason for this larger difference in room 5004 could be that the interpretation of the support (i.e., information on future costs or savings) is more complex due to the additional trade-off in mode choice, and hence cannot be used later on for the normal round.

In addition to the comparison of costs across rounds, we also compare the costs of players against the use of the ADP policy and the benchmark heuristic from Chapter 2. Remind that, in the support round, the weights of the ADP policy are used to give the player an estimate of his or her future costs. However, the player must still choose which containers to transport such that look-ahead costs (i.e., current + future) are minimized. Given the time limit for a decision, finding the right containers is challenging and the player may not always find them on time (i.e., follow the ADP policy). We also compare with the benchmark heuristic, which is myopic in nature, to test whether players were, to some extent, scheduling in an anticipatory 
Table 7.9: Average costs in Scenario A

\begin{tabular}{cccccccc}
\hline \multirow{2}{*}{ Room } & \multicolumn{3}{c}{ Last week } & & \multicolumn{3}{c}{ Total } \\
\cline { 2 - 4 } \cline { 7 - 8 } & Practice & Normal & Support & & Practice & Normal & Support \\
\hline 5001 & 11,084 & 8,897 & 8,688 & & 32,218 & 26,637 & 26,162 \\
5002 & 10,965 & 8,470 & 8,483 & & 31,374 & 25,938 & 25,999 \\
Average & 11,025 & 8,684 & 8,585 & & 31,796 & 26,287 & 26,081 \\
\hline
\end{tabular}

Table 7.10: Average costs in Scenario B

\begin{tabular}{cccccccc}
\hline \multirow{2}{*}{ Room } & \multicolumn{3}{c}{ Last week } & & \multicolumn{3}{c}{ Total } \\
\cline { 2 - 4 } \cline { 7 - 8 } & Practice & Normal & Support & & Practice & Normal & Support \\
\hline 5003 & 27,166 & 24,370 & 24,314 & & 81,838 & 72,843 & 72,836 \\
5004 & 27,215 & 24,416 & 24,067 & & 82,135 & 72,828 & 72,137 \\
Average & 27,190 & 24,393 & 24,191 & & 81,987 & 72,835 & 72,487 \\
\hline
\end{tabular}

manner. In Figure 7.13, we show the average percentage savings with respect to using the benchmark heuristic. Two interesting observations arise. First, players in Scenario A (Rooms 5001 and 5002) are doing better with support but not achieving the maximum savings they could achieve (i.e., savings of the ADP policy). Second, in the last week of Room 5004, savings of players in the support round were larger than with ADP. Naturally, the ADP policy minimizes the total costs based on the expected realizations of stochastic demand. Hence, for a specific week, better policies may exist. In the total savings of Room 5004, which are over three weeks, we observe players in the support round achieve similar savings as the ADP policy.

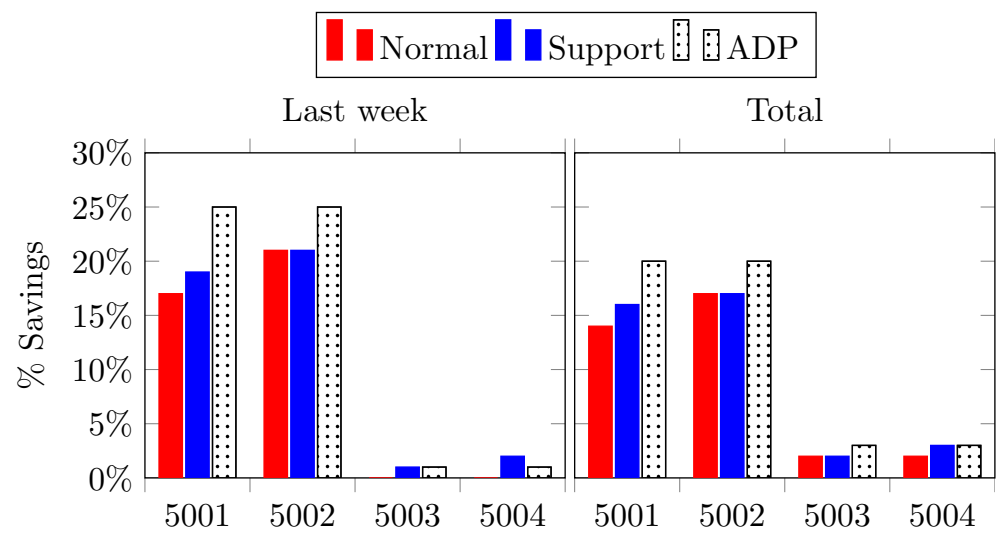

Figure 7.13: Average costs savings with respect to the benchmark 


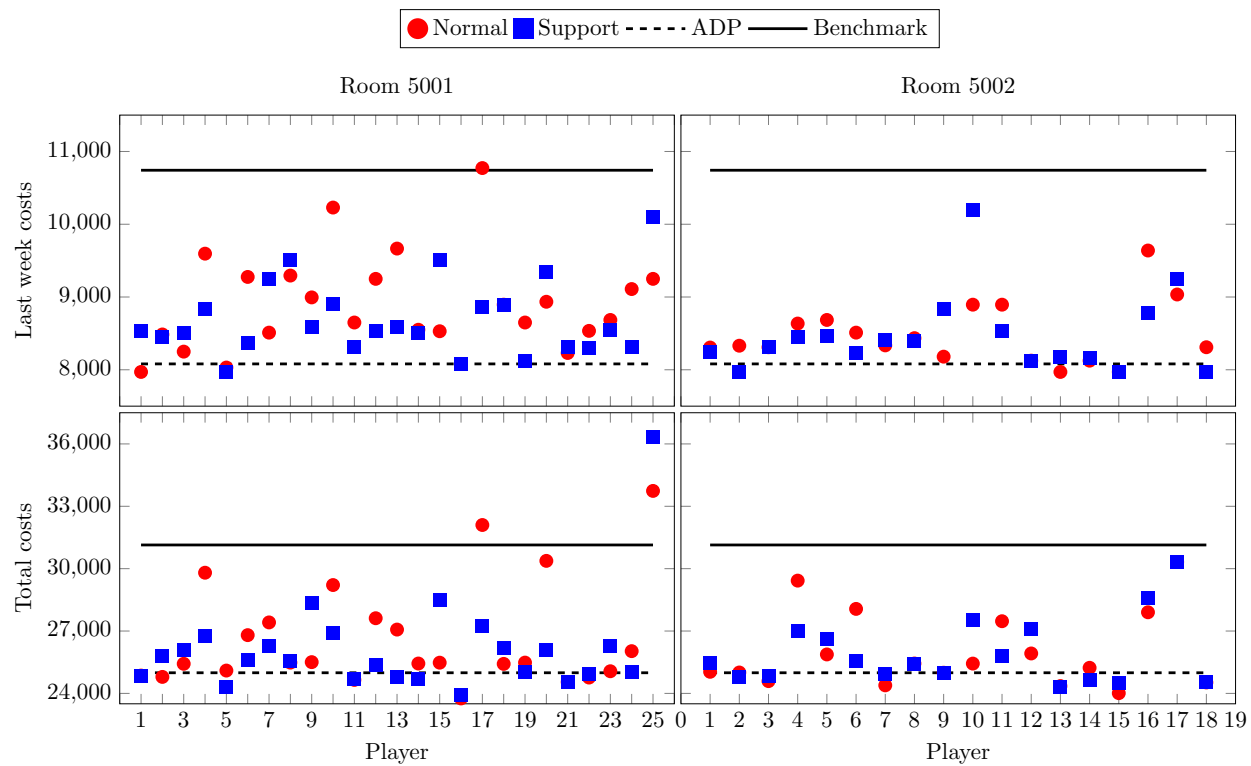

Figure 7.14: Performance of players in different rounds of Scenario A

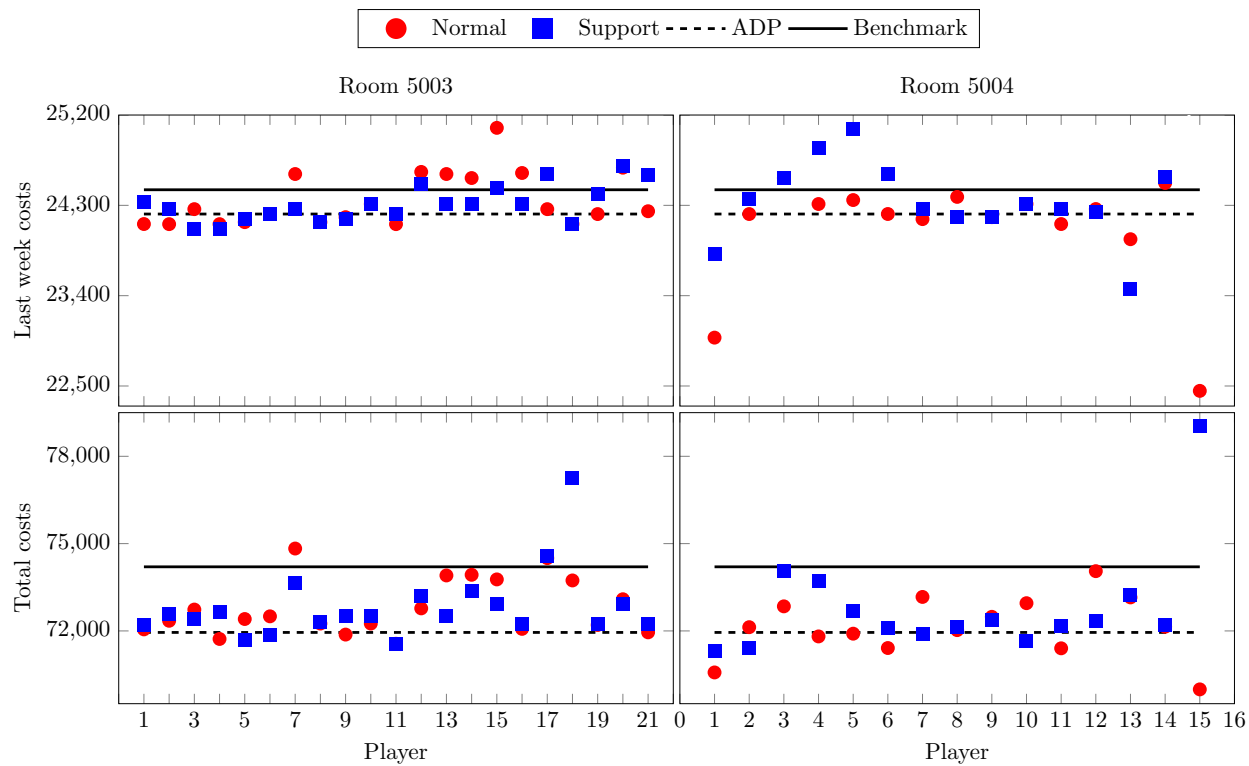

Figure 7.15: Performance of players in different rounds of Scenario B 
To study the difference between the performance of players in the normal and support rounds with respect to the ADP policy and the benchmark, we plot the individual costs of each player in Figures 7.14 and 7.15. In Scenario A (Figure 7.14), almost all players are within the costs of the benchmark heuristic and the ADP policy during the last week and in total. However, the difference between their normal and support round varies more in Room 5001 than in Room 5002. This observation seems to indicate that, once players played a support round, they play the normal round in a similar way. In Scenario B (Figure 7.15), more players are outside the range of the benchmark heuristic and the ADP policy. Furthermore, most players seem to improve on the second round they play (i.e., support round for Room 5003 and normal round for Room 5004). This may indicate that, with the more complex trade-offs of Scenario B, training pays off more than support.

Overall, in the games with first support and then normal rounds (i.e., Rooms 5002 and 5004 ), the sum of the costs in both rounds are significantly lower than in the rooms with the inverted sequence of rounds. In addition, the difference between players in these games are smaller. These two observations indicate that a round of support helps afterwards when no longer support is given. Moreover, we heard from students that they had to get adjusted to support information, and that they had to learn how to interpret it. It could be that, for Rooms 5001 and 5003, when students finally grasped how to use the support, there were no more rounds to play. Remind that the practice round does not offer support.

Even though we did not record qualitative information formally (e.g., briefing and de-briefing questionnaires or interviews), we heard several comments during the game session and talked with several students about their experience with it. In general, students were engaged with the game and were eager to make the best decisions possible. During the game, several students asked us (the game masters), for additional information about the game settings (e.g., release-day distributions, cleaning costs, etc.) to make better decisions. Furthermore, the competitive students were often looking at the leader-board and comparing themselves with others. Some minor bugs were discovered. The most severe bug was having a container hovering in the air after the it was still being dragged over the screen when the time limit was reached. The container could not be selected anymore in subsequent days. Players encountering this error had to re-start the game.

Besides a fun playing experience, students reported learning to make better decisions throughout the game. A large part of making better decisions, they claimed, was to know the costs for groups of containers. Even though all costs are given in the game (see Figure 7.4), properly using this information requires time. After some rounds, several students knew which combination of destinations was cheapest by barge and how many containers were needed to cover the setup costs of using the barge (i.e., barge transport being cheaper than truck transport). With respect to the support, some students reported it being hard to interpret and sometimes counter-intuitive. However, once they got used to it, some students said they could find the pattern (i.e., policy) that the support was hinting towards (e.g., postponing blue containers was more desirable than red ones).

To conclude, the test gaming session achieved its objectives and the game is able to raise awareness about anticipatory scheduling. Players were engaged with the game, the game play was intuitively clear, and many simultaneous players could participate in a single 
session. Competition was present throughout the gaming session and players were involved with achieving the best performance. The test scenarios were challenging and enabled us to study the behavior of participants, as observed in the variance of the results. Players reported that on-hand knowledge of the costs and identifying patterns of container arrivals were key to achieve a good performance. In addition, the results and observations of the test gaming session give rise to further improvements of the ADP policy (i.e., support) in the game. Good players were able to improve over time and even outperform ADP. This under-performance of ADP could be partly attributed to our VFA design. In the game, we apply the generic model of Chapter 2. However, a different VFA could be designed for the specific problem considered in the game. Consequently, better ADP performance and perhaps better support could be given to the players.

\subsection{Conclusions}

In this chapter, we designed a serious game to raise awareness about trade-offs in anticipatory scheduling of synchromodal transport and to educate on how to understand and optimize these trade-offs. We designed the game based on a common single-trip long-haul container transport problem where containers have different destinations and time-windows. The game can be played in different modes, which help the player to minimize the logistical costs to various extents, and has several mechanisms to foster their engagement and competitiveness. The game master can define various settings of the game, which allows him or her to have different purposes for different target audiences, such as LSP planners, operations management students, high-school students, or the general public. We described how the game master can achieve different purposes by defining the different settings. In test gaming sessions, we observed that the main differences among players occurred in: (i) how well they interpreted the idea of decision support (expected long-term impact of a decision), (ii) how often players consulted the costs information, and (iii) whether players identified the differences in arrival patterns of the different container types.

Even though our game design is a first step towards measuring the effectiveness of serious gaming in raising awareness and educating about trade-offs in freight transport, some limitations and extensions may need to be addressed first before further research can be done. For example, a limitation could be that practitioners are biased towards certain decisions given their normal working environment, which might differ from the game scenarios (e.g., different cost structure). Defining the settings of a game scenario should not only serve the purpose of the game master, but should also result in a game play that players deem as realistic enough. Furthermore, when the game is used for didactic purposes, the game master must carefully design game scenarios where the lesson can be attributed to the correct aspects of the game (e.g., performance of a player improved because of the use of the estimated future cost in the support mode and not because the orders arriving where easier than in the normal mode).

Currently, our game has only one intervention to support the player on having an anticipatory perspective in scheduling: the estimated future costs. An extension could be decision support in the form of automatic decisions that the player can choose. For example, a player can choose a certain type of container (e.g., green containers with a time-window 1) 
to be automatically assigned to the barge every day. This way, the player has time to focus on the difficult problems. Such extensions have been shown to improve the performance of players in similar serious games [25] and can be used for didactic purposes on the complexity of decisions. Working on these limitations and extensions can increase the research possibilities of our game. Furthermore, they can improve the game as a tool to facilitate the adoption of an anticipatory perspective when scheduling synchromodal transport operations. 


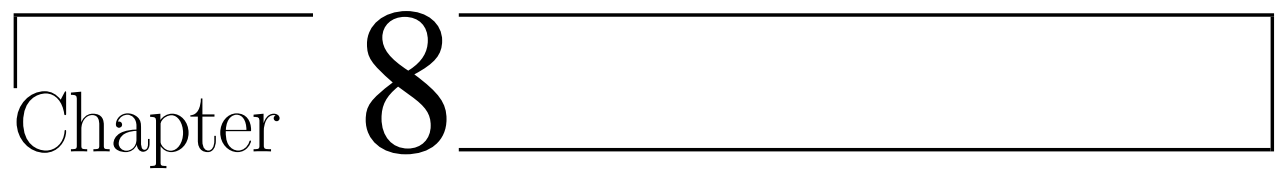

\section{Conclusions and Prospects}

In this chapter, we present the main conclusions of this dissertation. Furthermore, we provide an outlook for further research and for implementation of our methods. This chapter is organized in three parts. First, in Section 8.1, we recollect the main findings of our research chapters in the form of a response to the research questions posed in Chapter 1. Second, in Section 8.2, we discuss the implications of our findings for (i) the further development of scientific knowledge in areas related to our research, and (ii) the implementation of our research in practice. Third, in Section 8.3, we provide closing thoughts on the anticipatory scheduling of freight in synchromodal transport.

\subsection{Conclusions}

Our research was part of the project SynchromodalIT, which aimed to deliver, among others, scheduling methods to optimize the performance of synchromodal transport, and knowledge about the benefits of these methods compared to traditional scheduling methods for multimodal transport. When the existing scheduling methods for multi-modal transport were assessed for their usability in synchromodal transport, two main shortcomings were found. First, these methods typically focus on a single-period objective for an individual part of the transport network, such as scheduling drayage operations or scheduling freight transport in a highcapacity mode in a corridor. Second, the methods that consider a multi-period objective, and hence consider the evolution of the transport network through time, usually focus on producing a schedule up-front that is static, i.e., does not adapt to any form of uncertainty in the realization of the schedule. Based on these shortcomings, and considering that dynamic scheduling that looks ahead over time and over the network (or anticipatory scheduling as we defined in Chapter 1) is a key characteristic of synchromodality, we defined a three-fold research objective for the dissertation: (i) to develop mathematical models and heuristic algorithms that support anticipatory freight scheduling in synchromodal transport, (ii) to evaluate the output 
of our models and algorithms in terms of logistical costs, and (iii) to gain insights into the benefits of anticipatory decision making under different synchromodal transport networks.

To achieve our objective, we studied the scheduling of freight in synchromodal transport from four different perspectives. First, we studied the scheduling of freight in long-haul round-trip transport in Chapters 2 and 3. Second, we studied the scheduling of freight in long-haul multi-transfer transport in Chapter 4. Third, we studied the scheduling of freight in multi-terminal drayage transport in Chapter 5. Fourth, we studied the integrated scheduling of drayage and long-haul transport of freight in Chapter 6 . In the four perspectives, we developed scheduling methods comprised by a mathematical optimization model and a heuristic algorithm whose output is an anticipatory transport schedule for the available freights. To develop and evaluate such scheduling methods, as well as to gain insight into their use, we defined five research questions in Chapter 1. Even though these questions are answered throughout several chapters, in this chapter we summarize their response one by one.

RQ 1. What are the limitations of current scheduling methods for multi-modal transport with respect to synchromodality and what improvements are necessary?

The scheduling of freight in long-haul round-trip transport, studied in Chapters 2 and 3, and the scheduling of long-haul multi-transfer transport, studied in Chapter 4, belong both to the scheduling problem classes of DSND and stochastic freight transport. These problem classes have a vast body of knowledge, from which we learned that techniques such as MILP, in the case of DSND, and MDP, in the case of stochastic freight transport, are useful for modeling the evolution of the network through time and capturing the uncertainty from one decision moment to the next. However, there were two main shortcomings when we evaluated these methods for synchromodal transport. Within DSND, research that considers stochastic problems with re-planning decisions was limited compared to deterministic single-plan research. Within dynamic and stochastic freight transport, research that includes multiple modes, round-trips, and multi-period transport with transfers was limited, compared to research considering a single mode and one-period execution of decisions. Based on these observations, we identified to required improvements. The first improvement relates to the development of richer scheduling methods. Models should include stochastic freight demand, measure performance over a multi-period horizon for multiple modes, and capture the complex time-decision evolution of the network. Heuristics to solve these models for large instances should be able to overcome the challenges recognized in the literature, such as weak bounds, solutions' symmetry, and abundance of local-optima. The second improvement relates to the development of methodological insights on the design process of heuristic algorithms. Since the scheduling problems for each part correspond to the intersection of DSND problems with stochastic freight transport problems, the individual challenges mentioned before are intertwined and hence, new strategies to handle their combination are necessary.

The scheduling of drayage operations in synchromodal transport, studied individually in Chapter 5, and integrated with long-haul transport in Chapter 6, belongs to a special case of the VRP: the FTPDPTW. Although the VRP and FTPDPTW have been studied extensively, 
there has been limited research on the multiple attributes and decision characteristics of drayage operations. One of the least studied characteristic of drayage operations has been the choice of which terminal to bring a pre-haulage freight to for the start of its long-haul transport. This was most likely due to the traditional workings of multi-modal transport, where the long-haul mode is fixed up-front and hence the initial terminal for the long-haul fixed for a pre-haulage freight. However, the flexibility in mode choice, and hence choice of initial terminal, is a key characteristic of synchromodality. The first improvement necessary for the scheduling of drayage operations relates to the inclusion of attributes such as the flexibility in the terminal assignment for pre-haulage jobs. Including such flexibility (or such an additional decision) has an impact on the scheduling of all drayage operations, as well as on the long-haul transport. In the long-haul transport, the impact not only depends on which long-haul modes depart from terminals that pre-haulage freights were brought to, but also on the current circumstances of those terminals, such as available freights, disruptions, delays, etc. Since these circumstances are dependent on the long-haul scheduling decisions made before the drayage decisions, a link between the two decisions is necessary. Traditionally, DSND studies have included a link to the drayage in the form of costs and with a constraint in the number of trucks. However, DSND studies have not explicitly modeled the diversity of characteristics of drayage operations, which have been shown to account for a significant part of the total multi-modal costs. Therefore, the second improvement identified relates to the development of scheduling methods that integrate drayage and long-haul scheduling decisions.

RQ 2. How can anticipatory scheduling methods be designed for different parts of a synchromodal transport network?

The path of a freight in multi-modal transport can be divided into three parts: pre-haulage, which is the transport from the freight's origin to its first terminal (i.e., transfer); long-haul, which is the transport from the first terminal to the last one, including possible transfers in between; and the end-haulage, which is the transport from the last terminal to the freight's destination. For an LSP that schedules the transport of such freights (e.g., containers), each part differs in the type of decisions and their extent in the network and in time. In the prehaulage, the LSP has to decide on the truck and the time to pick-up a freight, as well as the terminal to bring this freight to. The terminal will influence the possible long-haul routes for this freight. In the long-haul, the LSP has to decide the services (i.e., modes, locations, and departure time) to use. In the end-haulage, the LSP has to decide on the truck and the time to transport the freight from its last terminal in the long-haul to its final destination. This last part has the "simplest" of the decisions of the three parts, since the previous decisions (i.e., final terminal of a freight) limit the options to consider. Furthermore, existing dynamic multimodal scheduling methods can be directly applied, since the only relation to synchromodality relates with the status (e.g., current location, delays, etc.) of the trucks carrying out the transport. For the initial two parts (i.e., the pre-haulage and the long-haul), however, the scheduling requires more than the use of existing methods for multi-modal transport.

Scheduling the long-haul of freights in synchromodal transport brings additional challenges 
to existing multi-modal methods. In Chapters 2 and 4, where we studied these type of decisions, we noticed three main challenges to tackle when modeling the problem. First, freights that arrive to the network are uncertain. The uncertainty is not only on the number of freights that arrive each period, but also on their characteristics. Second, the performance advantage of consolidating as many freights as possible in high-capacity modes can be conflicting with the optimization of performance over a multi-period horizon, since future freights may bring better consolidation opportunities throughout the network and throughout time. Third, the time-window of each freight restricts the immediate service that can be used for that freight, but also the posterior services and transfers that can be used depending on the immediate decision and the timing of services. To tackle the first two challenges of long-haul scheduling, we used MDP theory, which provides a framework to model dynamic and sequential decisions under uncertainty. Furthermore, the outcome of an MDP model is a policy, or decision function, that maps the state of the system (e.g., freights at each terminal, services available, etc.) to a decision that optimizes the performance over the planning horizon. Hence, the policy results in dynamic and optimal scheduling based on the outcome of the random variables. To tackle the third challenge, within the MDP framework, we used MILP, which provides a mathematical framework to formulate the evolution of the network considering quantitative constraints. MILP models have been successfully used in many DSND problems, and have the advantage of a large body of standardized algorithms to solve them.

Scheduling the pre-haulage in synchromodal transport brings two additional challenges to existing multi-modal methods. First, in Chapter 5, where we studied decisions regarding drayage operations, we noticed that the flexibility in synchromodal transport meant three simultaneous decisions in the drayage: (i) the timing of the pick-up and delivery of pre- and endhaulage freights, (ii) the routing of the trucks that transport them, and (iii) the selection of terminals for pre-haulage freights to start their long-haul. These decisions have a shorter time span than the long-haul decisions, and thus relatively less uncertainty. To model this scheduling problem, we used MILP. MILP has versatility for modeling many types of constraints and including categorization of freights and decisions, and is commonly used in related VRP and FTPDPTW problems. This versatility can be used to incorporate synchromodal characteristics into existing multi-modal scheduling methods. Second, in Chapter 6, where we studied integrated drayage and long-haul decisions, we noticed that the terminal selection decision depends not only on the drayage operations but also on the current and expected long-haul transport. The challenge lies in the relationship of the two transport operations, since the circumstances and expected performance of the long-haul decisions depend on the result of the drayage decisions. To tackle this challenge, we integrated the individual MILP model of Chapter 5 and the long-haul MDP model of Chapter 4 through an iterative simulation approach. The simulation approach has the advantage that the complex relations of the decisions for the two parts, and their recursive relation, can be analyzed without an explicit analytical formulation. However, the approach is heuristic in nature and therefore required the calibration of various components that join the scheduling of drayage transport with the long-haul transport. 
RQ 3. How can the anticipatory scheduling methods of $R Q 2$ be modified to handle large size instances efficiently?

For long-haul transport, we studied round-trips and multi-transfers in Chapters 2 and 4 , respectively. We modeled both parts as an MDP where decisions anticipate on future demand by including expected future performance in the optimization goal, but these models are solvable within reasonable time for tiny problem instances only. To overcome this, we designed ADP algorithms that approximate the expected future performance in each part by quantifying distinctive features of states into so-called basis functions, and learning a weight for each basis function per MDP stage (i.e., planning period). Even though the anticipatory mechanism of basis functions works well in many stochastic optimization problems, their design and the learning of their weights can be challenging. In Chapter 2, we exemplified the challenges when evaluating various sets of basis functions and showed how additional analysis besides statistical inference and simulation, such as categorization of states and relation of basis functions to such categories, were needed to select the best performing set of state-features. In addition to evaluating sets of basis functions, in Chapter 4, we discussed the challenge of learning the proper weights of basis functions. In multi-transfer transport, there are also multi-period traveling times, which we efficiently modeled using virtual timewindows in the state definition. Such a modeling approach, even though efficient in terms of variables, seemed to make the ADP algorithm flounder and end in local-optima when using traditional learning strategies such as exploitation and $\epsilon$-greedy exploration. To overcome this problem, we studied a method from Bayesian exploration, VPI, that escapes local-optima by incorporating the expected gain of knowledge to avoid such local-optima in subsequent decisions. However, the VPI method was designed for reinforcement learning problems structurally different from ours. The most important difference is that the original VPI method ignores the presence of a "physical" state, i.e., a state that determines what states can be observed in future stages (and the rewards of decisions in such states). Furthermore, it assumes an infinite horizon. We designed and evaluated various modifications to VPI, and showed that to successfully apply VPI in a finite-horizon ADP with physical states and with basis functions such as ours, exploring and updating the belief should be done slightly more conservative than in conventional VPI for reinforcement learning problems.

For the multi-terminal drayage transport problem studied in Chapter 5, we used MILP to model the problem where scheduling decisions anticipate on the performance of the subsequent long-haul transport by including a terminal assignment cost in the optimization goal. Although commercial MILP solvers increasingly expand the problem sizes they can handle, their computational efficiency in problems such as ours is still limited due to the highly symmetrical and, in some cases sparsely constrained, feasible decision space. Furthermore, in contrast to long-haul transport, pre-haulage transport requires decision making that is more frequent and hence requires faster solving times. To achieve fast and good solutions for our MILP model, we designed a matheuristic that iteratively confines the solution space of the MILP using problem-specific adaptations and fixing criteria, and based on the incumbent solutions, guides the subsequent iterations and solutions. The adaptations 
are additional constraints (similar to cutting planes) that reduce the size of the feasible decision space of the MILP based on knowledge of terminals available and time-windows of drayage requests. The fixing criteria are rules for defining the domain of variables based on the schedule before a re-scheduling event occurs. The adaptations and fixing criteria are based on our categorization of drayage requests. We studied the relation between the best adaptations and the dispersion of requests, and analyzed the performance of the fixing criteria in re-scheduling. For the integrated long-haul and drayage transport studied in Chapter 6 , we designed a simulation-based approach to integrate the matheuristic of the multi-terminal drayage transport and the ADP approach for long-haul multi-transfer transport.

RQ 4. How do anticipatory scheduling methods perform under different network settings and how do they compare to other benchmarks?

Our scheduling methods have two components: (i) mathematical models that yield optimal schedules but are computationally solvable for small problem instances only and (ii) heuristic algorithms to solve the models for large problem instances but that do not guarantee optimal solutions. To test their performance under different network settings and against other benchmarks, we carried out two types of numerical experiments. First, we tested accuracy and calibration of the algorithms to guarantee that the heuristics were as close to optimal as possible. Second, we tested savings of our heuristics compared to benchmarks. In the following, we present our main findings for each part of the network studied and for each type of experiment, where the word "instance" is used to refer to a particular setting of that part of the network.

For the long-haul round-trip transport problem from Chapters 2 and 3, we studied the accuracy of our ADP approach with respect to the optimal costs of the MDP model varying two network characteristics: destination balance and time-window length. We tested three sets of features and different settings for our ADP approach. The best set of features and settings had an average optimality gap between $1.3 \%$ and $6.5 \%$ among all instances tested. We observed that our ADP approach was most accurate in instances with longer time-windows. In our evaluation experiments in Chapter 3, we tested the savings of our ADP approach compared to a single-period optimization benchmark varying several network settings, such as destination balance, release-day and time-window length. For each network setting, various initial conditions were tested under categories that were different in the number of freights and the number of different destinations these freights had. Our ADP approach resulted in savings between $5.9 \%$ and $8.6 \%$ on average across initial conditions in all instances but one, where the savings where only of $1.2 \%$. This last instance was the one where freights had short time-windows and short release-days. Our approach achieved the largest savings when there was a large number of pre-announced orders (i.e., long release-day) and unbalanced destinations. With respect to initial conditions, we observed savings between $2.8 \%$ and $11.2 \%$ on average across all instances per category. Savings where larger in those categories with a medium number of freights and a low number of different destinations.

For the long-haul multi-transfer transport problem from Chapter 4, we studied the tuning of our ADP-VPI approach using three network topologies that differed in the number of 
transfers and services possible and the objective to maximize profit. We observed that to tune VPI in our ADP approach, there is an ideal ratio between two tunable parameters of the VPI method (i.e., the noise term and the initial covariance matrix), and that exploring and updating within VPI should be done slightly more conservative than in the original definition. In our evaluation experiments, we tested our tuned ADP-VPI approach against an anticipatory benchmark heuristic using the three networks topologies of the tuning experiments, introducing a restricted decision space (for computational purposes), and varying two freight demand characteristics: release-days and time-window lengths. The gains of our approach were between $1 \%$ and $272 \%$ with respect to the new benchmark in all network characteristics tested but one. In the most complex network (i.e., largest number of possible transfers and services) that had freight demand with short time-windows, our approach performed worse than the benchmark. The improvements were larger on networks that had few transfers, long time-windows and short release-days.

For the multi-terminal drayage transport problem from Chapter 5, we studied the use of our matheuristic approach in a static (i.e., one-plan only) and a dynamic (i.e., re-planning throughout the day) perspective in a time horizon of one day. In the experiments of the static perspective, we varied two characteristics of drayage requests: request location and time-windows. We studied several modifications to the MILP and matheuristic operators to tune our approach and compared them to a benchmark heuristic. Our approach resulted in savings between $3-4 \%$ for problem instances where requests are clustered to the central terminal or where they have tight time-windows. We observed that time-window preprocessing and pre-pairing of requests are effective mechanisms in our approach to achieve these savings. In instances with sparse requests and long time-windows, our approach was only slightly better than the benchmark heuristic. In our dynamic re-scheduling experiments, we observed that the savings of the matheuristic went up to $8 \%$. However, for instances with long-time windows and sparsely located customers, we had the same performance as the benchmark, for both static and dynamic scheduling.

For the integrated long-haul and drayage transport problem from Chapter 6 , we studied the calibration of our iterative simulation scheduling approach varying three instance characteristics: drayage location, drayage type, and long-haul destination. We observed that careful calibration per instance is required. Furthermore, we observed that our calibrated approach achieves lower total costs while having higher drayage costs. In other words, it seemed that drayage schedules that incur additional travel costs to bring freight to further terminals can result in significantly lower costs for the long-haul. Naturally, this observation depends on the cost settings of the network. In our evaluation experiments, we compared our approach against a benchmark using three cost setups for the drayage transport: low, normal, and high. We observed that our integration approach results in significant savings compared to a non-integrated approach: between $2 \%$ to $38 \%$ under the normal cost setup, over all instances, and between $6 \%$ and $49 \%$ in case of the low cost setup. In both the normal and low cost setup, savings where larger in instances with end-haulage dominant drayage types, even though in instances with pre-haulage dominant drayage types, the utilization of long-haul modes was better. In a low cost setup, our approached achieved savings in only $25 \%$ of the instances tested. 
RQ 5. How can serious gaming be used to facilitate the adoption of anticipatory synchromodal scheduling methods in practice?

Throughout this thesis, we observed that anticipatory scheduling methods sometimes produce counter-intuitive results due to their network-wide and look-ahead perspective on performance. Planners may have questions about these results or doubts about the workings of anticipatory scheduling methods that obtained them. To increase the understanding about our methods and to gain the trust required by planners, we designed a web-based serious game in Chapter 7 based on the single-trip problem and scheduling approach from Chapter 2. In this game, the player assumes the role of an LSP scheduler in charge of the transport of containers by barge or by truck, with the goal of minimizing logistical costs. The purpose of the game is to raise awareness about some of the trade-offs in anticipatory scheduling and to educate on how to understand and optimize these trade-offs.

The game has different playing modes in which the player can take different perspectives towards scheduling the transport of containers. Furthermore, it has several mechanisms that engage the competitiveness of players and that allow the game-master to define how the game develops. These elements of our game design allow us to raise awareness in various topics such as the future impact of decisions, the need to anticipate, and the benefits of using our scheduling methods (or the lack thereof). In addition, we discussed and exemplified how our game could be used for different purposes such as research and entertainment.

\subsection{Outlook for Research and Implementation}

The research findings in this thesis contributed to a better understanding of anticipatory scheduling of freight in synchromodal transport. However, these research findings also lead to new questions about the workings of our scheduling methods and about their use in practice. In this section, we present the prospects that we identified for future research and implementation. We organize the section in three parts. First, we discuss improvements to the heuristics in Section 8.2.1. Second, we discuss extensions to the models in Section 8.2.2. Third, we discuss practical issues that LSPs may find when translating our theoretical output into day-to-day practice in Section 8.2.3.

\subsubsection{Improvements to the Heuristics}

For each part of the network studied, we designed a heuristic to solve the optimization models for scheduling the transport of freight. These heuristics performed differently under different problem and algorithmic settings. In the following, we summarize the main observations about these differences and propose ways to improve them.

In the ADP approach tested in Chapters 2 and 3, we observed that relative differences in savings among initial conditions (i.e., categories of states) was bigger in large instances than in small ones. To improve the performance of the resulting policy of ADP in large instances and 
in different initial conditions, properties of these initial conditions and their relation to the basis functions must be studied. For example, a state with a low number of freights may require additional tuning of ADP parameters for the learning phase. Additionally, we observed that the difference between the resulting policy of ADP and the single-period optimization was smaller for the instance with freights that have short time-windows and release-days. In other words, with reduced flexibility in the transport of freight it seems that anticipating on future order arrivals pays off less. Investigating how problem characteristics influence the savings of ADP can lead to better designs. For example, we could use separate basis functions for different state categories of the same problem, thereby mixing a look-up table approach with the use of basis functions, or we could use pre-defined policies that do the scheduling under certain conditions, such as the low flexibility where acting myopic seemed to be best.

In the ADP approach tested in Chapter 4, we observed that in some networks, the impact of using VPI was larger than in others. This difference in performance among the networks may have to do with the "look-ahead possibilities" that different parts of the state space of a network have. For example, freights at terminals close to their origin have many aspects to look-ahead (e.g., possible transfers and combination of modes, future consolidation at following terminals, etc.) whereas freights at terminals close to their destination have less (e.g., when to deliver to final destination). Further research on how to differentiate look-ahead possibilities at different parts of the state space of a network, as well as how to estimate the downstream rewards based on these possibilities, may improve the resulting policy of our combined ADP-VPI design. One additional aspect that requires further attention is the set of restrictions we applied to make the large decision space smaller. Our main consideration to design the restrictions was to reduce computational time, but other (and certainly better) considerations need to be taken into account. Studying how to restrict the decision space in an ADP approach without loosing solution quality, for example using similar approaches as cutting planes for MILPs, could result in better and faster ADP policies. To summarize, the relation between the design of ADP-VPI approaches with the restricted decision spaces and network characteristics must be investigated.

In the matheuristic tested in Chapter 5 , differences in performance with respect to network characteristics were also observed. These differences may relate to the matheuristic operators and fixing criteria. These two matheuristic mechanisms are analogous to neighborhood operators in local search heuristics. This means that additional operators may be considered, as well as mechanisms that tune them or adapt them to network characteristics. For example, in random drayage locations, swapping the assigned terminal for pre-haulage freights with another one can result in lower terminal assignment cost or in better positioning of the truck for other drayage operations. In another example, in case of dynamic scheduling, fixing a route with many requests may perform better in instances with tight time-windows, and thus should be used more often than other fixing criteria throughout the day. This preference can be determined with some weighted selection procedure that adapts to the instance characteristics. The benefits of such additions should be further studied.

In the iterative simulation-based heuristic for the integrated scheduling from Chapter 6 , we observed that the savings of our approach differ with respect to the cost settings in the network. Although savings were achieved in every instance that had higher long-hauls costs com- 
pared to drayage costs, in instances with higher drayage costs, our approach did not achieve savings most of the time. A possible reason may be that better calibration is required, since we used the calibrated settings for higher long-haul costs. Another possible reason is that the sequential integration is not sufficient for achieving a good performance in these instances. For example, the resulting policy of ADP may have been learned under circumstances that capture only part of the overall problem. If, after observing the behavior of this resulting policy (e.g., through a simulation), new parts of the problem can be re-learned in ADP, the resulting policy may improve. Thus, a guideline for further research is to go beyond the sequential integration tested and into the iterative integration approach proposed. To go into this direction, several guidelines were proposed. For example, defining the long-haul probabilities based on simulation observations may result in a better trade-off between drayage operation costs and long-haul terminal assignment costs. Additionally, designing VFA learning mechanisms for ADP that are able to cope with situations that will be faced in the drayage operations may improve the resulting policy. To summarize, an iterative combination of ADP learning, resulting policy testing in the overall problem, and ADP re-learning must be investigated.

\subsubsection{Extensions of the Models}

In each part of the synchromodal network, we defined boundaries and assumptions about the scheduling problems encountered. Although some of the boundaries and assumptions were not restrictive and can easily be relaxed, as we explained in each chapter and their appendices, some others might not. In the following, we briefly discuss these limitations and propose extensions that are interesting to study from both a theoretical and practical perspective.

In the MDP model and ADP approach for long-haul round-trip and multi-transfer transport in Chapters 2 and 4, respectively, we considered uncertainty only in the freight demand, i.e., variable number of freights arriving and variable characteristics of freight. Naturally, there are many other sources of uncertainty that can be incorporated, and that can be anticipated upon, to improve performance over the network and over time. For example, uncertainty in the capacity of transport services or in their traveling times can have a significant influence on postponement decisions, especially when considering tight time-windows of freight. In both models, we considered transport restrictions mostly from the demand side (e.g., release-day, time-window length, destination), but less from the supply side (e.g., capacity). Some restrictions such as minimum use of a transport service, loading sequence for a barge, maximum holding capacity of a terminal, are challenging to include in models since they increase the complexity of the decision space (which we recommend to study better and try to simplify in the improvements to the heuristics) but can determine whether improvements in performance can be achieved.

In the dynamic MILP model and matheuristic about multi-terminal drayage operations in Chapter 5, we considered a generic definition of requests and resources, which handles the richness of characteristics in this special case of the static VRP. However, for a dynamic instance, an additional characteristic is crucial: the re-scheduling trigger. In our study, we considered only one trigger for re-scheduling: a fixed time interval. Other triggers, such as change in the status of trucks, traffic data, or cancellation of customer requests, can be interesting to study in relation to network characteristics. Different triggers for re-scheduling 
may require different modeling considerations. For example, if the trigger for re-planning is a delay due to increased traffic, suddenly the resource becomes uncertain and can or cannot be used for fulfilling the existing schedule. New questions arise such as whether the previous schedule should remain, what to do with the resource, etc. These questions correspond to new objectives and new decisions. For example, a new, additional, objective may be to minimize the risk of a schedule not being completed within a time-window, with a decision on the amount of slack time to include in a route.

In the iterative simulation approach for the integrated scheduling of long-haul transport and drayage transport in Chapter 6, our approach of integration is explicit on one side and implicit on the other. Drayage transport has an explicit objective component considering the performance of the long-haul, whereas long-haul transport works on an implicit input from the performance of drayage transport. This way of integration may be biased towards the long-haul performance, and makes our approach under-perform in networks where the drayage costs dominate, as we saw in our experiments. Furthermore, the implicit input from the drayage operations in the long-haul transport is in the form of empirical probability distributions that are used to determine a long-haul policy per terminal, and to estimate terminal assignment costs. This policy is determined for all states possible in the empirical distributions for each single terminal. However, for scheduling both drayage operations and long-haul transport, all terminal policies must be used simultaneously. It could happen then, that the combination of these policies leads to a situation where a terminal faces a state it has not seen before, thus not being able to provide the drayage operations with a correct estimated cost. Investigating methods for an equal and explicit integration of the two sequential transport processes is challenging due to the uncertainty in the arrival of freights and the decisions of the first of the two processes, but is also promising for LSPs in situations such as in city logistics where the intra-city distribution may be a more expensive component than inter-city distribution.

\subsubsection{Implementation Aspects}

The mathematical models and heuristic algorithms that comprise our scheduling methods may require tuning and, in some cases, extensions before implementing them in practice. Independent of these implementation issues, to which we already gave advice in the chapters and in the previous sections, LSPs face two more challenges in implementation: defining the input data and interpreting the output schedule. In the following, we elaborate on these two challenges from a practical perspective and also from a scientific perspective that requires further research.

The input data for scheduling transport processes is a complex mix of information that is known with certainty (e.g., terminal location), information that is partly known (e.g., characteristics of freights already booked), and information that is known probabilistically (e.g., number of freights next week). In our models, the input parameters we consider to be known with certainty, such as logistical costs, capacities, and service between terminals, are most of the time straightforward to define. Probabilistic information, such as distributions of freight arrivals and their characteristics, are more difficult to define. We model stochasticity using empirical distributions, meaning that we do not make any assumption on how the random variables behave. Such empirical distributions can be derived from historical data 
or from predictive analytics. The challenge is on when to update these distributions, for example, in case of seasonality in demand. This challenge is related to the definition of the planning horizon, since after the horizon has passed, new input parameters must be entered. The question of when to update the input parameters, or how robust the output of our scheduling methods is to changes of these parameters, are research opportunities that can generate insights into the practical use of our anticipatory scheduling methods.

For all scheduling methods, the main output are the decisions assigning freights to transport modes, transfers, routes, etc. However, there are additional outputs, such as the estimated future costs we use for the serious game, that may help the LSP make decisions when the decisions are not fully automated or when there is an event that was not explicitly modeled (e.g., disruption of a terminal for a fraction of time). This last part about unforeseen circumstances relates to the robustness of our methods to changes in stochastic parameters within the planning horizon. The output policy of our scheduling methods for the long-haul, for example, can still be used as a heuristic solution to such circumstances. The question remains on whether using it is a good idea, or another re-planning method is necessary. Such an investigation can yield insight into the limits of our methods and required new approaches. In addition, for our scheduling methods for long-haul transport, the output was a policy for a given finite horizon (e.g., possible decisions for the next week). This output was learned from a given initial state for a given finite horizon, and was evaluated based on the same horizon length and initial state. Obviously, this might be different in a practical implementation, where more frequent updating of the policy or learning with randomized states might be preferable.

\subsection{Closing Thoughts}

In this thesis, we exemplified how synchromodal transport offers the potential to reduce logistical costs, especially through better scheduling utilizing the increased decision flexibility. We developed anticipatory scheduling methods to improve the overview and control of network-wide circumstances and performance in synchromodal transport. Our work provides a step towards fulfilling the operational requirements that, together with the fulfillment of infrastructure and policy requirements, can make synchromodal transport succeed in practice. Furthermore, the discussion of our optimization models, heuristics, and numerical results provides new scientific knowledge and guidelines for further investigations in the scheduling of transport problems. To conclude, this dissertation has laid important foundations in the anticipatory scheduling of freight in multi-modal transport. 


\section{Bibliography}

[1] J. Andersen, T. G. Crainic, and M. Christiansen. "Service network design with asset management: Formulations and comparative analyses". In: Transportation Research Part C: Emerging Technologies 17.2 (2009). Selected papers from the Sixth Triennial Symposium on Transportation Analysis (TRISTAN VI), pp. $197-207$. DOI: http://dx.doi.org/10.1016/j.trc.2008.10.005.

[2] J. Andersen, T. G. Crainic, and M. Christiansen. "Service network design with management and coordination of multiple fleets". In: European Journal of Operational Research 193.2 (2009), pp. 377 -389. DOI: http://dx.doi.org/10.1016/j.ejor. 2007.10.057.

[3] J. Andersen, M. Christiansen, T. G. Crainic, and R. Gronhaug. "Branch and Price for Service Network Design with Asset Management Constraints". In: Transportation Science 45.1 (2011), pp. 33-49. DOI: 10.1287/trsc.1100.0333.

[4] R. Bai, S. W. Wallace, J. Li, and A. Y.-L. Chong. "Stochastic service network design with rerouting". In: Transportation Research Part B: Methodological 60 (2014), pp. 50 -65. DOI: http://dx.doi.org/10.1016/j.trb.2013.11.001.

[5] B. Behdani, Y. Fan, B. Wiegmans, and R. Zuidwijk. "Multimodal schedule design for synchromodal freight transport systems." In: European Journal of Transport 8 Infrastructure Research 16.3 (2016).

[6] R. W. Bent and P. V. Hentenryck. "Scenario-Based Planning for Partially Dynamic Vehicle Routing with Stochastic Customers". In: Operations Research 52.6 (2004), pp. 977-987. DOI: 10.1287/opre.1040.0124.

[7] G. Berbeglia, J.-F. Cordeau, and G. Laporte. "Dynamic pickup and delivery problems". In: European Journal of Operational Research 202.1 (2010), pp. 8 -15. DOI: http://dx.doi.org/10.1016/j.ejor.2009.04.024.

[8] C. Bierwirth, T. Kirschstein, and F. Meisel. "On Transport Service Selection in Intermodal Rail/Road Distribution Networks". In: Business Research 5.2 (2012), pp. 198-219. DOI: 10.1007/BF03342738.

[9] B. Bouzaiene-Ayari, C. Cheng, S. Das, R. Fiorillo, and W. B. Powell. "From Single Commodity to Multiattribute Models for Locomotive Optimization: A Comparison 
of Optimal Integer Programming and Approximate Dynamic Programming". In: Transportation Science 50.2 (2016), pp. 366-389. DOI: 10.1287/trsc.2014.0536.

[10] K. Braekers, A. Caris, and G. Janssens. "Integrated planning of loaded and empty container movements". English. In: OR Spectrum 35.2 (2013), pp. 457-478. DOI: $10.1007 / \mathrm{s} 00291-012-0284-5$.

[11] E. Buiel, G. Visschedijk, L. Lebesque, I. Lucassen, B. van Riessen, A. van Rijn, and G. te Brake. "Synchro mania-design and evaluation of a serious game creating a mind shift in transport planning". In: 46th International Simulation and Gaming Association Conference, ISAGA. 2015, pp. 1-12.

[12] A. Caris and G. Janssens. "A local search heuristic for the pre- and end-haulage of intermodal container terminals". In: Computers \& Operations Research 36.10 (2009), pp. 2763 -2772. DOI: http://dx.doi.org/10.1016/j.cor.2008.12.007.

[13] A. Caris, C. Macharis, and G. K. Janssens. "Decision support in intermodal transport: A new research agenda". In: Computers in Industry 64.2 (2013). Decision Support for Intermodal Transport, pp. 105 -112. DOI: http://dx.doi.org/10.1016/j. compind.2012.12.001.

[14] J.-F. Cordeau, G. Laporte, M. W. Savelsbergh, and D. Vigo. "Vehicle Routing". In: Transportation. Ed. by C. Barnhart and G. Laporte. Vol. 14. Handbooks in Operations Research and Management Science. Elsevier, 2007. Chap. 6, pp. 367 -428. DOI: http://dx.doi.org/10.1016/S0927-0507 (06)14006-2.

[15] F. Corman and L. Meng. "A review of online dynamic models and algorithms for railway traffic management". In: Intelligent Transportation Systems, IEEE Transactions on 16.3 (2015), pp. 1274-1284.

[16] T. G. Crainic, M. Gendreau, and J. M. Farvolden. "A Simplex-Based Tabu Search Method for Capacitated Network Design". In: INFORMS Journal on Computing 12.3 (2000), pp. 223-236. DOI: 10.1287/ijoc.12.3.223.12638.

[17] T. G. Crainic, M. Gendreau, and J.-Y. Potvin. "Intelligent freight-transportation systems: Assessment and the contribution of operations research". In: Transportation Research Part C: Emerging Technologies 17.6 (2009), pp. 541 -557. DOI: https: //doi.org/10.1016/j.trc.2008.07.002.

[18] T. G. Crainic, M. Hewitt, and W. Rei. "Scenario grouping in a progressive hedgingbased meta-heuristic for stochastic network design". In: Computers 86 Operations Research 43 (2014), pp. 90 -99. DOI: http://dx.doi.org/10.1016/j.cor. 2013. 08.020 .

[19] T. G. Crainic and K. H. Kim. "Chapter 8 Intermodal Transportation". In: Transportation. Ed. by C. Barnhart and G. Laporte. Vol. 14. Handbooks in Operations Research and Management Science. Elsevier, 2007, pp. 467 -537. DOI: http://dx.doi.org/10.1016/S0927-0507(06)14008-6.

[20] T. G. Crainic and B. Montreuil. "Physical Internet Enabled Hyperconnected City Logistics". In: Transportation Research Procedia 12 (2016). Tenth International 
Conference on City Logistics 17-19 June 2015, Tenerife, Spain, pp. 383 -398. DOI: https://doi.org/10.1016/j.trpro.2016.02.074.

[21] T. G. Crainic, P. Dell'Olmo, N. Ricciardi, and A. Sgalambro. "Modeling dryport-based freight distribution planning". In: Transportation Research Part C: Emerging Technologies 55 (2015). Engineering and Applied Sciences Optimization (OPT-i) - Professor Matthew G. Karlaftis Memorial Issue, pp. 518 -534. DOI: http://dx.doi.org/10.1016/j.trc.2015.03.026.

[22] L. C. Dall'Orto, T. G. Crainic, J. E. Leal, and W. B. Powell. "The single-node dynamic service scheduling and dispatching problem". In: European Journal of Operational Research 170.1 (2006), pp. 1 -23. DOI: http://dx.doi.org/10.1016/ j.ejor.2004.06.016.

[23] R. Dearden, N. Friedman, and D. Andre. "Model Based Bayesian Exploration". In: Proceedings of the Fifteenth Conference on Uncertainty in Artificial Intelligence. UAI'99. Stockholm, Sweden: Morgan Kaufmann Publishers Inc., 1999, pp. 150-159.

[24] DHL Customer Solutions \& Innovation. DHL Logistics Trend Radar. 2016. URL: http : / / www . dhl . com / en / about_us / logistics_insights / dhl _ trend_ research/trendradar.html.

[25] A. Dobrkovic, L. Liu, M.-E. Iacob, and J. van Hillegersberg. "Intelligence Amplification Framework for Enhancing Scheduling Processes". In: Advances in Artificial Intelligence - IBERAMIA 2016. Ed. by M. Montes y Gómez, H. J. Escalante, A. Segura, and J. d. D. Murillo. Cham: Springer International Publishing, 2016, pp. 89-100. DOI: 10.1007/978-3-319-47955-2_8.

[26] A. L. Erera and K. R. Smilowitz. "Intermodal Drayage Routing and Scheduling". In: Intelligent Freight Transportation. Ed. by P. A. Ioannou. Vol. 27. CRC Press, 2008. Chap. 11, pp. 171-188.

[27] A. Escudero, J. Muñuzuri, J. Guadix, and C. Arango. "Dynamic approach to solve the daily drayage problem with transit time uncertainty". In: Computers in Industry 64.2 (2013). Decision Support for Intermodal Transport, pp. 165 -175. DOI: http://dx.doi.org/10.1016/j. compind.2012.11.006.

[28] European Commission. White Paper on transport Roadmap to a single European transport area Towards a competitive and resource-efficient transport system. 2011. DOI: $10.2832 / 30955$.

[29] European Commission. EU transport in figures - Statistical Poketbook 2016. Publications Office of the European Union, 2016. DOI: 10.2832/809634.

[30] European Commission. The implementation of the 2011 White Paper on Transport "Roadmap to a Single European Transport Area towards a competitive and resourceefficient transport system" five years after its publication: achievements and challenges. 2016.

[31] European Environment Agency. Energy efficiency and specific CO2 emissions. 2017. URL: http : / / www . eea . europa . eu / data-and-maps / indicators / 
energy - efficiency - and-specific-co2-emissions / energy-efficiencyand-specific-co2-9.

[32] European Technology Platform ALICE. Research \& Innovation Roadmap - Corridors, Hubs and Synchromodality. 2015. URL: http://www.etp-logistics.eu/?page_ id $=292$.

[33] Eurostat. Freight transport statistics - modal split. 2016. URL: http://ec.europa. eu / eurostat / statistics - explained / index . php / Freight _ transport _ statistics_-_modal_split.

[34] A. Faria, D. Hutchinson, W. J. Wellington, and S. Gold. "Developments in Business Gaming: A Review of the Past 40 Years". In: Simulation 8 Gaming 40.4 (2009), pp. 464-487. DOI: 10.1177/1046878108327585.

[35] P. Francis, G. Zhang, and K. Smilowitz. "Improved modeling and solution methods for the multi-resource routing problem". In: European Journal of Operational Research 180.3 (2007), pp. 1045 -1059. DOI: http://dx.doi.org/10.1016/j. ejor.2006.03.054.

[36] M. Ghane-Ezabadi and H. A. Vergara. "Decomposition approach for integrated intermodal logistics network design". In: Transportation Research Part E: Logistics and Transportation Review 89 (2016), pp. 53 -69. DOI: http://dx.doi.org/10. 1016/j.tre.2016.02.009.

[37] G. Ghiani, E. Manni, and B. W. Thomas. "A Comparison of Anticipatory Algorithms for the Dynamic and Stochastic Traveling Salesman Problem". In: Transportation Science 46.3 (2012), pp. 374-387. DOI: 10.1287/trsc.1110.0374.

[38] G. Ghiani, E. Manni, A. Quaranta, and C. Triki. "Anticipatory algorithms for same-day courier dispatching". In: Transportation Research Part E: Logistics and Transportation Review 45.1 (2009), pp. 96 -106. DOI: http://dx.doi.org/10. 1016/j.tre.2008.08.003.

[39] G. A. Godfrey and W. B. Powell. "An Adaptive Dynamic Programming Algorithm for Dynamic Fleet Management, II: Multiperiod Travel Times". In: Transportation Science 36.1 (2002), pp. 40-54. DOI: 10.1287/trsc.36.1.40.572.

[40] J. C. Goodson, B. W. Thomas, and J. W. Ohlmann. "Restocking-Based Rollout Policies for the Vehicle Routing Problem with Stochastic Demand and Duration Limits". In: Transportation Science 50.2 (2016), pp. 591-607. DOI: 10.1287/trsc. 2015.0591.

[41] W. J. A. van Heeswijk, M. R. K. Mes, and J. M. J. Schutten. "The Delivery Dispatching Problem with Time Windows for Urban Consolidation Centers". In: Transportation Science Articles in advance (2017). DOI: 10.1287/trsc.2017.0773.

[42] L. Heilig, E. Lalla-Ruiz, and S. Voß. "port-IO: an integrative mobile cloud platform for real-time inter-terminal truck routing optimization". In: Flexible Services and Manufacturing Journal (2017). DOI: 10.1007/s10696-017-9280-z. 
[43] A. Hoff, A.-G. Lium, A. Lokketangen, and T. G. Crainic. "A metaheuristic for stochastic service network design". English. In: Journal of Heuristics 16.5 (2010), pp. 653-679. DOI: $10.1007 / \mathrm{s} 10732-009-9112-8$.

[44] T. Hülsmann. Logistics 4.0 and The Internet of Things. Workshop "Platforms for connected Factories of the Future". 2015.

[45] A. Imai, E. Nishimura, and J. Current. "A Lagrangian relaxation-based heuristic for the vehicle routing with full container load". In: European Journal of Operational Research 176.1 (2007), pp. 87 -105. DOI: http://dx.doi.org/10.1016/j.ejor. 2005.06 .044 .

[46] Inchainge B.V. The Fresh Connection. 2018. uRL: https://goo.gl/Bm1KUH.

[47] M. Janic. "Modelling the full costs of an intermodal and road freight transport network". In: Transportation Research Part D: Transport and Environment 12.1 (2007), pp. 33 -44. DOI: http://dx.doi.org/10.1016/j.trd.2006.10.004.

[48] J. Konings. Intermodal barge transport: network design, nodes and competitiveness. Ph.D. thesis. TU Delft, Delft University of Technology, 2009.

[49] S. Kurapati, I. Kourounioti, H. Lukosch, L. Tavasszy, and A. Verbraeck. "Fostering Sustainable Transportation Operations through Corridor Management: A Simulation Gaming Approach". In: Sustainability 10.2 (2018), p. 455. DOI: 10.3390/ su10020455.

[50] L. Lebesque, J. van Meijeren, M. de Boer, and S. Kosterman. Serious gaming for logistics. Preparing for the future. Tech. rep. TNO, 2017.

[51] L. Li, R. R. Negenborn, and B. D. Schutter. "Intermodal freight transport planning A receding horizon control approach". In: Transportation Research Part C: Emerging Technologies 60 (2015), pp. 77 -95. DOI: http://dx.doi.org/10.1016/j.trc. 2015.08 .002 .

[52] A.-G. Lium, T. G. Crainic, and S. W. Wallace. "A Study of Demand Stochasticity in Service Network Design". In: Transportation Science 43.2 (2009), pp. 144-157. DOI: $10.1287 / \operatorname{trsc} .1090 .0265$.

[53] H. K. Lo, K. An, and W. H. Lin. "Ferry service network design under demand uncertainty". In: Transportation Research Part E: Logistics and Transportation Review 59 (2013), pp. 48 -70. DOI: http://dx.doi.org/10.1016/j.tre. 2013. 08.004.

[54] C. Macharis and Y. Bontekoning. "Opportunities for OR in intermodal freight transport research: A review". In: European Journal of Operational Research 153.2 (2004). Management of the Future MCDA: Dynamic and Ethical Contributions, pp. 400 -416. DOI: http://dx.doi.org/10.1016/S0377-2217(03)00161-9.

[55] W. G. Macready and D. H. Wolpert. "Bandit problems and the exploration/exploitation tradeoff". In: IEEE Transactions on evolutionary computation 2.1 (1998), pp. 2-22. 
[56] A. del Mar Agamez-Arias and J. Moyano-Fuentes. "Intermodal transport in freight distribution: a literature review". In: Transport Reviews 0.0 (2017), pp. 1-26. DOI: 10.1080/01441647.2017.1297868.

[57] N. Marković, Željko Drobnjak, and P. Schonfeld. "Dispatching trucks for drayage operations". In: Transportation Research Part E: Logistics and Transportation Review 70 (2014), pp. 99 -111. DOI: http://dx.doi.org/10.1016/j.tre.2014.06.016.

[58] S. A. Meijer, I. S. Mayer, J. van Luipen, and N. Weitenberg. "Gaming Rail Cargo Management: Exploring and Validating Alternative Modes of Organization". In: Simulation \& Gaming 43.1 (2012), pp. 85-101. DOI: 10.1177/1046878110382161.

[59] M. R. K.. Mes and M.-E. Iacob. "Synchromodal Transport Planning at a Logistics Service Provider". In: Logistics and Supply Chain Innovation: Bridging the Gap between Theory and Practice. Ed. by H. Zijm, M. Klumpp, U. Clausen, and t. M. Hompel. Cham: Springer International Publishing, 2016. Chap. Synchromodal Transport Planning at a Logistics Service Provider, pp. 23-36. DOI: 10.1007/9783-319-22288-2_2.

[60] M. R. K. Mes, M.-E. Iacob, and J. van Hillegersberg. "A Distributed Barge Planning Game". In: Frontiers in Gaming Simulation: 44th International Simulation and Gaming Association Conference, ISAGA 2013 and 17th IFIP WG 5.7 Workshop on Experimental Interactive Learning in Industrial Management, Stockholm, Sweden, June 24-28, 2013. Revised Selected Papers. Ed. by S. A. Meijer and R. Smeds. Cham: Springer International Publishing, 2014, pp. 214-221. DOI: 10.1007/978-3319-04954-0_25.

[61] M. R. K. Mes and A. E. Pérez Rivera. "Approximate Dynamic Programming by Practical Examples". In: Markov Decision Processes in Practice. Ed. by R. J. Boucherie and N. M. van Dijk. Cham: Springer International Publishing, 2017, pp. 63-101. DOI: 10.1007/978-3-319-47766-4_3.

[62] D. R. Michael and S. L. Chen. Serious Games: Games That Educate, Train, and Inform. Muska \& Lipman/Premier-Trade, 2005.

[63] L. Moccia, J.-F. Cordeau, G. Laporte, S. Ropke, and M. P. Valentini. "Modeling and solving a multimodal transportation problem with flexible-time and scheduled services". In: Networks 57.1 (2011), pp. 53-68. DOI: 10.1002/net.20383.

[64] B. Montreuil, R. D. Meller, and E. Ballot. "Physical Internet Foundations". In: Service Orientation in Holonic and Multi Agent Manufacturing and Robotics. Ed. by T. Borangiu, A. Thomas, and D. Trentesaux. Berlin, Heidelberg: Springer Berlin Heidelberg, 2013, pp. 151-166. DOI: 10.1007/978-3-642-35852-4_10.

[65] J. Nabais, R. Negenborn, R. C. Benítez, and M. A. Botto. "Achieving transport modal split targets at intermodal freight hubs using a model predictive approach". In: Transportation Research Part C: Emerging Technologies 60 (2015), pp. 278 -297. DOI: http://dx.doi.org/10.1016/j.trc.2015.09.001. 
[66] J. Nossack and E. Pesch. "A truck scheduling problem arising in intermodal container transportation". In: European Journal of Operational Research 230.3 (2013), pp. 666 680. DOI: http://dx.doi.org/10.1016/j. ejor.2013.04.042.

[67] C. Novoa and R. Storer. "An approximate dynamic programming approach for the vehicle routing problem with stochastic demands". In: European Journal of Operational Research 196.2 (2009), pp. 509 -515. DOI: http://dx.doi.org/10. $1016 / j$.ejor.2008.03.023.

[68] F. Pasin and H. Giroux. "The impact of a simulation game on operations management education". In: Computers $\&$ Education 57.1 (2011), pp. 1240 -1254. DOI: https: //doi.org/10.1016/j.compedu.2010.12.006.

[69] A. E. Pérez Rivera and M. R. K. Mes. "Dynamic Multi-period Freight Consolidation". English. In: Computational Logistics: 6th International Conference, ICCL 2015, Delft, The Netherlands, September 23-25, 2015, Proceedings. Ed. by F. Corman, S. Voß, and R. R. Negenborn. Vol. 9335. Lecture Notes in Computer Science. Springer International Publishing, 2015, pp. 370-385. DOI: 10.1007/978-3-31924264-4_26.

[70] A. E. Pérez Rivera and M. R. K. Mes. "Service and Transfer Selection for Freights in a Synchromodal Network". In: Computational Logistics: 7th International Conference, ICCL 2016, Lisbon, Portugal, September 7-9, 2016, Proceedings. Ed. by A. Paias, M. Ruthmair, and S. Voß. Cham: Springer International Publishing, 2016, pp. 227-242. DOI: $10.1007 / 978-3-319-44896-1 \_15$.

[71] A. E. Pérez Rivera and M. R. K. Mes. "Anticipatory freight selection in intermodal long-haul round-trips". In: Transportation Research Part E: Logistics and Transportation Review 105.Supplement C (2017), pp. 176 -194. DOI: https: //doi.org/10.1016/j.tre.2016.09.002.

[72] A. E. Pérez Rivera and M. R. K. Mes. "Anticipatory Scheduling of Freight in a Synchromodal Transportation Network". Beta Working Paper 533. 2017.

[73] A. E. Pérez Rivera and M. R. K. Mes. "Scheduling Drayage Operations in Synchromodal Transport". In: Computational Logistics: 8th International Conference, ICCL 2017, Southampton, UK, October 18-20, 2017, Proceedings. Ed. by T. Bektaş, S. Coniglio, A. Martinez-Sykora, and S. Voß. Cham: Springer International Publishing, 2017, pp. 404-419. DOI: 10.1007/978-3-319-68496-3_27.

[74] A. E. Pérez Rivera and M. R. K. Mes. "Integrated scheduling of drayage and long-haul operations in synchromodal transport". Working Paper. 2018.

[75] V. Pillac, M. Gendreau, C. Guéret, and A. L. Medaglia. "A review of dynamic vehicle routing problems". In: European Journal of Operational Research 225.1 (2013), pp. 1 -11. DOI: http://dx.doi.org/10.1016/j.ejor.2012.08.015.

[76] W. B. Powell. Approximate Dynamic Programming: Solving the Curses of Dimensionality. Vol. 1. John Wiley \& Sons, 2007. 
[77] W. B. Powell. Approximate Dynamic Programming: Solving the Curses of Dimensionality. Second Edition. John Wiley \& Sons Inc., 2011.

[78] W. B. Powell, B. Bouzaiene-Ayari, and H. P. Simao. "Chapter 5 Dynamic Models for Freight Transportation". In: Transportation. Ed. by C. Barnhart and G. Laporte. Vol. 14. Handbooks in Operations Research and Management Science. Elsevier, 2007, pp. 285 -365. DOI: http://dx.doi.org/10.1016/S0927-0507(06)14005-0.

[79] W. B. Powell and I. O. Ryzhov. Optimal Learning. John Wiley \& Sons, Inc., 2012, pp. i-xix. DOI: 10.1002/9781118309858.

[80] W. B. Powell, H. P. Simao, and B. Bouzaiene-Ayari. "Approximate dynamic programming in transportation and logistics: a unified framework". In: EURO Journal on Transportation and Logistics 1.3 (2012), pp. 237-284. DOI: 10.1007/s13676012-0015-8.

[81] J. Raghothama and S. A. Meijer. "A Review of Gaming Simulation in Transportation". In: Frontiers in Gaming Simulation: 44th International Simulation and Gaming Association Conference, ISAGA 2013 and 17th IFIP WG 5.7 Workshop on Experimental Interactive Learning in Industrial Management, Stockholm, Sweden, June 24-28, 2013. Revised Selected Papers. Ed. by S. A. Meijer and R. Smeds. Cham: Springer International Publishing, 2014, pp. 237-244. DOI: 10.1007/978-3-319-04954-0_28.

[82] J. Riordan. Introduction to combinatorial analysis. Courier Dover Publications, 2002 .

[83] R. J. Rossetti, J. E. Almeida, Z. Kokkinogenis, and J. Gonçalves. "Playing transportation seriously: Applications of serious games to artificial transportation systems". In: IEEE Intelligent Systems 28.4 (2013), pp. 107-112. DOI: 10.1109/MIS.2013.113.

[84] I. O. Ryzhov and W. B. Powell. "Bayesian active learning with basis functions". In: IEEE Symposium on Adaptive Dynamic Programming and Reinforcement Learning $(A D P R L)$. Winner, Best Paper, IEEE International Symposium on Adaptive Dynamic Programming and Reinforcement Learning, 2011. 2011, pp. 143-150.

[85] I. O. Ryzhov, M. R. K. Mes, W. B. Powell, and G. A. van den Berg. "Bayesian exploration for approximate dynamic programming". In: Operations Research Submitted (2017).

[86] H. P. Simao, J. Day, A. P. George, T. Gifford, J. Nienow, and W. B. Powell. "An Approximate Dynamic Programming Algorithm for Large-Scale Fleet Management: A Case Application". In: Transportation Science 43.2 (2009), pp. 178-197. DOI: $10.1287 / \operatorname{trsc} .1080 .0238$.

[87] P. Singh, M. van Sinderen, and R. Wieringa. "Synchromodal Transport: Pre-requisites, Activities and Effects". In: ILS Conference. 2016, pp. 1-4.

[88] K. Smilowitz. "Multi-resource routing with flexible tasks: an application in drayage operations". In: IIE Transactions 38.7 (2006), pp. 577-590. DOI: 10.1080 / 07408170500436898. 
[89] N. Soeffker, M. W. Ulmer, and D. C. Mattfeld. "Problem-specific state space partitioning for dynamic vehicle routing problems". In: Proceedings of MKWI (2016), pp. 229-240.

[90] M. M. Solomon. "Algorithms for the Vehicle Routing and Scheduling Problems with Time Window Constraints". In: Operations Research 35.2 (1987), pp. 254-265.

[91] J. R. Spiegel, M. T. McKenna, G. S. Lakshman, and P. G. Nordstrom. "Method and System For Anticipatory Package Shipping". English. Pat. US 8,615,473 B2. 2013.

[92] M. SteadieSeifi, N. Dellaert, W. Nuijten, T. V. Woensel, and R. Raoufi. "Multimodal freight transportation planning: A literature review". In: European Journal of Operational Research 233.1 (2014), pp. 1 -15. DOI: http://dx.doi.org/10.1016/ j.ejor.2013.06.055.

[93] J. D. Sterman. "Modeling Managerial Behavior: Misperceptions of Feedback in a Dynamic Decision Making Experiment". In: Management Science 35.3 (1989), pp. 321-339. DOI: $10.1287 / \mathrm{mnsc} .35 .3 .321$.

[94] M. Strens. "A Bayesian framework for reinforcement learning". In: ICML. 2000, pp. 943-950.

[95] T. Susi, M. Johannesson, and P. Backlund. Serious games: An overview. Tech. rep. HS- IKI -TR-07-001. University of Skvde, Sweden, 2007.

[96] R. S. Sutton and A. G. Barto. Introduction to Reinforcement Learning. 1st. Cambridge, MA, USA: MIT Press, 1998.

[97] R. S. Sutton, H. R. Maei, D. Precup, S. Bhatnagar, D. Silver, C. Szepesvári, and E. Wiewiora. "Fast Gradient-descent Methods for Temporal-difference Learning with Linear Function Approximation". In: Proceedings of the 26th Annual International Conference on Machine Learning. ICML '09. Montreal, Quebec, Canada: ACM, 2009, pp. 993-1000. DOI: 10.1145/1553374.1553501.

[98] G. Taylor, F. Broadstreet, T. S. Meinert, and J. S. Usher. "An analysis of intermodal ramp selection methods". In: Transportation Research Part E: Logistics and Transportation Review 38.2 (2002), pp. 117 -134. DOI: http://dx.doi.org/10. 1016/S1366-5545(01)00018-7.

[99] H. Topaloglu. "A parallelizable dynamic fleet management model with random travel times". In: European Journal of Operational Research 175.2 (2006), pp. 782 -805. DOI: http://doi.org/10.1016/j.ejor.2005.06.024.

[100] H. Topaloglu and W. B. Powell. "A Distributed Decision-Making Structure for Dynamic Resource Allocation Using Nonlinear Functional Approximations". In: Operations Research 53.2 (2005), pp. 281-297. DOI: 10.1287/opre.1040.0166.

[101] H. Topaloglu and W. B. Powell. "Dynamic-Programming Approximations for Stochastic Time-Staged Integer Multicommodity-Flow Problems". In: INFORMS Journal on Computing 18.1 (2006), pp. 31-42. DOI: 10.1287/ijoc.1040.0079.

[102] Topsector Logistiek. Partituur naar de top - Adviesrapport Topteam Logistiek. 2011. 
[103] Topsector Logistiek. Excel in Logistics - Top Sector Logistics. 2014. URL: http: //bit.ly/connekt-eil-uk.

[104] Topsector Logistiek. Symphony No. 2 - Results of the Top Sector Logistics. 2016.

[105] Topsector Logistiek. Uiensector Zeeland haalt 1,5 miljoen km van de weg en bespaart zon 1500 ton CO2. Dutch. 2016. URL: http://www.topsectorlogistiek.nl/ uiensector-zeeland-haalt-15-miljoen-km-van-de-weg-en-bespaart-zon1500-ton-co2/.

[106] M. W. Ulmer, L. Heilig, and S. Voß. "On the Value and Challenge of Real-Time Information in Dynamic Dispatching of Service Vehicles". In: Business $\& 3$ Information Systems Engineering 59.3 (2017), pp. 161-171. DOI: 10.1007/s12599-017-0468-2.

[107] M. W. Ulmer, D. C. Mattfeld, and F. Köster. "Budgeting Time for Dynamic Vehicle Routing with Stochastic Customer Requests". In: Transportation Science Articles in advance (2017). DOI: 10.1287/trsc.2016.0719.

[108] B. van Riessen, R. R. Negenborn, and R. Dekker. "Synchromodal Container Transportation: An Overview of Current Topics and Research Opportunities". In: Computational Logistics: 6th International Conference, ICCL 2015, Delft, The Netherlands, September 23-25, 2015, Proceedings. Ed. by F. Corman, S. Voß, and R. R. Negenborn. Lecture Notes in Computer Science. Cham: Springer International Publishing, 2015. Chap. Synchromodal Container Transportation: An Overview of Current Topics and Research Opportunities, pp. 386-397. DOI: 10.1007/978-3-319-24264-4_27.

[109] B. van Riessen, R. R. Negenborn, R. Dekker, and G. Lodewijks. "Service network design for an intermodal container network with flexible transit times and the possibility of using subcontracted transport". In: International Journal of Shipping and Transport Logistics 7.4 (2015), pp. 457-478. DOI: http://dx.doi.org/10. 1504/IJSTL . 2015.069683.

[110] M. Črepinšek, S.-H. Liu, and M. Mernik. "Exploration and Exploitation in Evolutionary Algorithms: A Survey". In: ACM Comput. Surv. 45.3 (2013), 35:1-35:33. DOI: $10.1145 / 2480741.2480752$.

[111] M. Verma and V. Verter. "A lead-time based approach for planning railtruck intermodal transportation of dangerous goods". In: European Journal of Operational Research 202.3 (2010), pp. 696 -706. DOI: http://dx.doi.org/10.1016/j.ejor. 2009.06 .005 .

[112] M. Verma, V. Verter, and N. Zufferey. "A bi-objective model for planning and managing rail-truck intermodal transportation of hazardous materials". In: Transportation Research Part E: Logistics and Transportation Review 48.1 (2012). Select Papers from the 19th International Symposium on Transportation and Traffic Theory, pp. 132 -149. DOI: http://dx.doi.org/10.1016/j.tre.2011.06.001.

[113] J. Vermorel and M. Mohri. "Multi-armed Bandit Algorithms and Empirical Evaluation". In: Machine Learning: ECML 2005: 16th European Conference on 
Machine Learning, Porto, Portugal, October 3-7, 2005. Proceedings. Ed. by J. Gama, R. Camacho, P. B. Brazdil, A. M. Jorge, and L. Torgo. Berlin, Heidelberg: Springer Berlin Heidelberg, 2005, pp. 437-448. DOI: 10.1007/11564096_42.

[114] X. Wang and A. C. Regan. "Local truckload pickup and delivery with hard time window constraints". In: Transportation Research Part B: Methodological 36.2 (2002), pp. 97 -112. DOI: http://dx.doi.org/10.1016/S0965-8564(00)00037-9.

[115] N. Wieberneit. "Service network design for freight transportation: a review". English. In: OR Spectrum 30.1 (2008), pp. 77-112. DOI: 10.1007/s00291-007-0079-2.

[116] J. Woxenius. "Generic Framework for Transport Network Designs: Applications and Treatment in Intermodal Freight Transport Literature". In: Transport Reviews 27.6 (2007), pp. 733-749. DOI: 10.1080/01441640701358796.

[117] M. Zhang and A. Pel. "Synchromodal hinterland freight transport: Model study for the port of Rotterdam". In: Journal of Transport Geography 52 (2016), pp. 1 -10. DOI: http://dx.doi.org/10.1016/j.jtrangeo.2016.02.007.

[118] H. Zolfagharinia and M. Haughton. "The benefit of advance load information for truckload carriers". In: Transportation Research Part E: Logistics and Transportation Review 70 (2014), pp. 34 -54. DOI: http://dx.doi.org/10.1016/j.tre.2014. 06.012.

[119] R. A. Zuidwijk and A. W. Veenstra. "The Value of Information in Container Transport". In: Transportation Science 49.3 (2015), pp. 675-685. DOI: 10.1287/ trsc.2014.0518. 


\section{Acronyms}

$\begin{array}{ll}\text { ADP } & \text { Approximate Dynamic Programming } \\ \text { DSND } & \text { Dynamic Service Network Design } \\ \text { FC } & \text { Fixing Criteria } \\ \text { FTPDPTW } & \text { Full Truckload Pickup and Delivery Problem with Time Windows } \\ \text { LSP } & \text { Logistic Service Provider } \\ \text { MDP } & \text { Markov Decision Process } \\ \text { MHO } & \text { Math-Heuristic Operator } \\ \text { MILP } & \text { Mixed-Integer Linear Programming } \\ \text { RQ } & \text { Research Question } \\ \text { TEU } & \text { Twenty foot Equivalent Unit } \\ \text { VFA } & \text { Value Function Approximation } \\ \text { VI } & \text { Valid Inequality } \\ \text { VPI } & \text { Value of Perfect Information } \\ \text { VRP } & \text { Vehicle Routing Problem }\end{array}$




\section{Summary}

Over the last decade, Logistic Service Providers (LSPs) have explored new approaches to control their operations with the objective of improving their network-wide efficiency and reducing their environmental impact. One of such approaches is synchromodal transport. In synchromodal transport, the choice of mode, the choice of transport path, and the timing of operations is not fixed up front, but decided upon various moments using the latest information about the status of the multi-modal network and about freight demand. This increased flexibility provides LSPs with more opportunities for consolidation and options for efficient transport, throughout the network and throughout time. However, to achieve such gains, transport decisions must consider their consequences in the entire network and anticipate on their effect in future decisions and performance. In this dissertation, we study how to make such decisions in synchromodal transport and what improvement opportunities can be expected.

Our work deals with anticipatory scheduling decisions for the transport of freight in a synchromodal network. These decisions are dynamic, i.e., change when new information is revealed, and consider current and future freight demand and performance. We use four different perspectives for decisions on a multi-modal network, based on traditional scheduling methods considered in literature. For each perspective, we develop mathematical models and heuristic algorithms that support anticipatory scheduling decisions. Furthermore, for each perspective, we evaluate the output of our models and algorithms using simulation-based experiments, and provide insights into their efficiency gains, over traditional scheduling methods, using different network characteristics. These perspectives are as follows.

First perspective: We study the scheduling of long-haul transport in a network without transfers (e.g., train running in a corridor, barge traveling between the hinterland and a deep-sea port). We model the problem as a Markov Decision Process (MDP) and design an Approximate Dynamic Programming (ADP) algorithm to solve the model heuristically. We design different ADP architectures and provide insights into the design and evaluation challenges of using ADP in our problem. We demonstrate the effect of various network characteristics on the cost savings of using our approach 
instead of single-period optimization. The savings range between $6 \%$ and $9 \%$, on average in 7 out of the 8 instance categories tested, and up to $26 \%$ in some cases. Our approach performs best with unbalanced demand and a majority of pre-announced orders.

Second perspective: We study the scheduling of long-haul transport in a network with intermediate terminals and transfers. We extend the MDP model of the first perspective and use mathematical programming techniques to capture the space-time evolution of freights in the network. We extend the ADP algorithm with additional components from Bayesian exploration, specifically the Value of Perfect Information (VPI), to handle the complex relation between performance over time and the space-time evolution of freights in the network. We describe how the one-step look-ahead perspective of traditional ADP can make the algorithm flounder and end in a local-optimum, and how through the quantification of the value of exploration in VPI, the ADP algorithm can escape this local-optimum and improve the solution. We show how our proposed ADP-VPI combination, with a heavy restriction on the decision space, achieves more than $20 \%$ gains over the profit achieved by common practice unrestricted heuristics. When considering the decision space restrictions as part of the problem, the gains are larger than $50 \%$ in 6 out of the 9 instance categories tested, and around $200 \%$ in 3 of these 6 . The distribution of time-windows was the key characteristic influencing the gains of our approach in our experiments.

Third perspective: We study the scheduling of drayage operations considering initial terminal flexibility and a categorization of jobs. Drayage operations, also known as pre- and end-haulage, are a special case of the vehicle routing problem where timing, routing, and long-haul terminal assignment decisions are integrated and simultaneously considered. We develop a Mixed-Integer Linear Programming (MILP) model of the problem and design a series of valid inequalities and pre-processing mechanisms to reduce its complexity. We design two matheuristics to solve the MILP model in a static and dynamic way. These matheuristics iteratively confine the solution space of the MILP using several adaptations, and based on the incumbent solutions, guide the subsequent iterations and solutions. We benchmark our approach against a heuristic from literature and observe cost savings between $3 \%$ and $4 \%$ in 3 out of the 4 instance categories tested. Our approach performs best with clustered locations and short timewindows.

Fourth perspective: We study the integrated scheduling of drayage and long-haul transport. We combine the models and heuristics from the second and third perspective, and design an iterative simulation approach that outputs a unified decision policy (i.e., function for anticipatory scheduling that considers the current status of the network and future performance) for scheduling drayage operations and long-haul transport. The approach from the second perspective yields esti- 
mates of future long-haul costs, which are used to assign terminals to incoming pre-haulage freights in the approach of the third perspective. We show that our approach results in cost savings between $4 \%$ and $38 \%$ in all instance categories tested. Our methods achieves the largest savings with a balanced destination distribution.

As an addition to the scheduling methods, we study how to increase awareness about the trade-offs considered by our methods and how to facilitate the adoption of the algorithms from the first perspective through a serious game. We develop a web-based game that educates on the benefits of anticipatory decisions and demonstrates how our algorithms can support an LSP planner. We verify and validate our game design in gaming sessions with students of Industrial Engineering and Management at the University of Twente.

Finally, we present a closing reflection about our anticipatory scheduling methods for freights in synchromodal transport, and provide an outlook for further research with respect to extensions of the models, improvements of the heuristics, and implementation aspects. 
212 


\section{Samenvatting}

In het afgelopen decennium hebben logistieke dienstverleners (LSP's) nieuwe benaderingen onderzocht om hun activiteiten te beheersen met als doel de netwerkbrede efficiëntie te verbeteren en de duurzaamheid te vergroten. Eén van deze benaderingen is synchromodaal transport. Bij synchromodaal transport is de keuze van de transportmodus, de keuze van het transportpad en de timing van bewerkingen niet vooraf vastgelegd, maar wordt het op verschillende momenten besloten met behulp van de meest recente informatie over de status van het multimodale netwerk en de transportbehoefte. Deze verhoogde flexibiliteit biedt LSP's meer mogelijkheden voor consolidatie en opties voor efficiënt transport, over het gehele netwerk en over verloop van tijd. Om dergelijke voordelen te behalen, moeten transportbeslissingen echter de gevolgen ervan in het hele netwerk in overweging nemen en anticiperen op hun effect op toekomstige beslissingen en prestaties. In dit proefschrift bestuderen we hoe we dergelijke beslissingen kunnen nemen in synchromodaal transport en welke verbeteringen te verwachten zijn.

Ons werk richt zich op anticiperende planningsbeslissingen voor het transport van vracht in een synchromodaal netwerk. Deze beslissingen zijn dynamisch, d.w.z. veranderen wanneer nieuwe informatie bekend wordt en houden rekening met de huidige en toekomstige transportvraag en -prestaties. We gebruiken vier verschillende perspectieven voor beslissingen in een multimodaal netwerk, gebaseerd op traditionele planningsmethoden die in de literatuur worden beschouwd. Voor elk perspectief ontwikkelen we wiskundige modellen en heuristische algoritmen die anticiperende planningsbeslissingen ondersteunen. Vervolgens evalueren we voor elk perspectief de output van onze modellen en algoritmen met behulp van op simulatie gebaseerde experimenten en geven inzicht in hun efficiëntiewinsten ten opzicht van traditionele planningsmethoden, gebruikmakend van verschillende netwerkkarakteristieken. Deze perspectieven zijn als volgt.

Eerste perspectief: We bestuderen de planning van langeafstandsvervoer in een netwerk zonder transfers (bijvoorbeeld een treintraject of de binnenvaartschip tusseen een zeehaven en het achterland). We modelleren het probleem als een Markov-beslissingsproces (MDP) en stellen een Approximate Dynamic Programming (ADP) -algoritme op om het model heuristisch op te lossen. We ontwerpen verschillende ADP-architecturen en 
bieden inzicht in de ontwerp- en evaluatie-uitdagingen van het gebruik van ADP in ons probleem. We demonstreren het effect van verschillende netwerkkarakteristieken op de kostenbesparingen van het gebruik van onze aanpak in vergelijking met een korte termijn optimalisatiemethode. De besparingen liggen tussen $6 \%$ en $9 \%$, gemiddeld in 7 van de 8 geteste subcategorieën, en in sommige gevallen tot 26\%. Onze aanpak presteerd het best in een situatie met een onevenwichtige vraag en een meerderheid van vooraf aangekondigde orders.

Tweede perspectief: We bestuderen de planning van langeafstandsvervoer in een netwerk met tussenliggende terminals en transfers. We breiden het MDP-model van het eerste perspectief uit en gebruiken wiskundige programmeertechnieken om de ruimte-tijdevolutie van vrachten in het netwerk vast te leggen. We breiden het ADP-algoritme uit met extra componenten uit de Bayesiaanse exploratie, met name de waarde van perfecte informatie (VPI), om de complexe relatie tussen prestaties in de tijd en de ruimte-tijdevolutie van vrachten in het netwerk te modelleren. We beschrijven hoe het korte termijn perspectief van traditionele ADP algoritmen onvoldoende exploreren en daarmee in een lokaal optimum kunnen belanden, en hoe door de kwantificatie van de waarde van informatie in VPI, het ADP-algoritme in staat stelt te ontsnappen aan dit lokaal-optimum en de oplossing te verbeteren. We laten zien hoe onze voorgestelde ADP-VPI-combinatie, met een grote beperking op de beslissingsruimte, meer dan $20 \%$ winst behaalt ten opzichte van de winst die wordt behaald door algemene in de praktijk gebruikte restrictievrije heuristieken. Wanneer de beperkingen deel zijn van het probleem, zijn de winsten groter dan $50 \%$ in 6 van de 9 geteste subcategorieën, en ongeveer $200 \%$ in 3 van deze 6 . De verdeling van tijdvensters heeft de grootste invloed op de winst van onze aanpak.

Derde perspectief: We bestuderen de planning van drayage-operaties, rekening houdend met de initiële terminalflexibiliteit en een categorisering van werkzaamheden. Drayageoperaties, ook bekend als voor- en eind-transport, zijn een speciaal geval van het voertuigrouteringsprobleem, waarbij beslissingen over timing, routering en lange-termijn terminaltoewijzing geïntegreerd zijn en gelijktijdig worden overwogen. We ontwikkelen een Mixed-Integer Lineair Programmering (MILP) model van het probleem en ontwerpen een reeks valid inequalities en pre-processing mechanisms om de complexiteit ervan te verminderen. We ontwerpen twee matheuristics om het MILP-model op een statische en dynamische manier op te lossen. Deze matheuristieken beperken iteratief de oplossingsruimte van de MILP met behulp van verschillende aanpassingen en sturen op basis van de bestaande oplossingen de daaropvolgende iteraties en oplossingen. We benchmarken onze benadering tegen een heuristiek uit de literatuur en zien kostenbesparingen tussen de $3 \%$ en $4 \%$ in 3 van de 4 geteste subcategorieën. Onze aanpak presteerd het best met geclusterde locaties en korte tijdvensters. 
Vierde perspectief: We bestuderen de geïntegreerde planning van drayage en langeafstandsvervoer. We combineren de modellen en heuristieken vanuit het tweede en derde perspectief en ontwerpen een iteratieve simulatie-aanpak die bestaande uit een uniform beslissingsbeleid (d.w.z. functie voor anticiperende planning die rekening houdt met de huidige status van het netwerk en toekomstige prestaties) voor het plannen van drayage-operaties en lange-afstandtransport. De benadering vanuit het tweede perspectief levert schattingen op van toekomstige langeafstandskosten, die worden gebruikt om terminals toe te wijzen aan binnenkomende pre-transport-vrachten in de methode van het derde perspectief. We laten zien dat onze aanpak resulteert in kostenbesparingen van $4 \%$ tot $38 \%$ in alle geteste subcategorieën. Onze methoden behalen de grootste besparingen met een gebalanceerde bestemmingsverdeling.

Als aanvulling op de planningsmethoden, bestuderen we hoe we het inzicht kunnen vergroten van de afwegingsprocessen van onze methoden en hoe we de adoptie van de algoritmen vanuit het eerste perspectief met behulp van een computer spel (serious game) kunnen vergemakkelijken. We ontwikkelen een online spel dat inzicht geeft in de voordelen van anticiperende beslissingen en laat zien hoe onze algoritmen een LSP-planner kunnen ondersteunen. We verifiëren en valideren ons spelontwerp in spelsessies met studenten van de Masteropleiding Industrial Engineering and Management aan de Universiteit Twente.

Ten slotte reflecteren we op onze anticiperende planningsmethoden voor vrachten in synchromodaal transport en geven we een vooruitblik voor verder onderzoek met betrekking tot uitbreidingen van de modellen, verbeteringen van de heuristieken en implementatieaspecten. 


\section{Resumen}

Durante la última década, los proveedores de servicios logísticos (LSP por sus siglas en Inglés) han explorado nuevos enfoques para controlar sus operaciones con el objetivo de mejorar la eficiencia de su red de transporte y reducir su impacto ambiental. Uno de esos enfoques es el transporte sincromodal. En el transporte sincromodal, la elección del modo de transporte, la elección de la ruta de transporte y el inicio de las operaciones no se fijan por adelantado, sino que se deciden en varios momentos del proceso utilizando la última información sobre el estado de la red de transporte multimodal y sobre la demanda de transporte. Esta mayor flexibilidad proporciona a los LSP más oportunidades de consolidación y más opciones para un transporte eficiente, a lo largo de la red y en el transcurso del tiempo. Sin embargo, para lograr realizar esas oportunidades y obtener tales mejoras, las decisiones de transporte deben considerar sus consecuencias en toda la red y prever su efecto en las decisiones y el desempeño futuros. En esta tesis, estudiamos cómo tomar tales decisiones en el transporte sincromodal y qué oportunidades de mejora se pueden esperar.

Nuestro trabajo se ocupa de las decisiones de programación anticipatoria para el transporte de carga en una red sincromodal. Estas decisiones son dinámicas, es decir, cambian cuando se revela información nueva y consideran la demanda y el rendimiento de los transportes actuales y futuros. Para estudiar estas decisions, utilizamos cuatro perspectivas diferentes en una red de transporte multimodal basadas en los métodos tradicionales de programación considerados en la literatura científica. Para cada perspectiva, desarrollamos modelos matemáticos y algoritmos heurísticos que respaldan las decisiones de programación anticipatoria. Además, para cada perspectiva, evaluamos el rendimiento de nuestros modelos y algoritmos utilizando experimentos basados en simulación y brindamos información sobre sus mejoras de eficiencia, en comparación a los métodos tradicionales de programación y usando diferentes características de red de transporte. Estas perspectivas son las siguientes.

Primera perspectiva: Estudiamos la programación del transporte de larga distancia en una red multimodal sin transferencias (por ejemplo, un tren en un corredor logístico o una barcaza viajando del interior del territorio a un puerto de alta mar). Modelamos 
el problema como un Proceso de Decisión de Markov (MDP por sus siglas en Inglés) y diseñamos un algoritmo de Programación Dinámica Aproximada (ADP por sus siglas en Inglés) para resolver el modelo heurísticamente. Diseñamos diferentes arquitecturas de ADP y proporcionamos información sobre los desafíos de diseño y evaluación por el uso de ADP en nuestro problema. Demostramos el efecto de varias características de la red en el ahorro de costos al utilizar nuestro enfoque en lugar de la optimización de corto plazo. El rango de ahorro oscila entre el $6 \%$ y el $9 \%$, en promedio en 7 de las 8 categorías de instancias probadas, y hasta el $26 \%$ en algunos casos. Nuestro enfoque se desempeñó mejor con la demanda desequilibrada y la mayoría de los pedidos anunciados previamente.

Segunda perspectiva: Estudiamos la programación del transporte de larga distancia en una red multimodal con terminales y transferencias intermedias. Extendemos el modelo MDP de la primera perspectiva y usamos técnicas de programación matemática para capturar la evolución del espacio-tiempo de los transportes en la red. Extendemos el algoritmo ADP con componentes adicionales de la exploración Bayesiana, específicamente el Valor de la Información Perfecta (VPI por sus siglas en Ingles), para controlar la relación compleja entre el rendimiento en el tiempo y la evolución del espacio-tiempo de las cargas en la red. Describimos cómo la perspectiva de un paso adelante (o etapa siguiente del horizonte) del ADP tradicional puede hacer que el algoritmo falle y termine en un óptimo local, y cómo a través de la cuantificación de un valor de exploración en VPI, el algoritmo ADP puede escapar de este óptimo local y mejorar la solución. Mostramos cómo nuestra combinación propuesta de ADP-VPI, con una gran restricción en el espacio de decisión, logra más del $20 \%$ de ganancias sobre el beneficio logrado por la práctica común de program el transporte con una heurística no restringida. Al considerar las restricciones de espacio de decisión como parte del problema, las ganancias son mayores al $50 \%$ en 6 de las 9 categorías de instancia probadas, y alrededor de $200 \%$ en 3 de estas 6 . La distribución de las ventanas (o restricciones) de tiempo fue la característica clave que influyó en las ganancias de nuestro enfoque.

Tercera perspectiva: Estudiamos la programación de las operaciones de traslado corto teniendo en cuenta la flexibilidad al escoger una terminal inicial y una categorización de órdenes de transporte. Las operaciones de traslado corto, también conocidas como acarreo previo y final, son un caso especial del problema de enrutamiento de vehículos donde las decisiones de asignación de tiempo, enrutamiento y de asignación de terminal inicial se integran y se consideran simultáneamente. Desarrollamos un modelo de problema usando Programación Lineal Entera Mixta (MILP por sus siglas en Inglés) y diseñamos una serie de desigualdades válidas y mecanismos de pre-procesamiento para reducir su complejidad. Diseñamos dos algoritmos de heurísticas matemáticas para resolver el modelo de MILP de una manera estática y dinámica. Estos algoritmos confinan iterativamente el espacio de la solución del MILP usando varias adaptaciones, y basados en las soluciones predominantes, dirigen las iteraciones y las soluciones subsecuentes. 
Comparamos nuestro enfoque con una heurística de la literatura científica y observamos ahorros de costos entre $3 \%$ y $4 \%$ en 3 de las 4 categorías de instancia probadas. Nuestro enfoque se desempeñó mejor con ubicaciones agrupadas y ventanas de tiempo cortas.

Cuarta perspectiva: Estudiamos la programación integrada de transporte de larga distancia y acarreo previo y final. Combinamos los modelos y la heurísticas desarrolladas en la segunda y tercera perspectiva, y diseñamos un enfoque iterativo de simulación que emite una política de decisión unificada. Esta política es una función para la programación anticipatoria que considera el estado actual de la red y el rendimiento futuro para programar operaciones de acarreo y transporte de larga distancia. El enfoque de la segunda perspectiva arroja estimaciones de los costos futuros de larga distancia, que se utilizan para asignar terminales iniciales a las cargas entrantes de acarreo previo en el enfoque de la tercera perspectiva. Mostramos que nuestro enfoque resulta en ahorros de costos entre $4 \%$ y $38 \%$ en todas las categorías de instancia probadas. Nuestros métodos lograron los mayores ahorros con una distribución de destino equilibrada.

Adicionalmente a los métodos de programación anticipatoria, estudiamos cómo aumentar la conciencia sobre las concesiones consideradas por nuestros métodos y cómo facilitar la adopción de los algoritmos de la primera perspectiva a través de un juego serio o juego educativo. Desarrollamos un juego basado en el Internet que educa sobre los beneficios de las decisiones anticipatorias y demuestra cómo nuestros algoritmos pueden apoyar a un planificador de LSP. Verificamos y validamos nuestro diseño del juego en sesiones de juego con estudiantes de Ingeniería y Gestión Industrial en la Universidad de Twente.

Finalmente, presentamos una reflexión final sobre nuestros métodos de programación anticipatoria para las cargas en el transporte sincromodal, y brindamos una perspectiva para futuras investigaciones científicas relacionadas con extensiones de los modelos, mejoras de las heurísticas y aspectos de implementación. 


\section{Scientific Output}

\section{Publications}

A.E. Pérez Rivera, M.R.K. Mes, J. van Hillegersberg (2018). A Simulation Game for Anticipatory Scheduling of Synchromodal Transport. Full paper. ISAGA 2018 Conference Proceedings, Nakhorn Pathom, Thailand.

A.E. Pérez Rivera, M.R.K. Mes (2018). Integrated Scheduling of Drayage and Long-haul Transportation in Synchromodality. Extended Abstract. Odysseus 2018 Conference Proceedings, Cagliari, Italy.

A.E. Pérez Rivera, M.R.K. Mes (2018). Integrated scheduling of drayage and long-haul operations in synchromodal transport. Working Paper.

A.E. Pérez Rivera, M.R.K. Mes (2017). Anticipatory Scheduling of Freight in a Synchromodal Transportation Network. Beta Working Paper 533.

A.E. Pérez Rivera, M.R.K. Mes (2017). Scheduling Drayage Operations in Synchromodal Transport. In Bektas, T., et al. (Eds.), Computational Logistics - ICCL 2017, Lecture Notes in Computer Science, Volume 10572, pp. 404-419. Springer. DOI 10.1007/978-3-319-68496$3 \_27$

M.R.K. Mes, A.E. Pérez Rivera (2017). Approximate Dynamic Programming by Practical Examples. In: Richard Boucherie and Nico M. van Dijk (Eds.), Markov Decision Processes in Practice. International Series in Operations Research and Management Science, Volume 248, pp. 63-101. Springer. DOI 10.1007/978-3-319-47766-4_3

A.E. Pérez Rivera, M.R.K. Mes (2016). Service and transfer selection for freights in a synchromodal network. In Paias, A., Ruthmair, M., and Voß, S. (Eds.), Computational Logistics - ICCL 2016, Lecture Notes in Computer Science, Volume 9855, pp. 227-242. Springer. DOI 10.1007/978-3-319-44896-1_15 
A.E. Pérez Rivera, M.R.K. Mes (2016). Anticipatory Freight Selection in Intermodal Longhaul Round-trips. Transportation Research Part E: Logistics and Transportation Review. Volume 105, pp. 176-194, 2017, Elsevier. DOI 10.1016/j.tre.2016.09.002

A.E. Pérez Rivera, M.R.K. Mes (2016). Pre- and end-haulage operations in a multi-depot and multi-resource synchromodal network. Extended Abstract. TRISTAN 2016 Conference Proceedings, Oranjestad, Aruba.

A.E. Pérez Rivera, M.R.K. Mes (2015). Dynamic Multi-period Freight Consolidation. In Corman, F., Voß, S., and Negenborn, R.R. (Eds.), Computational Logistics - ICCL 2015, Lecture Notes in Computer Science, Volume 9335, pp. 370-385. Springer. DOI 10.1007/9783-319-24264-4_26

M.R.K. Mes, J.M.J. Schutten, A.E. Pérez Rivera (2014). Inventory routing for dynamic waste collection. Waste Management, Volume 34, Issue 9, pp. 1564-1576, 2014, Elsevier. DOI 10.1016/j.wasman.2014.05.011

\section{Conference and Seminar Presentations}

A.E. Pérez Rivera (2018). Anticipatory Freight Scheduling in Synchromodal Transport. Research Seminar at the Technical University Braunschweig. Presented on 19/06/2018, Braunschweig, Germany.

A.E. Pérez Rivera (2018). Integrated Scheduling of Drayage and Long-haul Transportation in Synchromodality. Triennial International Workshop on Freight Transportation and Logistics (Odysseus). Presented on 07/06/2018, Cagliari, Italy.

A.E. Pérez Rivera (2018). Combined Scheduling of Pre-haulage and Long-haul Freight Transportation. Escuela Latinoamericana de Verano en Investigación Operativa (ELAVIO). Presented on 05/03/2018, Maintencillo, Chile.

A.E. Pérez Rivera (2017). Scheduling Drayage Operations in Synchromodal Transport. International Conference on Computational Logistics (ICCL). Presented on 19/10/2017, Southampton, United Kingdom.

A.E. Pérez Rivera (2017). Anticipatory scheduling of freight in a synchromodal transport network. . 14th Beta PhD symposium. Presented on 26/09/2017, Eindhoven, The Netherlands.

A.E. Pérez Rivera (2017). Integrated scheduling in synchromodal transport. International Conference on Logistics and Maritime Systems (LOGMS). Presented on 24/08/2017, Bergen, Norway. 
A.E. Pérez Rivera (2017). Scheduling synchromodal freight transport using approximate dynamic programming. Annual meeting of the EURO Working Group on Vehicle Routing and Logistics Optimization (VeRoLog). Presented on 12/07/2017, Amsterdam, The Netherlands.

A.E. Pérez Rivera (2016). Service and transfer selection for freights in a synchromodal network. International Conference on Computational Logistics (ICCL). Presented on 09/09/2016, Lisbon, Portugal.

A.E. Pérez Rivera (2016). Pre- and end-haulage operations in a multi-depot and multi-resource synchromodal network. Triennial Symposium on Transportation Analysis (TRISTAN). Presented on 17/06/2016, Oranjestad, Aruba.

A.E. Pérez Rivera (2016). Anticipatory freight consolidation in intermodal networks using approximate dynamic programming. Research Seminar at the Erasmus Research Institute of Management. Presented on 20/05/2016, Rotterdam The Netherlands.

A.E. Pérez Rivera (2015). Dynamic multi-period freight consolidation. International Conference on Computational Logistics (ICCL). Presented on 24/09/2015, Delft, The Netherlands.

A.E. Pérez Rivera (2015). Dynamic freight selection for reducing long-haul round trip costs. Annual meeting of the EURO Working Group on Vehicle Routing and Logistics Optimization (VeRoLog). Presented on 10/06/2015, Vienna, Austria.

A.E. Pérez Rivera (2014). Long-haul freight selection for last-mile cost reduction. Annual meeting of the Institute for Operations Research and the Management Sciences (INFORMS). Presented on 11/11/2014, San Francisco, CA, USA. 


\section{Acknowledgements / Agradecimientos}

Preparing my doctoral dissertation was an enriching experience and a wonderful adventure. During this time, I had the support, guidance, friendship, and inspiration of many people. In the following paragraphs, I acknowledge these colleagues, friends, and family who contributed, in their own way, to the culmination of my Ph.D.

I am thankful to my promotor, Prof. van Hillegersberg, for his support and supervision during my time as a doctoral candidate. Jos, your advice helped me writing this thesis and delving into serious games. In addition, your endorsement of my work initiatives and extra-curricular activities made my Ph.D. time more gratifying. Thank you for the opportunity of doing my doctoral work in the IEBIS department.

I am profoundly grateful to my co-promotor, Dr. ir. Mes, for his guidance and inspiration before, during, and after my time as a doctoral candidate. Martijn, your continuous support and valuable advice have been crucial for my academic accomplishments. Your keen mind for mathematical models, effective judgment of algorithmic challenges, and constructive criticism of writing style significantly helped me in my development as a scientist. Furthermore, your people skills inspired and motivated me immensely. Our long and constructive discussions were encouraging, especially the spontaneous ones with a jug of coffee. Our conference trips, national travels, and home-cooked dinners and rummikub games with Edith, Sophie, and Olivier, were always fun and strengthened the team spirit. In short, thank you for your scientific advices and personal leadership. I could not have imagined having a better advisor and mentor for my Ph.D.

My gratitude also goes to the rest of my graduation committee, Prof. Boucherie, Prof. Iacob, Prof. Mattfeld, Prof. Tavasszy, Prof. Voß, Prof. Zijm, and Prof. Zuidwijk for studying my doctoral dissertation. I am honored to defend my thesis against such eminent opponents and discuss my research with such knowledgeable scientists. 
Many thanks to the staff at the IEBIS department for the inspiring discussions and collaborations around the workplace. Particularly, I thank my fellow doctoral candidates and post-doctoral researchers for the nice moments around the Ravelijn building and around Enschede. I am grateful to my office mates Arviansyah, Engin, Fabian, Nils, Rick, Sajjad, Sina, and Xavier, for the coffee ceremonies, interesting debates, and good memories. You guys always made of our office a cheerful place to be at. Wouter, thank you for all the elucidating conversations about work and about life, as well as the great times we had traveling or having a drink. Thanks also to Abhishta, Andrej, Devrim, Dissa, Gréanne, Guido, Luca, Miha, Sjoerd, Rudy, Vahid, and Wenyi for the coffee breaks, lunch walks, barbecues and outings in Enschede.

My gratitude also for the friends that I made during my extra-curricular activities and volunteer work in Enschede. Andrea, Didre, and Naveen, thank you for the rewarding work we did for UniTe. Your motivation to improve the international student-life was contagious. Aryo, Claudio, Jennifer, Paola, Ricardo, Uun, Yolla and all the Ariëns Catholic Students, thank you for the times we came together to keep the flame of faith in God alive. Your spirit of service was always inspiring.

I express my appreciation to all my friends around the globe, who have always been close despite distance and time. To the friends I met in Enschede during my time as a doctoral candidate, Armando, Björn, Emad, Enrique, Ernesto, Gisela, Ioana, Jasmin, Laura, Vanessa, and Wessel, thank you for the enjoyable conversations and the numerous moments we shared dinner and drinks. These fun moments gave me much happiness and relieved the mental pressure of scientific research. To the friends I met during my time in Amsterdam, Alessandra, Chelsea, Ewald, Ines, Marlies, Núria, and Peter, thank you for the pleasant visits/hosting and for the memorable times together. Meeting you all around Europe was always delightful. To the friends I met during my time as a master student in Enschede, Daniel, Harm, Kelvin, Lara, and Moon Hee, thank you for keeping in touch after all these years. Meeting you in different parts of The Netherlands was always energizing and enjoyable.

An enormous gratitude goes to my paranymphs: Rick van Urk and Javier A. Morán M., who both supported me from the beginning to the end of my Ph.D. work. Rick, hartelijke dank voor je geweldige vriendschap. Je vrolijke houding heeft me altijd gemotiveerd en je permanente beschikbaarheid is iets waar ik altijd op heb vertrouwd. Javier, estimadísimo, muchas gracias por tu amistad fraternal. Aprecio tu iniciativa constante y tus atenciones amables conmigo y con mi familia. Thank you guys also for the (international) trips, tasty dinners, and great moments during my Ph.D.!

Agradezco a mis amigos Mexicanos (C-r.e.H.): Alejandro, Ana, Arturo, Carol, Daniella, Diana, Edgar, Héctor, Imke, Javier, Martín, Mathijs, Sander y Simone por su muy bonita amistad. Durante los últimos años, hemos compartido numerosas veces y hemos celebrado, 
y seguimos celebrando, muchas ocasiones especiales. Su amabilidad, afecto y buen sentido del humor hacen de cada momento que estamos juntos un tiempo especial. Llevo en mi memoria esas carnes asadas, roscas de reyes, taquitos, tequilas, juegos, canciones, viajes, etc. que hicieron de las exigencias del mundo académico más llevaderas. ¡Mi agradecimiento por su amistad y mi deseo de seguirla cultivando!

Muchísimas gracias / muito obrigado a mis amigos de la asociación de estudiantes latinoamericanos en la Universidad de Twente, LA Voz: Agustín, Bruno, Ivette, Peter, Rana, Reynaldo, Rudolph, Sergio, Valentina y Vania. Durante mi tiempo en la junta directiva, disfruté mucho trabajando con ustedes, compartiendo nuestra cultura con el resto del mundo y haciendo sentir en casa a nuestros coterráneos. Pero el resultado más gratificante de esa experiencia fue la maravillosa amistad de ustedes. Su carácter simpático, sus ocurrencias y su compañía agradable fueron, y son, motivo de mucha alegría. Recuerdo las cenas y karaokes caseros, los intercambios navideños y los tantos momentos gratos que compartimos. ¡Qué sigan sucediendo!

Estoy también muy agradecido con mis amigos Hondureños, a los que año tras año he compartido en época de Navidad: Allan, Christian, Elio, Fernando, Héctor, Hugo, Juan José, Mauro Z. y Omar. A pesar de la distancia y del tiempo, siempre he podido contar con su amistad y su estima. Valoro mucho nuestras reuniones y, más aún, nuestras conversaciones sobre casi todos los temas imaginables (con unas 2Q3). Les guardo un gran aprecio y espero la vida nos siga regalando muchísimas oportunidades para compartir, aquí, allá, y en cualquier parte del mundo.

Agradezco a toda mi familia que me ha apoyado y dado su cariño desde siempre. Agradezco a mis tías(os) que pasan pendientes de mí y que me llenan de sus atenciones y palabras amables cada vez que nos reunimos, especialmente a mis tías Delmy, Elvia, Nelly A., Nelly P., Nora y Olga. Agradezco a mis primas(os) con quienes he compartido buenos momentos, especialmente a Benjamín J., Carlos X., Cynthia, David, Denisse, Eduardo, Héctor Román, Iván, Katherine, Kelly, Laura M., Salvador, Samuel, Sonia A., Sonia G. y Wilfredo. Agradezco a Juanita, por sus detalles cariñosos conmigo y con mi padres. A todos ustedes, les llevo en mi mente y en mi corazón.

Y por último, agradezco inmensamente a mis padres, Roselia Arelí y Justo Edelmín, por sus sabios consejos, su inagotable comprensión, sus inolvidables visitas y su cariño sin igual. A ellos dedico este libro. Mamá, tu dedicación al trabajo y tu responsabilidad, me han servido de ejemplo para alcanzar mis metas. Papá, tu sabiduría y tu confianza me han inculcado a ser mejor cada día. Les doy gracias por todo lo que incondicionalmente me han dado y me sigue dando. ¡Qué este logro de alcanzar mi doctorado nos brinde nuevas oportunidades!

Arturo E. Pérez Rivera, Enschede, $28^{\text {th }}$ of May 2018. 


\section{About the Author}

Arturo E. Pérez Rivera was born on the 2nd of March of 1988, in San Pedro Sula, Honduras. In 2005, he finished secondary school in Freedom High School, in San Pedro Sula, and was chosen Valedictorian among his graudating class. In 2009, he obtained his bachelor's degree in Industrial and Systems Engineering from the Universidad Tecnolgica Centroamericana in San Pedro Sula, Honduras, graduated with highest honors (summa cum laude) and was chosen Valedictorian among his graduating class. From 2009 to 2010, he worked as Samples Manager of Garan Buenavista, a children's garments production plant in Villanueva, Honduras. In 2012, he obtained his master's degree in Industrial Engineering and Management from the University of Twente, in Enschede, The Netherlands. From 2012 to 2014, he worked as Logistics Analyst in the Antoni van Leeuwenhoek Hospital, a cancer-specialized center in Amsterdam, The Netherlands.

In 2014, Arturo started as a doctoral candidate within the Department of Industrial Engineering and Business Information Systems at the University of Twente, on the DINALOG project SynchromodalIT. During his time as a doctoral candidate, Arturo was an active volunteer in the international community of the University of Twente and Enschede. At the University of Twente, he was President (2015-2016) and Treasurer (2014-2015) of the Latin American Student Association LA VOZ, interim President (2017) and member of the Steering Committee (since 2017) of UniTe, the umbrella association for World Associations of the Student Union, and Twente Representative (2015-2017) in the Beta PhD Council of the Beta Research School. In Enschede, he is part of the Pastoral management group and co-organizer of the International Mass (since 2015) at the St. James Roman Catholic church. 